\title{
The HITRAN2016 molecular spectroscopic database
}

\author{
I. E. Gordon \\ IGordon@cfa.harvard.edu \\ L. S. Rothman \\ C. Hill \\ R. V. Kochanov \\ Y. Tan
}

See next page for additional authors

Follow this and additional works at: https://scholarworks.wm.edu/aspubs

\section{Recommended Citation}

Gordon, I. E.; Rothman, L. S.; Hill, C.; Kochanov, R. V.; Tan, Y.; Bernath, P. F.; Birk, M.; Boudon, V.; Campargue, A.; Chance, K. V.; Drouin, B. J.; Flaud, J. -M.; Gamache, R. R.; Hodges, J. T.; Jacquemart, D.; Perevalov, V. I.; Perrin, A.; Shine, K. P.; Smith, M. -A. H.; Tennyson, J.; Toon, G. C.; Tran, H.; Tyuterev, V. G.; Barbe, A.; and Devi, V. Malathy, The HITRAN2016 molecular spectroscopic database (2017). JOURNAL OF QUANTITATIVE SPECTROSCOPY \& RADIATIVE TRANSFER, 203.

10.1016/j.jqsrt.2017.06.038

This Article is brought to you for free and open access by the Arts and Sciences at W\&M ScholarWorks. It has been accepted for inclusion in Arts \& Sciences Articles by an authorized administrator of W\&M ScholarWorks. For more information, please contact scholarworks@wm.edu. 


\section{Authors}

I. E. Gordon, L. S. Rothman, C. Hill, R. V. Kochanov, Y. Tan, P. F. Bernath, M. Birk, V. Boudon, A. Campargue, K. V. Chance, B. J. Drouin, J. -M. Flaud, R. R. Gamache, J. T. Hodges, D. Jacquemart, V. I. Perevalov, A.

Perrin, K. P. Shine, M. -A. H. Smith, J. Tennyson, G. C. Toon, H. Tran, V. G. Tyuterev, A. Barbe, and V. Malathy Devi 


\section{Journal of Quantitative Spectroscopy \& Radiative Transfer}

journal homepage: www.elsevier.com/locate/jqsit

\section{The HITRAN2016 molecular spectroscopic database}

I.E. Gordon ${ }^{\mathrm{a}, *}$, L.S. Rothman ${ }^{\mathrm{a}}$, C. Hill ${ }^{\mathrm{a}, \mathrm{b}}$, R.V. Kochanov ${ }^{\mathrm{a}, \mathrm{c}}$, Y. Tan ${ }^{\mathrm{a}}$, P.F. Bernath ${ }^{\mathrm{d}}$, M. Birk ${ }^{\mathrm{e}}$, V. Boudon ${ }^{f}$, A. Campargue ${ }^{g}$, K.V. Chance ${ }^{a}$, B.J. Drouin ${ }^{\text {h, J.-M. Flaud }}{ }^{i}$, R.R. Gamache ${ }^{j}$, J.T. Hodges $^{k}$, D. Jacquemart ${ }^{1}$, V.I. Perevalov ${ }^{m}$, A. Perrin ${ }^{n}$, K.P. Shine ${ }^{0}$, M.-A.H. Smith ${ }^{p}$,

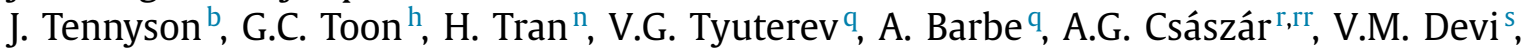
T. Furtenbacher ${ }^{r}$, J.J. Harrison ${ }^{\text {t,tt,ttt }}$, J.-M. Hartmann ${ }^{\mathrm{n}}$, A. Jolly ${ }^{\mathrm{i}}$, T.J. Johnson ${ }^{\mathrm{u}}$, T. Karman ${ }^{\mathrm{a}, \mathrm{v}}$, I. Kleiner ${ }^{\mathrm{i}}$, A.A. Kyuberis ${ }^{\mathrm{a}, \mathrm{w}}$, J. Loos ${ }^{\mathrm{e}}$, O.M. Lyulin ${ }^{\mathrm{m}}$, S.T. Massie ${ }^{\mathrm{x}}$, S.N. Mikhailenko ${ }^{\mathrm{m}}$, N. Moazzen-Ahmadi ${ }^{\mathrm{y}}$, H.S.P. Müller ${ }^{\mathrm{z}}$, O.V. Naumenko ${ }^{\mathrm{m}}$, A.V. Nikitin ${ }^{\mathrm{m}}$, O.L. Polyansky ${ }^{\mathrm{b}, \mathrm{w}}$, M. Rey ${ }^{\mathrm{q}}$, M. Rotger ${ }^{\mathrm{q}}$, S.W. Sharpe ${ }^{\mathrm{u}}$, K. Sung ${ }^{\mathrm{h}}$, E. Starikova ${ }^{\mathrm{m}}$, S.A. Tashkun ${ }^{\mathrm{m}}$, J. Vander Auwera ${ }^{\text {aa }}$, G. Wagner ${ }^{\mathrm{e}}$, J. Wilzewski ${ }^{\mathrm{a}, \mathrm{e}}$, P. Wcisło ${ }^{\mathrm{bb}}$, S. Yu ${ }^{\mathrm{h}}$, E.J. Zak ${ }^{\mathrm{b}}$

${ }^{a}$ Harvard-Smithsonian Center for Astrophysics, Atomic and Molecular Physics Division, Cambridge, MA, USA

${ }^{\mathrm{b}}$ University College London, Dept. of Physics and Astronomy, London WC1E 6BT, UK

${ }^{\mathrm{c}}$ Tomsk State University, Laboratory of Quantum Mechanics of Molecules and Radiative Processes, Tomsk, Russia

d Old Dominion University, Dept. of Chemistry \& Biochemistry, Norfolk, VA, USA

e DLR, Institute for Remote Sensing Technology, Wessling, Germany

${ }^{\mathrm{f}}$ Université de Bourgogne Franche-Comté, Laboratoire Interdisciplinaire Carnot de Bourgogne, UMR 6303 CNRS, Dijon Cedex, France

g Université Grenoble, Grenoble, France

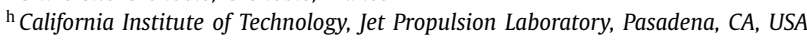

i Université Paris Est, LISA, Créteil, France

j University of Massachusetts, Dept. of Environmental, Earth \& Atmospheric Sciences, Lowell, MA, USA

${ }^{k}$ NIST, Chemical Sciences Division, Gaithersburg, MD, USA

${ }^{1}$ Université Pierre et Marie Curie, LADIR, Paris, France

${ }^{\mathrm{m}}$ Institute of Atmospheric Optics, Laboratory of Theoretical Spectroscopy, Tomsk, Russia

${ }^{n}$ CNRS, Ecole Polytechnique, Université Paris-Saclay, Laboratoire de Météorologie Dynamique/IPSL, 91128 Palaiseau, France

${ }^{\circ}$ University of Reading, Dept. of Meteorology, Reading, UK

${ }^{\mathrm{p}}$ NASA Langley Research Center, Science Directorate, Hampton, VA, USA

q Université de Reims, GSMA, Reims, France

${ }^{\mathrm{r}}$ MTA-ELTE Complex Chemical Systems Research Group, Budapest, Hungary

${ }^{s}$ The College of William and Mary, Dept. of Physics, Williamsburg, VA, USA

${ }^{\mathrm{t}}$ University of Leicester, Department of Physics and Astronomy, Leicester, UK

u Battelle Pacific Northwest National Laboratory, Richland, WA, USA

${ }^{v}$ Radboud University, Institute for Molecules and Materials, Nijmegen, The Netherlands

${ }^{\mathrm{w}}$ Institute of Applied Physics of Russian Academy of Sciences, Nizhny Novgorod, Russia

${ }^{x}$ University of Colorado, Laboratory for Atmospheric and Space Physics, Boulder, CO, USA

${ }^{y}$ The University of Calgary, Dept. of Physics and Astronomy, Calgary, AB, Canada

${ }^{2}$ University of Cologne, I. Physikalisches Institut, Cologne, Germany

aa Université Libre de Bruxelles, Service de Chimie Quantique et Photophysique, C.P. 160/09, B-1050 Brussels, Belgium

${ }^{b b}$ Nicolaus Copernicus University, Institute of Physics, Torun, Poland

${ }^{t t}$ University of Leicester, National Centre for Earth Observation, Leicester, UK

ttt University of Leicester, Leicester Institute for Space and Earth Observation, Leicester, UK

${ }^{\mathrm{rr}}$ Eötvös Loránd University, Institute of Chemistry, Budapest, Hungary

\footnotetext{
* Corresponding author.

E-mail address: IGordon@cfa.harvard.edu (I.E. Gordon).
} 


\section{A R T I C L E I N F O}

\section{Article history:}

Received 23 February 2017

Revised 29 June 2017

Accepted 29 June 2017

Available online $\mathrm{xxx}$

\section{Keywords:}

HITRAN

Spectroscopic database

Molecular spectroscopy

Molecular absorption

Spectroscopic line parameters

Absorption cross-sections

Collision-induced absorption

Aerosols

\begin{abstract}
A B S T R A C T
This paper describes the contents of the 2016 edition of the HITRAN molecular spectroscopic compilation. The new edition replaces the previous HITRAN edition of 2012 and its updates during the intervening years. The HITRAN molecular absorption compilation is composed of five major components: the traditional line-by-line spectroscopic parameters required for high-resolution radiative-transfer codes, infrared absorption cross-sections for molecules not yet amenable to representation in a line-by-line form, collision-induced absorption data, aerosol indices of refraction, and general tables such as partition sums that apply globally to the data. The new HITRAN is greatly extended in terms of accuracy, spectral coverage, additional absorption phenomena, added line-shape formalisms, and validity. Moreover, molecules, isotopologues, and perturbing gases have been added that address the issues of atmospheres beyond the Earth. Of considerable note, experimental IR cross-sections for almost 300 additional molecules important in different areas of atmospheric science have been added to the database. The compilation can be accessed through www.hitran.org. Most of the HITRAN data have now been cast into an underlying relational database structure that offers many advantages over the long-standing sequential text-based structure. The new structure empowers the user in many ways. It enables the incorporation of an extended set of fundamental parameters per transition, sophisticated line-shape formalisms, easy user-defined output formats, and very convenient searching, filtering, and plotting of data. A powerful application programming interface making use of structured query language (SQL) features for higher-level applications of HITRAN is also provided.
\end{abstract}

Published by Elsevier Ltd This is an open access article under the CC BY-NC-ND license. (http://creativecommons.org/licenses/by-nc-nd/4.0/)

\section{Introduction}

This article describes the data and software that have been added, modified, or enhanced in the HITRAN (High Resolution Transmission) compilation since the previous edition of 2012 [1] (hereafter called HITRAN2012 in the text). The line-by-line portion and the absorption cross-sections of the new edition, hereafter called HITRAN2016, have now been cast into an underlying relational database structure as opposed to the long-standing fixedlength ASCII record format. A user-friendly powerful internet tool, HITRANonline (accessible through www.hitran.org), is provided to the user to query, filter, and plot sections of the data and to retrieve outputs in a host of convenient formats (see Hill et al. [2]).

The HITRAN compilation is composed of several components that include (1) line-by-line spectroscopic parameters for high resolution molecular absorption and radiance calculations (from the microwave through the ultraviolet region of the spectrum); (2) infrared absorption cross-sections (generally representing absorption by molecules that have very dense spectra or many low-lying vibrational modes); (3) collision-induced absorption datasets; (4) tables of aerosol refractive indices; and (5) global data that apply in a general manner to the archive. The updates to these five portions of HITRAN as well as the new underlying system of data structure with accompanying internet user interface will be discussed in the following sections.

The new edition of the HITRAN database substantially increases the database's potential to accurately model radiative processes in the atmosphere of the Earth and other planets. Apart from improving the accuracy of the existing parameters, more parameters have been introduced, and new bands and isotopologues added. Two gases $\left(\mathrm{C}_{2} \mathrm{~N}_{2}\right.$ and $\left.\mathrm{COCl}_{2}\right)$ are introduced to the database for the first time. Finally, a very dramatic expansion of the cross-sectional part of the database is featured in this new edition.

It is necessary to call attention to some specifics of the HITRAN database. The units used throughout HITRAN editions including this one do not strictly adhere to the SI system for both historical and application-specific reasons. Thus $\mathrm{cm}^{-1}$ (reciprocal centimeter) is seen throughout, as is atm (atmosphere) for pressure (in SI units of Pascals, $101,325 \mathrm{~Pa}=1 \mathrm{~atm}$ ). Also, the symbol $v$ is used throughout for line position in $\mathrm{cm}^{-1}$, thereby dropping the tilde $(\tilde{v})$ that is the official designation of wavenumber. The HITRAN unit for intensity is traditionally expressed as $\mathrm{cm}^{-1} /\left(\right.$ molecule $\left.\mathrm{cm}^{-2}\right)$ rather than simplifying to the equivalent $\mathrm{cm}$ molecule ${ }^{-1}$.

\subsection{Overview of parameters (including new ones)}

The traditional and probably most applied portion of HITRAN is the line list of high-resolution spectral parameters. These line-byline parameters reflect values of individual transitions between energy levels of rovibronic states that are required by high-resolution radiative-transfer codes. These parameters are shown in Tables 1 and 2. Table 1 gives an overview of parameters that are traditionally provided in the ".par" format as per HITRAN2004 [3] formalism as well as broadening and shift parameters due to the pressure of $\mathrm{H}_{2}, \mathrm{He}$ and $\mathrm{CO}_{2}$ (see Wilzewski et al. [4], Hill et al. [2] and Kochanov et al. [5] for details). Table 2 provides information on the parameters required for non-Voigt line-shape representations (see Wcisło et al. [6], Hill et al. [2] and Kochanov et al. [5] for details). Ideally, they would arise from theoretically-consistent calculations; however, since the emphasis in HITRAN is to provide the user with the most accurate values available, they are often the values obtained from controlled laboratory measurements when quantummechanical calculations are not yet of comparable accuracy. Nevertheless, theoretical calculations and semi-empirical formulae are widely used in HITRAN to both interpolate and extrapolate needed parameters for transitions missing from the limited observed set.

Section 2 presents a description of changes made to the lineby-line portion of HITRAN. It is organized in the order of the molecules in HITRAN (a sequential number related to the entry of the molecule into HITRAN). The discussions in the molecular sub-sections give an overview of the addition of new bands, replacement of line positions and/or intensities, and new or modified line-shape parameters where applicable. There are also citations to more detailed studies of the modifications.

With the recent advances in both laboratory spectrometers and the power of theoretical treatments such as ab initio calculations, the accuracy and the access to weak, yet important, transitions have had a big impact on this edition of HITRAN.

Table 3 provides an overview of changes and additions to the database with respect to the HITRAN2012 edition.

The line-by-line parameters (as well as the portion of HITRAN devoted to cross-sections) have now been cast into a relational database structure, described in Section 6.1. This underlying structure has many advantages that were not available in the previous fixed-length format of previous HITRAN editions. The database is now able to add many more parameters (becoming necessary 
Table 1

Parameter identifiers in HITRANonline.

\begin{tabular}{|c|c|c|c|}
\hline \multicolumn{4}{|c|}{ Parameters from the HITRAN2004 format in HITRANonline } \\
\hline Molecule ID & - & molec_id & HITRAN integer identifying the molecule \\
\hline Isotopologue ID & - & local_iso_id & HITRAN integer identifying the isotopologue of a particular molecule \\
\hline$v$ & $\mathrm{~cm}^{-1}$ & nu & Transition wavenumber \\
\hline$S$ & $\mathrm{~cm}^{-1} /\left(\right.$ molecule $\left.\mathrm{cm}^{-2}\right)$ & sw & Transition intensity, weighted by isotopologue abundance \\
\hline$A$ & $\mathrm{~s}^{-1}$ & a & Einstein $A$-coefficient \\
\hline$\gamma_{\text {air }}$ & $\mathrm{cm}^{-1} \mathrm{~atm}^{-1}$ & gamma_air & $\begin{array}{l}\text { Air-broadened Lorentzian half width at half maximum (HWHM) } \\
\text { coefficient (for Voigt lineshape) }\end{array}$ \\
\hline$\gamma_{\text {self }}$ & $\mathrm{cm}^{-1} \mathrm{~atm}^{-1}$ & gamma_self & Self-broadened Lorentzian HWHM coefficient (for Voigt lineshape) \\
\hline$E^{\prime \prime}$ & $\mathrm{cm}^{-1}$ & elower & Lower-state energy \\
\hline$n_{\text {air }}$ & - & n_air & Temperature-dependence exponent for $\gamma_{\text {air }}$ \\
\hline$\delta_{\text {air }}$ & $\mathrm{cm}^{-1} \mathrm{~atm}^{-1}$ & delta_air & Air pressure-induced line shift \\
\hline$V^{\prime} b$ & - & global_upper_quanta & Upper-state "global" quanta in HITRAN2004 format \\
\hline$V^{\prime \prime} b$ & - & global_lower_quanta & Lower-state "global" quanta in HITRAN2004 format \\
\hline$Q^{\prime}{ }^{b}$ & - & local_upper_quanta & Upper-state “local” quanta in HITRAN2004 format \\
\hline$Q^{\prime \prime} b$ & - & local_lower_quanta & Lower-state "local" quanta in HITRAN2004 format \\
\hline Ierr ${ }^{b}$ & & ierr & $\begin{array}{l}\text { Ordered list of indices corresponding to uncertainty estimates of } \\
\text { transition parameters }\end{array}$ \\
\hline Iref $b$ & & iref & Ordered list of reference identifiers for transition parameters \\
\hline$g^{\prime}$ & - & gp & Upper-state statistical weight \\
\hline$g^{\prime \prime}$ & - & gpp & Lower-state statistical weight \\
\hline \multicolumn{4}{|c|}{ Metadata and other special parameters } \\
\hline Transition ID & - & trans_id & Unique integer identifying the transition \\
\hline Global Isotopologue ID & - & global_iso_id & $\begin{array}{l}\text { Global integer ID identifying the isotopologue (unique across the } \\
\text { whole database) }\end{array}$ \\
\hline qns' & - & statep & Upper-state quantum numbers in HITRANonline format \\
\hline qns" & - & statepp & Lower-state quantum numbers in HITRANonline format \\
\hline .par line & - & par_line & $\begin{array}{l}\text { Complete representation of the line in the HITRAN2004 160-character } \\
\text { format }\end{array}$ \\
\hline \multicolumn{4}{|c|}{ Parameters for broadening by new perturbing species, $X^{c}$, and line-mixing parameters } \\
\hline$\gamma_{X}$ & $\mathrm{~cm}^{-1} \mathrm{~atm}^{-1}$ & gamma_X & $\begin{array}{l}\text { Lorentzian HWHM coefficient (for Voigt lineshape) for broadening by } \\
\text { perturber } X\end{array}$ \\
\hline$n_{X}$ & - & $\mathrm{n} \_\mathrm{X}$ & Temperature-dependence exponent for $\gamma_{X}$ \\
\hline$n_{\text {self }}$ & - & n_self & Temperature-dependence exponent for $\gamma_{\text {self }}$ \\
\hline$\delta_{\text {self }}$ & $\mathrm{cm}^{-1} \mathrm{~atm}^{-1}$ & delta_self & Self-induced pressure line shift \\
\hline$\delta^{\prime}$ air & $\mathrm{cm}^{-1} \mathrm{~atm}^{-1} \mathrm{~K}^{-1}$ & deltap_air & Linear temperature dependence coefficient for $\delta_{\text {air }}$ \\
\hline$\delta^{\prime}$ self & $\mathrm{cm}^{-1} \mathrm{~atm}^{-1} \mathrm{~K}^{-1}$ & deltap_self & Linear temperature dependence coefficient for $\delta_{\text {self }}$ \\
\hline$\delta_{\mathrm{H}_{2}}^{\prime}$ & $\mathrm{cm}^{-1} \mathrm{~atm}^{-1} \mathrm{~K}^{-1}$ & deltap_H2 & Linear temperature dependence coefficient for $\delta_{\mathrm{H}_{2}}$ \\
\hline$\delta_{X}^{n_{2}}$ & $\mathrm{~cm}^{-1} \mathrm{~atm}^{-1}$ & delta_X & Pressure-induced line shift due to perturber $X$ \\
\hline$Y_{\text {air }}$ & $\mathrm{cm}^{-1} \mathrm{~atm}^{-1}$ & y_air & $\begin{array}{l}\text { First-order (Rosenkranz) line coupling coefficient within Voigt profile; } \\
\text { air-broadened environment }\end{array}$ \\
\hline$Y_{\text {self }}$ & $\mathrm{cm}^{-1} \mathrm{~atm}^{-1}$ & y_self & $\begin{array}{l}\text { First-order (Rosenkranz) line coupling coefficient within Voigt profile; } \\
\text { air-broadened environment }\end{array}$ \\
\hline
\end{tabular}

a The third column header, identifier, denotes the names of the variables used in the new relational database structure. ${ }^{b}$ These parameters are given in Table 1 in the

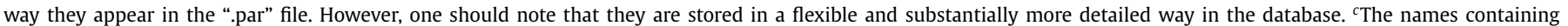

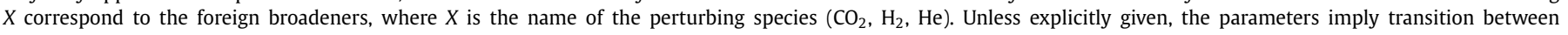
lower and upper states; double primes (") and primes (') are for lower and upper energy states, respectively.

not only for planetary atmosphere applications, but also to satisfy sophisticated remote-sensing requirements), is not limited to constraints of field length, and can accommodate functional forms for parameters. It also allows for a much more robust way for the managers to check for errors, whether they be by transcription, violated selection rules, incorrect quantum assignments, etc.

\subsection{New line-shape representations}

Shapes of individual transitions in molecular spectra at atmospheric conditions are mainly determined by collisions and the Doppler effect. In the simplest case these two effects can be handled with the Voigt profile, where the collisions are described just as a single-parameter exponential relaxation. In the previous editions of HITRAN, the line shapes, with a very few exceptions, were represented with the Voigt profile. It turns out, however, that this model is too simple to reproduce atmospheric spectra with accuracy at the sub-percent level (see for instance Refs. $[9,10])$. For some light molecules, in particular for molecular hydrogen (important for research on dense atmospheres of gas giants), the non-Voigt effects are much more pronounced [6]. At this point the database provides Voigt (for every transition), and speed- dependent Voigt, Galatry [11] and Hartmann-Tran [7,8] profiles parameters where they had been measured and validated.

$\mathrm{Ab}$ initio line-shape models, originating from first principles, are far too complex to be simply implemented in a line-by-line spectroscopic database. Therefore, a compromise between simplicity of the model and the fidelity of experimental spectra representation had to be found. In recent decades, a number of phenomenological models that take into account both speeddependent effects and velocity-changing collisions had been advanced. Recently, Tran et al. developed a computationally efficient algorithm $[7,8]$ for calculating one of them, the partially-correlated speed-dependent hard-collision profile [12] with quadratic speed dependence [9]. An IUPAC (International Union of Pure and Applied Chemistry) task group recommended the use of this profile for high-resolution spectra representation [13] and call it the Hartmann-Tran (HT) profile. Ngo et al. [14] demonstrated that the HT profile well reconstructs the line shapes of the most important atmospheric molecules. Therefore, the HT profile has been adopted as the default beyond-Voigt profile in HITRAN. For measurements with sufficiently high accuracy, we encourage spectroscopists to report not only the basic Voigt-profile parameters, but also the HT parameters. The details about the HT parametrization adopted in 
Table 2

Parameter identifiers in HITRANonline for non-Voigt parameters.

\begin{tabular}{|c|c|c|c|}
\hline \multicolumn{4}{|c|}{ Parameters for non-Voigt parameters } \\
\hline Parameter & Units & Identifier $^{\mathrm{a}}$ & Description \\
\hline$\gamma_{0}^{S D V}(X ; 296)$ & $\mathrm{cm}^{-1} \mathrm{~atm}^{-1}$ & gamma_SDV_0_X_296 & Air-(Self-) broadened Lorentzian HWHM coefficient in $296 \mathrm{~K}$ (for SDV profile) \\
\hline$\gamma_{2}^{S D V}(X ; 296)$ & $\mathrm{cm}^{-1} \mathrm{~atm}^{-1}$ & gamma_SDV_2_X_296 & Speed-dependence of the SDV halfwidth in $296 \mathrm{~K}$; air-(self-) broadened case \\
\hline$\delta_{0}^{S D V}(X ; 296)$ & $\mathrm{cm}^{-1} \mathrm{~atm}^{-1}$ & delta_SDV_0_X_296 & Air-(Self-) induced pressure line shift in $296 \mathrm{~K}$ (for SDV profile) \\
\hline$\delta^{\prime} S D V(X ; 296)$ & $\mathrm{cm}^{-1} \mathrm{~atm}^{-1} \mathrm{~K}^{-1}$ & deltap_SDV_X_296 & Linear temperature dependence coefficient for $\delta_{0}^{S D V}(X ; 296)$ \\
\hline$n^{S D V}(X ; 296)$ & - & n_SDV_X_296 & Temperature dependence exponent around $296 K$ for $\gamma_{0}^{S D V}(X ; 296)$ \\
\hline$Y_{X}^{S D V}(296)$ & $\mathrm{cm}^{-1} \mathrm{~atm}^{-1}$ & Y_SDV_X_296 & First-order (Rosenkranz) line coupling coefficient within SDV ${ }^{b}$ profile; air-(self-) broadened case \\
\hline$\gamma_{X}^{G}$ & $\mathrm{~cm}^{-1} \mathrm{~atm}^{-1}$ & gamma_g_X & Air-(Self-) broadened Lorentzian HWHM coefficient in $296 \mathrm{~K}$ (for Galatry profile) \\
\hline$\beta_{X}^{G}$ & $\mathrm{~cm}^{-1} \mathrm{~atm}^{-1}$ & beta_g_X & Dicke narrowing parameter for the air- (self-) broadened Galatry line profile \\
\hline$\gamma_{0}^{H T}\left(X ; T_{\text {ref }}\right)$ & $\mathrm{cm}^{-1} \mathrm{~atm}^{-1}$ & gamma_HT_0_X_T & Speed-averaged HTP halfwidth in temperature range around $T=T_{\text {ref }}$ d due to perturber $X$ \\
\hline$n^{H T}\left(X ; T_{r e f}\right)$ & - & n_HT_X_T & Temperature dependence exponent in the range corresponding to $T=T_{\text {ref }}$ for $\gamma_{0}^{H T}\left(X ; T_{r e f}\right)$ \\
\hline$\gamma_{2}^{H T}\left(X ; T_{r e f}\right)$ & $\mathrm{cm}^{-1} \mathrm{~atm}^{-1}$ & gamma_HT_2_X_T & Speed-dependence of the HTP halfwidth in temperature range around $T=T_{\text {ref }}$ due to perturber $X$. \\
\hline$\delta_{0}^{H T}\left(X ; T_{r e f}\right)$ & $\mathrm{cm}^{-1} \mathrm{~atm}^{-1}$ & delta_HT_0_X_T & Speed-averaged line shift of the HTP in temperature range around $T=T_{\text {ref }}$ due to perturber $X$ \\
\hline$\delta^{\prime H T}\left(X ; T_{r e f}\right)$ & $\mathrm{cm}^{-1} \mathrm{~atm}^{-1} \mathrm{~K}^{-1}$ & deltap_HT_0_X_T & Linear temperature dependence coefficient for $\delta_{0}^{H T}\left(X ; T_{\text {ref }}\right)$ in temperature range around $T=T_{\text {ref }}$ \\
\hline$\delta_{2}^{H T}\left(X ; T_{r e f}\right)$ & $\mathrm{cm}^{-1} \mathrm{~atm}^{-1}$ & delta_HT_2_X_T & Speed-dependence of the HTP line shift in temperature range around $T=T_{\text {ref }}$ due to perturber $X$ \\
\hline$v_{V C}^{H T}(X)$ & $\mathrm{cm}^{-1} \mathrm{~atm}^{-1}$ & nu_HT_X & Frequency of velocity changing collisions in the HT profile formalism \\
\hline$\kappa^{H T}(X)$ & - & kappa_HT_X & Temperature dependence of $v_{V C}^{H T}(X)$ \\
\hline$\eta^{H T}$ & - & eta_HT_X & Correlation parameter in HT profile formalism \\
\hline$Y^{H T}(X ; 296)$ & $\mathrm{cm}^{-1} \mathrm{~atm}^{-1}$ & Y_HT_X_296 & First-order (Rosenkranz) line coupling coefficient within HT profile; air-(self-) broadened case \\
\hline
\end{tabular}

a The third column header, identifier, denotes the names of the variables used in the new relational database structure. The names containing the $X$ literal correspond to air- or self- broadening

b SDV stands for speed-dependent Voigt.

c HT stands for the Hartmann-Tran profile $[7,8]$.

${ }^{\mathrm{d}}$ For the four temperature ranges and corresponding $T_{\text {ref }}$ values consult Wcisło et al. [6] and Sections 1.2 and 2.45.

HITRAN are given in Wcisło et al. [6]. Apart from new parameters introduced to represent this profile, one should note that these parameters are also provided at four different temperature ranges: 0 $100 \mathrm{~K}, 100-200 \mathrm{~K}, 200-400 \mathrm{~K}$ and $\mathrm{T}>400 \mathrm{~K}$. The reference parameters are given at the following reference temperatures: $50 \mathrm{~K}, 150 \mathrm{~K}$, $296 \mathrm{~K}$ and $700 \mathrm{~K}$ respectively.

It should be noted that even if the parameters of some more advanced model (preferably HT) are now provided in HITRAN for a growing number of transitions, the Voigt-profile parameters are still given for every transition in HITRAN. Therefore, for users not interested in making use of sophisticated line shapes, the present version of HITRAN remains compatible with the previous ones.

\section{LIne-by-line modifications}

\section{1. $\mathrm{H}_{2} \mathrm{O}$ (molecule 1)}

The HITRAN2016 edition has undergone a substantial revision and expansion of the database of water vapor.

In HITRAN2012 [1] a very large expansion of the dynamic range of the line intensities for non-deuterated isotopologues of water vapor was implemented, thanks to the ab initio calculations from the BT2 line list [15] and, in selected spectral intervals, Lodi et al. [16] for the principal isotopologue and from Lodi and Tennyson for $\mathrm{H}_{2}{ }^{18} \mathrm{O}$ and $\mathrm{H}_{2}{ }^{17} \mathrm{O}$ [17]. The ab initio results were replaced with high-quality experimental or-semi-empirical data wherever possible. In HITRAN2016, we have done a similar expansion of the dynamic (and spectral) range of the singly-deuterated isotopologues. In addition, the $\mathrm{D}_{2}{ }^{16} \mathrm{O}$ isotopologue makes its debut in the database. $\mathrm{D}_{2} \mathrm{O}$ has a very low natural abundance on Earth but is measurable in the atmospheres of other planets, notably Venus which has an enhanced deuterium content [18] raising the importance of $\mathrm{D}_{2} \mathrm{O}$. Also, for fitting D-enriched HDO laboratory spectra, a good $\mathrm{D}_{2} \mathrm{O}$ line list is essential. For this reason, $\mathrm{D}_{2} \mathrm{O}$ transitions have been included using a lower intensity cutoff of $10^{-32}$ $\mathrm{cm}^{-1} /\left(\right.$ molecule $\cdot \mathrm{cm}^{-2}$ ) once isotopic abundance is accounted for.

A dedicated paper detailing the update of the water-vapor dataset in HITRAN2016 along with atmospheric and laboratory validations is planned. Here we summarize only the most important details.

\subsubsection{Line positions and intensities}

This update is informed by the work of an International Union of Pure and Applied Chemistry (IUPAC) task group which produced systematic sets of empirical energy levels (and hence transition frequencies) for all the stable isotopologues of water [19-22]. In HITRAN2012 non-deuterated isotopologues already benefited from the availability of the IUPAC dataset of empirically-derived energy levels (and transition wavenumbers derived from them), and we extend this to the deuterated species here. However, some important caveats of that compilation have to be noted.

a) The accuracy of some of the transitions generated from the IUPAC set may be inferior to individual high-accuracy experiments. Therefore, just like in HITRAN2012, we have given preference to the experimental line position data from previous HITRAN editions if the uncertainty code was $5(0.00001-$ $0.0001 \mathrm{~cm}^{-1}$ ) or higher. Comparisons with atmospheric spectra have shown that this was a correct choice.

b) The datasets constructed in Refs. [19-22] do not include experimental data from the papers published after them. Quite a few new levels have become available over the years and some reassignments were in order (see for instance Mikhailenko et al. [23] and Liu et al. [24]). We partially updated the IUPAC datasets here. In particular, for the $\mathrm{HD}^{17} \mathrm{O}$ and $\mathrm{HD}^{18} \mathrm{O}$ species, levels from Kyuberis et al. [25] were used. For $\mathrm{H}_{2}{ }^{18} \mathrm{O}$ and $\mathrm{H}_{2}{ }^{17} \mathrm{O}$, and to a lesser extent for $\mathrm{H}_{2}{ }^{16} \mathrm{O}$, levels from Mikhailenko et al. $[23,26]$ and Liu et al. [24] were used. Unfortunately, the IUPAC and Mikhailenko et al. quantum assignments often differ and complete matching would require a substantial amount of time in the future. An update of the IUPAC energy levels is in progress which will endeavor to resolve these assignment issues.

Many of the updated water vapor lines use line positions generated from the IUPAC energy levels and theoretical transition intensities, based on a high-accuracy ab initio dipole moment surface [16]. The methodology developed by Lodi and Tennyson [17] involves using several calculated line lists to identify reliable theoretical predictions. Lodi and Tennyson's intensity data were used in their entirety for $\mathrm{H}_{2}{ }^{17} \mathrm{O}$ and $\mathrm{H}_{2}{ }^{18} \mathrm{O}$ in HITRAN2012. Subsequent measurements and analysis by Regalia et al. [27,28] gave good 
Table 3

Molecules and isotopologues represented in line-by-line portion of HITRAN.

\begin{tabular}{|c|c|c|c|c|c|c|c|}
\hline Molecule & Isotopologue $^{\mathrm{a}}$ & $\begin{array}{l}\text { HITRAN2012 } \\
\text { Spectral Coverage } \\
\left(\mathrm{cm}^{-1}\right)\end{array}$ & $\begin{array}{l}\text { HITRAN2016 } \\
\text { Spectral Coverage } \\
\left(\mathrm{cm}^{-1}\right)\end{array}$ & $\begin{array}{l}\text { HITRAN2012 } \\
\text { Number of } \\
\text { Transitions }\end{array}$ & $\begin{array}{l}\text { HITRAN2016 } \\
\text { Number of } \\
\text { Transitions }\end{array}$ & $\begin{array}{l}\text { Non-Voigt line } \\
\text { shape for at } \\
\text { least some } \\
\text { transitions? }\end{array}$ & $\begin{array}{l}\text { Broadening by } \\
\mathrm{H}_{2} \text {, He and } \mathrm{CO}_{2}\end{array}$ \\
\hline \multirow[t]{7}{*}{ (1) $\mathbf{H}_{2} \mathbf{O}$} & 161 & $0-25,711$ & $0-25,711$ & 142045 & $140751^{\mathrm{d}}$ & Yes & \\
\hline & $181^{\mathrm{b}}$ & $0-19,918$ & $0-19,918$ & 39903 & 39901 & & \\
\hline & $171^{\mathrm{b}}$ & $0-19,946$ & $0-19,946$ & 27544 & 27544 & & \\
\hline & 162 & $0-22,708$ & $0-22,708$ & 13237 & 56430 & & \\
\hline & 182 & $0-3825$ & $0-10,729$ & 1611 & 10664 & & \\
\hline & 172 & $1234-1599$ & $0-10,703$ & 175 & 6313 & & \\
\hline & 262 & $\mathrm{c}$ & $0-12,812$ & c & 32184 & & \\
\hline \multirow[t]{12}{*}{ (2) $\mathrm{CO}_{2}$} & 626 & $345-12,785$ & $158-14,076$ & 169292 & 173024 & & \\
\hline & 636 & $406-12,463$ & $332-13,735$ & 70611 & 70577 & & \\
\hline & 628 & $0-9558$ & $1-12,678$ & 116482 & 127850 & & \\
\hline & 627 & $0-9600$ & $0-12,727$ & 72525 & 77941 & & \\
\hline & 638 & $489-6745$ & $2-9213$ & 26737 & 43782 & & \\
\hline & 637 & $583-6769$ & $9-8062$ & 2953 & 25175 & & \\
\hline & 828 & $491-8161$ & $482-8163$ & 7118 & 10522 & & \\
\hline & 827 & $626-5047$ & $491-8194$ & 821 & 15878 & & \\
\hline & 727 & $535-6933$ & $535-6933$ & 5187 & 6518 & & \\
\hline & 838 & $4599-4888$ & $2245-4751$ & 121 & 2916 & & \\
\hline & 837 & c & $549-4915$ & c & 4190 & & \\
\hline & 737 & c & $575-3615$ & c & 1501 & & \\
\hline \multirow[t]{5}{*}{ (3) $\mathbf{O}_{3}$} & 666 & $0-6997$ & $0-6997$ & 261886 & 289340 & & \\
\hline & 668 & $0-2768$ & $0-2768$ & 44302 & 44302 & & \\
\hline & 686 & $1-2740$ & $1-2740$ & 18887 & 18887 & & \\
\hline & 667 & $0-2122$ & $0-2122$ & 65106 & 65106 & & \\
\hline & 676 & $0-2101$ & $0-2101$ & 31935 & 31935 & & \\
\hline \multirow[t]{5}{*}{ (4) $\mathbf{N}_{2} \mathbf{O}$} & $446^{\mathrm{b}}$ & $0-7797$ & $0-7797$ & 33074 & 33074 & Yes & \\
\hline & 456 & $5-5086$ & $5-5086$ & 4222 & 4222 & & \\
\hline & 546 & $4-4704$ & $4-4704$ & 4592 & 4592 & & \\
\hline & 448 & $542-4672$ & $0-4672$ & 10364 & 116694 & & \\
\hline & 447 & $550-4430$ & $550-4430$ & 1705 & 1705 & & \\
\hline \multirow[t]{6}{*}{ (5) CO } & 26 & $3-8465$ & $3-14,478$ & 1019 & 1344 & Yes & Yes \\
\hline & 36 & $3-6279$ & $3-12,231$ & 797 & 1042 & Yes & Yes \\
\hline & 28 & $3-6267$ & $3-12,205$ & 770 & 920 & Yes & Yes \\
\hline & 27 & $3-6339$ & $3-10,295$ & 728 & 800 & & Yes \\
\hline & 38 & $3-6124$ & $3-8078$ & 712 & 674 & & Yes \\
\hline & 37 & $1807-6197$ & $3-8168$ & 580 & 601 & & Yes \\
\hline \multirow[t]{4}{*}{ (6) $\mathbf{C H}_{4}$} & 211 & $0-11,502$ & $0-11,502$ & 336829 & 313943 & & \\
\hline & 311 & $0-11,319$ & $0-11,319$ & 72420 & 77626 & & \\
\hline & 212 & $15-6511$ & $7-6511$ & 54550 & 54550 & & \\
\hline & 312 & $959-1695$ & $959-1695$ & 4213 & 4213 & & \\
\hline \multirow[t]{3}{*}{ (7) $\mathbf{O}_{2}$} & 66 & $0-15,928$ & $0-57,028$ & 1787 & 15263 & Yes & \\
\hline & 68 & $1-15,853$ & $1-56,670$ & 875 & 2965 & Yes & \\
\hline & $67^{b}$ & $0-14,538$ & $0-14,537$ & 11313 & 11313 & Yes & \\
\hline \multirow[t]{3}{*}{ (8) NO } & 46 & $0-9274$ & $0-9274$ & 103701 & $103701^{\mathrm{d}}$ & & \\
\hline & 56 & $1609-2061$ & $1609-2061$ & 699 & 699 & & \\
\hline & 48 & $1602-2039$ & $1602-2039$ & 679 & 679 & & \\
\hline \multirow[t]{2}{*}{ (9) $\mathrm{SO}_{2}$} & $626^{\mathrm{b}}$ & $0-4093$ & $0-4093$ & 72460 & 72460 & & Yes \\
\hline & $646^{\mathrm{b}}$ & $0-2501$ & $0-2501$ & 22661 & 22661 & & Yes \\
\hline (10) $\mathrm{NO}_{2}$ & 646 & $0-3075$ & $0-3075$ & 104223 & 104223 & & \\
\hline \multirow[t]{2}{*}{ (11) $\mathrm{NH}_{3}$} & 446 & $0-7000$ & $0-10,349$ & 45302 & 65828 & & Yes \\
\hline & 456 & $0-5180$ & $0-5180$ & 1090 & 1320 & & Yes \\
\hline (12) $\mathrm{HNO}_{3}$ & 146 & $0-1770$ & $0-1770$ & 903854 & 950864 & & \\
\hline & 156 & $0-923$ & $0-923$ & 58108 & 58108 & & \\
\hline (13) $\mathbf{O H}$ & 61 & $0-19,268$ & $0-19,268$ & 30772 & 30772 & & \\
\hline & 81 & $0-329$ & $0-329$ & 295 & 295 & & \\
\hline & 62 & $0-332$ & $0-332$ & 912 & 912 & & \\
\hline (14) HF & 19 & $24-46,985$ & $24-32,352$ & 10073 & 8090 & Yes & Yes \\
\hline & 29 & $13-47,365$ & $13-20,830$ & 24303 & 11920 & & Yes \\
\hline (15) $\mathbf{H C l}$ & 15 & $8-34,250$ & $8-20,321$ & 11879 & 8892 & Yes & Yes \\
\hline & 17 & $8-34,240$ & $8-20,219$ & 11907 & 8908 & Yes & Yes \\
\hline & 25 & $5-33,284$ & $5-15,266$ & 29994 & 17762 & & Yes \\
\hline & 27 & $5-33,258$ & $5-15,247$ & 29911 & 17690 & & Yes \\
\hline (16) $\mathbf{H B r}$ & 19 & $13-16,034$ & $13-16,034$ & 3039 & 3039 & & \\
\hline & 11 & $13-16,032$ & $13-16,032$ & 3031 & 3031 & & \\
\hline & 29 & $7-8781$ & $7-8781$ & 1455 & 1455 & & \\
\hline & 21 & $7-8778$ & $7-8778$ & 1455 & 1455 & & \\
\hline (17) HI & 17 & $10-13,908$ & $10-13,908$ & 3161 & 3161 & & \\
\hline & 27 & $5-7625$ & $5-7625$ & 1590 & 1590 & & \\
\hline (18) ClO & 56 & $0-1208$ & $0-1208$ & 5721 & 5721 & & \\
\hline & 76 & $0-1200$ & $0-1200$ & 5780 & 5780 & & \\
\hline (19) OCS & 622 & $0-4200$ & $0-7822$ & 15618 & 18264 & & Yes \\
\hline
\end{tabular}


Table 3 (continued)

\begin{tabular}{|c|c|c|c|c|c|c|c|}
\hline Molecule & Isotopologue & $\begin{array}{l}\text { HITRAN2012 } \\
\text { Spectral Coverage } \\
\left(\mathrm{cm}^{-1}\right)\end{array}$ & $\begin{array}{l}\text { HITRAN2016 } \\
\text { Spectral Coverage } \\
\left(\mathrm{cm}^{-1}\right)\end{array}$ & $\begin{array}{l}\text { HITRAN2012 } \\
\text { Number of } \\
\text { Transitions }\end{array}$ & $\begin{array}{l}\text { HITRAN2016 } \\
\text { Number of } \\
\text { Transitions }\end{array}$ & $\begin{array}{l}\text { Non-Voigt line } \\
\text { shape for at } \\
\text { least some } \\
\text { transitions? }\end{array}$ & $\begin{array}{l}\text { Broadening by } \\
\mathrm{H}_{2} \text {, He and } \mathrm{CO}_{2}\end{array}$ \\
\hline & 624 & $0-4166$ & $0-7796$ & 6087 & 6846 & & Yes \\
\hline & 632 & $0-4056$ & $0-6660$ & 3129 & 3275 & & Yes \\
\hline & 623 & $0-4164$ & $0-6631$ & 2886 & 3005 & & Yes \\
\hline & 822 & $0-4046$ & $0-4046$ & 1641 & 1640 & & Yes \\
\hline \multirow[t]{3}{*}{ (20) $\mathbf{H}_{2} \mathbf{C O}$} & 126 & $0-3100$ & $0-3100$ & 40670 & 40670 & & \\
\hline & 136 & $0-117$ & $0-117$ & 2309 & 2309 & & \\
\hline & 128 & $0-101$ & $0-101$ & 1622 & 1622 & & \\
\hline \multirow[t]{2}{*}{ (21) HOCl } & $165^{\mathrm{b}}$ & $1-3800$ & $1-3800$ & 8877 & 8877 & & \\
\hline & $176^{\mathrm{b}}$ & $1-3800$ & $1-3800$ & 7399 & 7399 & & \\
\hline \multirow[t]{2}{*}{ (22) $\mathbf{N}_{\mathbf{2}}$} & 44 & $11-9355$ & $11-9355$ & 1107 & 1107 & & \\
\hline & $45^{\mathrm{b}}$ & $11-2578$ & $11-2578$ & 161 & 161 & & \\
\hline \multirow[t]{3}{*}{ (23) HCN } & 124 & $0-3424$ & $0-17,586$ & 2955 & 58108 & & \\
\hline & 134 & $2-3405$ & $2-3405$ & 652 & 652 & & \\
\hline & 125 & $2-3420$ & $2-3420$ & 646 & 646 & & \\
\hline \multirow[t]{2}{*}{ (24) $\mathrm{CH}_{3} \mathrm{Cl}$} & 215 & $0-3198$ & $0-3198$ & 107642 & 110462 & & \\
\hline & 217 & $0-3198$ & $0-3198$ & 104854 & 109113 & & \\
\hline (25) $\mathbf{H}_{2} \mathbf{O}_{2}$ & $1661^{\mathrm{b}}$ & $0-1731$ & $0-1731$ & 126983 & 126983 & & \\
\hline \multirow[t]{3}{*}{ (26) $\mathbf{C}_{2} \mathbf{H}_{2}$} & 1221 & $604-9890$ & $13-9890$ & 12613 & 22866 & & Yes \\
\hline & 1231 & $613-6589$ & $613-6589$ & 285 & 285 & & Yes \\
\hline & 1222 & $1-789$ & $1-789$ & 7512 & 7512 & & Yes \\
\hline \multirow[t]{2}{*}{ (27) $\mathrm{C}_{2} \mathrm{H}_{6}$} & 1221 & $706-3001$ & $225-3001$ & 43592 & 54460 & & \\
\hline & 1231 & $725-919$ & $285-919$ & 6037 & 7107 & & \\
\hline (28) $\mathbf{P H}_{3}$ & 1111 & $0-3602$ & $0-3602$ & 22190 & 22190 & & \\
\hline \multirow[t]{2}{*}{ (29) $\mathrm{COF}_{2}$} & 269 & $696-2002$ & $725-2002$ & 168793 & 168793 & & \\
\hline & 369 & $686-815$ & $686-815$ & 15311 & 15311 & & \\
\hline (30) $\mathbf{S F}_{6}$ & 29 & $580-996$ & $580-996$ & 2889065 & 2889065 & & \\
\hline \multirow{3}{*}{ (31) $\mathbf{H}_{2} \mathbf{S}$} & $121^{\mathrm{b}}$ & $2-11,330$ & $2-11,330$ & 36561 & 36561 & & \\
\hline & $141^{\mathrm{b}}$ & $5-11,227$ & $5-11,227$ & 11352 & 11352 & & \\
\hline & $131^{\mathrm{b}}$ & $5-11,072$ & $5-11,072$ & 6322 & 6322 & & \\
\hline$(32)$ & 126 & $10-1890$ & $10-1890$ & 62684 & 62684 & & \\
\hline \multicolumn{8}{|l|}{ НСоОН } \\
\hline (33) $\mathbf{H O}_{2}$ & $166^{\mathrm{b}}$ & $0-3676$ & $0-3676$ & 38804 & 38804 & & \\
\hline (34) 0 & $6^{\mathrm{b}}$ & $68-159$ & $68-159$ & 2 & 2 & & \\
\hline (35) & 5646 & $763-798$ & $763-798$ & 21988 & 21988 & & \\
\hline \multicolumn{8}{|l|}{$\mathrm{ClONO}_{2}$} \\
\hline & 7646 & $765-791$ & $765-791$ & 10211 & 10211 & & \\
\hline (36) $\mathrm{NO}^{+}$ & 46 & $1634-2531$ & $3-2531$ & 1206 & 1270 & & \\
\hline \multirow[t]{2}{*}{ (37) $\mathrm{HOBr}$} & 169 & $0-316$ & $0-316$ & 2177 & 2177 & & \\
\hline & 161 & $0-316$ & $0-316$ & 2181 & 2181 & & \\
\hline \multirow[t]{2}{*}{ (38) $\mathbf{C}_{2} \mathbf{H}_{4}$} & 221 & $701-3243$ & $620-3243$ & 18097 & 59536 & & \\
\hline & 231 & $2947-3181$ & $614-3181$ & 281 & 18095 & & \\
\hline (39) $\mathrm{CH}_{3} \mathrm{OH}$ & $2161^{\mathrm{b}}$ & $0-1408$ & $0-1408$ & 19897 & 19897 & & \\
\hline \multirow[t]{2}{*}{ (40) $\mathbf{C H}_{3} \mathbf{B r}$} & 219 & $794-1706$ & $794-1706$ & 18692 & 18692 & & \\
\hline & 211 & $796-1697$ & $796-1697$ & 18219 & 18219 & & \\
\hline (41) $\mathbf{C H}_{3} \mathbf{C N}$ & 2124 & $890-946$ & $890-946^{d}$ & 3572 & $3572^{\mathrm{d}}$ & & \\
\hline (42) $\mathbf{C F}_{\mathbf{4}}$ & 29 & $594-1313$ & $582-1519$ & 60033 & 842709 & & \\
\hline (43) $\mathbf{C}_{\mathbf{4}} \mathbf{H}_{2}$ & 2211 & $0-758$ & $0-1303$ & 124126 & 251245 & & \\
\hline (44) $\mathbf{H C}_{3} \mathbf{N}$ & 1224 & $0-760$ & $0-760$ & 180332 & 180332 & & \\
\hline \multirow[t]{2}{*}{ (45) $\mathbf{H}_{2}$} & 11 & $15-36,024$ & $15-27,185$ & 4017 & 3480 & Yes & \\
\hline & $12^{\mathrm{b}}$ & $3-36,406$ & $3-36,406$ & 5129 & 5129 & Yes & \\
\hline \multirow[t]{4}{*}{ (46) CS } & 22 & $1-2586$ & $1-2586$ & 1088 & 1088 & & \\
\hline & 24 & $1-1359$ & $1-1359$ & 396 & 396 & & \\
\hline & 32 & $1-1331$ & $1-1331$ & 396 & 396 & & \\
\hline & 23 & $1-156$ & $1-156$ & 198 & 198 & & \\
\hline (47) $\mathrm{SO}_{3}$ & 26 & $0-2825$ & $0-2825$ & 10881 & 14295 & & \\
\hline (48) $\mathrm{C}_{2} \mathrm{~N}_{2}$ & 4224 & c & $200-307$ & c & 71775 & & \\
\hline (49) $\mathbf{C O C l}_{\mathbf{2}}$ & 2655 & c & $793-900$ & c & 164436 & & \\
\hline & 2657 & c & $800-892$ & c & 145477 & & \\
\hline
\end{tabular}

a Abbreviated code for isotopologues.

b Although spectral ranges and amount of lines is unchanged with respect to HITRAN2012, there are changes to spectral parameters of lines for these isotopologues.

c Not included in HITRAN2012.

d These numbers will change eventually once changes discussed in sections 2.8 and 2.41 will be finalized.

agreement with these intensities. The present update relies heavily on Lodi-Tennyson style calculations for all isotopologues, supplemented with high-quality experimental data where available. This approach has proven to work well in application to atmospheric and laboratory spectra (see for instance Campargue et al. [29] and Ponomarev et al. [30]).

Given the reliance on these theoretical intensities, it is important to understand the systematic errors which $a b$ initio calcu- lations may show. In the same issue of this journal, Birk et al. [31] give an extensive intercomparison of $a b$ initio calculations with high-quality experimental data. The experimental intensities used in the intercomparison are all included in the HITRAN2016 update. Much of the data show agreement between $a b$ initio and experiment within $2 \%$. However, for some bands, notably those involving excitation of the stretching modes, there are larger offsets of up to $8 \%$ which can be attributed to systematic errors in the $a b$ 

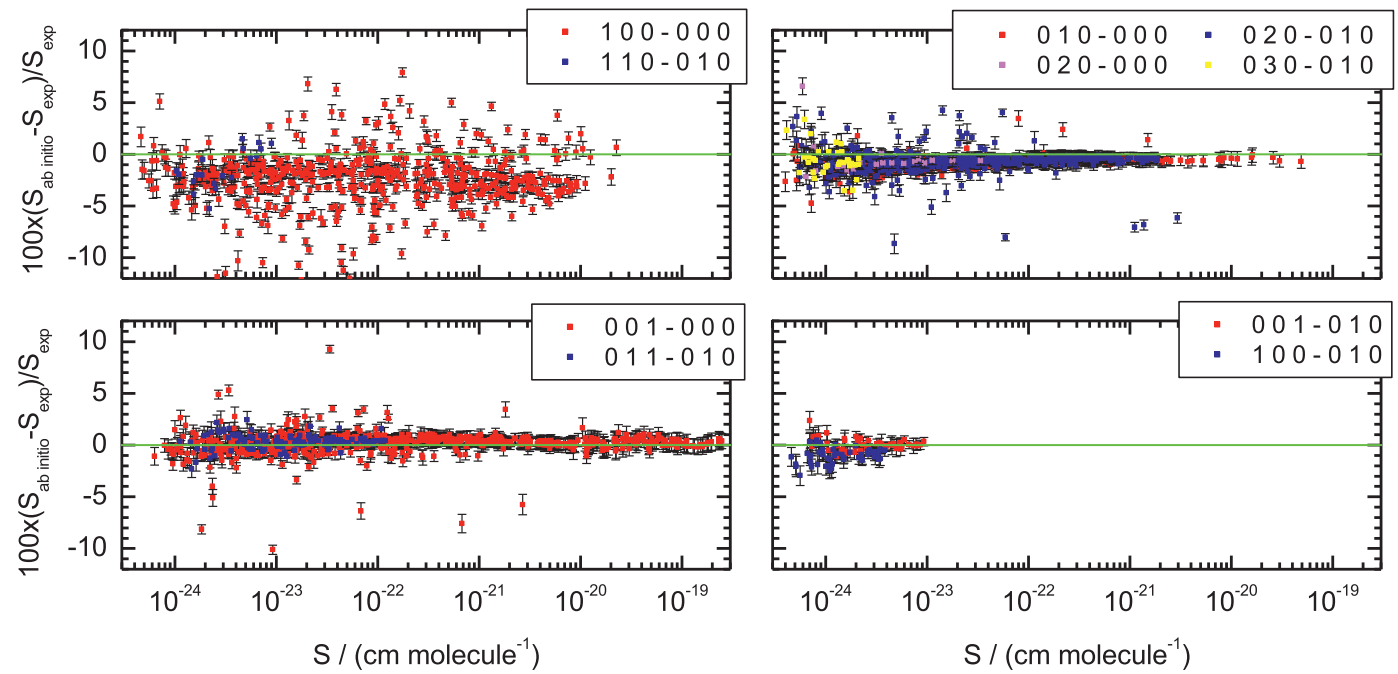

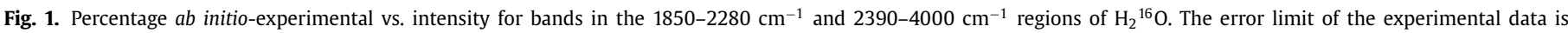
$1 \%[31]$.

initio dipole moment calculations. Furthermore, in the $v_{1}$ fundamental, differences show a systematic dependence on wavenumber, $\Delta K_{a}$ and $\Delta J$. Other vibrational bands involving the changes in $v_{1}$ quanta also showed larger systematic errors. The theoretical calculations also have problems in predicting local resonances accurately, although these resonances start to appear more frequently in the NIR part of the spectrum. The information on systematic differences between the ab initio theory and experiment for $\mathrm{H}_{2}{ }^{16} \mathrm{O}$ can be used to estimate uncertainties for minor isotopologues for which no high-accuracy experimental intensities are available.

An important finding of this work is that for an individual vibrational band an intensity-independent offset appears in graphical representations of differences between experiment and theory vs. intensity. This information can be used to validate experiment as well as theory. The constant offset can also be used to predict weak line intensities where experimental data are unavailable by scaling $a b$ initio values.

Fig. 1 compares theory and experiment for the $1850-2280 \mathrm{~cm}^{-1}$ and $2390-4000 \mathrm{~cm}^{-1}$ regions. The agreements for the fundamental bands (001)-(000), (010)-(000), the overtone (020)-(000), the hot bands (030)-(010), (020)-(010), (011)-(010), (001-010), (100)-(010) are all excellent, mainly within $2 \%$. The bands involving changes in the $v_{1}$ quanta, (100)-(000) and (110)-(010), however, show large scatter and an offset around $-2 \%$. The scatter includes the systematic differences of $+5 \%$ to $-13 \%$, which becomes obvious when plotting the differences against wavenumber or lower-state energy and color coding $\Delta K_{\mathrm{a}}$ and/or $\Delta J$ [31].

The 2016 update provides comprehensive line lists for the six main isotopologues of water: $\mathrm{H}_{2}{ }^{16} \mathrm{O}, \mathrm{H}_{2}{ }^{18} \mathrm{O}, \mathrm{H}_{2}{ }^{17} \mathrm{O}, \mathrm{HD}^{16} \mathrm{O}, \mathrm{HD}^{18} \mathrm{O}$ and $\mathrm{HD}^{17} \mathrm{O}$, as well as the newly-added isotopologue $\mathrm{D}_{2}{ }^{16} \mathrm{O}$. The use of variational calculations to provide the underlying line lists guarantees the completeness of the lists for the intensity cutoff employed. This completeness leads to a significant expansion of the number of important transitions of the deuterated isotopologues into the NIR (near-infrared) region. These line lists are presented and analyzed in articles published in this issue [25]. Fig. 2 shows an example where atmospheric retrieval from a Total Carbon Column Observing Network (TCCON) [32] spectrum benefits from inclusion of HDO lines in the NIR. It is also important to note a significant increase in coverage for $\mathrm{HD}^{17} \mathrm{O}$ and $\mathrm{HD}^{18} \mathrm{O}$ which were poorly represented in previous HITRAN releases.

The variational (calculated) intensities described above were then replaced with intensities from available high-quality experi- ments, many of which were already in the HITRAN2012 database. The details will be given in a dedicated paper, but some of the experiments that represent a substantial bulk of new experimental intensity data are described below.

2.1.1.1. DLR experiments. An extensive work in the spectral ranges $1850-2280 \mathrm{~cm}^{-1}$ and $2390-4000 \mathrm{~cm}^{-1}$ was carried out by Loos et al. [33,34] and in the range $4190-4340 \mathrm{~cm}^{-1}$ by Birk et al. [31]. In Fig. 3 we will refer to these, and the experiments from the same authors that are already in HITRAN, as DLR. In Loos et al. [33,34] line positions, intensities, self- and air-broadened line-shape parameters, their temperature dependence as well as Rosenkranz line coupling coefficients were retrieved from numerous Fourier-Transform transmittance measurements of self- and air-broadened water vapor at $296 \mathrm{~K}$ as well as air-broadened water vapor measurements at low and high temperatures. During the analysis, a large effort was undertaken to give consolidated error bars. In the analysis, a multispectrum fitting approach applying a quadratic speed-dependent hard collision model based on the Hartmann-Tran profile $[7,8]$ and extended to account for line mixing in the Rosenkranz first order perturbation approximation [35] was used. Line positions, intensities and self-broadening, self-speed-dependence and self-shift parameters, as well as in some cases self-line coupling coefficients, were retrieved from pure water-vapor measurements of total pressures up to 20 mbar. Airbroadening, speed-dependence, pressure shift parameters, Dicke narrowing and line-mixing coefficients as well as temperaturedependence parameters were obtained from air-broadened measurements at ambient temperature and total pressures from 30 to $1000 \mathrm{mbar}$ as well as low and high-temperature measurements at $100 \mathrm{mbar}$ total pressure. The intensities of lines with retrieved line parameters range from $3 \times 10^{-26}$ to $3 \times 10^{-19} \mathrm{~cm}^{-1} /$ (molecule $\mathrm{cm}^{-2}$ ). In the $4190-4340 \mathrm{~cm}^{-1}$ region, several Fourier-Transform transmittance spectra of pure and air-broadened water vapor at $296 \mathrm{~K}$ as well as low and high temperatures were measured and analyzed by Birk et al. [31]. These measurements were dedicated to water vapor parameters to be used in TROPOMI/S5-P [36] retrievals. The analysis was also based on a multispectrum fit using the HT profile. Line positions, intensities, self- and air-broadened line-shape parameters including speed-dependence and Dicke narrowing parameters as well as their temperature dependence were retrieved in the analysis. 

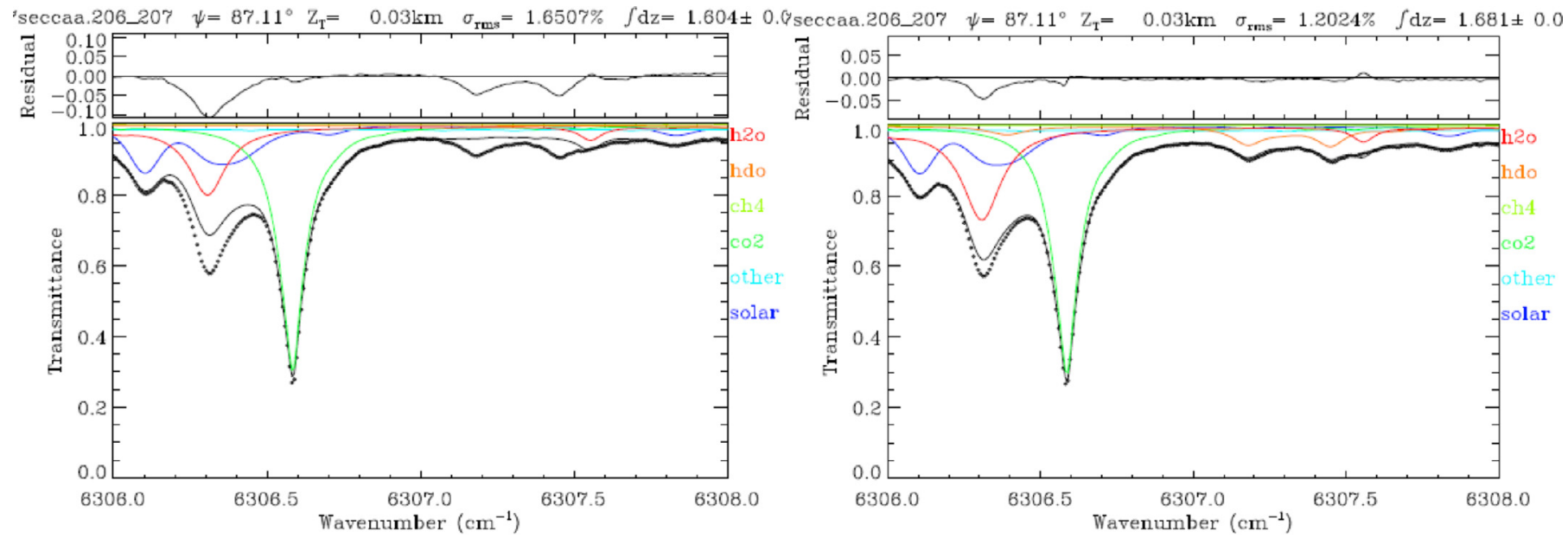

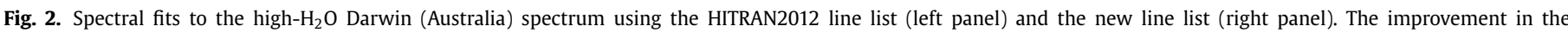
residuals is due to the addition of HDO lines (three in this plot) denoted by the orange trace. Note the slight change of scale in the residuals on the left and right sides.

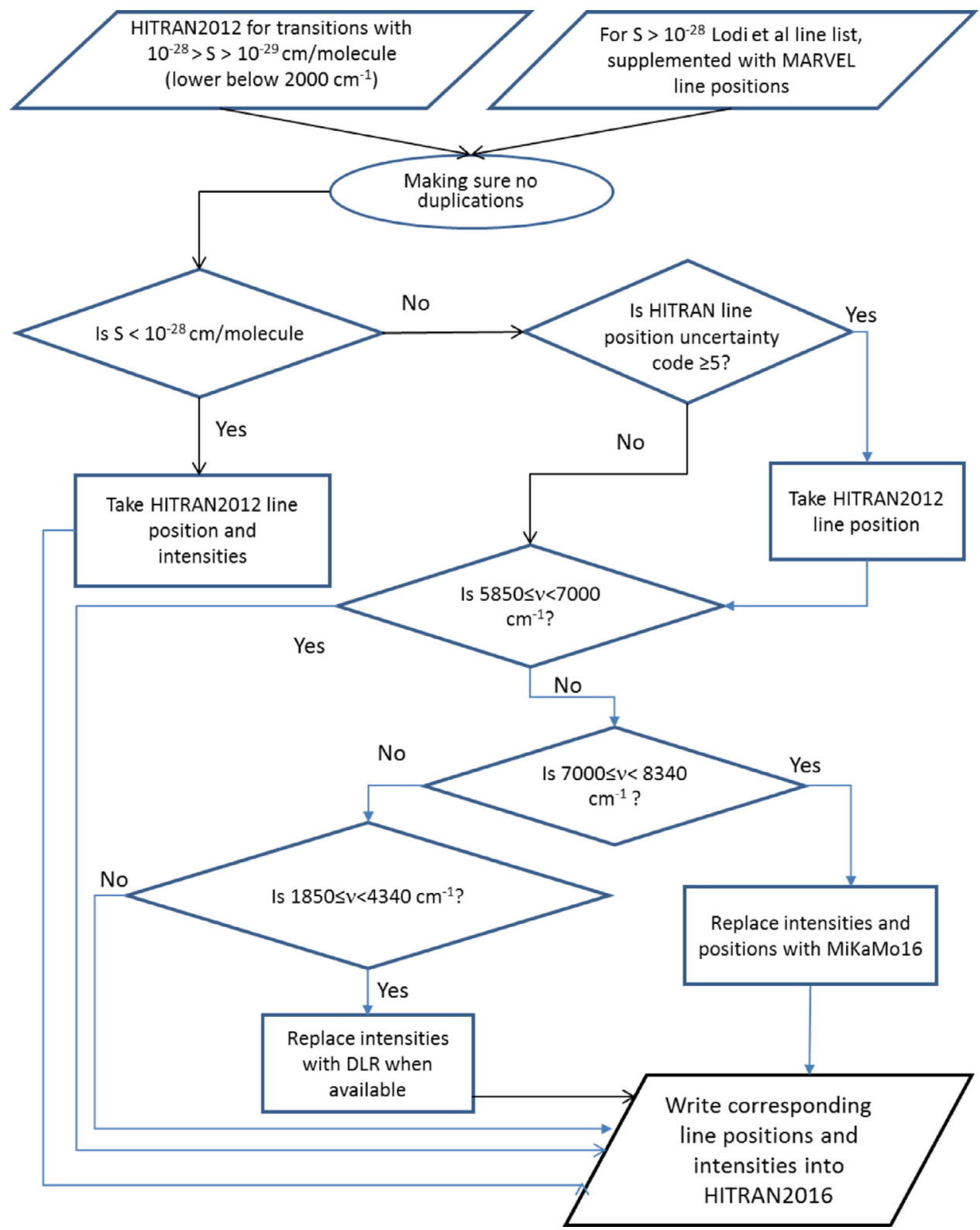

Fig. 3. Flow diagram for the construction of line positions and intensities for $\mathrm{H}_{2}{ }^{16} \mathrm{O}$ below $8340 \mathrm{~cm}^{-1}$. See text for details. 


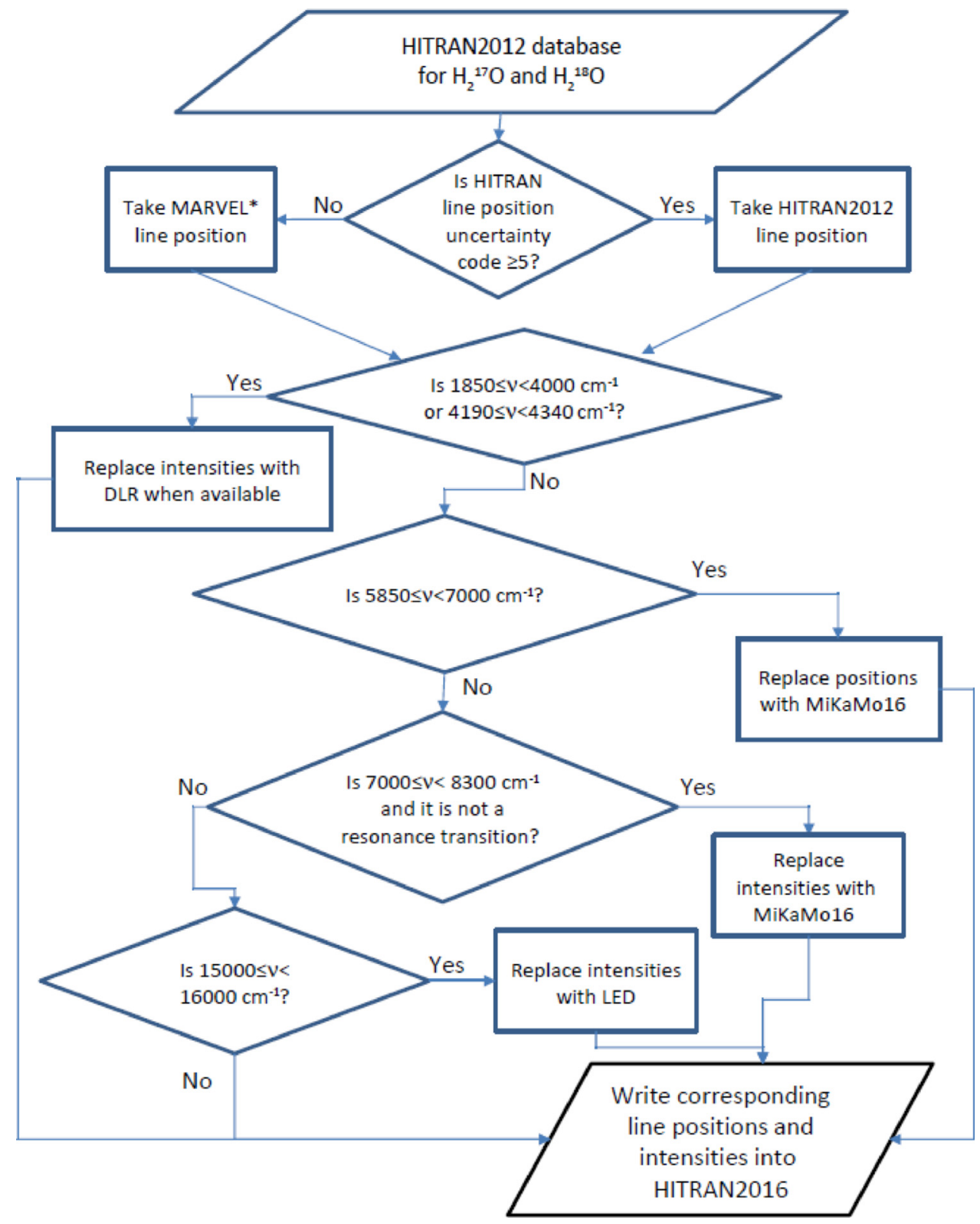

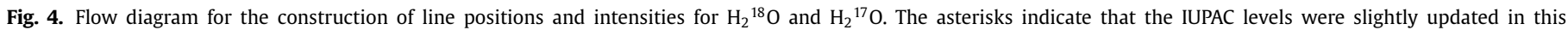
work. LED refers to the experimental data from Ref. [26]. See text for details.

2.1.1.2. MiKaMo16 experimental set. Recently, Mikhailenko et al. [23] have compiled a database of measurements in 5850$8340 \mathrm{~cm}^{-1}$ region from the Grenoble-Tomsk collaboration reported in Refs. [37-41] supplemented with measurements from Sironneau and Hodges [42] and for strong lines from Toth [43] and for very weak lines from ab initio work of Partridge and Schwenke $[44,45]$. This database is here referred to as MiKaMo16. Unfortunately, due to some differences in assignments with the variational Lodi-Tennyson-like line lists described above, it was hard to take full advantage of this line list. However, tests against TCCON spectra have shown that this line list is superior to any other line list in the region of $7000-8340 \mathrm{~cm}^{-1}$. It was therefore used in HITRAN2016 in that region as is almost everywhere in this spectral region. This, however, creates some consistency issues with assignments of energy levels throughout the database, but substantial improvement of atmospheric retrievals justifies this approach.
As examples of the complexity of the update, Figs. 3 and 4 show the flow diagrams of the construction of position-intensity line lists of the principal isotopologue (only up to $8340 \mathrm{~cm}^{-1}$ for simplicity) and for $\mathrm{H}_{2}{ }^{18} \mathrm{O} / \mathrm{H}_{2}{ }^{17} \mathrm{O}$ respectively. Note that the line list from Lodi et al. [16] for the principal isotopologue is limited to $10^{-28} \mathrm{~cm}^{-1} /\left(\right.$ molecule $\left.\mathrm{cm}^{-2}\right)$; therefore intensities for weaker lines still originate from HITRAN2012/BT2.

Whenever a rotational quantum number could not be determined unambiguously, the index of symmetry $(1,2,3$, and 4 as defined in the BT2 work [15]) accompanied with a negative sign was used. Note that 1 and 2 indicate para states, whereas 3 and 4 indicate ortho states. For the case of unassigned vibrational quanta, a "-2" label has been adopted. 


\subsubsection{Line-shape parameters for the $\mathrm{H}_{2}{ }^{16} \mathrm{O}, \mathrm{H}_{2}{ }^{18} \mathrm{O}$ and $\mathrm{H}_{2}{ }^{17} \mathrm{O}$ line} lists

The air-broadened half widths (in Voigt profile formalism) for the first three isotopologues of water in HITRAN2008 and, with some modifications, in HITRAN2012 were derived using the procedure "Diet" described by Gordon et al. [46]. This procedure continues to be used in this edition with experimental outliers identified and removed. One notable example is the removal of a large portion of the half widths measured in Jenouvrier et al. [47] from the experimental data that feeds the "Diet". Indeed, some of the recent evaluations of atmospheric spectra showed that many data in Jenovrier et al. are questionable (see for instance Armante et al. [48]). However, although the removal of this extensive dataset from the updated Gordon et al. [46] procedure, as was done in the GEISA database [49] and the MiKaMo16 linelist, have improved the residuals in some of the lines, some of the residuals became worse than in HITRAN2012. The main problem here is due to the fact that, while there are definite outliers in the values reported in Jenouvrier et al. [47], it is the most extensive study of the air-broadened line widths in the $4200-6600 \mathrm{~cm}^{-1}$ region. In fact, for many transitions no other measurements exist. Removal of these measurements from the experimental dataset has invoked the use of the approximations of different levels (some are very crude) from the work of Jacquemart et al. [50] for many of the transitions. The only solution in this case was to filter the Jenouvrier et al. dataset. This was done through direct comparisons with the TCCON spectra for some of the transitions and cross-comparison with similar rotational transitions but from other bands, accounting for vibrational dependence from Eq. (1) of Jacquemart et al. [50]. After this filtering procedure, about 1400 measurements (out of almost 5000) from Jenouvrier et al. [47] were retained.

It is also important to emphasize that measurements by Birk and Wagner [51] in the $v_{2}$ band region were given a priority and were written into the database directly as was done in $\mathrm{HI}-$ TRAN2012.

Since the modeling of water vapor absorption lines using a Voigt model is no longer sufficient in many applications and the HT profile provides considerably higher accuracy, experimental HT profile parameters of Birk et al. and Loos et al. [31,33,34] in the spectral ranges $1850-2280 \mathrm{~cm}^{-1}, 2390-4000 \mathrm{~cm}^{-1}$ and $4190-$ $4340 \mathrm{~cm}^{-1}$ were accommodated wherever possible. The experiments and analysis procedures applied are described briefly in Section 2.1.1.

\subsubsection{Line-shape parameters for the deuterated isotopologues}

The addition of the line-shape parameters for $\mathrm{D}_{2} \mathrm{O}$ follows a similar procedure as the other isotopologues; however, the available data for air-broadening of $\mathrm{D}_{2} \mathrm{O}$ are considerably less complete than for the other isotopologues. There are some measurements for air-broadening of $\mathrm{D}_{2} \mathrm{O}$ [52-55] as well as Complex Robert-Bonamy calculations [56]. These data amount to several hundred lines from the measured data and just over 550 lines from the theoretical calculations. Using these data, average values as a function of $J^{\prime \prime}$ were determined and extrapolation to $J^{\prime \prime}=50$ was done using a thirdorder polynomial fit.

Using these data, the half widths and some line shifts have been added to the $\mathrm{D}_{2} \mathrm{O}$ lines in the database using a priority scheme. First, the measured values were added; if the measurement did not exist for the transition in question, the theoretical value was used. If neither exists, the $J$-averaged value was used. This algorithm allowed half widths to be added to all $\mathrm{D}_{2} \mathrm{O}$ lines in the HITRAN database.

For self-broadening parameters of $\mathrm{D}_{2} \mathrm{O}$, the work of Gamache et al. [57] was used. These data are an extensive set of calculations for $\mathrm{D}_{2} \mathrm{O}$ rotational band transitions extended to 6536 lines using the partner transition rule [58]. Using these data, a set of $J$-averaged half widths was determined for $J=0-50$ [57]. These data have been added to the algorithm that adds line-shape data to the water lines in the HITRAN database.

\subsubsection{Future plans}

Recently, Lampel et al. [59] have identified important absorption features in atmospheric spectra due to water vibrationrotation transitions in the near ultraviolet around $363 \mathrm{~nm}$. Laboratory measurements of water vibration-rotation transition intensities only extend to $25,470 \mathrm{~cm}^{-1}(393 \mathrm{~nm})$ [60] although multiphoton spectra do provide some information on energy levels in the region of question [61,62]. Future updates will look to extend coverage to water absorption in the near ultraviolet; these data are important for upcoming satellite missions, including TEMPO [63] which will record spectra at these wavelengths.

At the intensity limit assumed for $\mathrm{D}_{2}{ }^{16} \mathrm{O}$, the even rarer isotopologues $\mathrm{D}_{2}{ }^{17} \mathrm{O}$ and $\mathrm{D}_{2}{ }^{18} \mathrm{O}$ should also be visible. Line lists for these two species have also been prepared as part of the work on deuterated water in Ref. [25]; these data will be added in the forthcoming updates to HITRAN2016.

We will be populating the database of the HT line-shape parameters after evaluating existing literature values and new measurements as they become available.

\section{2. $\mathrm{CO}_{2}$ (molecule 2)}

Accurate and comprehensive line lists for all naturally abundant isotopologues of carbon dioxide are required by remote-sensing missions dedicated to monitor the concentration of carbon dioxide in Earth's atmosphere. The recently launched OCO-2 mission [64$66]$, together with several other space and ground based projects (GOSAT [67], AIRS [68], ASCENDS [69], TCCON [32], NDACC [70]) are dedicated to explicitly monitor the atmospheric $\mathrm{CO}_{2}$ content. These experiments aim not only to look at overall $\mathrm{CO}_{2}$ concentration and its variation, but also wish to pinpoint where $\mathrm{CO}_{2}$ is being produced (sources) and where it is absorbed (sinks). This activity is clearly vital to monitoring and essential for eventually controlling the $\mathrm{CO}_{2}$ content of the atmosphere [71]. A successful retrieval of $\mathrm{CO}_{2}$ concentration requires validated line lists with transition intensities given at sub-percent accuracy, line positions accurate to $0.0001 \mathrm{~cm}^{-1}$ or better, and beyond-Voigt-profile line-shape models $[65,72,73]$.

Determination of isotopic ratios of carbon in Earth's samples and astrophysical objects remains crucial for modeling geophysical processes. For example, quantification of ${ }^{14} \mathrm{C}$ in fossil fuels can provide information about the sources of human-related contribution to the total $\mathrm{CO}_{2}$ concentration in the terrestrial atmosphere. This can be done with recently developed cavity-enhanced laser spectroscopic techniques in the IR [74-76]. However, these measurements require a priori simultaneous knowledge of reliable line intensities of many isotopologues. Precise determination of ${ }^{13} \mathrm{C} /{ }^{12} \mathrm{C}$ and ${ }^{16} \mathrm{O} /{ }^{17} \mathrm{O} /{ }^{18} \mathrm{O}$ ratios is also vital, for instance, in understanding processes of formation of radiation fields in the Martian atmosphere, which is $96 \%$ rich in carbon dioxide [77].

A summary of the carbon dioxide line list in the HITRAN2012 database and comparison to HITRAN2016 is given in Table 4. The HITRAN2012 database was considerably improved with respect to its previous 2008 edition. However, several issues related to spectral completeness, inconsistency of multiple data sources, and insufficient accuracy of line intensities, still remained unsolved. The majority of entries in the 2012 version of the HITRAN database were taken from the effective Hamiltonian calculations included in the 2008 edition of the CDSD-296 database [78].

For less abundant isotopologues, obtaining high-quality experimental data is not trivial. Therefore fits of the effective Hamiltonian or the effective dipole moment [78], were based on only four 
Table 4

Comparison of HITRAN2016 and HITRAN2012 line lists for isotopologues of carbon dioxide.

\begin{tabular}{|c|c|c|c|c|c|c|}
\hline \multirow[t]{2}{*}{ ISO/abundance } & \multicolumn{3}{|l|}{ HITRAN2012 } & \multicolumn{3}{|l|}{ HITRAN2016 } \\
\hline & Number of lines & Spectral region $\left(\mathrm{cm}^{-1}\right)$ & $\mathrm{Q}(296 \mathrm{~K})$ & Number of lines & Spectral region $\left(\mathrm{cm}^{-1}\right)$ & $Q(296 \mathrm{~K})$ \\
\hline $626 / 0.984204$ & 169,292 & $345.936-12,784.056$ & 286.94 & 173,024 & $158.301-14,075.298$ & 286.094 \\
\hline $636 / 1.1057 \times 10^{-2}$ & 70,611 & $406.834-12,462.046$ & 578.41 & 70,577 & $332.649-13,734.963$ & 576.644 \\
\hline $628 / 3.9470 \times 10^{-3}$ & 116,482 & $0.736-9557.398$ & 609.48 & 127,850 & $1.472-12,677.181$ & 607.713 \\
\hline $627 / 7.3399 \times 10^{-4}$ & 72,525 & $0.757-9599.317$ & 3552.70 & 77,941 & $0.757-12,726.562$ & 3542.610 \\
\hline $638 / 4.4345 \times 10^{-5}$ & 26,737 & 489.678-6744.158 & 1229.10 & 43,782 & $2.945-9212.609$ & 1225.270 \\
\hline $637 / 8.2462 \times 10^{-6}$ & 2953 & $583.593-6768.643$ & 7162.90 & 25,175 & $9.086-8061.741$ & 7140.024 \\
\hline $828 / 3.9556 \times 10^{-6}$ & 7118 & $491.688-8160.439$ & 324.21 & 10,522 & $482.813-8162.743$ & 323.424 \\
\hline $728 / 1.4718 \times 10^{-6}$ & 821 & $626.438-5046.875$ & 3776.40 & 15,878 & 491.181-8193.172 & 3766.044 \\
\hline $727 / 1.3685 \times 10^{-7}$ & 5187 & $535.384-6932.980$ & $11,002.00$ & 6518 & $535.383-6932.693$ & $10,971.91$ \\
\hline $838 / 4.4440 \times 10^{-8}$ & 121 & 4599.239-4887.290 & 653.50 & 2916 & $2245.898-4750.068$ & 652.242 \\
\hline $837 / 1.653 \times 10^{-8}$ & $\mathrm{~N} / \mathrm{A}$ & $\mathrm{N} / \mathrm{A}$ & 7615.20 & 4190 & $549.472-4914.496$ & 7593.900 \\
\hline $737 / 1.5375 \times 10^{-9}$ & $\mathrm{~N} / \mathrm{A}$ & $\mathrm{N} / \mathrm{A}$ & $\mathrm{N} / \mathrm{A}$ & 1501 & $575.852-3614.084$ & $22,129.96$ \\
\hline $646 /$ radioactive & $\mathrm{N} / \mathrm{A}$ & $\mathrm{N} / \mathrm{A}$ & $\mathrm{N} / \mathrm{A}$ & 41,610 & $426.445-7928.788$ & 2033.353 \\
\hline
\end{tabular}

Note: ISO is the AFGL shorthand notation for the isotopologue, abundance is the terrestrial value assumed by HITRAN, and Q(296) is the partition sum at $296 \mathrm{~K}$.
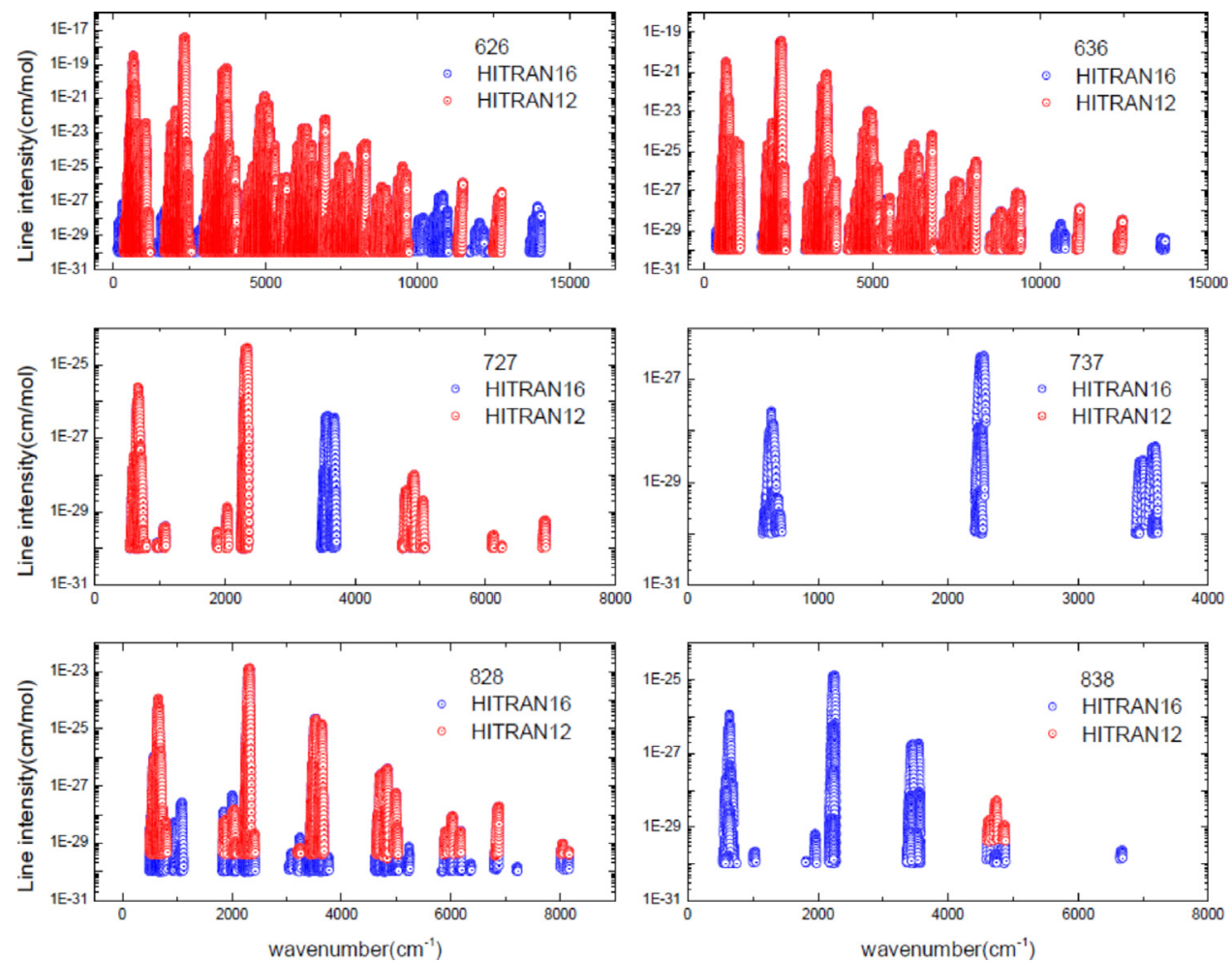

Fig. 5. Overview of the line lists of stable symmetric isotopologues of carbon dioxide in HITRAN2012 and HITRAN2016.

major isotopologues ${ }^{12} \mathrm{C}^{16} \mathrm{O}_{2},{ }^{13} \mathrm{C}^{16} \mathrm{O}_{2},{ }^{16} \mathrm{O}^{12} \mathrm{C}^{18} \mathrm{O}$ and ${ }^{16} \mathrm{O}^{12} \mathrm{C}^{17} \mathrm{O}$, for which measured spectroscopic parameters were available. As a result, several spectral gaps were present in HITRAN2012 (see for instance discussion in Refs. $[79,80])$ which represent regions where experimental data were unavailable. For similar reasons, no entries were included in the database for the ${ }^{18} \mathrm{O}^{13} \mathrm{C}^{17} \mathrm{O},{ }^{17} \mathrm{O}^{13} \mathrm{C}^{17} \mathrm{O}$, and ${ }^{16} \mathrm{O}^{14} \mathrm{C}^{16} \mathrm{O}$ isotopologues (837, 737, and 646 in old AFGL notation). An overview of this problem is displayed in Figs. 5 and 6, where $\mathrm{CO}_{2}$ ro-vibrational spectra from HITRAN2012 and HITRAN2016 are compared for different isotopologues.

Wherever possible, the effective Hamiltonian fits were extrapolated to the trace isotopologues, using a method of isotopic substitution [81]. In the 2012 edition, multiple data sources caused sporadic discontinuities in intensity patterns of ro-vibrational lines [82-84]. Furthermore, a high percentage of line intensities in HITRAN2012 have stated uncertainty of $20 \%$ or worse (HITRAN uncertainty index equal to 3 ). Although this assessment has been proven to be overly pessimistic in many cases [82,83,85-87], the uncertainty budget, especially for the Effective Hamiltonian calculations, was still too high for precise measurements of atmospheric $\mathrm{CO}_{2}$ concentration. The most accurate entries in HITRAN2012 were taken from NASA JPL measurements by Toth et al. [88-90] and covered the $1.6-\mu \mathrm{m}$ and $2.06-\mu \mathrm{m}$ spectral regions, which are used in remote-sensing measurements. The stated 1$5 \%$ accuracy of these experimental line intensities (HITRAN uncertainty index equal to 7 and 6), has been confirmed by a number of comparisons [82,83,91]; nonetheless the rigorous requirements for part-per-million resolution in measurements of $\mathrm{CO}_{2}$ atmospheric concentration were not achieved.

Since the 2012 release of the HITRAN database, a large number of experimental and theoretical studies have been devoted to improve the knowledge of line positions, line intensities and line shapes of $\mathrm{CO}_{2}$ isotopologues. For a comprehensive review of 

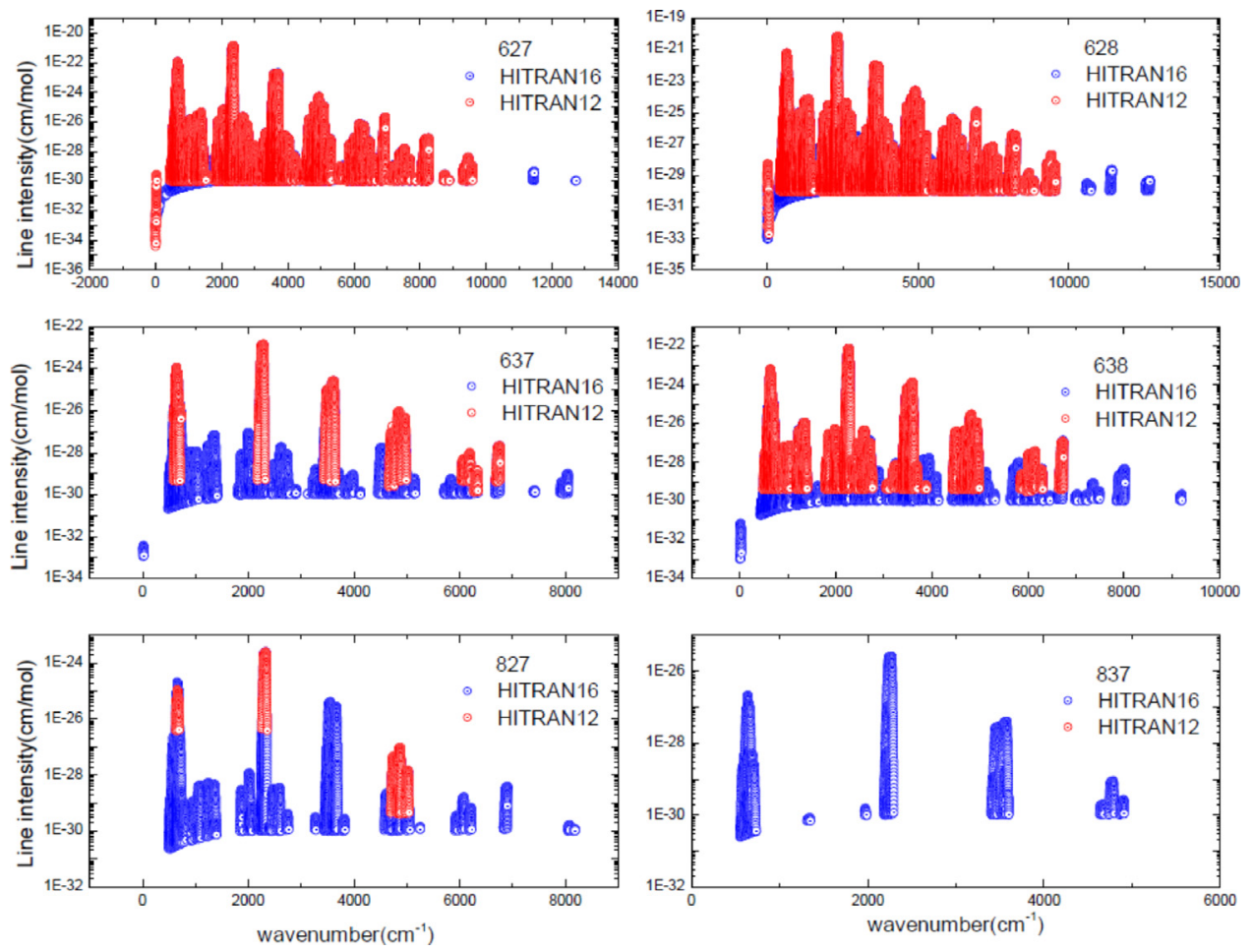

Fig. 6. Overview of the line lists of stable asymmetric isotopologues of carbon dioxide in HITRAN2012 and HITRAN2016.

measurements and theoretical models see Ref [92]. and references therein.

Theoretical line lists (denoted as "Ames") for 12 stable and one radioactive (646) isotopologue of $\mathrm{CO}_{2}$ were published by Huang et al. in the $0-20,000 \mathrm{~cm}^{-1}$ spectral region and for temperatures below $1500 \mathrm{~K}[79,93,94]$. Room-temperature line lists (denoted as "UCL-IAO") for $13 \mathrm{CO}_{2}$ isotopologues were also calculated by Zak et al. $[82,83,85,91]$ in the $0-8000 \mathrm{~cm}^{-1}$ spectral region. Both of these latter studies contained intensities computed with $a b$ initio dipole moment surfaces and semi-empirical line positions, based on a fitted potential energy surface for the Ames effort and on the effective Hamiltonian calculations for UCL-IAO. A major advantage of the variational approach used in the Ames and UCL-IAO line lists is that it should give similar accuracy for all isotopologues. This allows coverage of spectral regions currently not probed by experiments for rare isotopologues. UCL-IAO also provides uncertainty estimates of line intensities, based on a purely theoretical methodology [17]. Such a reliable analysis allows for the detection of ro-vibrational resonance interactions, which significantly lower the accuracy of theoretical line positions and intensities. Using this method, the lines identified as unreliable have been replaced with the data from CDSD-296 [92] and, in several cases of interpolyad resonance interactions (asymmetric isotopologues), with the experimental data from Lyulin et al. [95], Karlovets et al. [96,97] and Campargue et al. [98].

Recently, there have been a number of high-precision near-IR spectroscopic measurements which provide rigorous tests of theoretical line intensities based on effective dipole moment surface and ab initio calculations [84-87,99]. Particularly, in the $1.6-\mu \mathrm{m}$ and $2.0-\mu \mathrm{m}$ spectral regions, the UCL-IAO line lists have been experimentally verified as accurate to the sub-percent level. Fig. 7 compares the UCL-IAO and Ames line lists to HITRAN2012 (Toth et al. blue open circles [100, 101]) for the $20012-00001$ band and to state-of-the-art experiments including advanced high-resolution laser measurements [101-104]. A number of comparisons here suggest that the UCL-IAO study models line intensities more ac-

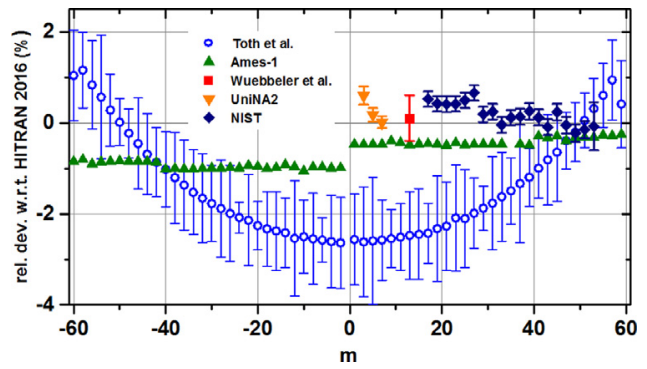

Fig. 7. Comparison of line intensities between HITRAN2016, HITRAN2012 (Toth et al. [88]), and other accurate experimental and theoretical sources for the 20012 00001 band (2- $\mu \mathrm{m}$ band) of ${ }^{12} \mathrm{C}^{16} \mathrm{O}_{2}$ : measurements Wübbeler et al. [102], NIST [104] and UniNA2 [103], and theory Ames-1 [79]. The zero relative deviation line corresponds to HITRAN2016 line intensities (in this case from Zak et al. [82]). The running index $m$ equals $-J, J$, and $J+1$ for the $\mathrm{P}, \mathrm{Q}$, and $\mathrm{R}$ branches, respectively.

curately than the Ames study. Note that more recent results from Ames, which are available from their website (www.huang.seti. org), give closer agreement with UCL-IAO. From Fig. 7 it is clear that there is a $1-3 \%$ average difference in line intensities between the new and the previous version of HITRAN for this band. The independent experiments from the National Institute of Standards and Technology [104], and the University of Naples II [103] confirm, however, a conservative $0.5 \%$ accuracy of line intensities for this band in HITRAN2016. This level of accuracy could potentially satisfy even the most stringent requirements of current remotesensing missions. Interestingly, although line intensities for this band and the 20013 - 00001 and 30013 - 00001 bands probed by OCO- 2 originate from the same source (Toth et al. [100]), the agreement between UCL-IAO and HITRAN2012 is substantially better for the OCO-2 bands.

For wavenumbers greater than $8000 \mathrm{~cm}^{-1}$, the majority of the line parameters were taken from CDSD-296 [92]. As we have already mentioned above, both HITRAN2012 and CDSD-296 have several spectral gaps, in particular in the wavenumber region 
greater than $8000 \mathrm{~cm}^{-1}$. Recently, several experimental studies of the carbon dioxide spectra in the high-frequency region have been performed [105-108]. The measured line intensities allowed determining the absent effective dipole moment parameters for several series of transitions. Using these effective dipole moment parameters and an effective Hamiltonian from Ref. [109], the line positions and intensities for the principal isotopologue were generated and included into HITRAN2016 covering the $9800-10,500 \mathrm{~cm}^{-1}$ and $11,600-12,400 \mathrm{~cm}^{-1}$ wavenumber gaps.

Line positions were updated with respect to the previous version of the database. The majority of lines come from the effective Hamiltonian calculations included in UCL-IAO line lists, which are based on the fits to the observed line positions collected from the literature and published in the latest, 2015 release of the CDSD296 database [92]. These fits were completed and updated with recent, accurate measurements performed on isotopically-enriched samples of $\mathrm{CO}_{2}$. Uncertainties in the fitted line positions depend on the quality of the experimental data and vary from $0.001 \mathrm{~cm}^{-1}$ to $10^{-9} \mathrm{~cm}^{-1}$. For asymmetric isotopologues, a number of bands are affected by strong interpolyad anharmonic resonance interactions. The effective Hamiltonian model does not include this type of interaction for the asymmetric isotopologues. Hence in such cases, line positions were taken directly from measurements [80,95-98,110,111].

The uncertainty codes for the line positions were transferred from CDSD-296 to HITRAN2016. The uncertainty code 3 (0.001-0.01 $\left.\mathrm{cm}^{-1}\right)$ was given for the line positions in the 9800$10,500 \mathrm{~cm}^{-1}$ and $11,600-12,400 \mathrm{~cm}^{-1}$ wavenumber regions. Partition functions in the current release of the database are based on the direct summations taken from the variational calculations of Huang et al. [79]. On average, the new partition functions agree excellently with those of CDSD-296 [83]; however they do not agree perfectly with those in HITRAN2012 (from TIPS [112]) and three previous editions of the database and differ at $296 \mathrm{~K}$ by about $-0.3 \%$. Although this difference is marginal, it could have an effect in the applications where sub-percent accuracy is required.

As stated above, the radioactive isotopologue ${ }^{14} \mathrm{CO}_{2}, 646$, has been added to the database. This is the first edition of HITRAN where radioactive species have been incorporated (also for $\mathrm{CO}$, see Section 2.5). All lines of the 646 isotopologue were taken from the UCL line lists given in Ref. [83]. Due to issues with what constitutes a so-called natural terrestrial abundance of radioactive species (which is part of the traditional definition of intensities in HITRAN, see Eq. (1) of the Definitions and Units documentation in HITRANonline), line intensities for these type of species are given for unit abundance; a $10^{-27} \mathrm{~cm}^{-1} /\left(\right.$ molecule. $\left.\mathrm{cm}^{-2}\right)$ cut-off value for the intensity has been applied. This cut-off produced 41,610 lines in the $J$ range 0 to 114 . Vibrational assignments for the 646 isotopologue were based on isotopic shifts of energy levels and respective assignments for the 626 and 636 isotopologues, and hence should be regarded as provisional. An abundance-scaled intensity cut-off of $10^{-30} \mathrm{~cm}^{-1} /\left(\right.$ molecule $\left.\cdot \mathrm{cm}^{-2}\right)$ is used for all stable isotopologues. Note that, for the time being, data for the radioactive isotopologues are provided as static files rather than through the HITRANonline interface.

Uncertainties of line intensities were informed by theoretical error analysis, which classified lines as reliable, intermediate, or unreliable. Bands with reliable lines stronger than $10^{-23} \mathrm{~cm}^{-1} /\left(\right.$ molecule $\cdot \mathrm{cm}^{-2}$ ) (for unit abundance) were assigned HITRAN uncertainty code 8 (i.e. accuracy of $1 \%$ or better). Line intensities of reliable parallel bands weaker than $10^{-23} \mathrm{~cm}^{-1} /\left(\right.$ molecule $\cdot \mathrm{cm}^{-2}$ ) were given an uncertainty code 7 (i.e. accuracy $1-2 \%$ ). Reliable perpendicular bands weaker than $10^{-23} \mathrm{~cm}^{-1} /\left(\right.$ molecule $\left.\cdot \mathrm{cm}^{-2}\right)$ and intermediate lines were marked with HITRAN uncertainty code 6 (i.e. accuracy 2-5\%). So-called un- reliable lines were taken from the effective dipole moment calculations [92] and experiments. Typical intensity uncertainties for these entries range between 5 and $20 \%$.

It is important to point out that an intensive study of the 1.6$\mu \mathrm{m}$ and $2.06-\mu \mathrm{m}$ bands that includes non-Voigt lineshapes and line mixing has been published by the OCO-2 spectroscopy support group ABSCO (ABSorption COefficient tables for the OCO-2 mission) $[84,86]$. The data were fit using a multi spectrum fit procedure which, among other things, enables retrieval of the line-shape parameters using the speed-dependent Voigt (SDV) profile as well as line mixing. These are very good experiments and it is debatable whether to use them for the strong and weak bands in place of UCL-IAO parameters described above. Indeed the ABSCO team have validated (using TCCON spectra) the cross-sections generated using results of Refs. [84,86] and found them to be the most efficient [113]. However, achieving high-precision results in nuanced correlations, with line mixing and model assumptions that can create discontinuities in inter-band comparisons, is difficult. At the moment, HITRAN cannot provide users with tools that can be used to generate cross-sections from the works of Devi et al. [84] and Benner et al. [86]. The usable products of the ABSCO effort are absorption coefficients (available upon request from the ABSCO group) rather than spectral parameters, which are available in the publications. Moreover, these absorption coefficients are empirically scaled by the factors of $0.6 \%$ and $1.4 \%$ for the $1.6-\mu \mathrm{m}$ and $2.06-\mu \mathrm{m}$ bands respectively, due to lingering data and/or model biases (the use of partition function HITRAN 2012/TIPS is up to $0.3 \%$ of this factor). After these studies, an update of the multi spectrum fitting code with CDSD partition functions was done. Additional methods to adjust the intensity distribution closer to the UCL list by scaling experimental conditions within the uncertainties are under evaluation. The intensities of the band at $2.06 \mu \mathrm{m}$ are already within $0.7 \%$ of Zak et al. [82], indicating that the additional $0.7 \%$ scaling of ABSCO cross-sections may be unrelated to intensities. These issues will be considered for future editions of the database.

For wavenumbers greater than $8000 \mathrm{~cm}^{-1}$, two sources of the line intensities are used: CDSD-296 [92] and the newly-generated line list in the $9800-10,500 \mathrm{~cm}^{-1}$ and $11,600-12,400 \mathrm{~cm}^{-1}$ regions based on the new measurements [105-108]. The uncertainty codes of the CDSD-296 line intensities were transferred to HITRAN2016. Based on the uncertainties of the line intensity measurements in the $10,700-10,860 \mathrm{~cm}^{-1}$ region [106], we use uncertainty code 5 $(5 \%-10 \%)$ for the line intensities of the $3003 \mathrm{i}-00001 \quad(i=1,2,3,4)$ series of bands and based on the uncertainties of the line intensity measurements in the $10,000-10,300 \mathrm{~cm}^{-1}$ and $11,600-$ $12,400 \mathrm{~cm}^{-1}$ [107] wavenumber regions we use uncertainty code $3(>20 \%)$ for the line intensities of the $4003 \mathrm{i}-00001(\mathrm{i}=1,2,3,4,5)$ and of the $6001 \mathrm{i}-00001(\mathrm{i}=1,2,3,4,5,6,7)$ series of bands.

The Voigt line-shape parameters throughout the entire database were calculated using the predictive routine of Gamache and Lamouroux explained in Refs. [114-116]. For the line mixing, we now provide a code from Lamouroux et al. [117] which has been updated to operate with HITRAN2016. We note that Lamouroux et al. [117] line mixing coupled with the HITRAN2012 data has worked really well and in fact produced residuals hardly exceeding $1 \%$ when applied to the TCCON data in Ref. [113], although slightly inferior to the ABSCO cross-sections in the $2.06-\mu \mathrm{m}$ region.

\section{3. $\mathrm{O}_{3}$ (molecule 3)}

The spectroscopic database for ozone is important for atmospheric and environmental applications due to its critical roles in UV shielding, radiative transfer and health. It is also considered a possible bio-marker for future observations of exoplanets [118]. In order to understand the impacts of ozone on the atmosphere and 


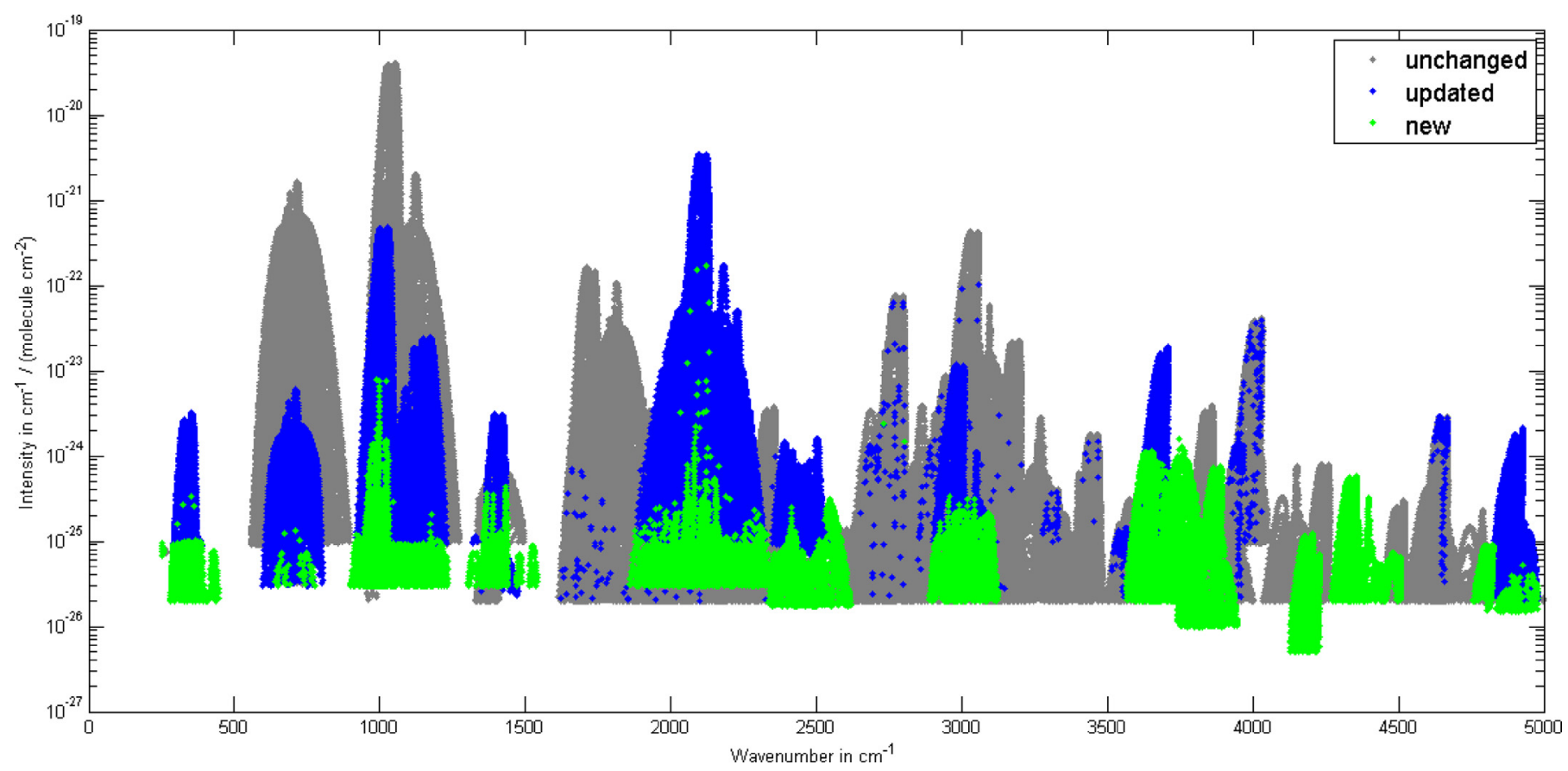

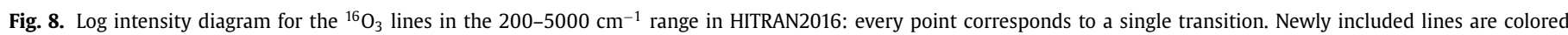
green, updated lines blue and unchanged HITRAN2012 lines grey (intensities are given in $\mathrm{cm}^{-1} / \mathrm{molecule}^{-\mathrm{cm}^{-2}}$ at $296 \mathrm{~K}$ ).

to monitor ozone a precise, knowledge of its line parameters at all wavelengths is required.

The ozone parameters for this edition of HITRAN have been updated in two steps. First, the parameters of spectroscopic models, line positions, intensities, and lower-state energy levels resulting from analyses of experimental spectra have been introduced into the S\&MPO (Spectroscopy and Molecular Properties of Ozone) information system [119]. The S\&MPO offers various tools for spectral simulations and for comparisons with experimental records. At the initial stage of the work, line lists were generated using empirically-fitted model parameters of the effective Hamiltonians (EH) which considered nearby strongly interacting levels and of the corresponding effective dipole transition moments (ETM). This procedure was necessary to obtain complete sets of transitions as only a limited number of unblended lines could be accurately measured in congested ozone spectra. State-of-the-art studies of ozone laboratory spectra in the infrared have been recently reviewed by Barbe et al. [120] and Mikhailenko [121]. Note that most of the compilations of ozone data in the infrared accessible via the VAMDC (Virtual Atomic and Molecular Data Center) portal $[122,123]$ are also based on the S\&MPO line lists. This is the case of recent improvements in HITRAN and also in GEISA [49] for which the last release essentially contained the earlier 2015 update from the S\&MPO.

In the second step, the line lists were validated by simulating atmospheric spectra as discussed in Section 2.3.3.

The new or completely recalculated bands of the principal isotopologue of ozone implemented in HITRAN2016 are summarized in Table 5. The present release of the ozone data contains 29,006 new lines and 46,688 updated lines of ${ }^{16} \mathrm{O}_{3}$ (a graphical overview in the $200-5000 \mathrm{~cm}^{-1}$ region can be seen in Fig. 8).

\subsubsection{Cold bands}

2.3.1.1. 5- $\mu m$ region: tetrad $\{(002),(101),(200),(030)\}-(000)$. The 5$\mu \mathrm{m}$ range is of particular importance for the retrieval of atmospheric ozone from ground-based and satellite observations, as this is the second strongest absorbing range in the ozone spectrum after the $10-\mu \mathrm{m}$ bands. For this reason, the consistency of ozone in- tensities between the $5-\mu \mathrm{m}$ and $10-\mu \mathrm{m}$ regions as well as with UV ozone absorption is a major issue that has been a subject of many discussions ([124,125] and references therein). The line parameters in previous HITRAN versions for the strongest bands, i.e. the first triad $v_{1}+v_{3}, 2 v_{1}, 2 v_{3}$, in the 5 - $\mu \mathrm{m}$ interval were from the work by Flaud et al. [126]. Subsequently, the FTS spectra were re-visited by Barbe et al. [127] though the corresponding data had not been included in HITRAN. Recently new measurements and analyses have been performed by the GSMA (Groupe de Spectrométrie Moléculaire et Atmosphérique) team from Reims University [120,128]. In 2015, a major update of line positions and intensities based on the list generated together with the Laboratory of Theoretical Spectroscopy of the Institute of Atmospheric Optics (Tomsk) was introduced in the S\&MPO [119]. For the $2 v_{1}$ and $2 v_{3}$ bands, the difference in the integrated intensities, $S_{V}$, exceeded $6 \%$ between S\&MPO and HITRAN2012. The analysis [128] covering the $1856-2273 \mathrm{~cm}^{-1}$ region also included the $3 v_{2}$ band, which is coupled with $v_{1}+v_{3}$ via an accidental Coriolis interaction. Another resonance accounted for in this list explains the anomalously strong $\Delta K_{\mathrm{a}}=5$ lines that have been detected in atmospheric observations by Goldman et al. [129]. Janssen et al. [125] reported a comparative study of the sensitivity of FTIR ground-based measurements of the atmospheric ozone concentration with respect to line parameters included in the HITRAN2012, GEISA2015 and S\&MPO databases in 10- and 5$\mu \mathrm{m}$ regions. They have concluded that "only S\&MPO gives an entirely consistent result at the $\pm 1 \%$ level". Consequently, we include the S\&MPO line positions and intensities in the present HITRAN release. This should improve both intensity distribution in the ro-vibrational lines of the $5-\mu \mathrm{m}$ bands and their consistency with the $10-\mu \mathrm{m}$ range. The line-shape parameters for all the lines were estimated using the semi-empirical procedure described in the HITRAN2008 paper. This model largely relies on a slightly revised empirical model for the fundamental bands from Ref. [130].

2.3.1.2. $2335-2617 \mathrm{~cm}^{-1}$ : bands $\{(120),(021)\}-(000)$. Another spectral interval where S\&MPO and HITRAN2012 ozone data differ significantly is for the $2 v_{2}+v_{3}, v_{1}+2 v_{2}$ bands in the $2335-2617 \mathrm{~cm}^{-1}$ range. Previous HITRAN versions included the MIPAS list [131] for 
Table 5

HITRAN2016 ozone update summary: principal isotopologue ${ }^{16} \mathrm{O}_{3}$.

\begin{tabular}{|c|c|c|c|}
\hline Band $v^{\prime}-v^{\prime \prime}$ & $\mathrm{N}$ & Region $\left(\mathrm{cm}^{-1}\right)$ & $S_{\mathrm{v}}$ \\
\hline $001-010$ & 1781 & $281.14-377.51$ & $1.255 \times 10^{-21}$ \\
\hline $100-010$ & 619 & $295.42-446.63$ & $2.646 \times 10^{-23}$ \\
\hline 030-020 & 2558 & $598.26-804.86$ & $1.942 \times 10^{-21}$ \\
\hline $101-020$ & 6 & $656.12-725.68$ & $4.937 \times 10^{-25}$ \\
\hline $002-100$ & 1779 & $899.45-1176.42$ & $3.233 \times 10^{-21}$ \\
\hline $101-100$ & 3604 & $911.32-1215.49$ & $6.100 \times 10^{-20}$ \\
\hline $101-001$ & 3457 & $921.37-1211.67$ & $5.148 \times 10^{-21}$ \\
\hline $002-001$ & 4379 & 926.56-1193.79 & $1.651 \times 10^{-19}$ \\
\hline $200-100$ & 3966 & $940.22-1229.80$ & $3.141 \times 10^{-21}$ \\
\hline 030-100 & 20 & $956.13-1170.40$ & $1.916 \times 10^{-23}$ \\
\hline $200-001$ & 2964 & $956.68-1235.44$ & $6.852 \times 10^{-21}$ \\
\hline 030-001 & 15 & $957.33-1201.66$ & $2.254 \times 10^{-24}$ \\
\hline $002-010$ & 467 & $1302.52-1435.34$ & $3.750 \times 10^{-23}$ \\
\hline $101-010$ & 1637 & $1347.52-1438.73$ & $9.393 \times 10^{-22}$ \\
\hline 030-010 & 369 & $1366.12-1475.95$ & $2.268 \times 10^{-23}$ \\
\hline $200-010$ & 102 & $1366.62-1541.76$ & $5.305 \times 10^{-24}$ \\
\hline $002-000$ & 6486 & $1856.83-2280.54$ & $1.019 \times 10^{-19}$ \\
\hline $200-000$ & 6122 & $1909.98-2340.81$ & $3.020 \times 10^{-20}$ \\
\hline $101-000$ & 7067 & $1921.44-2328.94$ & $1.194 \times 10^{-18}$ \\
\hline 030-000 & 105 & $2022.87-2272.37$ & $4.092 \times 10^{-22}$ \\
\hline 021-000 & 1482 & $2335.99-2440.14$ & $4.360 \times 10^{-22}$ \\
\hline $120-000$ & 2095 & $2395.22-2616.83$ & $3.063 \times 10^{-22}$ \\
\hline 013-010 & 2459 & $2889.23-3090.28$ & $4.735 \times 10^{-21}$ \\
\hline $112-010$ & 2046 & $2910.00-3121.94$ & $3.816 \times 10^{-22}$ \\
\hline 023-010 & 1355 & $3564.72-3657.05$ & $3.676 \times 10^{-22}$ \\
\hline 013-000 & 2673 & $3584.62-3791.77$ & $5.713 \times 10^{-21}$ \\
\hline $122-010$ & 620 & $3584.75-3751.75$ & $3.530 \times 10^{-23}$ \\
\hline $112-000$ & 3056 & $3600.25-3831.27$ & $8.306 \times 10^{-22}$ \\
\hline $400-010$ & 4 & $3623.87-3653.87$ & $6.743 \times 10^{-25}$ \\
\hline $311-100$ & 729 & $3739.97-3826.22$ & $2.398 \times 10^{-23}$ \\
\hline 005-100 & 508 & $3742.91-3726.13$ & $1.660 \times 10^{-23}$ \\
\hline $104-100$ & 51 & $3752.69-3863.55$ & $8.192 \times 10^{-25}$ \\
\hline $005-001$ & 278 & $3807.31-3917.54$ & $9.849 \times 10^{-24}$ \\
\hline $311-001$ & 436 & $3810.30-3946.73$ & $9.846 \times 10^{-24}$ \\
\hline $104-001$ & 950 & $3820.17-3894.94$ & $2.218 \times 10^{-22}$ \\
\hline 005-010 & 848 & $4128.33-4228.24$ & $2.371 \times 10^{-23}$ \\
\hline $311-010$ & 1018 & $4129.14-4228.62$ & $3.444 \times 10^{-23}$ \\
\hline $104-010$ & 66 & $4146.09-4228.28$ & $6.310 \times 10^{-25}$ \\
\hline 023-000 & 1192 & $4268.37-4357.21$ & $1.928 \times 10^{-22}$ \\
\hline $122-000$ & 724 & $4284.15-4454.93$ & $4.359 \times 10^{-23}$ \\
\hline $400-000$ & 8 & $4325.56-4398.58$ & $5.896 \times 10^{-25}$ \\
\hline $213-100$ & 63 & $4384.72-4423.50$ & $1.417 \times 10^{-24}$ \\
\hline $321-100$ & 126 & $4429.78-4466.82$ & $5.750 \times 10^{-24}$ \\
\hline $114-001$ & 306 & $4452.15-4508.72$ & $1.313 \times 10^{-23}$ \\
\hline $213-010$ & 503 & $4757.08-4825.80$ & $2.346 \times 10^{-23}$ \\
\hline $104-000$ & 1093 & $4802.98-4978.61$ & $7.789 \times 10^{-23}$ \\
\hline $005-000$ & 1514 & $4806.33-4938.21$ & $5.300 \times 10^{-22}$ \\
\hline $311-000$ & 1053 & $4827.65-4928.49$ & $3.450 \times 10^{-22}$ \\
\hline
\end{tabular}

Note: $\mathrm{N}$ is the number of transitions, $S_{\mathrm{v}}$ is the integrated intensity in units of $\mathrm{cm}^{1} /\left(\right.$ molecule $\left.\cdot \mathrm{cm}^{2}\right)$.

$2 v_{2}+v_{3}$ and older data from Flaud et al. [126] for $v_{1}+2 v_{2}$. S\&MPO data in this range were initially based on the analyses of Barbe et al. [132]. Subsequently, new Reims FTS measurements with a better signal-to-noise ratio [120] suggested an empirical intensity scaling factor of 0.86 for these bands. The resulting line list is provided for this release of HITRAN. Recently, accurate $a b$ initio dipole moment surfaces of ozone were constructed by Tyuterev et al. [133] that allowed theoretical intensities to be obtained from variational nuclear-motion calculations which used the potential energy surface of Ref [134]. The shape of the $v_{1}+2 v_{2}$ band given by these calculations is qualitatively more consistent with the S\&MPO data incorporated in the new list of HITRAN2016 than HITRAN2012 (see Fig. 9).

2.3.1.3. 3584-3831 $\mathrm{cm}^{-1}$ range: $\{(013),(112)\}-(000)$ bands. The S\&MPO contains data for the $v_{2}+3 v_{3}$ and $v_{1}+v_{2}+2 v_{3}$ bands in the range $3584-3831 \mathrm{~cm}^{-1}$ based on a larger sample of assigned transitions [135] than the previous releases of HITRAN (see Table 4 of Babikov et al. [119]). The parameters can be found in the corresponding sections of the online S\&MPO site [119], and 5729 tran- sitions are now included that makes the integrated intensity of the $v_{1}+v_{2}+2 v_{3}$ band nearly 5 times stronger (Table 5) than in the previous release.

2.3.1.4. 4268-4399 $\mathrm{cm}^{-1}$ range: $\{(023),(122),(400)\}-(000)$ bands. In the range $4200-4525 \mathrm{~cm}^{-1}$, only the $3 v_{1}+v_{3}$ [136] and $2 v_{1}+2 v_{2}+v_{3}$ [137] cold bands were included in the previous releases of HITRAN, whereas the $2 v_{2}+3 v_{3}, v_{1}+2 v_{2}+2 v_{3}$ and $4 v_{1}$ bands in the 4268-4399 $\mathrm{cm}^{-1}$ range were missing, although they correspond to a total integrated intensity of $2.4 \times 10^{-22} \mathrm{~cm}^{-1} /$ molecule $\mathrm{cm}^{-2}$. The corresponding analyses $[121,138]$ are as yet unpublished, but the line parameters can be found at the online S\&MPO site [119] and are now incorporated in HITRAN2016.

2.3.1.5. 4802-4929 $\mathrm{cm}^{-1}$ range: $\{(005),(311),(104)\}-(000)$ bands. Line parameters for the $5 v_{3}, 3 v_{1}+v_{2}+v_{3}$ and $v_{1}+4 v_{3}$ bands around $4900 \mathrm{~cm}^{-1}$ are calculated for the HITRAN database using the spectroscopic parameters of Flaud et al. [139]. These bands were then re-analyzed using new spectra recorded in 


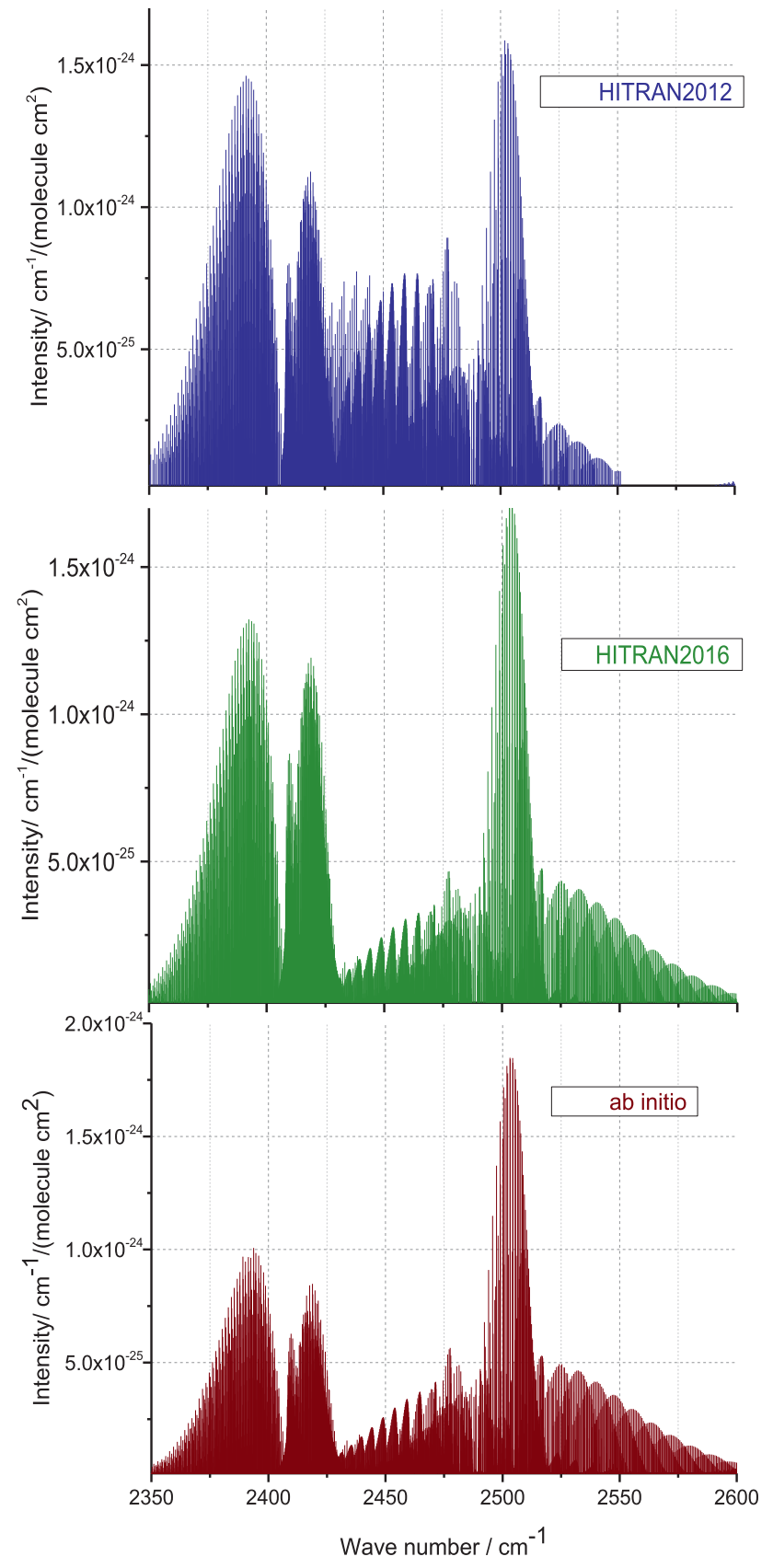

Fig. 9. Comparison of HITRAN2012 and HITRAN2016 ozone line lists in the range of $2 v_{2}+v_{3}$ and $v_{1}+2 v_{2}$ bands with recent ab initio calculations using the dipolemoment surface of Tyuterev et al. [133] that provides qualitative confirmation of the rotational distribution of intensities in the $v_{1}+2 v_{2}$ band given by HITRAN2016.

Reims [120] resulting in a new set of the Hamiltonian parameters for the strongly coupled $\{(005),(104),(311)\}$ vibration states. The corresponding line lists have been generated using these improved spectroscopic parameters. Unpublished Hamiltonian parameters can be found in the S\&MPO system at http://smpo.iao.ru/ 1408x675/en/lev/par/1/19/. The (S\&MPO-HITRAN2012) differences in the line positions are up to $0.065 \mathrm{~cm}^{-1}$. The intensity ratios for three cold bands are shown in Fig. 10.

\subsubsection{Hot bands}

2.3.2.1. $281-447 \mathrm{~cm}^{-1}$ (dyad $-v_{2}$ ). The ozone spectrum in the range 281-447 $\mathrm{cm}^{-1}$ is dominated by hot $v_{3}-v_{2}$ and $v_{1}-v_{2}$ bands whose intensities were underestimated in the previous HITRAN releases compared to the S\&MPO line list [120] which was generated using the empirical transition moments parameters of Birk et al. [140]. The S\&MPO parameters are confirmed by the recent $a b$ initio intensity calculations [133] and the corresponding update is thus included in the present HITRAN release.

2.3.2.2. 598-1541 $\mathrm{cm}^{-1}$ : hot bands towards the tetrad region. The unpublished version of the newly determined tetrad $\{(002),(101),(200),(030)\}$ parameters by Tyuterev et al. [128] can be found at the S\&MPO online site (http: //smpo.iao.ru/1396x659/en/lev/par/1/6/). Using this set, 14 hot bands in the range 598-1541 $\mathrm{cm}^{-1}$ that go from the (010), (020), (001) and (100) states to the tetrad were re-calculated and are now included in the present HITRAN release with a significantly augmented number of transitions. The transition moment parameters of Ref. [128] for $\{(101),(030)\}-(010)$ bands and those extrapolated from cold bands [141] were used.

2.3.2.3. 2889-3122 $\mathrm{cm}^{-1}$ range: $\{(013),(112)\}-(010)$ bands; $3564-$ $3752 \mathrm{~cm}^{-1}$ range: $\{(023),(122)\}-(010)$ bands. In the ranges 2889 $3122 \mathrm{~cm}^{-1}$ and $3564-3752 \mathrm{~cm}^{-1}$ the $\{(013),(112)\}-(010)$ and $\{(023),(122)\}-(010)$ hot bands are updated using the same upperstate EH parameters as for the corresponding cold bands and the ETM parameters of Mikhailenko et al. [142]

2.3.2.4. 3623-4229 $\mathrm{cm}^{-1}$ : hot bands to the $V=5$ triad $\{(005),(311),(104)\}$. New line lists for 14 hot bands in the range $3623-4229 \mathrm{~cm}^{-1}$ corresponding to the transitions from the $(010$, (001) and (100) states to the updated [120] triad \{(005),(311),(104)\} of five vibrational quanta are also included.

2.3.2.5. 4384-4826 $\mathrm{cm}^{-1}$ : missing hot bands to (213),(321) and (114) upper states. The coupled vibrational states $\{(213),(321),(114)\}$ have been re-analyzed in Ref [143] with the FTS spectra recorded in Reims. Using these upper-state parameters we now provide four associated hot bands [119] in the range $4384-4826 \mathrm{~cm}^{-1}$ that were missing in previous HITRAN versions. The ETM parameters were extrapolated from the corresponding cold bands: for $2 v_{2}+v_{2}+3 v_{3}-v_{1}$ and $v_{2}+v_{2}+4 v_{3}-v_{3}$ from Ref [144], for $2 v_{2}+v_{2}+3 v_{3}-v_{2}$ from Ref [145], and for $3 v_{2}+2 v_{2}+v_{3}-v_{1}$ from Ref [137].

\subsubsection{Validations and empirical corrections}

As mentioned in the introduction to Section 2.3, the second step was a validation of effective model calculations by a comparison of simulated absorption cross-sections with observed spectra. To this end, high-resolution laboratory FTS spectra of Reims and Kitt-Peak (up to $5700 \mathrm{~cm}^{-1}$ ) were used alongside MklV balloon atmospheric spectra [146], using similar procedures to those described by Toon et al. in [147]. The MkIV balloon spectra cover $650-5650 \mathrm{~cm}^{-1}$ simultaneously in every spectrum, so every window is fitted in every spectrum, making it easier to quantify window-to-window biases.

No major problems were seen with the initial $\mathrm{O}_{3}$ data set prepared for HITRAN2016, at least for strong and medium-strength bands. The new line list provides improved spectral fits in the $2100 \mathrm{~cm}^{-1}$ region as compared with HITRAN2012 and also better consistency with other bands in terms of the retrieved $\mathrm{O}_{3}$ amounts in the $2000-2200 \mathrm{~cm}^{-1}$ region. But it was found that empirical corrections were needed to some line positions to reduce residuals between observed and simulated cross-sections. These corrections concern a limited sample of about $0.1 \%$ transitions and are essentially of two types. First, small line position corrections are typically of the order of $\sim 10^{-3} \mathrm{~cm}^{-1}$ up to $10^{-2} \mathrm{~cm}^{-1}$ in a few cases. The most extreme deviations were corrected already for HITRAN2016, but additional corrections will be done in the near future. Note that these corrections are not reflected in Table 5 where 


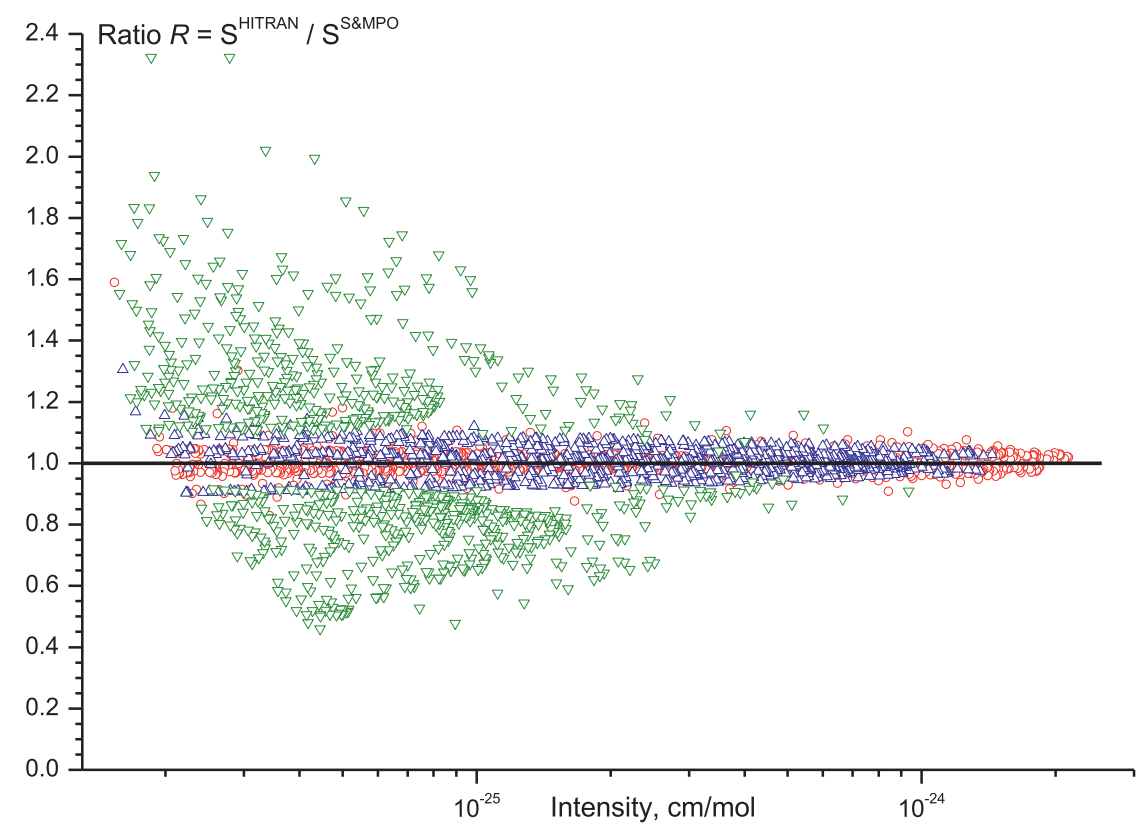

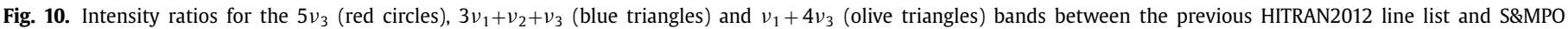
data that are incorporated in the new HITRAN2016 release (color can be seen in online version of paper).

only updates for entire bands rather than individual transitions were carried out. A total of 75,694 lines for ${ }^{16} \mathrm{O}_{3}$ have been added or updated (this includes corrections from Table 5 and the empirical corrections discussed in this subsection). Note that there are 289,340 lines of the principal isotopologue of ozone in total in the HITRAN2016 edition. Secondly, there is an indication that pressureinduced line shift parameters may need to be added or extended in the future.

It is known [120] that for the fundamental bands the effective model fits reproduce observed spectra within experimental accuracy $\left(\sim 10^{-4} \mathrm{~cm}^{-1}\right.$ for line positions), but the precision of the calculations gradually decreases for higher wavenumber ranges. The RMS of available line position fits increases on average to $\sim 0.001 \mathrm{~cm}^{-1}$ in the $3000 \mathrm{~cm}^{-1}$ region, to $\sim 0.002 \mathrm{~cm}^{-1}$ around $4000 \mathrm{~cm}^{-1}$ and to $\sim 0.003 \mathrm{~cm}^{-1}$ in the $5000 \mathrm{~cm}^{-1}$ range, primarily because of accidental resonances. A typical example is a sharp resonance of the (111) state interacting with the "dark" (040) state for $\left[J, K_{a}\right]=[44,4]$ that was not included in the effective model. This resonance produces a perturbation of $0.048 \mathrm{~cm}^{-1}$ in line positions and mixing of the line intensities between coupled transitions. The upper-state energy level and six corresponding P, Q, R- transitions have been empirically corrected both for the cold $v_{1}+v_{2}+v_{3}$ and hot $v_{1}+v_{2}+v_{3}-v_{2}$ bands. The major part of the relatively large line position corrections $\left(\sim 0.02-0.12 \mathrm{~cm}^{-1}\right.$ for about ten transitions) to previous HITRAN line lists occurs near $2.5-\mu \mathrm{m}$ and corresponds to the analysis of $\{(103),(004),(310)\}-(000)$ bands by Perrin et al. [148]. This region is extremely complicated to model because of the strong coupling of 9 bands including some "exotic" resonances, for instance $\{(103),(004)\}$ with (310) and $\{(211),(202),(032)\}$. Analyses of new spectra recorded in Reims for these bands are in progress as outlined in Refs. [120,121], but are not yet finalized. In the meantime, empirical corrections have been applied to corresponding series of levels, mostly around $4000 \mathrm{~cm}^{-1}$, and to associated transitions. This process removes some noticeable residuals between simulated and observed absorption cross-sections.

Relative intensities of various bands play a key role in terms of the consistency of retrieved $\mathrm{O}_{3}$ amount from different spectral windows. From this point of view, the HITRAN2016 line list is certainly much better than its predecessors. For example, the
HITRAN2016/S\&MPO linelist produces 8\% larger retrieved amounts around $2182 \mathrm{~cm}^{-1}$ than previous line lists, bringing this region into better consistency with others. This improvement can be attributed to the major update of the tetrad $\{(101),(200),(002),(030)\}$ bands $[120,128]$ in the $5-\mu \mathrm{m}$ region and confirms the conclusion of Janssen et al. [125] concerning the internal consistency of the S\&MPO list that is now incorporated in HITRAN2016. The $3000 \mathrm{~cm}^{-1}$ region yields the lowest retrieved $\mathrm{O}_{3}$ amounts, about $5 \%$ lower than average, in fits to MklV limb spectra and Kitt Peak lab spectra [146]. This indicates possible errors in line widths or intensities, since the errant line positions were corrected.

\subsubsection{Future work}

There remain several issues for future work. The problem of absolute ozone intensities is still an important challenge [120,124] requiring very precise measurements and ab initio calculations with absolute uncertainties better than $1-3 \%$. This concerns particularly the isotopic species. New data on ${ }^{17} \mathrm{O}$ - and ${ }^{18} \mathrm{O}$ - containing isotopologues will be produced in the near future. Modeling of the complicated $2.5 \mu \mathrm{m}$ region $[120,121,148]$ must be significantly improved. In general, for the spectral intervals beyond the fundamental bands, the effective models do not provide experimental accuracy for all lines. In order to obtain line position accuracy that is better than $0.001 \mathrm{~cm}^{-1}$ in the FTS range, which is important for atmospheric applications, we could extend experimental determination of ro-vibrational levels and systematically replace transition frequencies by available empirical $\left(E-E^{\prime}\right) / h$ values as described in Refs. $[149,150]$ where this approach was used for the analysis and line lists of CRDS spectra. Further extension of ozone spectral data to highly-excited states near the dissociation threshold (Ref. [151] and references therein) would also be important for the understanding of the dynamics of ozone formation and depletion [152] and for interpretation of satellite observations involving nonLTE effects in the upper atmosphere.

\section{4. $\mathrm{N}_{2} \mathrm{O}$ (molecule 4)}

The $\mathrm{N}_{2} \mathrm{O}$ line list has been updated using the recently-published line list for the ${ }^{14} \mathrm{~N}_{2}{ }^{18} \mathrm{O}\left({ }^{14} \mathrm{~N}^{14} \mathrm{~N}^{18} \mathrm{O}\right)$ isotopologue [153]. In the immediate future, we will also add (for the first time) the line list 
that has already been constructed for the ${ }^{15} \mathrm{~N}_{2}{ }^{16} \mathrm{O}\left({ }^{15} \mathrm{~N}^{15} \mathrm{~N}{ }^{16} \mathrm{O}\right)$ isotopologue based on Ref. [154]. Both line lists were generated using the results of the global modeling of the line positions and intensities within the framework of the method of effective operators. The polyad model of effective Hamiltonian (EH) [155] was used in both cases. The parameters of the effective Hamiltonians were fit to the line positions collected from the literature. The global RMS of the line position fit for ${ }^{15} \mathrm{~N}_{2}{ }^{16} \mathrm{O}$ is $0.0011 \mathrm{~cm}^{-1}$ and for ${ }^{14} \mathrm{~N}_{2}{ }^{18} \mathrm{O}$ is $0.0016 \mathrm{~cm}^{-1}$. The uncertainties in the fitted line positions depend on the quality of experimental data and vary from $0.0046 \mathrm{~cm}^{-1}$ to $2 \times 10^{-6} \mathrm{~cm}^{-1}$.

The line intensities were calculated using fitted sets of the effective dipole moment parameters for a particular isotopologue where possible. Note that the line intensity measurements for rare isotopologues of $\mathrm{N}_{2} \mathrm{O}$ are very scarce. Therefore, based on the theoretical results of Ref. [156] (in which contributions due to isotopic substitution to the values of the effective dipole moment parameters were shown to be negligible) the majority of the line intensities were calculated using the effective dipole moment parameters of the principal isotopologue [157]. The partition sums were taken from Ref. [112] for ${ }^{14} \mathrm{~N}_{2}{ }^{18} \mathrm{O}$ and calculated using direct summation for ${ }^{15} \mathrm{~N}_{2}{ }^{16} \mathrm{O}$ [154].

It is well known that the nitrous oxide EH polyad model does not work in a totally satisfactory manner because of the existence of a number of Coriolis and anharmonic interpolyad resonance interactions (see, for example, Refs. [158,159]). But usually these kinds of interactions take place in the high-wavenumber region for the weak bands. Adopting an intensity cutoff of $10^{-29} \mathrm{~cm}^{-1} /$ molecule $\mathrm{cm}^{-2}$ at $296 \mathrm{~K}$, including the isotopic abundance factors $1.986 \times 10^{-3}$ for ${ }^{14} \mathrm{~N}_{2}{ }^{18} \mathrm{O}$ and $1.32 \times 10^{-5}$ for ${ }^{15} \mathrm{~N}_{2}{ }^{16} \mathrm{O}$, overcomes the problem of the interpolyad resonance perturbations in practice because the most affected transitions are omitted. Nevertheless, line parameters for the very weak bands have to be used with caution.

For the ${ }^{14} \mathrm{~N}_{2}{ }^{18} \mathrm{O}$ isotopologue, Toth's [160] lines given in HITRAN2012 are retained and only new line parameters from the line list of Ref. [153] are added to HITRAN2016. Line positions from the line lists of Refs. [153,154] are assigned uncertainty code $6\left(0.000001-0.00001 \mathrm{~cm}^{-1}\right)$ in the MW region and $3(0.001-$ $\left.0.01 \mathrm{~cm}^{-1}\right)$ in the IR region. For the line intensities from these line lists, the uncertainty code 3 (>20\%) is adopted.

Line-shape parameters as well as Rosenkranz [35] line-mixing coefficients for the main isotopologue lines belonging to the $v_{3}$ ro-vibrational band are taken from the work of Loos et al. [161]. These parameters originate from a multispectrum fit of air broadened Fourier-Transform transmittance measurements at ambient temperature. The experimental data of Loos et al. was fitted using a quadratic speed-dependent Voigt model based on the HartmannTran profile $[7,8,14]$, extended to account for line mixing in the Rosenkranz first-order perturbation approximation [35]. As a result, air-broadening, air-speed-dependence, air pressure shift and line-mixing parameters were retrieved in the spectral range 2184.8 to $2251.6 \mathrm{~cm}^{-1}$ for P- and R-branch lines up to $J^{\prime \prime}=40$ and 39 , respectively.

\subsection{CO (molecule 5)}

In order to improve the quality and consistency (between bands and isotopologues) of the intensities of the lines of carbon monoxide in HITRAN, a new piece-wise dipole moment function (DMF) was created [162] using the direct fit approach that was developed in Ref. [163], supplemented with new ab initio calculations [162]. In order to ensure the best results, new experiments were carried out in Ref. [162] for the 4-0 band and the first measurements of the $6-0$ band were performed to add into the direct fit.
The line intensity and position calculations were performed using this new DMF in conjunction with the wavefunctions calculated from the experimentally-determined potential energy function (PEF) from Coxon and Hajigeorgiou [164]. Calculations were carried out using version 8.0 of LEVEL [165].

The new dipole moment function has proven to be a substantial improvement over the one used previously. In this edition of the database it was used to calculate HITRAN intensity values in all the bands of all isotopologues. Fig. 6 of Li et al. [162] shows a comparison of the available experimental data in different bands of the principal isotopologue, with new calculations, HITRAN2012, and ab initio calculations. It is clear that intensities from Li et al. agree much better with experimental values than previous data. This also becomes important for the consistency of intensities between the isotopologues (due to the use of the same dipole moment function for all of them); previously a lot of lines for the principal isotopologues had intensities from accurate laboratory measurements whereas minor isotopologue intensities were still calculated with outdated dipole moment functions. Finally, the new work allows one to add a few more bands and also line lists for radioactive isotopologues of carbon monoxide, which, as we mentioned in the $\mathrm{CO}_{2}$ section, are provided in static files rather than through the HITRANonline interface.

Apart from the intensities, the Li et al. line list features: a) line positions calculated based on the potential energy function from Ref. [164]; b) air- and self-broadening parameters from a sophisticated semi-empirical approach, c) air-induced shift obtained for all the lines by extrapolating measurements carried out in the 2-0 band by Devi et al. [166], where the Hartmann and Boulet [167] approach was used for extrapolating; d) widths (and their temperature dependencies) and shift due to pressure of $\mathrm{H}_{2}$ and $\mathrm{CO}_{2}$ important for planetary and combustion research were also provided employing semi-empirical approaches similar to the ones used for air and self pressure-induced values.

We have adapted the Li et al. line list for this edition of the HITRAN database with a few notable changes:

1) Since the original $\mathrm{Li}$ et al. line list was aimed at hightemperature applications, it was truncated here with an intensity cutoff of $10^{-31} \mathrm{~cm}^{-1} /\left(\right.$ molecule $\cdot \mathrm{cm}^{-2}$ ) (in natural abundance of the isotopologues) for all the lines except those in the MW region where a cutoff of $10^{-42} \mathrm{~cm}^{-1} /\left(\right.$ molecule $\left.\cdot \mathrm{cm}^{-2}\right)$ was used to satisfy some astrophysical applications.

2) It was found that there was a minor compatibility issue between LEVEL and PEF from Coxon and Hajigeorgiou [164]. This resulted in a growing (with rotational quantum number) deviation of the calculated line positions from their real values. This has now been fixed.

3) The line positions from the high-accuracy experiments that employ best calibration standards, including frequency combs, were used wherever available. In the first overtone, data from Pollock et al. [168] were used, in the second overtone data from Refs. $[169,170]$ were employed, while in the fifth overtone Tan et al. line positions were taken when available [171].

4) When high-quality experimental measurements of the air- and self-broadening and shift parameters were available in $\mathrm{HI}$ TRAN2012, they were taken in place of the semi-empirical values from Li et al. This includes the values for the speeddependent Voigt (with line mixing) profile for the first overtone from Devi et al. [166,172].

5) Helium-broadening parameters, their temperature dependence and pressure shift due to He were added. The broadening parameters were taken from Sinclair et al. [173] who provide both experimental values and values obtained from a fit to a semiempirical model. We used experimental values where available and semi-empirical values for all other transitions. Although 
Table 6

Scheme of HITRAN2016 update of ${ }^{12} \mathrm{CH}_{4}$ and ${ }^{13} \mathrm{CH}_{4}$. All regions not given in the table remain identical to the HITRAN2012 edition.

\begin{tabular}{|c|c|c|c|}
\hline Range $\left(\mathrm{cm}^{-1}\right)$ & Positions & Intensities & Line shapes \\
\hline $0-1370$ & $\begin{array}{l}\text { HIT12, with many hot bands removed. P1-P0 } \\
\left(v_{4} \text { band only), P1-P1, P2-P2, P3-P3, P2-P1 }\right. \\
\text { and P3-P2 from MeCaSDa [186], }{ }^{13} \mathrm{CH}_{4} v_{2} \\
\text { band restored from HIT08 }\end{array}$ & $\begin{array}{l}\text { P1-P0 ( } v_{4} \text { band only), P1-P1, P2-P2, P3-P3 } \\
\text { and P3-P2 from MeCaSDa [186], P2-P1 from } \\
\text { HIT08 }\end{array}$ & $\begin{array}{l}\text { HIT12 when available or } \\
\text { algorithm from Ref. [176] }\end{array}$ \\
\hline $1370-4000$ & $\begin{array}{l}\text { HIT12 with many line positions fixed using } \\
\text { HIT08,S Tyuterev et al. [187] and empirical } \\
\text { adjustments of HIT12 to properly fit KP } \\
\text { spectra. Several missing lines restored from } \\
\text { HIT08. }\end{array}$ & HIT12 & HIT12 \\
\hline $4000-4315$ & $\begin{array}{l}\text { HIT08 with many lines improved using some of } \\
\text { the preliminary results from Birk et al. [188], } \\
\text { and occasionally HIT12 }\end{array}$ & $\begin{array}{l}\text { HIT08 with many lines improved using some of } \\
\text { the preliminary results from DLR Birk et al. } \\
\text { [188] }\end{array}$ & HIT08 and in some cases HIT12 \\
\hline $4499-4630$ & HIT12 mixed with Devi et al. [189] & HIT12 mixed with Devi et al. [189] & \\
\hline $4670-5300$ & $\begin{array}{l}\text { Nikitin et al. [190] with } \\
10^{-27} \mathrm{~cm}^{-1} /\left(\text { molecule } \cdot \mathrm{cm}^{-2}\right) \text { cutoff applied in } \\
4800-5000 \mathrm{~cm}^{-1} \text { region }\end{array}$ & & Lyulin et al. [191] \\
\hline $5300-5550$ & Nikitin et al. [192] & & Lyulin et al. [191] \\
\hline $5550-5855$ & $\begin{array}{l}\text { GOSAT list [193] for }{ }^{12} \mathrm{CH}_{4} \text {. Other isotopologues } \\
\text { HIT12 }\end{array}$ & & $\begin{array}{l}\text { GOSAT list [193], or algorithm } \\
\text { from Ref. [176] }\end{array}$ \\
\hline $5855-6250$ & $\begin{array}{l}\text { GOSAT list [193] with some lines from Nikitin } \\
\text { et al. [194] and HIT12 based on validation for } \\
{ }^{12} \mathrm{CH}_{4} \text {. For }{ }^{13} \mathrm{CH}_{4} \text { Starikova et al. [195]. }\end{array}$ & & $\begin{array}{l}\text { GOSAT list [193], Lyulin et al. } \\
\text { [191] or algorithm from Ref. } \\
\text { [176] }\end{array}$ \\
\hline $7920-8250$ & CRDS measurements Béguier et al. [196] & & Algorithm from Ref. [176] \\
\hline $9028-10,435$ & FTS measurements Béguier et al. [197] & & Algorithm from Ref. [176] \\
\hline
\end{tabular}

Note: HIT16 = new HITRAN edition, HIT08 = HITRAN2008 edition [198], HIT12 = HITRAN2012 edition [1], KP= Kitt Peak FTS lab spectra.

these measurements were carried out only in the fundamental band, they were used here for all lines ignoring vibrational dependence. The temperature dependence of this broadening was taken to be 0.6 for all the lines based on the average value of the measurements by Picard-Bersellini et al. [174]. Finally, the pressure induced shifts are based on measurements of Luo et al. [175] in the fundamental band and extrapolated wherever possible to other bands using the Hartmann and Boulet [167] approach.

\section{6. $\mathrm{CH}_{4}$ (molecule 6)}

The state-of-the art of methane data in the previous HITRAN releases has been described by Brown et al. [176]. Since then, several new studies have been in progress or have been published both for cold and room-temperature spectra [177-183] and hot spectra measurements $[184,185]$, although many experimental spectra have not yet been fully analyzed. Toon et al. [147] have reported some deficiencies in available databases including HITRAN2012 [1] by simulations of solar occultation spectra, acquired by the JPL MkIV Fourier transform spectrometer from balloon, covering the $650-5650 \mathrm{~cm}^{-1}$ region. These deficiencies have been addressed in this new edition, which includes superior spectral parameters and new lines and bands for ${ }^{12} \mathrm{CH}_{4}$ and ${ }^{13} \mathrm{CH}_{4}$. At this time no update was made to $\mathrm{CH}_{3} \mathrm{D}$. An overall brief picture of the update is given in Table 6, while the details are given in the subsequent sections. Note that only regions where revision to HITRAN2012 was carried out are shown. In the regions not mentioned in the table, HITRAN2012 line parameters have been retained.

\subsubsection{Hot bands in the spectral range below $1370 \mathrm{~cm}^{-1}$}

The HITRAN2012 database exhibited some noticeable problems, including those with hot band lines of ${ }^{12} \mathrm{CH}_{4}$ in the low wavenumber range. The line list in this spectral region relies on calculations resulting from the effective Hamiltonian and dipole moment parameter fits, since extensive experimental line assignments have not been performed for many years. However, extrapolation errors have been introduced in some small spectral regions that were insufficiently well characterized. Recently, a new global fit of methane lines including high-temperature emission data in the
$1100-1500 \mathrm{~cm}^{-1}$ region was published [185]. This study included a huge number of new assignments and allowed a much more reliable modeling of hot-band lines. It has resulted in a determination of effective Hamiltonian parameters. The results were first used to update the MeCaSDa database [186] of calculated methane lines. Fig. 11 compares the resulting computed cross-sections with the HITRAN2012 and ExoMol [199] databases. This plot, in logarithmic scale, clearly displays that there was likely a problem in HITRAN2012 but better agreement of the new calculated line list with Exomol.

It is well known that measurement and modeling of line intensities in high-temperature spectra are quite difficult, particularly in non-Boltzmann conditions. In order to avoid large uncertainties in extrapolated intensities, as was the case for some hot bands in the previous HITRAN editions, we have checked new lists against $a b$ initio calculations. The line lists for hot bands resulting from new experimental spectra analyses by Amyay et al. [185]. were compared to first-principle variational calculations of line intensities by Rey et al. [200,201], based on the ab initio dipole moment and potential energy surfaces of Nikitin et al. [202-204] as reported in the TheoReTS database [205]. An example of comparison is given in Fig. 12. Those bands that were in good intensity agreement were considered sufficiently reliable to be incorporated in this new HITRAN release. Some other bands require further investigation and were not included. Note that, at this stage, these qualitative comparisons did not involve line assignment or line position validations.

This new calculated line list was thus used to replace some of the HITRAN2012 lines in order to generate the HITRAN2016 update, for the P1-P0 (Dyad-GS, for the $v_{4}$ band only), P1-P1 (DyadDyad), P2-P2 (Pentad-Pentad), P3-P3 (Octad-Pentad) and P4-P3 (Tetradecad-Octad) transitions (a schematic of the polyads can be seen in Fig. 2 of Ref. [5]). Table 7 shows the spectral regions and the number of lines that exist in MeCaSDa and what proportion of them was adapted for HITRAN2016. It is important to mention that validations against theoretical [205] and laboratory [185] spectra suggested adapting the P2-P1 line list from MeCaSDa, with intensities adapted from HITRAN2008 wherever possible.

It should also be noted that, recently, new highly-accurate measurements of pure rotation lines were performed in the Dunkerque 


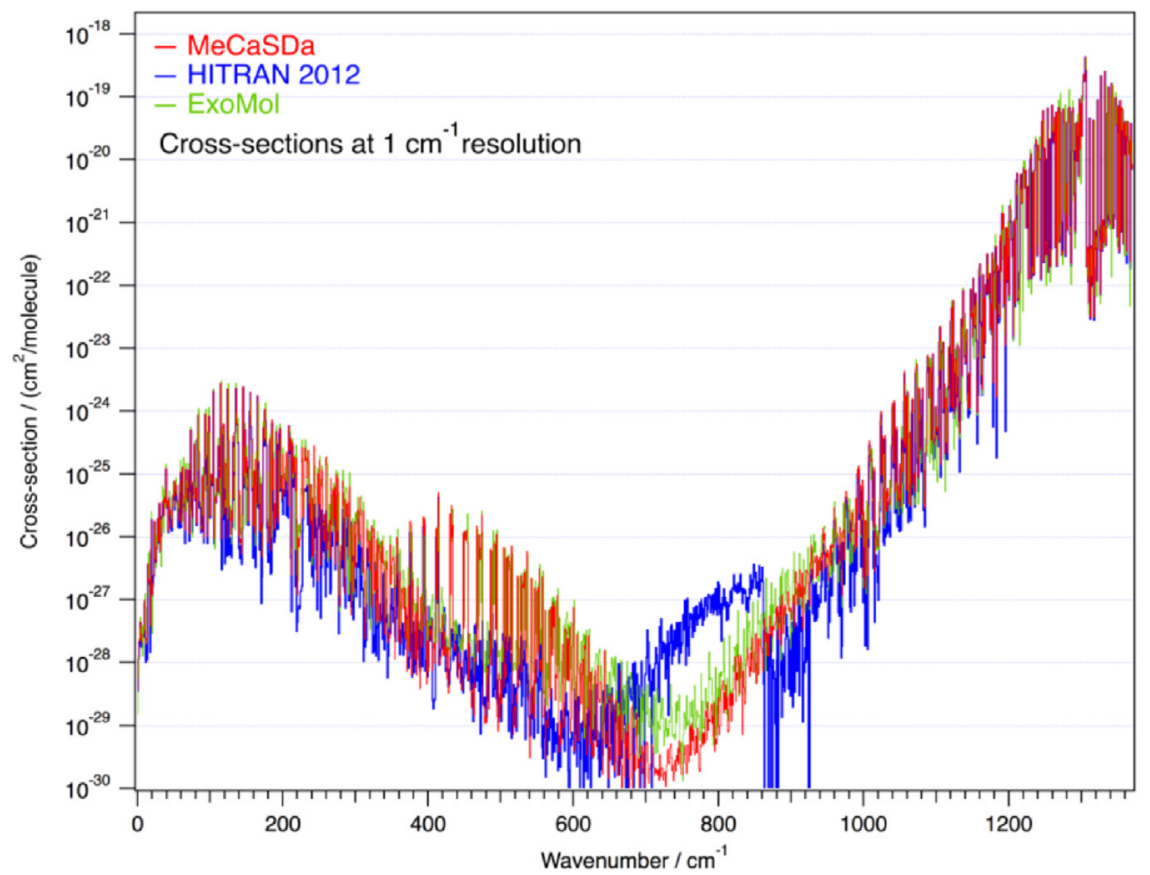

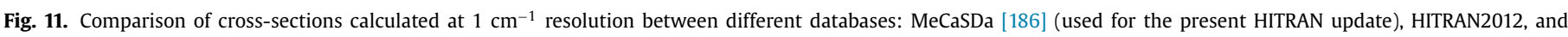
ExoMol [199].
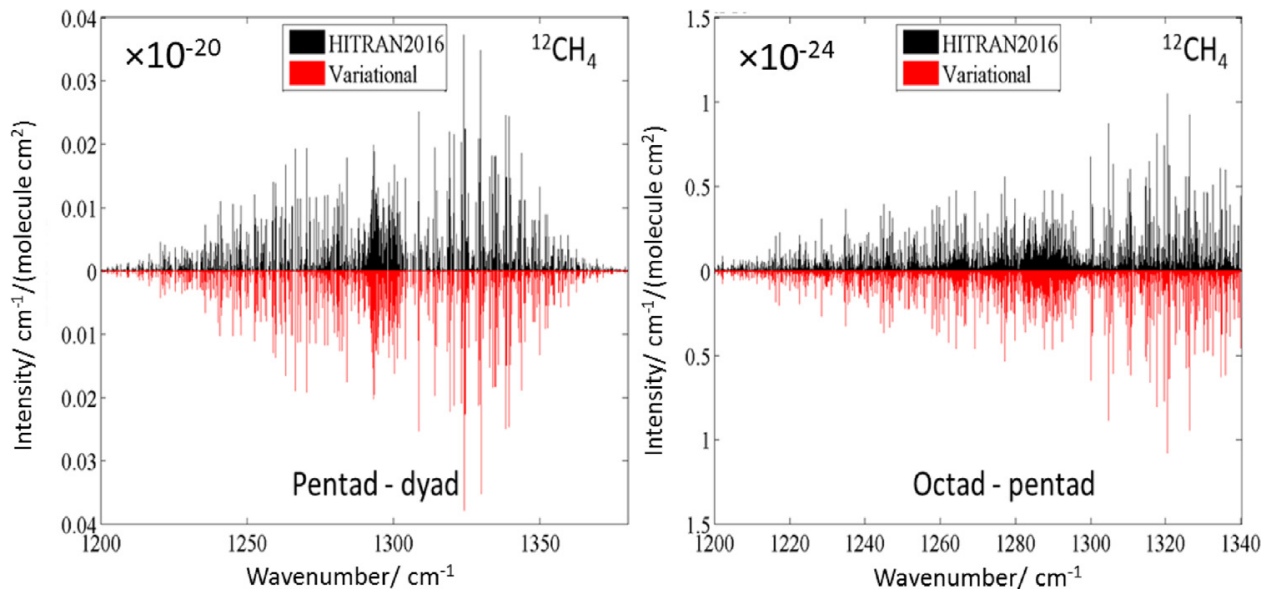

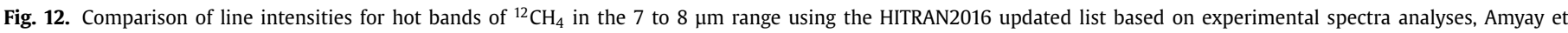

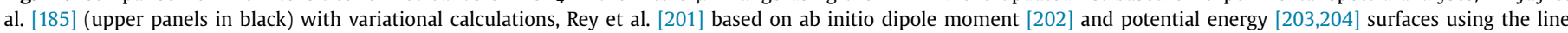
list included in the TheoReTS database [205] (lower panels upside down in red).

Table 7

Number of calculated ${ }^{12} \mathrm{CH}_{4}$ lines taken from the MeCaSDa database [186] for some transitions, up to a maximum $J$ value. The difference for the number of lines in the two databases is due to a different intensity cut-off with $I_{\min }$ down to $10^{-40} \mathrm{~cm} / \mathrm{molecule}$ in MeCaSDa. The weakest transitions were not retained for the present HITRAN update that includes $I_{\min }$ $=10^{-25}$ for P3-P2 and $10^{-30} \mathrm{~cm} /$ molecule for other hot bands.

\begin{tabular}{lllll}
\hline Transition & $\mathrm{P} 1-\mathrm{P0}\left(v_{4}\right)$ & $\mathrm{P} 1-\mathrm{P} 1$ & $\mathrm{P} 2-\mathrm{P} 2<300 \mathrm{~cm}^{-1}$ & $\mathrm{P} 3-\mathrm{P} 2<1370 \mathrm{~cm}^{-1}$ \\
\hline$J_{\max }$ & 30 & 30 & 30 & 30 \\
\# lines (MeCaSDa) & 13,315 & 54,677 & 457,620 & $2,175,406$ \\
\# lines(HIT16) & 9662 & 9721 & 10,701 & 950 \\
\hline
\end{tabular}

group [206]. These lines have been included in the global fit leading to the effective Hamiltonian parameters used for the present update.

\subsection{2. $1370-4000 \mathrm{~cm}^{-1}$ region}

This region mainly includes the $v_{2}$ band, the Pentad bands and relatively weak bands at the lower part of the Octad. As discussed by Toon et al. [147], HITRAN2012 data were plagued with erroneous line positions for lines of intermediate strength, many of which had been substantially superior in the HITRAN2008 edition of the database. It was found that a large number of these lines had been assigned line position uncertainty code 1 (i.e. between 0.1 and $1 \mathrm{~cm}^{-1}$ ) and originate from the model used in Daumont et al. [207]. For HITRAN2016, wherever possible in the Pentad re- 
gion, these transitions were reverted to HITRAN2008 and, where this was not possible, line positions from Tyuterev et al. [187] were used. Unfortunately not all problematic line positions were easy to identify on a global scale by just the uncertainty index. Validations against Kitt Peak FT spectra recorded at multiple thermodynamic conditions have shown that more errors associated with line positions remained although the offending lines were not labeled with a low uncertainty code. The most obvious outliers have been identified and fixed either by employing Ref. [187] or adjusting line positions to fit the low-pressure KP experimental spectra. This provided a significant improvement of the residuals, including those discussed in Toon et al. [147].

\subsection{3. $4000-4630 \mathrm{~cm}^{-1}$ (correspond to strongest octad bands)}

Just as for the Pentad region, HITRAN2012 data had substantial errors in line positions for lines of intermediate strength, while better values actually existed in the HITRAN2008 edition of the database. However, unlike the Pentad region, the intensities of lines in the Octad region were also often inferior to those from HITRAN2008. Also, unlike the Pentad region, reverting line positions of HITRAN2012 lines with uncertainty code 1 to the HITRAN2008 values did not always work. One should also keep in mind that HITRAN2008 was not better than HITRAN2012 everywhere in this region. In addition to lacking ${ }^{13} \mathrm{CH}_{4}$ lines in that region, HITRAN2008 contained many duplicated transitions. Often a transition would appear as an empirical unassigned line as well as predictions from Hilico et al. [208]. With that being said, some observable transitions were also missing in HITRAN2008.

The following procedure was therefore developed:

1) In the $4000-4315 \mathrm{~cm}^{-1}$ region, the HITRAN2008 line list was used as the starting point. Many unassigned empirical lines were then given assignments and lower-state energies from corresponding predicted Hilico et al. [208] lines, while predicted lines (usually with inaccurate line position and intensity) were removed. In the region $4190-4315 \mathrm{~cm}^{-1}$, parameters of lines with the worst remaining residuals (with respect to low-pressure Kitt Peak FTS spectra) were then replaced with the preliminary results of Birk et al. [188], which used quantum assignments of yet unpublished theoretical work [209] that was not always the same as in HITRAN2012.

2) In the $4315-4499 \mathrm{~cm}^{-1}$ region, HITRAN2012 parameters were retained.

3) In the 4499-4630 $\mathrm{cm}^{-1}$ region, a mixture of HITRAN2012 with parameters from Devi et al. (where available) was used [189].

Fig. 13 shows fits to TCCON spectra measured from Park Falls, WI, in March 2005 at a solar zenith angle of 58 deg., in windows used by TCCON to retrieve CO. The left panels show fits to the region occupied by the CO P-branch. The right panels show fits in the R-branch region. The CO lines are less than 10\% deep and so are mainly hidden by stronger $\mathrm{CH}_{4}$ and solar lines. The upper panels show fits using HITRAN2012 $\mathrm{CH}_{4}$; the lower panels with the HITRAN2016 $\mathrm{CH}_{4}$ linelist. The rms spectral fits and the peak residuals are significantly reduced in both windows with the HITRAN2016 linelist. The retrieved $\mathrm{CH}_{4}$ amounts (written over the top right of each panel) are much more consistent between the two windows using the HITRAN2016 linelist(0.1\%), than when using HITRAN2012 (2\%). It implies that the $2016 \mathrm{CH}_{4}$ intensities are much more selfconsistent across these two regions than in HITRAN2012. With that being said, there is still large room for improvements. A more consistent Octad list in terms of line parameters and assignments is planned to be worked out for the next HITRAN update.

\subsubsection{Tetradecad range $4800-6250 \mathrm{~cm}^{-1}$}

Contrary to the low-energy range, in the Tetradecad range and above, positions and intensities are mostly experimental values.
The Tetradecad of methane contains 14 bands with 60 strongly coupled sub-bands with very congested spectra difficult to analyze because of numerous resonance perturbations. The previous HITRAN2012 line list was mainly based on the analysis of Refs. $[210,211]$, which included about 3000 assigned lines. This was only a partial assignment with relatively large uncertainties for about $70 \%$ of weak sub-bands. In HITRAN2016, major updates have been included in four spectral intervals. Long-path Fourier Transform spectra $(L=1600 \mathrm{~m})$ in the lower Tetradecad interval 4800 $5300 \mathrm{~cm}^{-1}$ recorded in Reims have been re-analyzed by Nikitin et al. [190]. The list includes transitions with line positions adjusted according to their measured values and transitions with calculated line positions, all intensities being derived from the model fit. For HITRAN2016 in the $4800-5000 \mathrm{~cm}^{-1}$ region, transitions with intensities greater than $10^{-27} \mathrm{~cm}^{-1} /\left(\right.$ molecule $\left.\cdot \mathrm{cm}^{-2}\right)$ were retained.

In the next interval, $5300-5550 \mathrm{~cm}^{-1}$, the list contains mostly observed positions and intensities. The analysis is in progress [192], but a preliminary empirical line list is included in the present release because the previous versions were quite poor in this range.

In the interval 5550-6250 $\mathrm{cm}^{-1}$, a recent version of the GOSAT line list [193] is included. In the $2 v_{3}$ region (5855$6250 \mathrm{~cm}^{-1}$ ), the GOSAT line list [193] was supplemented with predominantly experimental line parameters of the WKLMC list (Wang-Kassi-Leshchishina-Mondelain-Campargue) obtained from high-sensitivity laser measurements in Grenoble [212]. The previous GOSAT assignments [211] included in the HITRAN2012 list have been considerably extended in the recent work [194] based on new analyses in the $5855-6250 \mathrm{~cm}^{-1}$ range. The modeling of these complicated spectra was accomplished using an approach combining $a b$ initio calculations with effective Hamiltonian (EH) fits. Initial values of the EH parameters were first computed from the molecular potential energy surface of Nikitin et al. [203] using irreducible tensor techniques $[213,214]$ via the high-order contact transformation method as described by Tyuterev et al. [187]. This information has been only partially adapted for HITRAN2016. Indeed, the validations against experimental and atmospheric spectra (similar to those done in Toon et al. [147]) showed that the GOSAT line list [193] produces better residuals than the one from Ref. [194], although the latter one is substantially more complete in terms of both amount of lines and proportion of assigned lines. In the end, the GOSAT line list [193] was supplemented with some lines from Ref. [194] and occasionally with HITRAN2012. For instance, very accurate line positions measured with frequency comb in Zolot et al. [215] were retained. In the future, a more sophisticated combination of the GOSAT line list [193] and the one from Ref. [194] will be performed. For broadening, a combination of parameters from Lyulin et al. [191], GOSAT line list and HITRAN2012 was used.

\subsubsection{Higher wavenumber ranges}

In the Icosad range $\left(6300-7500 \mathrm{~cm}^{-1}\right)$, the WKLMC list provided empirical values of the lower-state energy levels. Recently, on the basis of global variational $a b$ initio spectra predictions [180], about 13,000 transitions of 108 new bands have been assigned [216] in the WKLMC list at $80 \mathrm{~K}$ and $296 \mathrm{~K}$. The assigned experimental list at $80 \mathrm{~K}$ as well as calculated ones are provided as Supplementary Material of Rey et al. [216]. These assignments will be transferred and extended in the WKLMC list at $296 \mathrm{~K}$ and included in the next update of HITRAN.

Fig. 14 gives an overview comparison between the HITRAN2012 and HITRAN2016 lists of ${ }^{12} \mathrm{CH}_{4}$ above $7920 \mathrm{~cm}^{-1}$. New roomtemperature measurements by Cavity Ring Down Spectroscopy (CRDS) in the $7920-8250 \mathrm{~cm}^{-1}$ interval have allowed better characterizing of the $1.25-\mu \mathrm{m}$ methane transparency window [21]. In the Triacontad region (8250-9028 $\mathrm{cm}^{-1}$ ), line parameters relying on FTS measurements by Brown [42] remain unaltered with re- 

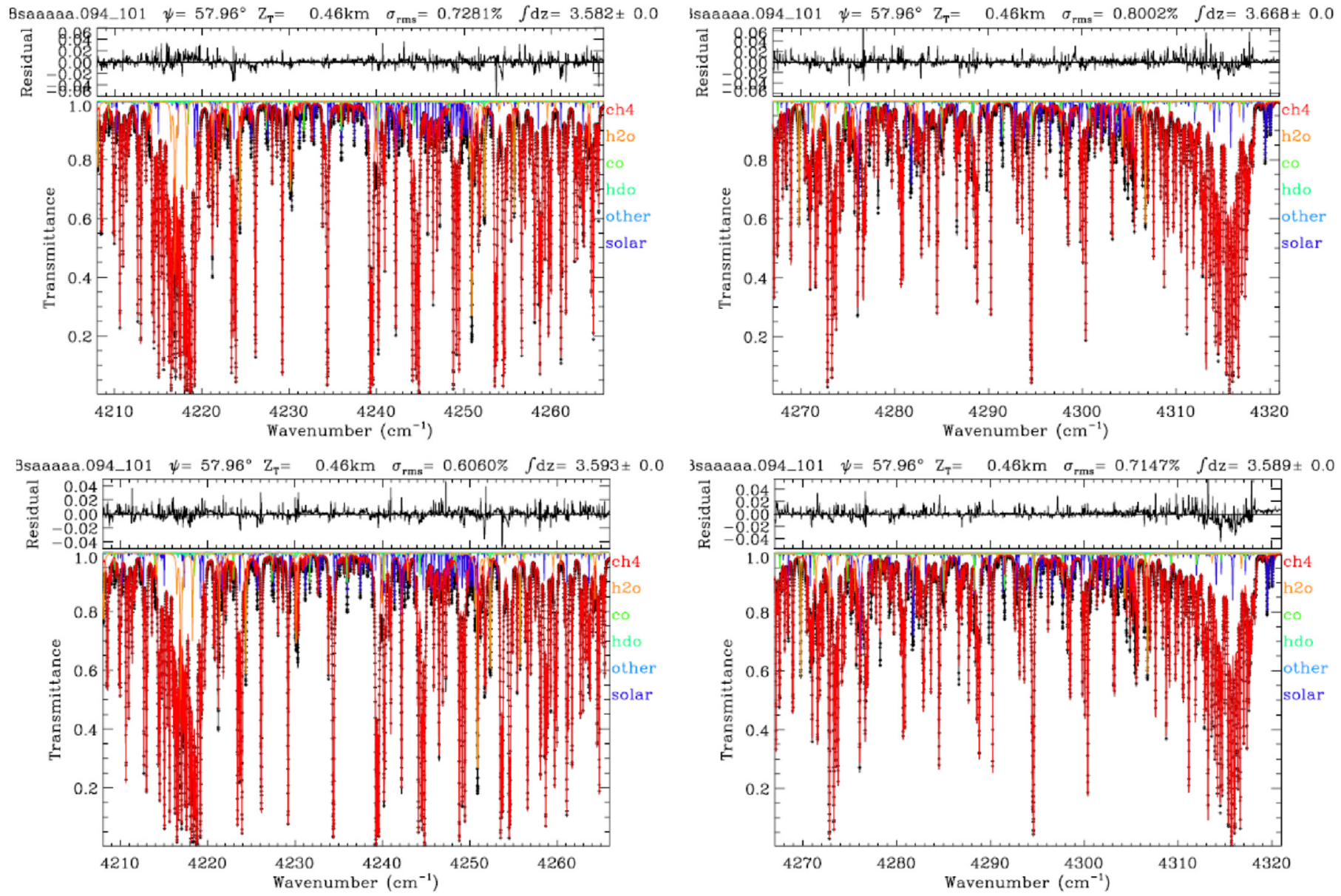

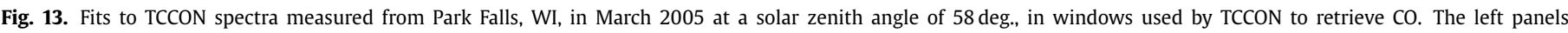

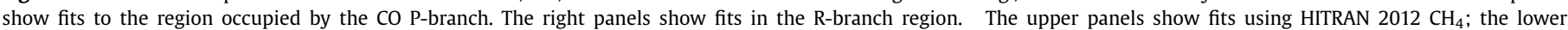
panels with the HITRAN2016 $\mathrm{CH}_{4}$ linelist. Note different scales.

spect to HITRAN2012. Above $9028 \mathrm{~cm}^{-1}$, new FTS line parameters are adopted in the region of the $3 v_{3}$ band $\left(9028-9200 \mathrm{~cm}^{-1}\right)$ [22]. From the same FTS spectra, the first empirical line list was retrieved in the Tetracontad region $\left(9520-10,435 \mathrm{~cm}^{-1}\right)$ [22]. It is worth mentioning that between $7920 \mathrm{~cm}^{-1}$ and $10,923 \mathrm{~cm}^{-1}$ no assignments or empirical lower-state energy values are provided except for a few tens of transitions in the region of the $3 v_{3}$ band near $9046 \mathrm{~cm}^{-1}$. Complementary information in this case can be provided by variational ab initio predictions [31]. The estimated error for line positions in the best first-principle calculation in the $1-\mu \mathrm{m}$ range is on average $\sim 0.3-2 \mathrm{~cm}^{-1}$; that is not sufficient for high-resolution applications. However, intensities are in qualitatively good agreement with observations, see Fig. 15. Recent ab initio intensity results [217] for the strongest ${ }^{12} \mathrm{CH}_{4}$ lines below $7600 \mathrm{~cm}^{-1}$ have confirmed accurately measured intensity values to $1-3 \%$ on average for the "stable" transitions non perturbed by accidental resonances. Predicted spectra [31] include lower-state energies for all lines and can be recalculated for various temperature conditions that make it suitable for low- and mediumresolution modeling of observations in planetary atmospheres similar to those reported for Titan in Ref [45].

Finally, in the $10,923-11,502 \mathrm{~cm}^{-1}$ spectral range, the HITRAN lists reproduce line parameters derived by Intracavity Laser Absorption Spectroscopy (ICLAS) [220]. For the ICLAS data [176,220], empirical lower-state energy values were derived by the twotemperature-method [221].

\subsection{6. ${ }^{13} \mathrm{CH}_{4}$ isotopologue}

In HITRAN2012, the entire $v_{2}$ band of ${ }^{13} \mathrm{CH}_{4}$ (centered at about $1550 \mathrm{~cm}^{-1}$ ) was accidentally dropped from the compilation. This band is now restored back from HITRAN2008 [198].

For the ${ }^{13} \mathrm{CH}_{4}$ isotopologue, new measurements and analyses in the $3750-4700 \mathrm{~cm}^{-1}$ octad range have been reported by Brown et al. [222] for temperatures between 80 and $296 \mathrm{~K}$. This included over 4700 positions and 3300 intensities for assigned observed transitions. The line list based on the analysis and spectra fits of this range contains 9500 calculated lines. It is available as supplementary material attached to Ref. [222] and will be incorporated in the next HITRAN update.

First assignments of the strongest bands at the highwavenumber boundary of the ${ }^{13} \mathrm{CH}_{4}$ Tetradecad $\left(5852-6200 \mathrm{~cm}^{-1}\right.$ region dominated by $2 v_{3}$ ) have been reported by Starikova et al. [195]. Differential Absorption spectra (DAS) recorded in Grenoble [223] at $80 \mathrm{~K}$ and $296 \mathrm{~K}$ have been used for this analysis. For the $80 \mathrm{~K}$ spectra, about 2900 lines of 3717 observed lines were assigned. The theoretical model in both spectral intervals, 3750$4700 \mathrm{~cm}^{-1}$ and $5852-6200 \mathrm{~cm}^{-1}$, was based on the combined approach [187] using ab initio predictions for line positions and intensities [224] with subsequent EH fits, which is similar to that for the main ${ }^{12} \mathrm{CH}_{4}$ isotopologue as outlined in Section 2.6.4 Supplementary materials of Ref. [195] contain a partially-assigned experimental DAS list at $80 \mathrm{~K}$. For the present HITRAN2016 release, the assignments were extended for the $296 \mathrm{~K}$ list. Note that many observed absorption features in the congested spectrum could have 


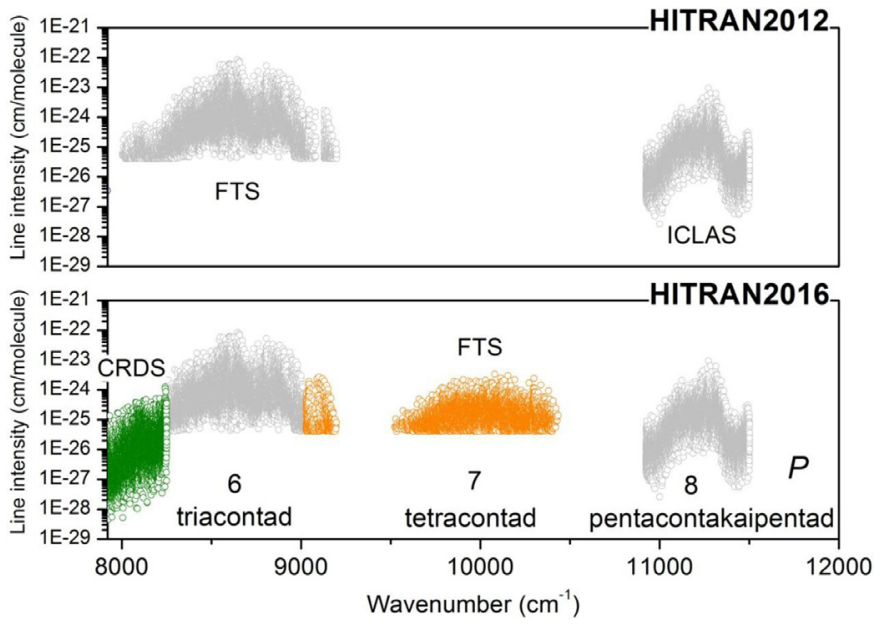

Fig. 14. Overview comparison of the HITRAN line list of ${ }^{12} \mathrm{CH}_{4}$ above $7920 \mathrm{~cm}^{-1}$ in the 2012 and 2016 editions. In this region, line parameters all have an empirical origin: CRDS between 7920 and $8250 \mathrm{~cm}^{-1}$ [196], FTS by Brown in the 8250$9028 \mathrm{~cm}^{-1}$ interval [218] by Béguier et al. in the $9028-10,923 \mathrm{~cm}^{-1}$ interval [197], and ICLAS between 11,000 and $11,500 \mathrm{~cm}^{-1}$ [219]. The different polyad and corresponding quantum numbers, $\mathrm{P}$, are indicated. (The polyad number $\mathrm{P}$ is equal to $2\left(V_{1}+V_{3}\right)+V_{2}+V_{4}$, where $V_{i}$ are the normal mode vibrational quantum numbers).

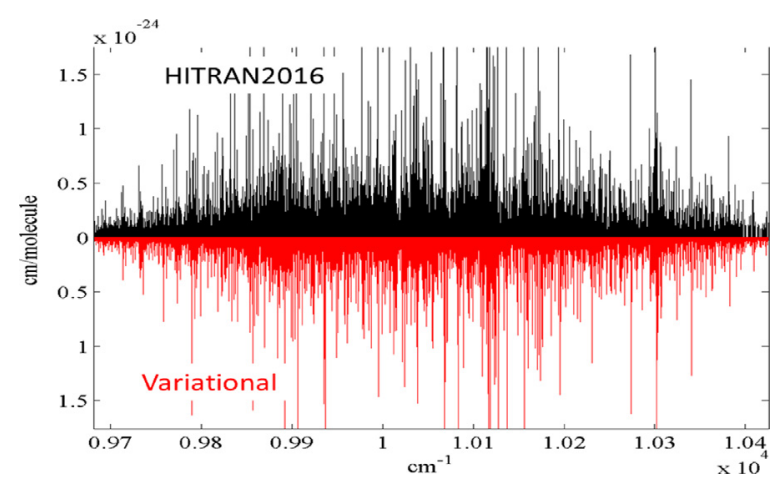

Fig. 15. Comparison of unassigned experimental line list of ${ }^{12} \mathrm{CH}_{4}$ in the Tetracontad range (Béguier et al. [197]) included in HITRAN2016 (upper panels in black) with variational calculations, Rey et al. [205] based on ab initio dipole moment [202], and potential energy [203,204] surfaces using the line list included in the TheoReTS database [205] (lower panel upside down in red).

contributions from various overlapping transitions. As the assignment criteria, the ratio $R=I_{\text {re-calc }} /\left\{I_{o b s}(296 \mathrm{~K})\right\}$ was used as well as the coincidence of $J_{\text {low }}$ obtained theoretically and via the empirical 2T-method. Here $I_{\text {re-calc }}$ stands for intensities re-calculated from the $80 \mathrm{~K}$ DAS list via the Boltzmann factor. In doubtful cases, but with consistent $J_{\text {low }}$ values, we have kept empirical line parameters. For big $R$ deviations and inconsistent $J_{\text {low }}$ values, a default value $E_{\text {low }}$ $=333.3333 \mathrm{~cm}^{-1}$ was applied. The self- and air- line broadening were calculated following the method described in the GOSAT paper [211].

Fig. 16, giving the overview of ${ }^{13} \mathrm{CH}_{4}$ lines in the $2 v_{3}$ range, shows that most of the strong and medium lines in the HITRAN2016 release have consistent assignments.

An extension of this work using the approach of Ref. [216] is in progress for the Icosad range of ${ }^{13} \mathrm{CH}_{4}$ [225].

\subsection{7. $\mathrm{CH}_{3} \mathrm{D}$ and other isotopologues}

The parameters for this singly-deuterated methane molecule have not been updated for the 2016 edition. We note a recent paper by Bray et al. [226] which indicated the need for updating the intensities of $\mathrm{CH}_{3} \mathrm{D}$ in the pure rotational band. We are considering this work for a future update of HITRAN.

\subsubsection{Line-shape parameters for methane}

Our knowledge of pressure broadening of methane by air, $\mathrm{N}_{2}$, $\mathrm{H}_{2}$, He, $\mathrm{CH}_{4}$, and other gases remains incomplete, particularly for the near-infrared and for weaker transitions in all spectral regions. Basic coefficients of Lorentz broadening (widths, shifts, temperature dependences) all vary as a function of the transition quantum numbers, and values obtained for the fundamental bands are not easily applicable to the very complicated polyads having multiple underlying vibrational states. Available measured values for Lorentz pressure broadened line-shape parameters from the previous edition of HITRAN were transferred into the new compilation, but most of the lines have been given crudely-estimated air- and self-broadened half widths, as described by Brown et al. [176]. Good theoretical models, confirmed by measurements, must be implemented in order to have accurate values, not the estimates used here for $99 \%$ of the present database. Since the release of the 2012 database, additional methane line-shape measurements have become available in several spectral regions for ${ }^{12} \mathrm{CH}_{4}$ [179,181,183,189,227-232], and $\mathrm{CH}_{3} \mathrm{D}$ [226,233,234], and these will be added in the future updates to HITRAN2016.

The recent study of Mendonca et al. [235] illustrates the growing evidence that for remote-sensing applications requiring the highest accuracies of line parameters to model the observed methane spectrum, Voigt lineshapes are inadequate. It is well known that collisional line mixing can significantly affect absorption spectral shapes, and line mixing cannot be neglected for accurate retrievals of atmospheric $\mathrm{CH}_{4}$ abundance. The HITRAN2012 database [1] had included the line-mixing parameters for $\mathrm{CO}_{2}, \mathrm{CO}$ and $\mathrm{O}_{2}$, however without providing guidance or tools for users to implement these parameters in their calculations. The new structure of the HITRAN database [236] and the HITRAN Application Programming Interface (HAPI) [5] provide the potential for the inclusion of line-mixing parameters in the database and provide tools for their implementation.

A theoretical approach proposed to model line-mixing effects for $\mathrm{CH}_{4}$ by Tran et al. [[237-239] has been successfully applied to calculate absorption in the $v_{3}, v_{4}$ and $2 v_{3}$ bands. The same model has been used to simulate the spectra in the $v_{2}+v_{3}$ band as a test to demonstrate whether it can be applied to other vibrational bands. Comparisons with spectra measured under different pressure conditions [189] show that line mixing only weakly affects the absorption spectral line shape of this band under these pressure conditions. Therefore, more calculations for other vibrational bands under large pressure conditions are needed to demonstrate if this model can be applied to various vibrational bands. While the work of Tran et al. ([239] and references therein) represents significant progress in understanding non-Voigt line shapes in methane, additional laboratory studies of line mixing, speed dependence and narrowing are required to provide the basic parameters for future methane compilations in the spectral regions most utilized for remote sensing.

\section{7. $\mathrm{O}_{2}$ (molecule 7)}

An extensive update of the HITRAN oxygen line list for the new edition makes use of the following significant progress on: (1) a self-consistent set of energy levels and line positions for the entire HITRAN oxygen line list; (2) lineshape, line mixing, collision induced absorption (CIA), and new speed-dependent Voigt (SDV) formalism for the A-band; (3) HT profile parameters for the B- bands. We also added the $b^{1} \Sigma_{g}^{+}(v=3)-X^{3} \Sigma_{g}^{-}(v=0)$ and $b^{1} \Sigma_{g}^{+}(v=2)$ $X^{3} \Sigma_{g}^{-}(v=1)$ bands.

\subsubsection{Line positions and energy levels}

A self-consistent set of spectroscopic constants from an updated isotopically invariant Dunham fit by Yu et al. in 2014 [240] were 


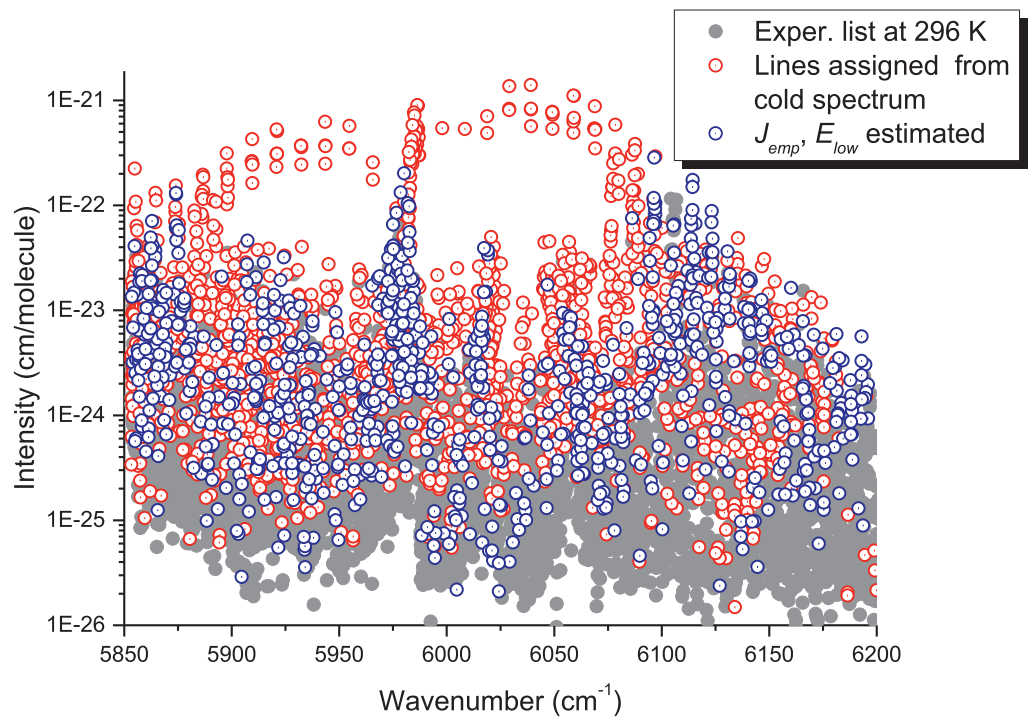

Fig. 16. Overview of assigned lines [195] in the experimental ${ }^{13} \mathrm{CH}_{4}$ DAS list [223] included in HITRAN2016 in the 1.66- $\mu \mathrm{m}$ range.

used to update line positions and energy levels throughout the entire HITRAN2012 oxygen line list, except for the so-called zerofrequency lines (due to Zeeman splitting of the ground state), see HITRAN1986 paper for details [241]. This updated Dunham fit was obtained by adding new microwave and infrared measurements [242,243] to the first global analysis of $\mathrm{O}_{2}$ by Yu et al. in 2012 [244] that simultaneously fits all available experimental line positions for all the bands and isotopologues from the microwave to UV region. The new microwave measurements [242] include 324 rotational transitions in the $a^{1} \Delta_{g} v=0$ and 1 states of the six $\mathrm{O}_{2}$ isotopologues and helped determine two more hyperfine parameters, the electric quadrupole interaction $e Q q$ and the nuclear spin-rotation interaction, $C_{I}$. The new infrared work [243] reported $1644 b^{1} \Sigma_{g}^{+}-X^{3} \Sigma_{g}^{-}$transition frequencies for the six $\mathrm{O}_{2}$ isotopologues and revealed a $0.2 \mathrm{~cm}^{-1}$ calibration error in the ${ }^{17} \mathrm{O}^{18} \mathrm{O}$ $v^{\prime}-v^{\prime \prime}=1-0$ Raman data of Edwards et al. [245]. It resolved discrepancies in the Raman data for ${ }^{16} \mathrm{O}^{17} \mathrm{O},{ }^{17} \mathrm{O}^{17} \mathrm{O}$, and ${ }^{17} \mathrm{O}^{18} \mathrm{O}$, and improved the vibrational parameterization of the ground electronic state.

A quantum number assignment error has been fixed in $\mathrm{HI}-$ TRAN2016 for 17 lines in the $\Delta N \Delta J=P O$ branch of the ${ }^{16} \mathrm{O}^{16} \mathrm{O}$ $a-X\left(v^{\prime}, v^{\prime \prime}\right)=(0,0)$ band. These lines were incorrectly labeled with a shift of two in $N^{\prime \prime}$ and $J^{\prime \prime}$ in HITRAN2012, as was pointed out in Yu et al. [240].

Band-by-band comparisons of the updated line positions and lower state energies versus the HITRAN2012 compilation were performed. For all the bands in the $a^{1} \Delta_{g}-X^{3} \Sigma_{g}^{-}$system (for which the data in HITRAN originate from Refs. [246-249], systematic differences, smaller than experimental uncertainties, were found for measured lines and expected deviations were found for extrapolated lines. As depicted in the upper left panel of Fig. 17, systematic differences of $0.0007 \mathrm{~cm}^{-1}$ were found for the ${ }^{16} \mathrm{O}^{16} \mathrm{O} a-X\left(v^{\prime}, v^{\prime \prime}\right)=(0,0)$ band. Similar deviations were found for all other bands except the A-band $\left(b-X\left(v^{\prime}, v^{\prime \prime}\right)=(0,0)\right)$ of ${ }^{16} \mathrm{O}^{16} \mathrm{O},{ }^{16} \mathrm{O}^{17} \mathrm{O}$ and ${ }^{16} \mathrm{O}^{18} \mathrm{O}$, where the data originated from Refs. [250,251] (see discussion below).

As shown in upper right, lower left and lower right panels of Fig. 17, deviations are very small for one sub-group of the A-band transitions while they are large for another sub-group. The subgroup of transitions with small deviations belongs to the ${ }^{P} \mathrm{O},{ }^{P} \mathrm{P},{ }^{P} \mathrm{Q}$ branches while the other sub-group with large deviations belongs to the ${ }^{N} O,{ }^{R} R,{ }^{R} Q{ }^{R} S$ and ${ }^{T} S$ branches.

\subsubsection{A-band region near $762 \mathrm{~nm}$}

The line list for the A-band in HITRAN2012 originates from the data from the Long et al. [250,251] papers. Here an update of the principal isotopologue magnetic dipole allowed transitions is given that incorporates a self-consistent model of collisional lineshapes, collisional line mixing and collision induced absorption in a multispectrum fit to a wide range of experimental data sets described in Drouin et al. [252] (hereafter referred to as Drouin 2017). The lineshape chosen was the speed-dependent Voigt profile and effects of line mixing were introduced as a fixed W-matrix derived from the theoretical line mixing formalism of Tran, Boulet and Hartmann in 2006 [253]. Strong correlation was observed between the scaled line-mixing parameterization and the collisioninduced absorption derived as remaining non-resonant absorption. Correlation was also observed between the line-by-line parameters correlations in the R-branch band-head region and the scaled line-mixing parameterization. The self and air-broadening parameters as well as air-pressure shift parameters do not deviate significantly from HITRAN 2012, with smooth trends that closely match the polynomial expressions of Long et al. that were used in the prior compilation. Unlike HITRAN 2012, minor erratic deviations of these parameters (which were minimized in the chosen scaling of the line mixing) are retained in the compilation for selfconsistency. The self-pressure shift parameters are provided for the first time in this HITRAN edition, and these values differ systematically by $0.006 \mathrm{~cm}^{-1} \mathrm{~atm}^{-1}$ from those given by Long et al. [251]. The air broadening temperature dependence is also systematically shifted to higher values in comparison to HITRAN 2012. The selfbroadening temperature dependence is also provided for this band for the first time in HITRAN and it is even more systematically shifted in comparison to published values from Brown \& Plymate [254]. Temperature dependences of the pressure-shift are also included in the new line-by-line parameterization; there is no prior parameterization for comparisons of these values. The final fitted line-by-line parameter is the 'speed-dependence' of the pressure broadening, which replaces the Dicke parameter determined in Long's work and included in the supplemental listing of HITRAN 2012. Qualitative comparison of these two 'narrowing' parameters shows that there is a similar $m$ dependence in each parameter set, each with a more pronounced slope at $m<0$ (the P-branch). Use of the speed-dependent Voigt allows this lineshape profile to be cast within the more encompassing Hartmann-Tran profile. Line-by-line line-mixing parameters (Rosenkranz parameters) were not fitted in 

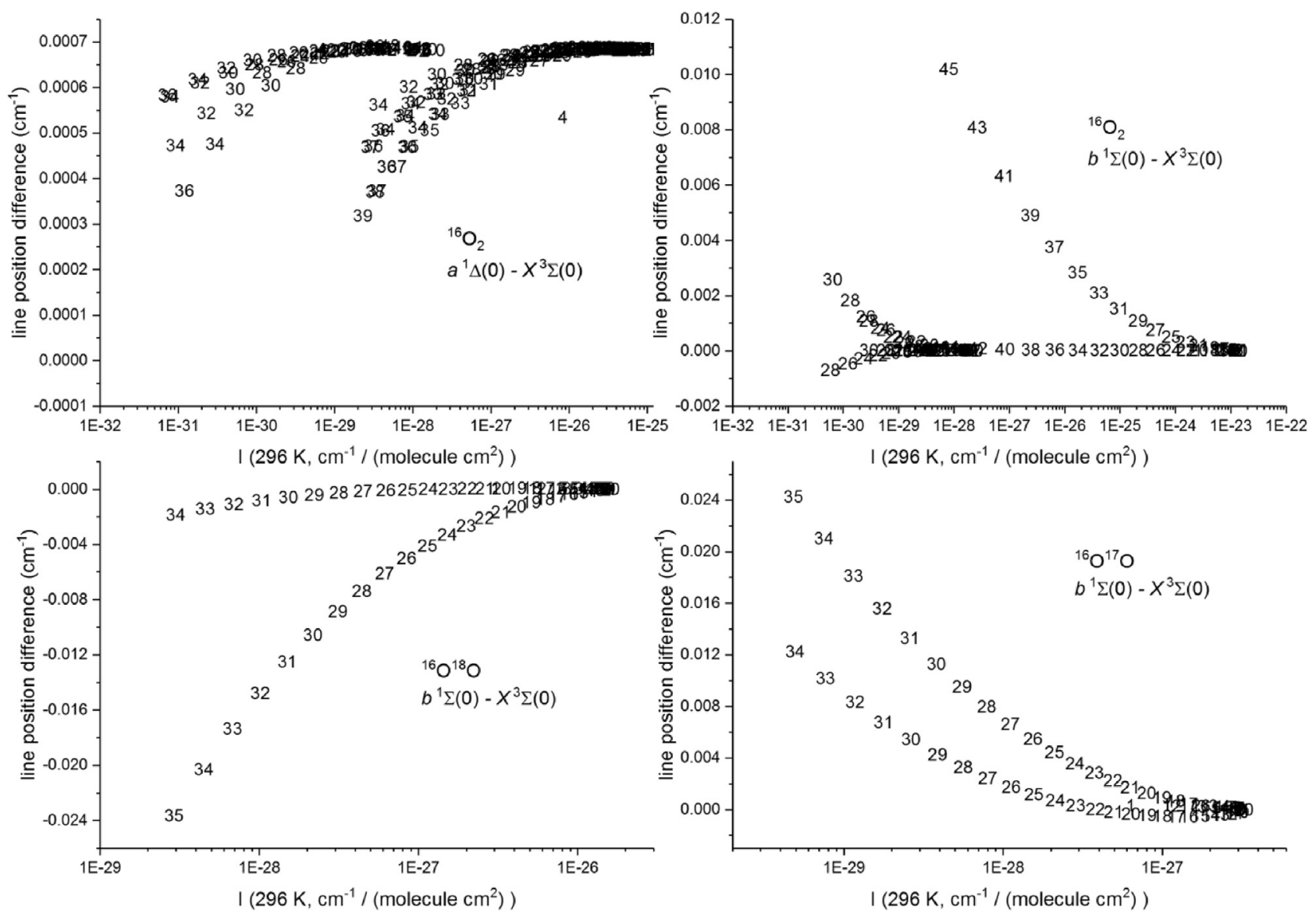

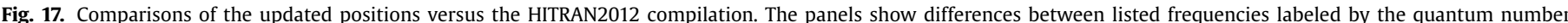

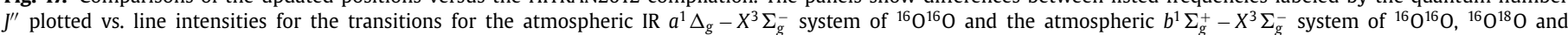

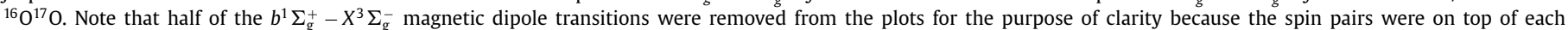
other.

Drouin 2017 [252], but rather a full W-matrix formalism based on theoretical work, empirically modified to improve the multispectrum fit quality, was implemented. The empirical corrections that attenuated line mixing at high rotational excitation (see Tran and Hartmann, [255]) were abandoned in favor of the removal of mixing between differing spin-states. The effects of this correction are clearly evident in the derived Rosenkranz parameters, which oscillate between even and odd $J$ (or $m$ ) values when the spin-changing collisional mixing is allowed, but follow a smooth trend with $J$ (or $m$ ) when they are removed. Predoi-Cross et al. [256,257] provide fitted room-temperature Rosenkranz parameters (and other lineshape parameters) that agree with the parameters of Drouin 2017. The modified line mixing parameterization significantly alters the derived collision-induced absorption, with this absorption appearing narrower and stronger, with a shape and extent that conforms more closely to the resonant band. The collision-induced absorption derived for air is provided separately in the corresponding section of the HITRAN database, see Section 4 for details.

For the convenience of HITRAN users, two transformations have been applied to the line-shape/line- mixing parameterization given by Drouin 2017. First, given the primary usage of the oxygen database to model the Earth's atmosphere, the foreign-broadening line-shape parameters presented in Drouin 2017 have been converted to air-broadening parameters using the binary mixing ratio $\left[\mathrm{N}_{2}\right]:\left[\mathrm{O}_{2}\right]=0.79: 0.21$. Second, the scaled, full-W-matrices for foreign and self line mixing and the associated temperature dependence were evaluated for $1 \mathrm{~atm}$ of air at four 'standard' temperatures and then composed into first order line-by-line Rosenkranz parameters. This representation of the line mixing is based on similar representations provided for the fine-structure line mixing in the microwave spectrum and is both easier to tabulate as well as more efficient to compute. We note that the multidimensional oxygen A-band cross-section table (ABSCO 5.0), now in use for the reduction of OCO-2 [64] atmospheric data, utilizes the parameterization of Drouin 2017 in its native form, and was calculated with the same source code as the multi-spectrum fitting program. The differences between the ABSCO 5.0 table and the HITRAN database are due to the two cosmetic modifications indicated above, as well as the replacement of line centers with the more precise values derived from the work of Yu et al. [240] described above.

\subsubsection{New addition of $b^{1} \sum_{g}^{+}(v=3)-X^{3} \sum_{g}^{-}(v=0)$ and $b^{1} \sum_{g}^{+}$} $(v=2)-X^{3} \sum_{g}^{-}(v=1)$ bands and merging in the UV lines for ${ }^{16} \mathrm{O}_{2}$

Atmospheric spectra can show where important bands are missing. Fig. 18 shows a high-air mass ground-based Kitt Peak spectrum fitted using the HITRAN 2012 line list. It is clear that the $b^{1} \Sigma_{9}^{+}(\mathrm{v}=3)-X^{3} \Sigma_{g}^{-}(\mathrm{v}=0)$ band of $\mathrm{O}_{2}$ at $0.58 \mu \mathrm{m}$ is missing in the compilation. The line list for this band was calculated and while there were data to calculate line positions [258], no information was available for intensities, and this atmospheric spectrum was used to scale the intensities to fit the observations. We also note that line positions used in HITRAN2016 for this band differ from those in Yu et al. [240]. This is due to the fact that the measurements in Ref. [258] were carried out using a relatively high pressure of oxygen. It is accounted for in the fit here but not completely in Ref. [240]. Broadening and shift parameters for this 

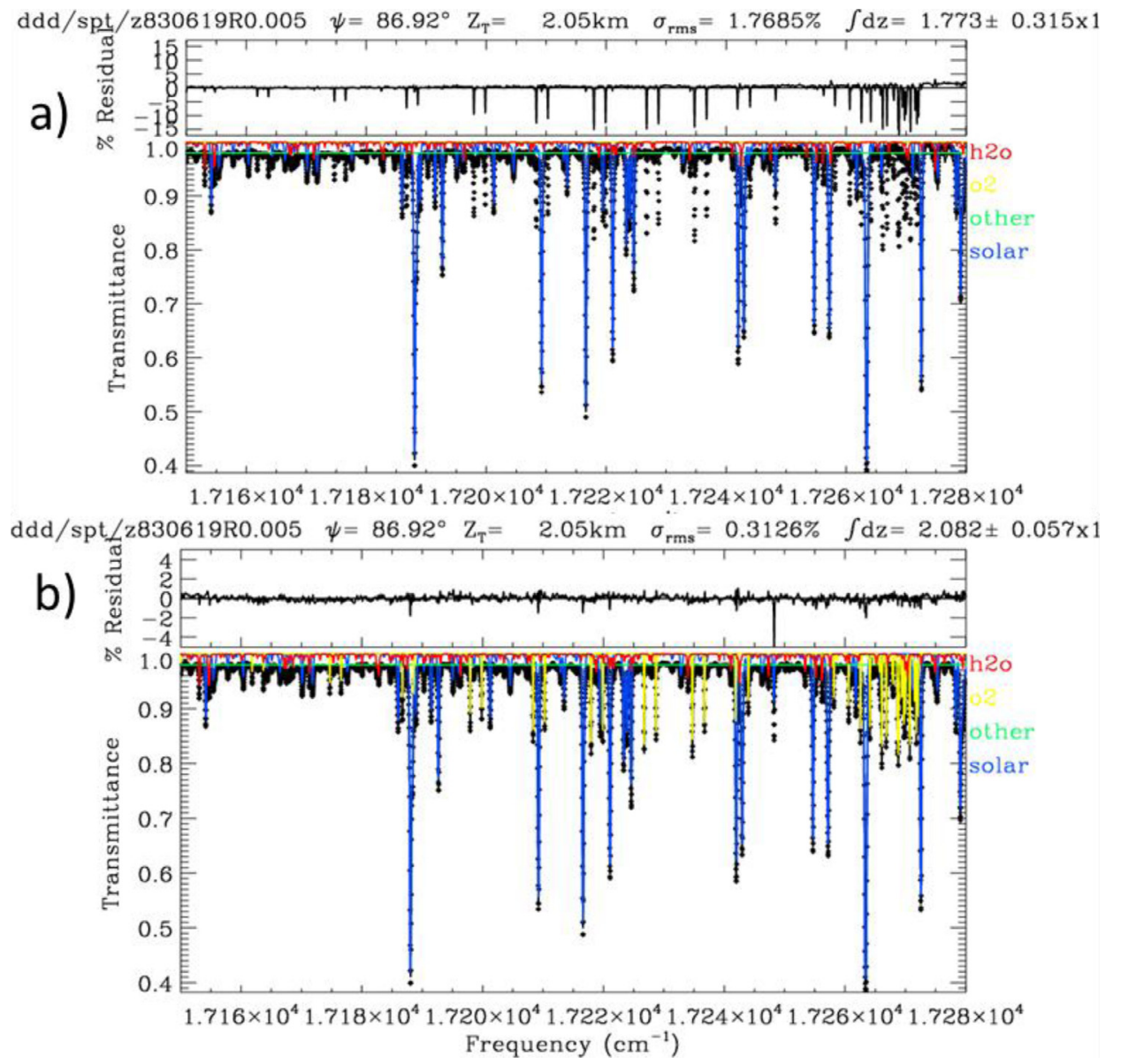

Fig. 18. High-air mass ground-based Kitt Peak spectrum fitted without (a) and with (b) $b^{1} \Sigma_{g}^{+}(\mathrm{v}=3)-X^{3} \Sigma_{g}^{-}(\mathrm{v}=0)$ band of $\mathrm{O}_{2}$.

band were estimated using the approach described in Gordon et al. [259]. Fig. 18b shows that the inclusion of this band makes the difference and it was included into HITRAN2016.

In a similar fashion it was discovered that it is important to include the $b^{1} \sum_{g}^{+}(\mathrm{v}=2)-X^{3} \sum_{g}^{-}(\mathrm{v}=1)$ band (basically a hot band underlying the $B$-band). Intensities for this band were also determined based on atmospheric spectra.

Finally, the UV bands of oxygen are not stored separately anymore and one can retrieve them from www.hitran.org in the same manner as the other bands.

\subsubsection{B-band}

Recently Domysławska et al. [260] developed a dataset of the line-shape parameters for the $\mathrm{B}$-band of self-broadened $\mathrm{O}_{2}$ (the main isotopologue), based on accurate CRDS measurements [261,262]. The dataset includes 49 lines from the P and R branches. It was observed that, at the considered level of signal-to-noise ratio, the spectra are well reproduced by the speed-dependent Voigt profile with quadratic speed dependence. In the near future we plan to incorporate these data into HITRAN within the formalism of the Hartmann-Tran profile by setting the frequency of the velocity-changing collisions, $v_{\mathrm{Vc}}$, and the correlation parameter, $\eta$, to zero.

\subsection{NO (molecule 8)}

Nitric oxide is an important constituent of the terrestrial atmosphere, where it is commonly detected and quantified by its fundamental IR band. The rotational spectrum plays an important role for the detection in diverse interstellar sources, but may also be used for detection in the Earth's upper atmosphere; Müller et al. [263] give a brief overview with appropriate references.

Positions and intensities for all stable NO isotopologues and for the first excited state in the pure rotation transition (1-1) of the principal isotopologue were based on Müller et al. [263] who performed a combined fit of all available rotational data together with heterodyne IR data of the fundamental band of the main species. All experimental data were carefully evaluated, a few poorly-fitting transition frequencies were omitted, and the uncertainties of some additional data were slightly adjusted as detailed in Ref. [263].

The ${ }^{14} \mathrm{~N}^{16} \mathrm{O}$ line position and intensity data consist of millimeter and lower submillimeter data from Pickett et al. [264] and tunable far-IR data from Varberg et al. [265] in $\mathrm{v}=0$. The $\Lambda$-doubling transitions in $\mathrm{v}=0$ were mainly taken from Meerts and Dymanus [266]; additional data were taken from Refs. [267,268]. Further $\Lambda$ doubling transitions in $\mathrm{v}=0$ and 1 were taken from Dale et al. [269] and Lowe et al. [270]. Also used in the fit were the $\mathrm{v}=1$ 0 heterodyne IR data from Hinz et al. [271] and the very accurate data from Saupe et al. [272].

Millimeter and lower submillimeter data of ${ }^{15} \mathrm{~N}^{16} \mathrm{O}$ were contributed by Saleck et al. [273], and tunable far-IR data by Varberg et al. [265]. The $\Lambda$-doubling transitions were taken from Meerts and Dymanus [266]. Millimeter and lower submillimeter data of the isotopologue ${ }^{14} \mathrm{~N}^{18} \mathrm{O}$ were also published by Saleck et al. [273], and tunable far-IR data by Müller et al. [263]. Finally, millimeter data of ${ }^{14} \mathrm{~N}^{17} \mathrm{O}$ and ${ }^{15} \mathrm{~N}^{18} \mathrm{O}$ were taken from Saleck et al. [274].

A very accurate value of the ground-state electric dipole moment of ${ }^{14} \mathrm{~N}^{16} \mathrm{O}$ was published in Ref. [268]; a value for $\mathrm{v}=1$ was determined in Ref. [275]. The strength of the magnetic dipole 
transition was derived from the electron spin-rotation parameter $\gamma$ employing Curl's relationship [276] to evaluate the $g$-factor of the respective isotopologue and in the appropriate vibrational state.

The data set has been extended considerably by FTIR data of ${ }^{14} \mathrm{~N}^{16} \mathrm{O}$ reaching high $\mathrm{v}$ and by FTIR data of minor isotopic species from Ref. [277]. This will be used for future editions of HITRAN and HITEMP [278]. The spectroscopic model developed in this study has also been used to compute $g$-factors for the system [279].

\section{9. $\mathrm{SO}_{2}$ (molecule 9)}

Since the 2008 edition of HITRAN, the air-broadening coefficients of sulfur dioxide have been a constant, $\gamma_{\text {air }}=0.1025 \mathrm{~cm}^{-1}$, for line positions lower than $3000 \mathrm{~cm}^{-1}$; with $\gamma_{\text {air }}=0.1 \mathrm{~cm}^{-1}$ for line positions larger than $3000 \mathrm{~cm}^{-1}$ (see HITRAN2008 paper [198] for details). Recently, new measurements have been made of the air-broadening coefficients of $\mathrm{SO}_{2}$ from infrared and submillimeter-wave spectra [280,281]. When analyzing these data together with previously available experiments [282-285], a slow decrease of the air-broadening coefficients with increasing $\left(J^{\prime \prime}+0.2 K_{a}^{\prime \prime}\right)$ lower-state quantum number combination was observed (see the report by Tan et al. [286] for details). The vibrational dependence of the broadening parameters is demonstrated to be very small or negligible.

A linear extrapolation (see Eq. (1)) up to $\left(\mathrm{J}^{\prime \prime}+0.2 \mathrm{Ka}^{\prime \prime}\right) \leq 62$ (with error code $4(10-20 \%)$ ) was used, while for the transitions with $\left(\mathrm{J}^{\prime \prime}+0.2 \mathrm{Ka}^{\prime \prime}\right)>62$, the value corresponding to the experimental value with the largest $\left(\mathrm{J}^{\prime \prime}+0.2 \mathrm{Ka}^{\prime \prime}\right)$ was used.

$\gamma_{\text {air }}=0.10731-2.88311 \times 10^{-4}\left(J^{\prime \prime}+0.2 K_{a}^{\prime \prime}\right)$

A complete line-by-line database of room temperature selfbroadening coefficients for about $1650 \mathrm{SO}_{2}$ transitions has been compiled by Tasinato et al. [287]. These data are used to update the self-broadening coefficients for the corresponding transitions. The error code has been set to 5 , since we estimate the accuracy to be $5-6 \%$. We also use the average values from Ref. [287] (with error code 4 ) of the same quantum numbers $\left(J^{\prime \prime}, K_{a}{ }^{\prime \prime}\right)$ for all the other transitions with corresponding rotational quantum numbers. More details about this update of air and self-broadening parameters can be found in the report by Tan et al. [286].

Another update to the $\mathrm{SO}_{2}$ data is the addition of pressure shifts, line widths and their temperature-dependence exponents in $\mathrm{H}_{2}$, He and $\mathrm{CO}_{2}$ environments for every HITRAN transition. This update is described in detail by Wilzewski et al. [4]. While shifts of the $\mathrm{SO}_{2}$ lines due to $\mathrm{H}_{2}$, He or $\mathrm{CO}_{2}$ were set to the default value of zero for every line because no reports of these parameters exist in available peer-reviewed sources, broadening parameters and their temperature exponents have been added based on Refs. [280,281,284,288,289]. Especially in the case of the $\mathrm{SO}_{2}-\mathrm{CO}_{2}$ system, the amount of available data used to construct the line list of broadening parameters is minimal, and future measurements of the $\mathrm{SO}_{2}-\mathrm{H}_{2}$, - $\mathrm{He}$ and $-\mathrm{CO}_{2}$ systems would help to improve the present semi-empirical parameter sets.

There has been significant recent progress in the computation of extensive, accurate theoretical line lists for both room temperature [290,291] and hot [292] $\mathrm{SO}_{2}$. The intensities in these line lists are accurate enough to provide alternatives for missing bands of both the main and the minor isotopologues; this should be considered in future updates. They can be supplemented with line positions calculated using constants from recent works from Ulenikov et al. [293-297] and references therein.

\subsection{0. $\mathrm{NO}_{2}$ (molecule 10)}

Unchanged.

\subsection{1. $\mathrm{NH}_{3}$ (molecule 11)}

$\mathrm{NH}_{3}$ is a very important constituent of the atmospheres of giant planets and small, cold stars. It is also abundant in various environments of the interstellar medium and occurs in the terrestrial atmosphere. The isotopologue with ${ }^{15} \mathrm{~N}$ can be very important for studying isotopic fractionation or for studying the opacity of lines pertaining to ${ }^{14} \mathrm{NH}_{3}$.

Positions and intensities of the ${ }^{15} \mathrm{NH}_{3}$ pure-tunneling and rotation-tunneling transitions were taken from the CDMS. It is based on rotation-tunneling transition frequencies from Belov et al. [298] and Winnewisser et al. [299], on pure tunneling data from Kukolich [300], in part corrected by Hougen [301], and from Sasada [302]. Also included are FIR data from Urban et al. [303] and Carlotti et al. [304]. Initial spectroscopic parameters were taken from a modified fit of ${ }^{14} \mathrm{NH}_{3}$ data by Chen et al. [305]. Quantum chemical calculations and trial fits suggested that the $C$ rotational constant cannot be determined reliably from these data. Therefore, its value was kept fixed to the ${ }^{14} \mathrm{NH}_{3}$ value derived from Ref. [306] modified by the ${ }^{14} \mathrm{NH}_{3} /{ }^{15} \mathrm{NH}_{3}$ difference calculated from energies calculated by Huang et al. [306]. The dipole moment and its first order $J$ and $K$ distortion corrections were taken from Tanaka et al. [307]. The rotation-tunneling transitions extend to $J=14$ [304], and the pure tunneling transitions [302] even higher in $J$. The predictions should show FIR accuracy at least up to $J=14$, but predictions higher than $J=15$ should be viewed with increasing caution. Moreover, frequencies and possibly also intensities of the weak $\Delta K=3$ transitions should be viewed with some caution.

HITRAN2012 only contained ammonia transitions up to $7000 \mathrm{~cm}^{-1}$. Recently, Barton et al. [308] analyzed Fourier Transform (FT) absorption spectra recorded at the National Solar Observatory by C. De Bergh in 1980, providing partially-assigned lists of measured line positions and intensities. The present update includes 8468 lines in the region $7400-8640 \mathrm{~cm}^{-1}$ [308]; 2474 of these lines are fully assigned using the quantum numbers recommended by Down et al. [309]. Barton et al. [310] have also analyzed and partially assigned a further spectrum covering the 8800 to $10,400 \mathrm{~cm}^{-1}$ region. These data were also included in HITRAN2016.

At lower wavenumbers, comparisons between the HITRAN data and comprehensive variational line lists $[311,312]$ give good agreement below $4000 \mathrm{~cm}^{-1}$. However, comparisons with these line lists suggest that the current HITRAN compilation is missing a significant number of lines in the $4000-7000 \mathrm{~cm}^{-1}$ region. Attempts to resolve this problem using a combination of available variational line lists referred to as BYTe [311,312] and empirical energy levels provided using the MARVEL procedure [313] to give a hybrid line list referred to as "BARVEL" proved to be inferior to HITRAN2012 when compared with cross sections from PNNL [314]. It would appear that further work is needed on both the computed line list and the MARVEL dataset in this region. Work in both these directions is currently being undertaken and, in particular, a new highaccuracy $a b$ initio potential energy surface [315] should provide a much better starting point for improved nuclear motion calculations.

Pressure-broadening coefficients and their temperature dependence exponent were calculated from the polynomial coefficients of Nemtchinov et al. [316] derived from the $v_{2}$ band measurements. Nemtchinov et al. suggested that the rotational dependence of the air- and self-broadening half width, $\gamma_{\text {air }}$ and $\gamma_{\text {self, can be }}$ represented using the following polynomial:

$\gamma^{0}(m, K)=\beta_{0}+\beta_{1} m+\beta_{2} K+\beta_{3} m^{2}+\beta_{4} K^{2}+\beta_{5} m K$

where $m=|-J, J, J+1|$ for the $\mathrm{P}, \mathrm{Q}$ and $\mathrm{R}$ branches, and $\beta_{i}$ are the polynomial coefficients. This polynomial was used only for $J \leq 8$ with error code 6 for both $\gamma_{\text {air }}$ and $\gamma_{\text {self }}$, and for the other transitions with, $J>8$ the extrapolated value corresponding to the 

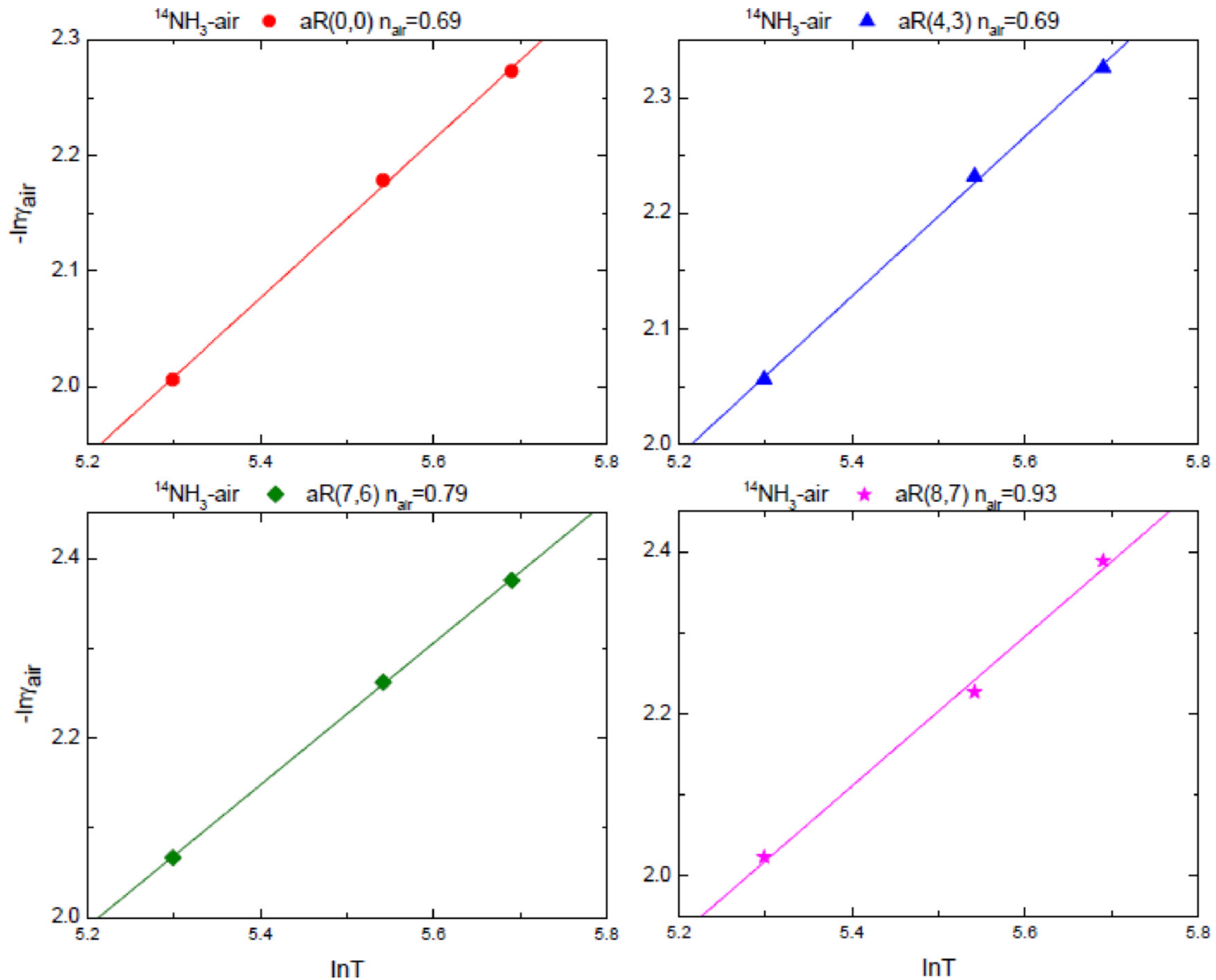

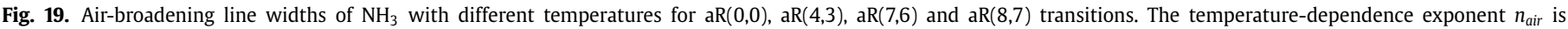
determined from the slope of the least-squares fits of $-\ln \gamma_{\text {air }}^{0}(T) V S . \ln T$.

largest $J$ and $K$ was used. For instance, when $J>8$ the default values $\gamma_{\text {air }}=0.0906 \mathrm{~cm}^{-1} / \mathrm{atm}$ and $\gamma_{\text {self }}=0.5 \mathrm{~cm}^{-1} / \mathrm{atm}$ have been used for all these transitions, and the error code has been set to 5 for both $\gamma_{\text {air }}$ and $\gamma_{\text {self. }}$

The temperature dependence exponents have also been measured in the $v_{2}$ band by Nemtchinov et al. [316] but for broadening by $\mathrm{O}_{2}$ and $\mathrm{N}_{2}$ separately. We note that in the literature many researchers determine the temperature exponent to be

$\boldsymbol{n}_{\text {air }}=0.79 \boldsymbol{n}_{\mathrm{N} 2}+0.21 \boldsymbol{n}_{02}$

by analogy with the standard approximation

$\gamma_{\mathrm{air}}=0.79 \gamma_{\mathrm{N} 2}+0.21 \gamma_{\mathrm{O} 2}$

We note however that Eq. (3) and Eq. (4) contradict each other because the variation of the air-broadening coefficients with temperature $\mathrm{T}$ is given by:

$\frac{\gamma_{\text {air }}(T)}{\gamma_{\text {air }}\left(T_{\text {ref }}\right)}=\left(\frac{T_{\text {ref }}}{T}\right)^{n_{\text {air }}}$

Here $T_{\text {ref }}$ is the reference temperature. Then we have:

$n_{\text {air }}=-\frac{\ln \gamma_{a i r}\left(T_{r e f}\right)-\ln \gamma_{a i r}(T)}{\ln T_{\text {ref }}-\ln T}$,

where $\gamma_{\text {air }}$ can be defined using Eq (4). Therefore the measured half widths induced by $\mathrm{O}_{2}$ and $\mathrm{N}_{2}$ at 200,255 and $296 \mathrm{~K}$ can be determined from the values of $n_{\text {air }}$ as the slope of the least-squares fits of $-\ln \gamma_{\text {air }}^{0}(T) v s \ln T$, as shown in Fig. 19.
The broadening algorithm described above as well as the temperature dependence procedure is also used throughout the entire database for both isotopologues ${ }^{14} \mathrm{NH}_{3}$ and ${ }^{15} \mathrm{NH}_{3}$.

Semi-empirical line widths, pressure shifts and temperaturedependence exponents of ammonia perturbed by $\mathrm{H}_{2}$, $\mathrm{He}$ and $\mathrm{CO}_{2}$ have been added to the database for the first time based on the algorithm described in Wilzewski et al. [4]. This algorithm makes use of the results from Refs. [316-320] (for $\mathrm{H}_{2}$ ), Refs. [317,319-325] (for $\mathrm{He}$ ) and Refs. [321,326,327] (for $\mathrm{CO}_{2}$ ). Even though the new parameters were constructed from a relatively large number of datareporting articles, the database would profit from future studies of $\mathrm{NH}_{3}$ absorption lines perturbed by $\mathrm{H}_{2}$, He and $\mathrm{CO}_{2}$ for validation purposes as described in Ref. [4].

\subsection{2. $\mathrm{HNO}_{3}$ (molecule 12)}

For nitric acid $\left(\mathrm{HNO}_{3}\right)$, the three strongest absorption band systems, namely the $\left\{v_{5}, 2 v_{9}\right\},\left\{v_{3}, v_{4}\right\}$ interacting bands, and the $v_{2}$ band, are located at $11,7.6$ and $5.8 \mu \mathrm{m}$ respectively. Although two times weaker than the bands observed in the two other ranges, the $11-\mu \mathrm{m}$ band system is of particular atmospheric interest since it coincides with a rather clear atmospheric window. As the HITRAN2012 database [1] provides a reliable description of the nitric acid spectrum at $11 \mu \mathrm{m}$ (the line list includes the $v_{5}$ and $2 v_{9}$ cold bands for the $\mathrm{H}^{14} \mathrm{~N}^{16} \mathrm{O}_{3}$ and $\mathrm{H}^{15} \mathrm{~N}^{16} \mathrm{O}_{3}$ isotopologues, as well as the $v_{5}+v_{9}-v_{9}, 3 v_{9}-v_{9}, v_{5}+v_{7}-v_{7}$ and $v_{5}+v_{6}-v_{6}$ hot bands of $\mathrm{H}^{14} \mathrm{~N}^{16} \mathrm{O}_{3}$ ), this region is used for $\mathrm{HNO}_{3}$ retrievals by numerous satellite or balloon-borne instruments [328-333]. 
Table 8

Comparison of the $\mathrm{HNO}_{3}$ line parameters in the 7.6- $\mu \mathrm{m}$ region in the 2012 and 2016 editions of HITRAN.

\begin{tabular}{|c|c|c|c|c|c|c|}
\hline \multicolumn{7}{|c|}{ (a) HITRAN2012 } \\
\hline Band & $N$ & $S_{\text {tot }}\left(10^{-18}\right)$ & $v_{\min }$ & $v_{\max }$ & $S_{\min }\left(10^{-23}\right)$ & $S_{\max }\left(10^{-21}\right)$ \\
\hline$v_{3}$ & 21,308 & 25.37 & 1098.376 & 1387.849 & 1.037 & 31.33 \\
\hline$v_{4}$ & 19,584 & 12.78 & 1229.867 & 1387.561 & 1.037 & 18.67 \\
\hline Sum & & 38.15 & & & & \\
\hline
\end{tabular}

(b) HITRAN2016

\begin{tabular}{lllllll}
\hline Band & $N$ & $S_{\text {tot }}\left(10^{-18}\right)$ & $v_{\min }$ & $v_{\max }$ & $S_{\min }\left(10^{-25}\right)$ & $S_{\max }\left(10^{-21}\right)$ \\
\hline$v_{3}$ & 16,408 & 24.94 & 1252.010 & 1394.177 & 4.910 & 32.0 \\
$v_{4}$ & 18,105 & 9.834 & 1238.929 & 1387.081 & 4.020 & 10.7 \\
$2 v_{6}$ & 2451 & 0.1194 & 1243.465 & 1348.275 & 4.624 & 3.660 \\
$v_{5}+v_{9}$ & 13,817 & 0.7163 & 1246.929 & 1390.071 & 2.081 & 3.543 \\
$v_{7}+v_{8}$ & 11,125 & 0.7615 & 1246.422 & 1395.679 & 2.314 & 5.017 \\
$3 v_{9}$ & 13,894 & 1.177 & 1233.107 & 1388.497 & 4.582 & 2.378 \\
Sum & & 37.55 & & & & \\
$v_{3}+v_{9}-v_{9}$ & 12,106 & 1.408 & 1271.050 & 1394.899 & 5.285 & 1.798 \\
\hline
\end{tabular}

Note: $N$ is the number of lines, $v_{\min }$ and $v_{\max }\left(\mathrm{cm}^{-1}\right)$ are the lower and upper ranges of the band, $S_{\min }$ and $S_{\max }$ are the smallest and largest line intensity $\left(\mathrm{cm}^{-1} /\right.$ \{molecule $\mathrm{cm}^{-2}$ \} at $296 \mathrm{~K}$ ) and $S_{\text {tot }}$ is the sum of the line intensities.

However, retrievals of nitric acid at altitudes higher than $\sim 35 \mathrm{~km}$, where it is less abundant, require use of the stronger infrared signatures at 5.8 or $7.6 \mu \mathrm{m}$. Because the first spectral region overlaps rather strongly with water vapor absorption in atmospheric spectra, the 7.6- $\mu \mathrm{m}$ band system is preferable. However, most satellite or balloon-borne instruments avoided using this latter region up to now for $\mathrm{HNO}_{3}$ retrievals because of the poor quality of the available $\mathrm{HNO}_{3}$ line positions and line intensities.

For $\mathrm{HNO}_{3}$, the 7.6- $\mu \mathrm{m}$ spectral region in HITRAN2012 is limited to the $v_{3}$ and $v_{4}$ cold bands of the main isotopologue, $\mathrm{H}^{14} \mathrm{~N}^{16} \mathrm{O}_{3}$. In addition, the quality of the line positions and intensities available therein is rather poor, mainly because the theoretical model used at that time [334] accounted only for the interactions coupling energy levels belonging to the $v_{3}$ and $v_{4}$ bright states but was neglecting interactions with several dark states present in the same energy range.

Therefore it was decided to update the $\mathrm{HNO}_{3}$ line list at $7.6 \mu \mathrm{m}$. First, a complete reinvestigation of the line positions for nitric acid at $7.6 \mu \mathrm{m}$ was performed [335]. In contrast to the previous analysis [334], the new Hamiltonian model accounted properly for the various vibration-rotation resonances and torsional effects affecting the $v_{3}$ and $v_{4}$ bright states and the four dark states $2 v_{6}, 3 v_{9}, v_{5}+$ $v_{9}$ and $v_{7}+v_{8}$. Additionally, the $v_{3}+v_{9}-v_{9}$ hot band was identified for the first time [335].

At the end of this first study [335], a new list of line positions and of preliminary line intensities was generated. This list included contribution from the $v_{3}$ and $v_{4}$ cold bands, the $2 v_{6}$, $3 v_{9}, v_{5}+v_{9}, v_{7}+v_{8}$ dark bands, and the $v_{3}+v_{9}-v_{9}$ hot band of the main isotopologue. The final line intensities at $7.6 \mu \mathrm{m}$ were generated through a calibration process during which the $\mathrm{HNO}_{3}$ vol mixing ratio profiles retrieved from the "Michelson Interferometer for Passive Atmospheric Sounding" (MIPAS) limb emission radiances in the 11 - and 7.6- $\mu \mathrm{m}$ domains were compared. This line list at $7.6 \mu \mathrm{m}$, which was also validated using the available laboratory information (individual line intensities and absorption cross sections), proved to provide an improved description of the 7.6- $\mu \mathrm{m}$ region absorption of nitric acid [336].

Table 8 compares the $\mathrm{HNO}_{3}$ line lists at $7.6 \mu \mathrm{m}$ given in the 2012 and 2016 releases of HITRAN showing significant changes. Finally, Fig. 20 compares the observed spectrum in the 7.6- $\mu \mathrm{m}$ spectral region (laboratory spectrum recorded in Giessen) to synthetic spectra generated using HITRAN2012 and HITRAN2016 showing the improvements brought by the new data as shown by the resid-

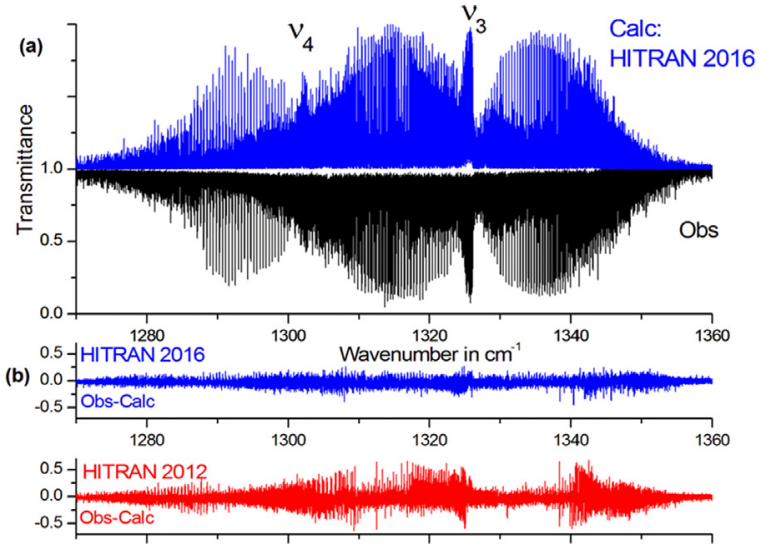

Fig. 20. Overview of the Fourier transform spectrum recorded at high resolution in Giessen University in 2004 [335] and comparisons with the models performed using the HITRAN2012 and HITRAN2016 editions.

uals. In conclusion, it is expected that this study should help to improve $\mathrm{HNO}_{3}$ satellite retrievals by allowing measurements to be performed simultaneously in the 11 - and $7.6-\mu \mathrm{m}$ spectral regions.

In the HITRAN2008 paper, the several-item roadmap for the improvements to the nitric acid spectroscopy was presented. A majority of these problems have now been solved. However, the biggest challenge still remains, the lack of a line-by-line list for the $v_{1}$ band of $\mathrm{HNO}_{3}$ centered at $3551 \mathrm{~cm}^{-1}$. It is a pronounced feature that interferes with other gases absorbing at these wavenumbers. Fig. 5 in the paper by Toon et al. [147] shows how the lack of spectroscopic parameters negatively affects retrievals from the Mk IV balloon observations. Low-resolution spectra of this band exist in the PNNL database [314] but at a limited set of thermodynamic conditions, while $a b$ initio calculations for this molecule $[337,338]$ do not yet allow accurate atmospheric retrievals.

\subsection{3. $\mathrm{OH}$ (molecule 13)}

The line list for the hydroxyl radical remained unchanged. The recent study by Brooke et al. [339] could be considered for updating intensities of the weak lines (this is more relevant for HITEMP [278]) in the near future. 


\subsection{HF (molecule 14)}

Transitions of HF with changes in vibrational quantum number, $\Delta v$, larger than 10 were removed from the database along with transitions with $\Delta \mathrm{v}>8$ for DF. This removal was carried out because there were numerical issues in calculating the intensities of these transitions. Medvedev et al. [340] explain how the use of double precision in calculating overtone intensities may lead to numerical errors and that quadruple precision is needed. Additional numerical errors occur depending on the interpolation techniques used for the dipole moment functions as explained in Medvedev et al. [341].

Broadening, shifting and temperature-dependence parameters of $\mathrm{HF}$ due to the pressure of $\mathrm{H}_{2}$, He and $\mathrm{CO}_{2}$ have been added to HITRAN for the first time. The semi-empirical procedure described in Wilzewski et al. [4] was developed using results from Ref. [342] (for $\mathrm{H}_{2}$ ), Refs. [343-345] (for $\mathrm{CO}_{2}$ ) and Refs. [346-348] (for $\mathrm{He}$ ). To expand and validate the present parameters, more measurements are needed, while it would also be valuable to have more dedicated data for DF.

\subsection{5. $\mathrm{HCl}$ (molecule 15)}

The intensity issue mentioned above for HF concerning the removal of high overtones of all isotopologues was likewise applied to $\mathrm{HCl}$ (i.e. transitions with $\Delta \mathrm{v}>8$ were removed for all four isotopologues of hydrogen chloride).

Ref. [4] describes in detail the addition of $\mathrm{H}_{2-}$, $\mathrm{He}-$ and $\mathrm{CO}_{2}-$ broadening, -shifting and temperature-dependence parameters of $\mathrm{HCl}$ to the database. Semi-empirical $\mathrm{HCl}-\mathrm{CO}_{2}$ broadening and shifting parameters for each HITRAN transition are based on Refs. [345,349] and sophisticated extrapolations of these results, while Refs. [350,351] were used to construct $\mathrm{HCl}-\mathrm{He}$ linewidths and shifts. For the $\mathrm{HCl}-\mathrm{H}_{2}$ system, the new parameters are based on Refs. [352-355]. In the future, measurements of the temperaturedependence of foreign-broadened $\mathrm{HCl}$ spectral lines will be very helpful additions to the database.

\subsection{6. $\mathrm{HBr}$ (molecule 16)}

Unchanged.

\subsection{HI (molecule 17)}

Unchanged.

\subsection{8. $\mathrm{ClO}$ (molecule 18)}

Unchanged.

\subsection{OCS (molecule 19)}

Carbonyl sulfide is the most abundant sulfur-containing species in the free troposphere, often monitored using optical remote sensing in the $5-\mu \mathrm{m}$ region [356]. OCS is present in the atmosphere of Venus [357]. The increasing interest for the application of very sensitive cavity ring down spectroscopy to the in situ detection of trace species [358] makes the near infrared range more and more attractive. However, the HITRAN2012 database provided spectroscopic information for OCS only up to $4200 \mathrm{~cm}^{-1}$ [1]. Recently, Fourier transform spectra of carbonyl sulfide were recorded between 6170 and $6680 \mathrm{~cm}^{-1}$ and between 7700 and $8160 \mathrm{~cm}^{-1}$ using a femto/OPO laser absorption source and cavity enhanced spectroscopy [359]. The strongest bands observed in these two regions are $3 v_{1}+2 v_{3}$ (near $6640 \mathrm{~cm}^{-1}$ ) and $2 v_{1}+3 v_{3}$ (near $7812 \mathrm{~cm}^{-1}$ ), respectively. The line positions thus measured (with an accuracy estimated to be $0.001 \mathrm{~cm}^{-1}$ for not-too-weak unblended lines) were used to update a global polyad model of carbonyl sulfide (see Ref. [360] and references therein).

This global polyad model was then used to generate a list of line positions, assignments, lower-state energies, and relative intensities for the main isotopologue. Because quantitative intensity information could not be retrieved from the measured spectra, the predicted relative intensities had to be scaled relying on additional information. A scaling factor was determined for each of the two spectral regions by matching calculated spectra of the $3 v_{1}+2 v_{3}$ and $2 v_{1}+3 v_{3}$ bands to their Fourier transform spectra, recorded previously [360]. Because predicted relative intensities only make sense for transitions involving levels belonging to the same polyads, these two scaling factors could only be applied to bands corresponding to transitions involving upper vibrational levels belonging to the same polyads as the upper levels of the $3 v_{1}+2 v_{3}$ and $2 v_{1}+3 v_{3}$ bands. Line intensities could therefore be obtained for cold bands matching this constraint, and hot bands involving variations of the vibrational quantum numbers identical to these cold bands (thus assuming that the vibrational dependence of the transition moments is negligible). Because isotopic substitution is not expected to significantly change the transition moments, line intensities were also obtained for the same bands belonging to ${ }^{16} \mathrm{O}^{12} \mathrm{C}^{34} \mathrm{~S},{ }^{16} \mathrm{O}^{13} \mathrm{C}^{32} \mathrm{~S}$ and ${ }^{16} \mathrm{O}^{12} \mathrm{C}^{33} \mathrm{~S}$. All these predicted bands cover the ranges 6484-6650 $\mathrm{cm}^{-1}$ (region A) and 7728$7821 \mathrm{~cm}^{-1}$ (region B). These two regions, involving a total of 3660 predicted lines, are presented in Figs. 13 and 14 of Ref. [359]. Note that only lines having intensities larger than $3.0 \times 10^{-28}$ (region A) and $6.7 \times 10^{-28}$ (region B) $\mathrm{cm}^{-1} /\left(\right.$ molecule $\left.\mathrm{cm}^{-2}\right)$ at $296 \mathrm{~K}$ were considered. These intensity thresholds are slightly lower than the experimental limits. The supplementary material of Ref. [359] also includes a list of line positions, assignments, and lower-state energies for 3417 additional lines of ${ }^{16} \mathrm{O}^{12} \mathrm{C}^{32} \mathrm{~S},{ }^{16} \mathrm{O}^{12} \mathrm{C}^{34} \mathrm{~S},{ }^{16} \mathrm{O}^{13} \mathrm{C}^{32} \mathrm{~S}$ and ${ }^{16} \mathrm{O}^{12} \mathrm{C}^{33} \mathrm{~S}$, covering the $6200-6449 \mathrm{~cm}^{-1}$ and $7901-8120 \mathrm{~cm}^{-1}$ ranges. Because of the constraint mentioned above, the relative intensities of these lines could not be scaled and were set to zero, and they were not included in HITRAN2016.

The spectroscopic information included in the new edition of HITRAN is the data of regions $A$ and $B$ obtained as described above, to which 10 lines belonging to the $2 v_{1}+2 v_{2}{ }^{\circ}+2 v_{3}$ band of the ${ }^{16} \mathrm{O}^{13} \mathrm{C}^{32} \mathrm{~S}$ isotopologue (between 6650 and $6660 \mathrm{~cm}^{-1}$ ) were added. The origin of this band is located near $6650.773 \mathrm{~cm}^{-1}$. Relying on predictions of the global polyad model, which turned out to be as close as a few $10^{-3} \mathrm{~cm}^{-1}$ to the observed positions, 17 $\mathrm{P}$ and $\mathrm{R}$ branch lines of the $2 v_{1}+2 v_{2}{ }^{\circ}+2 v_{3}$ band of ${ }^{16} \mathrm{O}^{13} \mathrm{C}^{32} \mathrm{~S}$ could be observed, their positions measured in the spectra of Ref. [359] and included in the global modeling to yield improved predictions. These improved predictions are included in this edition of HITRAN. Note that the upper level of this band is the highest ever observed for ${ }^{16} \mathrm{O}^{13} \mathrm{C}^{32} \mathrm{~S}$. In the supplementary material of Ref. [359], 152 pairs of upper vibrational levels have identical assignments as a result of the combined effects of heavy mixing near avoided crossings and the assignments of the levels being based on the largest coefficient of their eigenvectors. To remove these duplicate assignments, one vibrational level in each pair was manually reassigned through careful examination of the corresponding energy level structures. Additionally, it was found that the line intensities provided in the supplementary material of Ref. [359] could be affected by the number of significant digits used when storing intermediate results. The line intensities provided in this edition of HITRAN correct this problem and are listed with the appropriate number of significant digits. The HITRAN line list for OCS contains self- and air-broadening coefficients for all the lines calculated using the Padé approximant proposed of Koshelev and Tretyakov [361].

$\mathrm{H}_{2}$-, $\mathrm{He}$ - and $\mathrm{CO}_{2}$-broadened linewidths of OCS were added to HITRAN for the first time based on semi-empirical models derived 
in Wilzewski et al. [4]. The following experimental data were used to derive these models: Ref. [362] (for $\mathrm{H}_{2}$ ), Refs. [362-365] (for He) and Ref. [366] (for $\mathrm{CO}_{2}$ ). There are no lineshifts and temperaturedependence parameters due to $\mathrm{H}_{2}$, He or $\mathrm{CO}_{2}$ available in the literature.

\subsection{0. $\mathrm{H}_{2} \mathrm{CO}$ (molecule 20)}

Unchanged.

\subsection{1. $\mathrm{HOCl}$ (molecule 21)}

The $v_{2}$ band region of hypochlorous acid, $\mathrm{HOCl}$, was first introduced in HITRAN2004 [3] based on the line list from Vander Auwera et al. [367]. It was found that 49 lines of the principal isotopologue of $\mathrm{HOCl}$ in HITRAN had an incorrect vibrational assignment being attributed to the $v_{2}$ band, while in reality they are $2 v_{3}$ lines. Also, the quantum rotational assignment of the line at $1237.62928 \mathrm{~cm}^{-1}$ was incorrect. All of the assignments are now fixed, based on the correct information provided in the original publication [367].

\subsection{2. $\mathrm{N}_{2}$ (molecule 22)}

It was found that the abundance of the ${ }^{14} \mathrm{~N}^{15} \mathrm{~N}$ isotopologue was incorrectly calculated in the original release of HITRAN2012; this issue with the intensities has been fixed in the latest edition.

\subsection{HCN (molecule 23)}

Hydrogen cyanide is a trace atmospheric species that can result from biomass burning [368-370]; its presence in the troposphere is being routinely monitored [371,372]. The use of HCN spectra for diagnostic applications in medicine are also being investigated [373]. HCN, and its isomer HNC (which is not yet in HITRAN), are important astronomical species with, for example, the recent tentative detection of $\mathrm{HCN}$ in the super-Earth exoplanet 55 Cancri e [374].

The spectrum of ${ }^{1} \mathrm{H}^{12} \mathrm{C}^{14} \mathrm{~N}$ at wavenumbers below $3500 \mathrm{~cm}^{-1}$ has been included in HITRAN for some time. Here we extend the data available to higher wavenumbers. These data were based on the semi-empirical line list generated by Barber et al. [375] as part of the ExoMol project [376]. For full line lists appropriate for higher temperatures and covering both HCN and HNC the reader should consult these sources directly.

HITRAN has been updated with ${ }^{1} \mathrm{H}^{12} \mathrm{C}^{14} \mathrm{~N}$ lines for wavenumbers above $3500 \mathrm{~cm}^{-1}$ and limited to transitions which have intensity greater than $10^{-29} \mathrm{~cm}^{-1} /\left(\right.$ molecule $\left.\bullet \mathrm{cm}^{-2}\right)$; these criteria give a total of 55,153 lines in that spectral region. Wavenumbers for the majority of these lines were taken from the empirical energy levels of Mellau [377,378]. However, transition intensities are ones computed by Harris et al. [379] using the $a b$ initio dipole moment and potential energy surface of Van Mourik et al. [380]. The Harris et al. calculations are not sufficiently accurate for transition frequencies but the evidence is that the transition intensities can be considered fairly reliable [381]. An overview of the HCN line list in HITRAN2016 is given in Fig. 21.

The air-broadening half widths were calculated using semiempirical expressions derived in Yang et al. [382]. Self-broadening parameters were calculated using the procedure explained in the HITRAN2004 paper [3], see Eq. (20) there and discussion around it.

\subsection{4. $\mathrm{CH}_{3} \mathrm{Cl}$ (molecule 24)}

The parameters of methyl chloride for 43,147 lines (7079 of which are new), were calculated based on a recent analy- sis [383] of Fourier transform spectra in the range of 1900$2600 \mathrm{~cm}^{-1}$. The data from the supplementary file of Ref. [383] were used in the update. The error code 4 was given for line positions and the error code 5 (5-10\%)- for line intensities, see Ref. [383] for details on uncertainties. We also recalculated the Einstein A coefficients for the lines which had changed intensities. In addition, a couple of issues that have been identified in the 3- $\mu \mathrm{m}$ region of $\mathrm{CH}_{3} \mathrm{Cl}$ in HITRAN2012 were fixed. In particular, the air-broadening temperature dependencies, $\mathrm{n}_{\mathrm{air}}$ of the lines around $3 \mu \mathrm{m}$ used to be negative while they are supposed to be positive. And all of the lines in the $3-\mu \mathrm{m}$ band have a shift of $-0.02 \mathrm{~cm}^{-1} / \mathrm{atm}$. We compared the calculated spectra from the HITRAN database with the PNNL database [314] and the results show that such a strong shift of the lines appears to be incorrect. Therefore the shifts were set to be zero and $\mathrm{n}_{\text {air }}$ to be positive around 3 $\mu \mathrm{m}$. In a similar fashion we removed the shift of $-0.02 \mathrm{~cm}^{-1} / \mathrm{atm}$ in the $\nu_{2}+v_{6}$ band around $5 \mu \mathrm{m}$.

Both experimental measurements and calculations have been made of the self- and air- broadening coefficients of $\mathrm{CH}_{3} \mathrm{Cl}$ recently, allowing for a relatively sophisticated update of these parameters in HITRAN. The available results have been collected and evaluated to update the database for $\mathrm{CH}_{3} \mathrm{Cl}$. The details are as follows:

For self-broadening coefficients of $\mathrm{CH}_{3} \mathrm{Cl}$ :

1. The $\mathrm{CH}_{3}{ }^{35} \mathrm{Cl}$ self-broadening coefficients at reference temperature $296 \mathrm{~K}$ for the R-branch $(\Delta K=0)$ were computed using a semi-empirical (SE) method described by Dudaryonok et al. [384]. We used these semi-empirical calculations for R-branch and P-branch (for all $\Delta K$ values) lines in all of the bands of the two isotopologues $\mathrm{CH}_{3}{ }^{35} \mathrm{Cl}$ and $\mathrm{CH}_{3}{ }^{37} \mathrm{Cl}$ considered in the database. The average value of RMS-deviations of the $\mathrm{SE} \mathrm{CH}_{3} \mathrm{Cl}$ self-broadening coefficients from the experimental data of Ref. [385] is $0.0408 \mathrm{~cm}^{-1} / \mathrm{atm}$. We used the ratio of the individual half widths to this value to estimate the uncertainties and determine a corresponding error code.

Only R-branch transitions for quantum number ranges of $0 \leq J \leq 70,0 \leq K \leq 20$ are available from Ref. [1]. The empirical relation enables evaluation of the P-branch and Q-branch broadening coefficients from those of the R-branch

$$
\gamma_{P}(J, K) \approx \gamma_{R}(J-1, K) \approx \gamma_{Q}(J, K)
$$

2. Experimental results collected from the literature were applied to corresponding transitions with the same $J$ and $K$. These experimental values supersede the calculated ones:

(a) Experimental results from Ref [386]. are used for the same isotopologue, $\left(J^{\prime}, K^{\prime}-J^{\prime \prime}, K^{\prime \prime}\right)$ transitions in all of the bands, with the error code set to 5 (5-10\%).

(b) Experimental results from Ref [385]. are for both pure rotational $(J=6,31,37,40,45,50)$ and $v_{1}$ bands of $\mathrm{CH}_{3} \mathrm{Cl}$. The measured self-broadening coefficients for pure rotational transitions are used for the corresponding transitions in the database. Experimental results for the $v_{1}$ band are used for transitions sharing the same quantum numbers throughout other bands.

For air-broadening coefficients of $\mathrm{CH}_{3} \mathrm{Cl}$ :

1. The air-broadening coefficients and associated temperature exponents for both $\mathrm{CH}_{3}{ }^{35} \mathrm{Cl}$ and $\mathrm{CH}_{3}{ }^{37} \mathrm{Cl}$ ro-vibrational transitions of R-branches $(\Delta K=0)$ are calculated by a semi-classical (SC) approach [387]. The SC calculation are used for R-branch and P-branch (for all $\Delta K$ values) lines in all of the bands assuming $\gamma_{P}(J, K) \approx \gamma_{R}(J-1, K)$.

2. Experimental results from Ref. [388] and Ref. [389] are used for the transitions sharing the same rotational quantum numbers. 


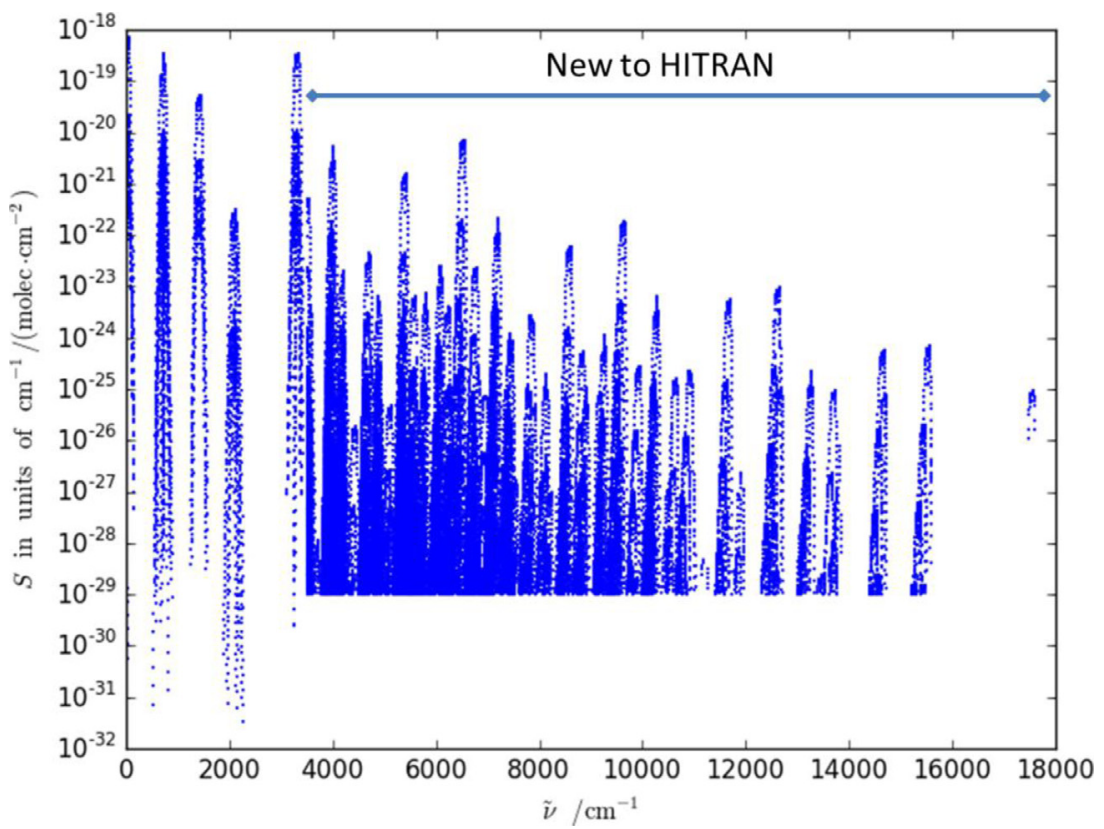

Fig. 21. Overview of the transitions of HCN in HITRAN2016 with new bands revealed.

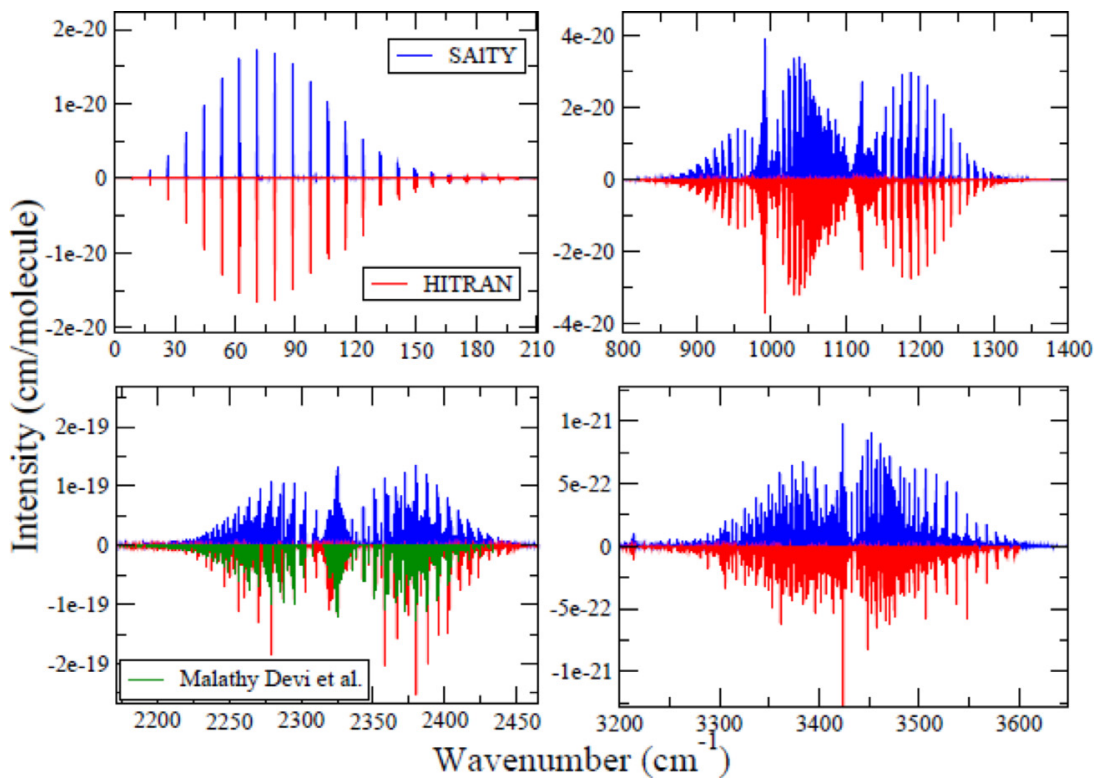

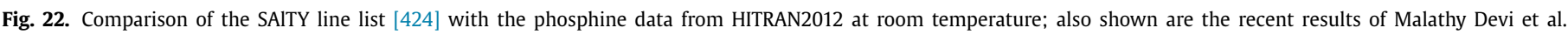
[426].

For air-broadening temperature dependencies of $\mathrm{CH}_{3} \mathrm{Cl}$ :

The air-broadening temperature exponents have been updated using the SC calculations of Buldyreva [387]. Only the rovibrational transitions of R-branches and P-branches were calculated. The values for Q-branches were kept at the constant value of 0.70 for $n_{P}(J, K) \approx n_{R}(J-1, K)$.

\subsection{5. $\mathrm{H}_{2} \mathrm{O}_{2}$ (molecule 25)}

For hydrogen peroxide, $\mathrm{H}_{2} \mathrm{O}_{2}$, the measurements of the airbroadening half widths from Refs. [390-392] were included for the corresponding transitions. The majority of the air-broadened coef- ficients in the database still have the same value of $0.1 \mathrm{~cm}^{-1} / \mathrm{atm}$ estimated from the measurements of Malathy Devi et al. [392].

Currently the $\mathrm{H}_{2} \mathrm{O}_{2}$ line list contained in HITRAN only covers wavelengths longer than $5 \mu \mathrm{m}$. PNNL [314] and recent $a b$ initio calculations [393,394] show strong features near $2.75 \mu \mathrm{m}$ associated with the $\mathrm{OH}$ stretch. More laboratory work is needed to obtain reliable line lists in that region.

\subsection{6. $\mathrm{C}_{2} \mathrm{H}_{2}$ (molecule 26)}

New bands of acetylene from the wavenumber regions $13-248 \mathrm{~cm}^{-1}$ and $390-634 \mathrm{~cm}^{-1}$ were added using the results of Refs. [395-397]. The new data incorporated into HITRAN2016 are 
based mainly on the global modeling of both line positions and intensities performed in Tomsk within the framework of the method of effective operators [395]. The new measurements performed by Jacquemart et al. [397] considerably extend the available experimental data in the $390-634 \mathrm{~cm}^{-1}$ region and allow an increase in the number of the fitted effective dipole moment (EDM) parameters responsible for the line intensities in the $13-634 \mathrm{~cm}^{-1}$ region. This extended set of EDM parameters has better extrapolation abilities compared to that published in Ref. [395]. Using the eigenfunctions of the effective Hamiltonian from Ref. [395] and the new set of EDM parameters, a new list of line positions and intensities was generated. This list was used to update the $\mathrm{C}_{2} \mathrm{H}_{2}$ line list in the $13-248 \mathrm{~cm}^{-1}$ and $390-634 \mathrm{~cm}^{-1}$ regions.

Global modeling of the rovibrational energies of the bending vibrational states has also been performed in the Jet Propulsion Laboratory (JPL) [396] using an EH for the bending modes. The comparisons to the observed line positions showed that the JPL calculated line positions are slightly closer to the observations for the high values of the angular momentum quantum numbers than those derived from Ref. [395]. Thus it was decided to use JPL line positions (when available) for these bands.

The line list has been completed by other parameters (selfand air-broadening coefficients, temperature exponents of airbroadening coefficients, air-shift coefficients) as described in Section 4 of Jacquemart et al. [398].

Uncertainty codes $4\left(0.0001\right.$ to $\left.0.001 \mathrm{~cm}^{-1}\right)$ for the line positions and $6(2-5 \%)$ for the line intensities were used in the case of the observed bands. For predicted bands, an uncertainty code 3 ( 0.001 to $\left.0.01 \mathrm{~cm}^{-1}\right)$ for the line positions and $4(10-20 \%)$ for the line intensities were adopted. For the JPL line positions, the uncertainty code $5\left(0.00001\right.$ to $\left.0.0001 \mathrm{~cm}^{-1}\right)$ was used.

The labeling $V_{1} V_{2} V_{3} V_{4} \ell_{4} \ell_{5} J \varepsilon$ of the ro-vibrational states has been used in the list; see Jacquemart et al. [397] for details.

In addition, it was discovered that the Q-branch in the $v_{2}+2 v_{4}+v_{5}$ band near $2.58 \mu \mathrm{m}$ was missing from the database. It is now provided based on the model of Lyulin and Perevalov [395].

In our effort to expand the database to spectral line parameters relevant to the study of planetary atmospheres, we have compiled semi-empirical parameter sets of $\mathrm{C}_{2} \mathrm{H}_{2}$ broadened and shifted by the pressure of $\mathrm{H}_{2}$, He and $\mathrm{CO}_{2}$ together with the corresponding temperature dependence of the linewidth. This update was described in detail in Wilzewski et al. [4]. These new parameters were constructed from Refs. [257,399-402] for $\mathrm{H}_{2}$, Refs. $[403,404]$ for He and Refs. $[405,406]$ for $\mathrm{CO}_{2}$.

Further studies of these line-shape parameters are important to improve our current update. This is especially true for the $\mathrm{C}_{2} \mathrm{H}_{2}-\mathrm{CO}_{2}$ system, where almost no shift parameters and no temperature-dependence values have been reported in the literature to this point.

We note a recent study by Lyulin and Campargue [407] where empirical line lists for some of the NIR bands of acetylene are provided. These will be considered for future updates of HITRAN.

\subsection{7. $\mathrm{C}_{2} \mathrm{H}_{6}$ (molecule 27)}

Ethane $\left(\mathrm{C}_{2} \mathrm{H}_{6}\right)$ is the second most abundant hydrocarbon after $\mathrm{CH}_{4}$ in the stratospheres of the outer planets (see for instance Ref. [408]) and Titan [409]. It therefore plays an important role as a tracer of atmospheric chemistry and dynamics. $\mathrm{C}_{2} \mathrm{H}_{6}$ is a direct product of $\mathrm{CH}_{4}$ photochemistry and is a key constituent in photochemical modeling. In addition to the $v_{9}$ fundamental of ethane, which is the strongest band seen in Titan observed in the $10-\mu \mathrm{m}$ terrestrial window, the weak $v_{4}$ torsional band at $\sim 35 \mu \mathrm{m}$ $\left(289 \mathrm{~cm}^{-1}\right)$ has been detected on Titan by Cassini/CIRS [410]. Although the torsional band is not infrared active in the lowest order and hence very weak in a laboratory setting, the features near 35 $\mu \mathrm{m}$ are seen relatively strong in the Cassini/CIRS spectrum of Titan. This new development in the far-infrared enables astronomers to probe the lower altitudes of Titan's atmosphere.

\subsection{1. ${ }^{12} \mathrm{C}_{2} \mathrm{H}_{6}$}

Since the last update in HITRAN2012 [1] for ${ }^{12} \mathrm{C}_{2} \mathrm{H}_{6}$ at $12 \mu \mathrm{m}$ reported by Devi et al. $[411,412]$ and at $7 \mu \mathrm{m}$ reported by di Lauro et al. [413], significant progress has been made for ethane in the far- and mid-infrared regions. Moazzen-Ahmadi et al. [414] studied the features in the $35-\mu \mathrm{m}$ region both in laboratory measurements and theoretical modeling, which included the torsional fundamental $v_{4}$, and the first torsional hot band, $2 v_{4}-v_{4}$, of ${ }^{12} \mathrm{C}_{2} \mathrm{H}_{6}$ and the Q-branch of the ${ }^{13} \mathrm{CH}_{3}{ }^{12} \mathrm{CH}_{3} v_{6}$ band. Multiple sets of pure ethane spectra were obtained at cold temperatures down to $166 \mathrm{~K}$ at the Jet Propulsion Laboratory using a multipass absorption cell with 52-m path length. Starting with the earlier global analyses of ethane considering inter-vibrational interactions among the torsional levels of $v_{3}, v_{9}, 3 v_{4}$ bands [415], the observed spectra were fit by adjusting independent dipole constants and the same value for self-broadening parameter for every transition. The observed spectra were reproduced to the residuals of $1 \%$ (the reader is referred to Fig. 1 in Ref. [415]).

It was found that the modeling of the transition intensities required an expansion of the dipole moment operator to higher order; this introduced Herman-Wallis like terms. The line positions and line intensities of the torsional band $\left(v_{4}\right)$ of ${ }^{12} \mathrm{C}_{2} \mathrm{H}_{6}$ at $35 \mu \mathrm{m}$ reported by Moazzen-Ahmadi et al. [414] have been adopted in this update. The uncertainties for line positions are set to no better than $0.0003 \mathrm{~cm}^{-1}$ (HITRAN code $=4$ ), while those of the line intensities are conservatively set to be 5 to $10 \%$ (HITRAN code $=5$ ). For the pressure-broadened widths of the torsional band $\left(v_{4}\right)$ at $35 \mu \mathrm{m}$, it should be noted that self- and $\mathrm{N}_{2}$-broadened widths are listed in this version, for which we adopted the values calculated by using the linear expressions for $v_{9}$ transitions reported by Devi et al. $[411,412]$, similar to what was done for $\mathrm{C}_{2} \mathrm{H}_{6}$ in the $7-\mu \mathrm{m}$ region in HITRAN2008 [198]. The uncertainties for widths are conservatively set to $10-20 \%$ (HITRAN code $=4$ ). The uncertainties for the temperature dependence exponents of the widths are thought to be $10-25 \%$ (HITRAN code $=3$ ).

In the near future two minor isotopologues of ethane could be updated in the $12 \mu \mathrm{m}$ region; one is for the ${ }^{13} \mathrm{C}$ singly-enriched $v_{12}$ band of ethane $\left({ }^{13} \mathrm{CH}_{3}{ }^{12} \mathrm{CH}_{3}\right)$ at $12.2 \mu \mathrm{m}$ measured by Devi et al. [416] and the other for the singly-deuterated ethane $\left(\mathrm{C}_{2} \mathrm{H}_{5} \mathrm{D}\right)$ at $12.5 \mu \mathrm{m}$ studied by Daly et al. [417]. In particular, new studies were reported for ${ }^{13} \mathrm{CH}_{3}{ }^{12} \mathrm{CH}_{3}$ in the $v_{12}$ band in the $12 \mu \mathrm{m}$ region by Devi et al. [416] and in the $7 \mu \mathrm{m}$ region by di Lauro et al. [418] for the $v_{4}, v_{9}, v_{10}$ and $v_{6}+v_{11}$ bands between 1345 and $1557 \mathrm{~cm}^{-1}$. Their intensities were reported at $130 \mathrm{~K}$ with partially completed theoretical modeling.

\subsection{8. $\mathrm{PH}_{3}$ (molecule 28)}

Phosphine $\left(\mathrm{PH}_{3}\right)$ is a molecule of interest in astronomy, and its features have long been observed and identified in the spectra of Jupiter and Saturn (e.g. Refs. [419-422]). Because of its great importance in the Earth's and other planetary atmospheres including the interstellar medium, $\mathrm{PH}_{3}$ has been the subject of a large number of experimental and theoretical investigations. Far infrared (FIR) line parameters of $\mathrm{PH}_{3}$ were included for the first time in the HITRAN2012 database [1]. No updates have been made for any of the spectral line parameters for the infrared (IR) bands of phosphine since the HITRAN2008 database [198]. After the release of the HITRAN2012 database, there have been a few experimental and theoretical studies reported on phosphine [423-426]. Sousa-Silva et al. $[423,424]$ generated a line list for phosphine; their room temperature line list consists of 137 million transitions among the 5.6 
million energy levels for the ro-vibrational states up to $J_{\max }=31$ and energies up to $8000 \mathrm{~cm}^{-1}$. It was augmented by a more extensive and more accurate "hot" line list capable of simulating observed spectra from astronomical bodies at higher temperatures (e.g. $1500 \mathrm{~K}$ ). In the near infrared region that is important for remote sensing of the outer planets, especially Jupiter and Saturn, the two most extensive and comprehensive experimental measurements of phosphine to date include: (1) the line positions and intensities for the five bands $\left(2 v_{2}, v_{2}+v_{4}, 2 v_{4}, v_{1}\right.$ and the $\left.v_{3}\right)$ in the pentad region between 1950 and $2450 \mathrm{~cm}^{-1}$ [426] and (2) the measurements of spectral line-shape parameters (e.g., pressurebroadened half widths, pressure shifts, and collisional line mixing coefficients) for the $v_{2}+v_{4}, 2 v_{4}, v_{1}$ and the $v_{3}$ bands [425].

The measurements reported in Ref. [426] show an increase in the number of individual fitted intensities for the fundamental bands $v_{1}$ and $v_{3}$ from 20 and 50 reported in the previous laboratory measurements of Tarrago et al. [2.2.28.11] to 184 and 409, respectively. The actual number of measured intensities in these bands were 337 and 829 , respectively; however only the best selected lines were used in the determination of the pentad band strength. Positions and intensities for more than 4000 individual transitions in the pentad were retrieved from analyses of the FTS spectra recorded at the National Solar Observatory (NSO) on Kitt Peak, and the PNNL spectra [314]. The results published in Refs. $[425,426]$ included $53 \mathrm{~A}+\mathrm{A}$ - doublet components that are split by the Coriolis interaction between the $v_{1}$ and $v_{3}$ bands, and nearly 150 vibrationally-mixed perturbation-allowed transitions. The predicted spectrum from the analysis, however, did not reproduce the experimental spectrum fully because the precisions of the measurements were better than the calculations (for example see Fig. 9 from Ref. [426]). To improve the calculations, a global vibrational and intensity analysis combining the pentad measurements $[425,426]$ with data in the dyad and the octad regions should be undertaken.

It appears that the SAITY line list from the recent $a b$ initio calculations [424] is in much better agreement with Ref. [426] than with the HITRAN2012 data. Figure 22 shows a comparison of the SAITY line list [424] with the phosphine data from HITRAN2012 [1] at room temperature; also shown are the recent results of Malathy Devi et al. [426].

Recently, Nikitin et al. [427] have reported theoretical predictions of phosphine spectra based on $a b$ initio dipole moment [428] and potential energy surfaces [429]. These calculations permitted a qualitatively correct agreement with observed data up to the octad range $\left(2733-3660 \mathrm{~cm}^{-1}\right)$. An example of the comparison between these $a b$ initio and experimental PNNL spectra [314] is given in Fig. 23. The theoretical approach to these variational calculations is described in Rey et al. [430] and the corresponding line list and $a b$ initio cross-sections in the range $\left(0-7000 \mathrm{~cm}^{-1}\right)$ is available in the TheoReTS database [205].

The $a b$ initio calculations described above (especially line positions) may not be of sufficient quality to model room-temperature high resolution spectra to the HITRAN standard of accuracy. However, they are very helpful in assigning experimental spectra and invaluable in high-temperature simulations and will be considered for inclusion in a future edition of the HITEMP database [278].

The theoretical background and the set of programs used for both the energy and intensity fittings in the analysis of Malathy Devi et al. are described in Ref. [426]. Along with the positions and intensities, line-shape parameters were measured for a large number of transitions (over 840 Lorentz self-broadened half width coefficients, 620 self-shift coefficients, 185 speed dependence parameters, and collisional line-mixing parameters for $10 \mathrm{~A}+\mathrm{A}$ - doublet transitions) in the $2 v_{4}, v_{1}$ and $v_{3}$ bands, and those measurements are reported in a separate article [425]. As mentioned in the analysis of positions and intensities [426], the parameters in

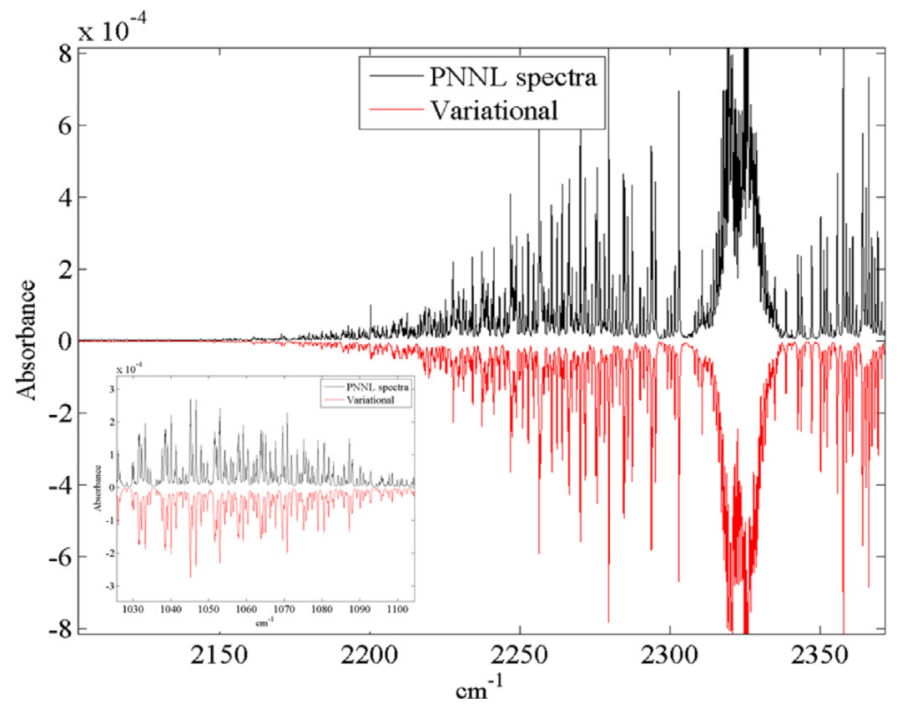

Fig. 23. Comparison of experimental PNNL absorbance cross-sections [314] of $\mathrm{PH}_{3}$ in the dyad and pentad ranges (in black) with variational calculations Rey et al. [205] (upside down in red).

the pentad region are important for the exploration of chemistry and dynamics on Saturn using the existing Cassini/VIMS observations. The results reported in Refs. [425,426] (which have appropriate supplementary materials) are applicable to a variety of remotesensing studies including the analyses of the near infrared data of Jupiter from the Juno mission and ESA's Jupiter Icy Moons Explorer (JUICE).

Creation of a line list of $\mathrm{PH}_{3}$ in the pentad and octad regions is in progress and is expected to be available as an update to the HITRAN2016 database.

\subsection{9. $\mathrm{COF}_{2}$ (molecule 29)}

Unchanged.

\subsection{0. $\mathrm{SF}_{6}$ (molecule 30$)$}

Unchanged.

\subsection{1. $\mathrm{H}_{2} \mathrm{~S}$ (molecule 31)}

Line positions for 654 transitions in the (010)-(000) band were updated using experimental upper- state energy levels for $\mathrm{H}_{2}{ }^{32} \mathrm{~S}$, $\mathrm{H}_{2}{ }^{33} \mathrm{~S}$, and $\mathrm{H}_{2}{ }^{34} \mathrm{~S}$ isotopologues reported in Ulenikov et al. [431]. while the lower-state energy levels were calculated using the rotational constants of Flaud et al. [432]. It appeared that the band center of this band was off by about $0.002 \mathrm{~cm}^{-1}$ in HITRAN2012 while some higher- $J$ rotational transitions were off by up to $\sim 0.055 \mathrm{~cm}^{-1}$.

In the triad region it was found that values for different parameters for some of the lines were not transferred correctly from Ref. [433] to HITRAN2000 [434]. This issue remained all the way through HITRAN2012, but has been fixed for HITRAN2016.

Intensity distribution of some of the multiplets in the NIR region was found to be inaccurate in the previous version in HITRAN for many lines where the experimental intensity for the entire multiplet were given to every component in the multiplet, rather than the intensity being divided between components. This is fixed in HITRAN2016.

The air-broadening coefficients of hydrogen sulfide $\left(\mathrm{H}_{2} \mathrm{~S}\right)$ in the HITRAN database were set to a constant value, $\gamma_{\text {air }}=0.0740 \mathrm{~cm}^{-1}$ $\mathrm{atm}^{-1}$ at $296 \mathrm{~K}$, for most of the transitions (since HITRAN2004 [3]) 

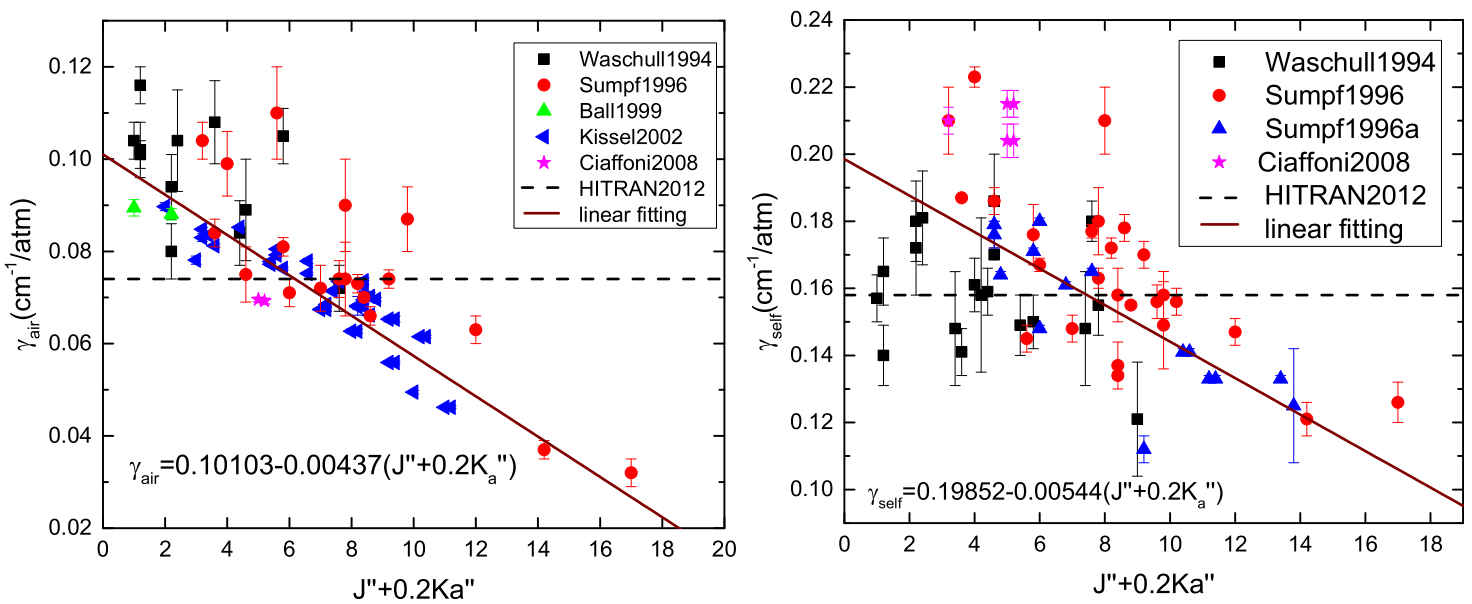

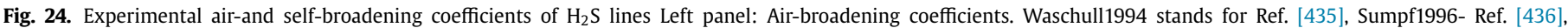
Ball1999- Ref. [440], Kissel2002-Ref. [437], Ciaffoni2008- Ref. [441]. Right panel: Self-broadening coefficients. Sumpf1996s stands for Ref. [438].

based on the average value of available experimental data while just a few half widths were based on experimental values. For HITRAN2016, a thorough survey of measurements of both the airbroadening and self-broadening coefficients of $\mathrm{H}_{2} \mathrm{~S}$ has been made [435-438]. The report by Tan et al. [439] describes the results in more detail. In brief, a slow decrease of the air-(and self-) broadening coefficients with increasing $\left(J^{\prime \prime}+0.2 K_{a}^{\prime \prime}\right)$ lower-state quantum number combination can be seen in Fig. 24. The vibrational dependence of the broadening parameters is neglected and we use the semi-empirical method based on the linear fit to the available experimental data to compute both air- and self-broadening coefficients of $\mathrm{H}_{2} \mathrm{~S}$ for all the isotopologues. This linear approaching value could only be used within the range probed by experiments. So for lower-state rotational quantum number $J^{\prime \prime}<12$, the error code was set to 4 (between 10 and 20\%) for both self- and airbroadening coefficients; for $12 \leq J^{\prime \prime} \leq 18$, the error code was set to 3 ( $>20 \%$ ); and for $J^{\prime \prime}>18$, the value of both self- and air-broadening coefficients was set to the linear fit value corresponding to $J^{\prime \prime}=18$, and the error code was 2 (estimate).

\subsection{2. $\mathrm{HCOOH}$ (molecule 32)}

Unchanged.

\subsection{3. $\mathrm{HO}_{2}$ (molecule 33)}

The air-broadening coefficients of the hydroperoxyl radical had not been modified since HITRAN2004 [3] in which they were set to a constant, $\gamma_{\text {air }}=0.107 \mathrm{~cm}^{-1} / \mathrm{atm}$ (based on the measurement in Ref. [442]). In the meantime, a number of different measurements of $\gamma_{\text {air }}$ have been performed using infrared and submillimeterwave spectra [442-447]. These measurements indicate a small vibrational dependence and a slow linear dependence with rotational quanta for $\gamma_{\text {air }}$.

The following approach was chosen for the air-broadening values in HITRAN2016. Experimental values were used wherever available for all the transitions with the corresponding rotational quantum numbers (in all the bands). For all other lines, a linear extrapolation with $\left(J^{\prime \prime}+0.2 K_{a}^{\prime \prime}\right)<17$ was used:

$\gamma_{\text {air }}=0.1367-0.0027 \times\left(J^{\prime \prime}+0.2 K^{\prime \prime} a\right)$

For all transitions with $\left(J^{\prime \prime}+0.2 K_{a}{ }^{\prime \prime}\right)>17$, the value corresponding to the experimental value with the largest $\left(J^{\prime \prime}+0.2 K_{a}^{\prime \prime}\right)$ was used. More details on the aforementioned algorithm are given in the report by Tan et al. [448].
For the self-broadening, a default estimate value of $0.3 \mathrm{~cm}^{-1} / \mathrm{atm}$ was assigned to all transitions.

\subsection{O ("molecule" 34)}

The values of line positions and lower-state energies for the 0 atom have been reverted to those given in the HITRAN1996-2000 editions $[434,449]$. They originate from Zink et al. [450]. The HITRAN2004 to 2012 editions used outdated values from the older version of the JPL catalogue [451] (downloaded in 2004), although the reference code mistakenly pointed to the work of Zink et al. The values have since been fixed in the JPL catalogue [451] (downloaded in 2015), and consequently we reverted back to the more accurate values of Zink et al. [450].

\subsection{5. $\mathrm{ClONO}_{2}$ (molecule 35)}

Unchanged.

\subsection{6. $\mathrm{NO}^{+}$(molecule 36)}

The fundamental band of the $\mathrm{NO}^{+}$molecule (in groundelectronic state ${ }^{1} \Sigma^{+}$) as well as its hot bands were introduced into the HITRAN1996 edition [449] and updated in 2008 [198]. As pointed out by López-Puertas et al. [452] and references therein, the molecule is an important constituent of Earth's ionosphere. Ref. [452] actually used atmospheric observations to determine transition frequencies of its fundamental band and the associated hot band. The identification of $\mathrm{NO}^{+}$in the terrestrial atmosphere by rotational spectroscopy should be possible. In addition, $\mathrm{NO}^{+}$was recently identified tentatively in the interstellar medium [453].

The new edition of HITRAN adds the previously omitted purerotation transitions. The current prediction of the rotational spectrum corresponds to the recently created version 2 of the CDMS [454]. The data are based on a fit to rotational data [455] as well as ro-vibrational data $[452,456]$. The dipole moment of $0.380 \mathrm{D}$ is from a quantum-chemical calculation [457].

The ground state rotational data extend to the $J=8-7$ transition near $32 \mathrm{~cm}^{-1}$. Therefore, predictions should be reliable to at least up to $70 \mathrm{~cm}^{-1}$. The entry now also contains predictions of rotational transitions in $\mathrm{v}=1$; these are more uncertain than those in $\mathrm{v}=0$.

\subsection{7. $\mathrm{HOBr}$ (molecule 37)}

Unchanged. 


\subsection{8. $\mathrm{C}_{2} \mathrm{H}_{4}$ (molecule 38)}

Ethylene, $\mathrm{C}_{2} \mathrm{H}_{4}$, is both a naturally-occurring compound and a tropospheric pollutant on the Earth [458]. It is also present as a by-product of methane photochemistry in the atmosphere of outer solar system bodies including Saturn [459] and Titan [409]. Optical remote sensing of ethylene in planetary atmospheres often relies on the strongest band observed in the $10-\mu \mathrm{m}$ spectral region, namely the $v_{7}$ fundamental of the main isotopologue [460]. The extensive work carried out in this range of the ro-vibration spectrum of ${ }^{12} \mathrm{C}_{2} \mathrm{H}_{4}$ was recently reviewed by Alkadrou et al. [461]. The data available in HITRAN2012 for the $10-\mu \mathrm{m}$ region corresponds to the infrared active $v_{10}, v_{7}$ and $v_{12}$ bands of ${ }^{12} \mathrm{C}_{2} \mathrm{H}_{4}$, and the infrared and Raman inactive $v_{4}$ band of the same isotopologue, located near 826, 949, 1442 and $1026 \mathrm{~cm}^{-1}$, respectively. The information for the $v_{10}, v_{7}$ and $v_{4}$ bands dates back to the 2000 edition of HITRAN [434]. It was generated relying on the frequency analysis of Willaert et al. [462], Cauuet et al. [463], Legrand et al. [464] and Rusinek et al. [465], and intensities measured for 13 lines of the $v_{7}$ band observed in the range 940-970 $\mathrm{cm}^{-1}$ [466]. Spectroscopic information obtained for the $v_{12}$ band by Rotger et al. [467] was added in the 2008 edition [198].

A global frequency and intensity analysis of the $10-\mu \mathrm{m}$ $v_{10} / v_{7} / v_{4} / v_{12}$ band system of ${ }^{12} \mathrm{C}_{2} \mathrm{H}_{4}$ was very recently carried out using the tensorial formalism developed in Dijon for $\mathrm{X}_{2} \mathrm{Y}_{4}$ asymmetric-top molecules and including the Coriolis interactions affecting the upper vibrational levels $10^{1}, 7^{1}, 4^{1}$ and $12^{1}$ [461]. Relying on spectroscopic information available in the literature or specifically retrieved from high-resolution Fourier transform infrared spectra, a total of 10,757 line positions and 1645 line intensities were assigned and fitted with global root mean square deviations of $2.6 \times 10^{-4} \mathrm{~cm}^{-1}$ and $2.5 \%$, respectively. Using the results of that work and information available in the literature, a HITRANformatted list of parameters for 65,776 lines belonging to the $v_{10}$, $v_{7}, v_{4}$ and $v_{12}$ bands of ${ }^{12} \mathrm{C}_{2} \mathrm{H}_{4}$ and covering the $620-1525 \mathrm{~cm}^{-1}$ range was generated [461]. Comparisons of experimental spectra with spectra calculated for the same physical conditions with this line list and HITRAN 2012 are presented in Figs. 5 to 7 of Ref. [461]. They show that the modeling provided by this line list is significantly better than HITRAN 2012 for the $v_{10}$ band, comparable for the $v_{7}$ band (and for the $v_{4}$ band hidden beneath it), and slightly worse for the $v_{12}$ band.

In the line list of Ref. [461], the vibration-rotation levels are assigned using the traditional $\left(J, K_{a}, K_{c}\right)$ labels, where $J$ is the quantum number associated with the total angular momentum of the molecule, and $K_{a}$ and $K_{c}$ are associated with its projections along the molecular symmetry axes corresponding to the limiting prolate and oblate symmetric top approximations. As the tensorial formalism characterizes these levels using ( $, \mathrm{C}, \alpha$ ) labels ( $\mathrm{C}$ is the rovibrational symmetry in the $\mathrm{D}_{2 \mathrm{~h}}$ group, and $\alpha$ is a ranking number used to sort the energy levels within a (J, C) block), a "translation" had to be carried out when generating the line list. It was very recently found that $K_{a}$ and $K_{c}$ labels were incorrect for a number of heavily-mixed rotational levels of the $10^{1}, 7^{1}$ and $4^{1}$ upper vibrational levels. A corrected line list was therefore generated and submitted for publication [468].

The spectroscopic information available in HITRAN for the 10$\mu \mathrm{m}$ region of ${ }^{12} \mathrm{C}_{2} \mathrm{H}_{4}$ has been updated in the following way. Data for the $v_{10}, v_{7}$ and $v_{4}$ bands were taken from the corrected line list of Alkadrou et al. [468]; however, lines having intensity smaller than $10^{-30} \mathrm{~cm}^{-1} /\left(\right.$ molecule $\mathrm{cm}^{-2}$ ) at $296 \mathrm{~K}$ were ignored. The line positions are given a HITRAN error code of 4 or $0(0.0001-$ $0.001 \mathrm{~cm}^{-1}$ and larger than $1 \mathrm{~cm}^{-1}$ respectively) depending on whether the corresponding $J^{\prime \prime}$ and $K_{a}^{\prime \prime}$ values are or are not in the ranges included in the frequency analysis of Ref. [461]. Similarly, an error code of 5 (5-10\%) characterizes the intensities of lines in the $v_{10}$ and $v_{7}$ bands corresponding to transitions involving $J^{\prime \prime}$ and $K_{a}^{\prime \prime}$ values in the ranges included in the intensity analysis of Ref. [461]. The error code is set to 0 for all other line intensities. Note that errors in the degeneracies provided for the rotational levels in the $10^{1}$ and $7^{1}$ vibrational levels in the line lists of Refs. [461] and [468] are corrected here. Data for the $v_{12}$ band was taken from HITRAN2012; however, 187 lines having an intensity smaller than $10^{-30} \mathrm{~cm}^{-1} /\left(\right.$ molecule $\left.\mathrm{cm}^{-2}\right)$ at $296 \mathrm{~K}$ have been excluded, to be consistent with the information provided for the three other bands. For all the lines, the self-broadening coefficients were calculated using the empirical expressions reported in Ref. [460], the air-broadening coefficients were generated as described in [467], and the temperature dependence exponent $n$ was set to 0.76 , determined as described in Ref. [461].

The consistency of the $\mathrm{C}_{2} \mathrm{H}_{4}$ analyses that served for the updated line list has been confirmed by extensive variational calculations [469] based on ab initio potential energy [470] and dipole moment surfaces [471]. This sort of qualitative band-shape validation was found to be particularly important for modeling complicated intensity perturbations involving "dark" states and for checking for possible contributions of hot bands. The corresponding variational methods are described by Rey et al. [469], and the results of $a b$ initio based calculations including cold and hot bands in the range $0-6000 \mathrm{~cm}^{-1}$ can be found in the TheoReTS database [205]. These calculations agree well with the new HITRAN2016 line list and experimental PNNL spectra [314] in the 10- $\mu \mathrm{m}$ region.

New bands of ${ }^{12} \mathrm{C}^{13} \mathrm{CH}_{4}$ are included in the new version of the database. More precisely, the new parameters which were derived from high-resolution Fourier transform spectra are those of the $v_{10}, v_{8}, v_{7}, v_{4}, v_{6}$ bands, which absorb in the $615-1339 \mathrm{~cm}^{-1}$ spectral region $[472,473]$. Their accuracy can be estimated to be of the order of $0.6 \times 10^{-3} \mathrm{~cm}^{-1}$ for the positions [473] and $4 \%$ for the intensities [472]. The broadening parameters were determined in the same fashion as for the principal isotopologue described above.

Fig. 25 (generated using HAPI described in Section 6) shows that the HITRAN2016 ethylene line list models experimental spectra from PNNL [314] much better than HITRAN2012.

\subsection{9. $\mathrm{CH}_{3} \mathrm{OH}$ (molecule 39)}

It was found that lower-state energies in the IR spectra of methanol introduced in HITRAN2004 [3] do not follow HITRAN formalism in that the lowest allowed energy level should be equal to zero. Yet the convention was observed in the MW region. The lowest energy in the IR spectra was $128.1069 \mathrm{~cm}^{-1}$ instead of zero. This has been fixed now to follow the usual convention.

\subsection{0. $\mathrm{CH}_{3} \mathrm{Br}$ (molecule 40)}

\section{Unchanged.}

\subsection{1. $\mathrm{CH}_{3} \mathrm{CN}$ (molecule 41)}

Methyl cyanide, also known as acetonitrile or cyanomethane, a trace species in the terrestrial atmosphere [474], has also been seen in comets [475] and in the atmosphere of Titan [476], and is an important species in various environments of the interstellar medium. Müller et al. [479] provided a brief overview as well as appropriate references. The $v_{4}$ band of methyl cyanide was introduced into HITRAN2008 [198]. The data were based on a multispectrum analysis of this band and a preliminary modeling of the positions and intensities [477,478]. The $v_{4}$ band is actually the third lowest wavelength band, with $v_{8}$ and $2 v_{8}$ being at longer wavelengths. The prospects of detecting transitions of $v_{8}$ or $2 v_{8}$ with the James-Webb Space Telescope or other instruments in space or in Earth's atmosphere are uncertain. The data are thought 

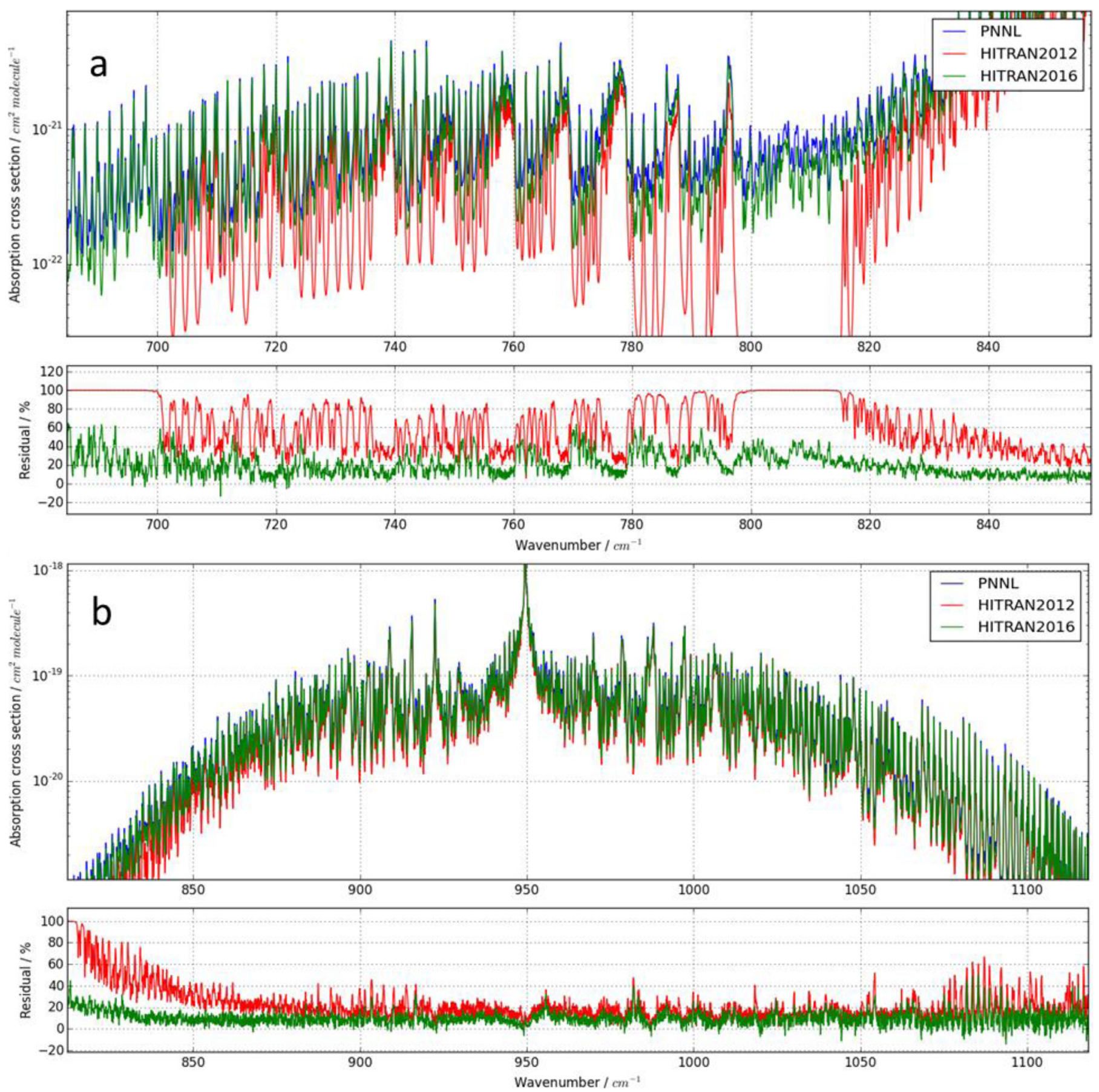

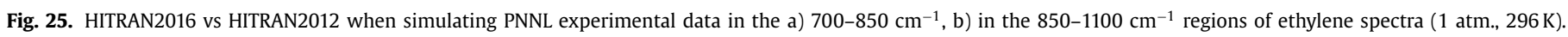

to be important for the excitation or de-excitation of the $\mathrm{v}=0$ transitions or of bands with much greater intensity, such as $v_{6}$.

The following update is planned in the immediate future, following the HITRAN2016 release. The current line position and intensity predictions for $v_{8}, 2 v_{8}$, and $2 v_{8}-v_{8}$ are from the CDMS [454]; the entries are actually combined CDMS and JPL entries. The predictions are based on a combined fit of rotational and rovibrational data involving states up to $\mathrm{v}_{8}=2$ that was published by Müller et al. [479]. The analysis takes into account interactions among these states as well as interactions of $\mathrm{v}_{8}=2$ with $\mathrm{v}_{4}=1, \mathrm{v}_{7}$ $=1$, and $v_{8}=3$ and of the last three states among each other.
The $v_{8}$ transition frequencies were taken from Koivusaari [480], and the $2 v_{8}$ transition frequencies were from Müller et al. [479]. Most of the $\mathrm{v}_{8}=1$ and 2 rotational data as well as some $\mathrm{v}=0$ data are also from Müller et al. [479]. Most of the additional groundstate data were published by Cazzoli and Puzzarini [481] and by Müller et al. [482]. Information of the $K$ level structure in the ground vibrational state is based on Anttila et al. [483] and on the perturbations treated in Ref. [479]. Additional $\mathrm{v}_{8}=1$ and 2 data were published by Bauer et al. [484]. Parameters describing the three states around $10 \mu \mathrm{m}$ were taken mostly from the study of Tolonen at al. [485]. 


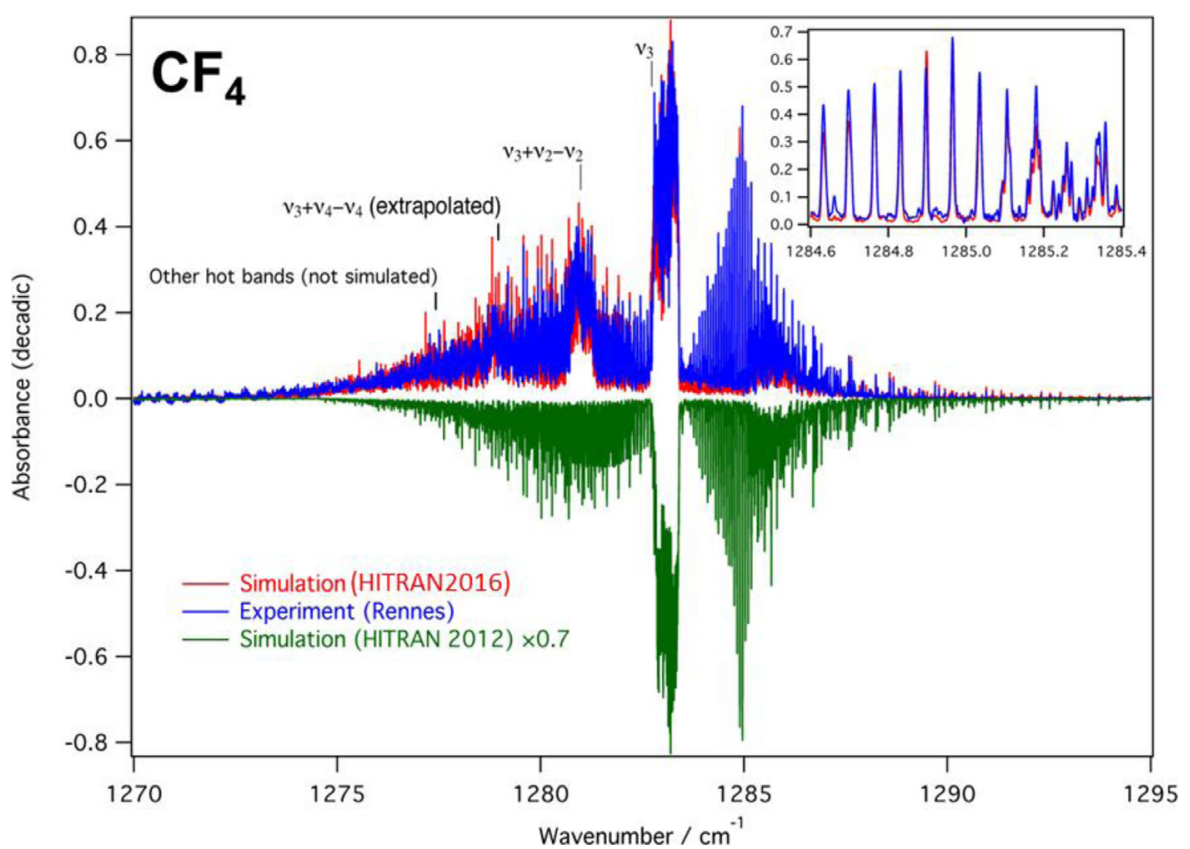

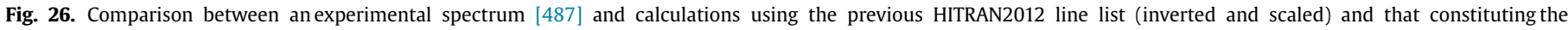
HITRAN2016 update.

The transition dipole moments were evaluated by Müller at al. [479] as were line broadening and shifting parameters which were transferred from the $v_{4}$ band [477]. The predictions should be reliable throughout, but slight caution is recommended for the highest $K$ transitions of $2 v_{8}(K=13$ and 14$)$.

Assignments for the $v_{4}$ band are probably complete as far as the available spectral recordings are concerned. However, the preliminary analysis in Ref. [478], together with data from Ref. [479], affected the ground-state parameters substantially such that a new modeling effort will be necessary. The analysis should be completed well before the next HITRAN update, and further data may be available as well.

\subsection{2. $\mathrm{CF}_{4}$ (molecule 42)}

Carbon tetrafluoride, $\mathrm{CF}_{4}$, is a greenhouse gas with a huge global warming potential. Up to now, only the $v_{3}$ fundamental band was present in HITRAN. However, this band represents only about $65 \%$ of the absorption intensity at room temperature due to the presence of hot bands.

A new global fit of all $\mathrm{CF}_{4}$ assigned lines involving the $v_{2}$, $v_{3}$ and $v_{4}$ modes, as well as a separate one consisting of the $v_{1}$ mode have been performed, including some new experimental data which was recently recorded in the Groupe de Spectrométrie Moléculaire et Atmosphérique, Université de Reims-ChampagneArdenne. This study [486] will be published soon. It has resulted in new reliable effective Hamiltonian parameters for ${ }^{12} \mathrm{CF}_{4}$, which, in turn, allow an improved calculation of the $v_{4}$ and $v_{3}$ fundamental bands and, also and for the first time, of the $v_{3}+v_{2}-v_{2}$ hot band which represents about $17 \%$ of the absorption intensity in this region at room temperature. The dipole moment parameters have also been corrected. This will be explained in detail in Ref. [486]. Thus, the present update completely replaces the previous data and includes the $v_{4}, v_{3}$ and $v_{3}+v_{2}-v_{2}$ bands.

Fig. 26 shows a comparison between an experimental spectrum from the Rennes group [487] and the calculation constituting the HITRAN2016 update.

\subsection{3. $\mathrm{C}_{4} \mathrm{H}_{2}$ (molecule 43)}

The $v_{9}$ band region (around $220 \mathrm{~cm}^{-1}$ ) and the $v_{8}$ band region (around $650 \mathrm{~cm}^{-1}$ ) of diacetylene, $\mathrm{C}_{4} \mathrm{H}_{2}$, is important in interpreting Titan [488] observations. The $v_{9}$ band region has been completely replaced with a new line list derived from a combined analysis presented in Refs. [488,489]. The updated line list provides new intensity for the lines already existing in the database but also includes numerous hot bands. The $v_{8}$ band region was updated by scaling the intensities of all the lines in that region by a factor of 0.8 based on the recommendation given in Jolly et al. [489]. Finally, the $v_{6}+v_{8}$ region (around $1200 \mathrm{~cm}^{-1}$ ) was introduced into HITRAN for the first time based on the predictions from the global analyses. This band is important in astrophysics as it has been observed in the atmosphere of Titan and the preplanetary nebula SMP LMC 11 with the Spitzer Infrared Spectrograph [490].

\subsection{4. $\mathrm{HC}_{3} \mathrm{~N}$ (molecule 44)}

Unchanged.

\subsection{5. $\mathrm{H}_{2}$ (molecule 45)}

Molecular hydrogen $\left(\mathrm{H}_{2}\right)$ is the most abundant gas in the atmosphere of gaseous giants. It was first introduced in HITRAN2012 [1]. The line list covered the quadrupole-allowed transitions for the $\mathrm{H}_{2}$ isotopologue and dipole-allowed transitions for HD in their ground electronic states.

An update was made for singly-deuterated molecular hydrogen (HD) shortly after the release of the HITRAN2012 database. It was discovered by J. Mendrok (Lulea University of Technology, Kiruna, Sweden) that the abundance of the HD molecule was incorrectly calculated in the original release of HITRAN2012; this issue has now been fixed. The implication was that the intensities of the electric dipole transitions originally reported needed to be multiplied by a factor of two, although it did not affect the Einstein-A coefficients. 
In addition, a total of 7195 electric quadrupole transitions of the HD molecule have been calculated and added to the HITRAN line list. The calculation was carried out using the energy levels from the work by Pachucki and Komasa [491] and the quadrupole moment function of Wolniewicz et al. [492].

In HITRAN2016, 527 transitions of $\mathrm{H}_{2}$ that correspond to high vibrational overtones were removed. This was because there were numerical issues in calculating the intensities of these transitions. Refs. [340,341] explain how the use of double precision in calculating overtone intensities may lead to numerical errors and that quadruple precision is needed.

Only a default value of the broadening parameter for the Voigt profile was given (the same for all the lines) in HITRAN2012. In HITRAN2016, a complete dataset of the line-shape parameters for the Hartmann-Tran profile is provided for self-perturbed $\mathrm{H}_{2}$ [6]. This dataset was determined from the analysis of $\mathrm{H}_{2}$ experimental spectra. According to the new recommendation adopted in HITRAN, these line-shape parameters are stored separately for four different temperature ranges, see Ref. [6]. For the lines and conditions for which experimental spectra are not available, the lineshape parameters were extrapolated. In the near future we plan to extend HITRAN with the HT line-shape parameters for $\mathrm{H}_{2}$ perturbed by helium, which will be based on experimentally validated ab initio calculations [493].

\subsection{CS (molecule 46)}

\section{Unchanged.}

\subsection{7. $\mathrm{SO}_{3}$ (molecule 47)}

Sulfur trioxide $\left(\mathrm{SO}_{3}\right)$ occurs naturally in volcanic emissions and hot springs [494]. It is also a pollutant emitted by smoke stacks and other industrial exhausts [495]. In the terrestrial atmosphere, $\mathrm{SO}_{3}$ rapidly forms sulfuric acid with its association with acid rain. $\mathrm{SO}_{3}$ is a constituent in the atmosphere of Venus [496]. Recently, Zhang et al. [497] have indicated that measurements of the abundances of $\mathrm{SO}_{3}$ in the lower thermosphere of Venus can answer important questions related to sulfur chemistry. The spectroscopic study of sulfur oxides can also provide insight into the history of the Earth's atmosphere [498] and hence modeling of Earth-like planets.

$\mathrm{SO}_{3}$ was included for the first time in the 2012 HITRAN edition. This entry was based on the experimental data of Maki and co-workers [499-502] with relative transition intensities scaled by the absolute values computed $a b$ initio by Underwood et al. [503]. Also included were 25 pure-rotation transitions observed by Meyer et al. [504] for which the $a b$ initio intensities of Underwood et al. [503] were used.

$\mathrm{SO}_{3}$ is a planar, non-polar molecule but with sufficient rotational excitation that the molecule can distort and undergo pure-rotational transitions. A hybrid, empirical ab initio rotational line list for ${ }^{32} \mathrm{~S}^{16} \mathrm{O}_{3}$ has been constructed for this edition of the database. For this, the ground-state rotational constants and centrifugal distortion parameters were taken from the fits to their infrared spectrum given by Ref. [502]; these were used to generate a set of vibrational ground-state rotational energies for $J$ up to 85 . To ensure that the extrapolation to high- $J$ states is reasonable, a comparison was made between these energy levels and those calculated variationally by Ref. [505]; very good agreement was found with a root-mean-square difference of only $0.0167 \mathrm{~cm}^{-1}$ for all rotational transitions involving states with $J$ up to 85 . Intensities (and Einstein-A coefficients) were determined using the variational calculations and an $a b$ initio dipole moment surface [503]. The result is a list of 3414 pure-rotational transitions for ${ }^{32} \mathrm{~S}^{16} \mathrm{O}_{3}$ which have been added to the 25 microwave transitions previously included.
No measurements or calculations for the line-shape parameters are available; therefore the usual default values were chosen.

Recently Underwood et al. [506] presented a combined theoretical study of the spectrum of hot ${ }^{32} \mathrm{~S}^{16} \mathrm{O}_{3}$. This paper provided an extensive new line list with 21 billion lines for hot ${ }^{32} \mathrm{~S}^{16} \mathrm{O}_{3}$ but no new line measurements. However, comparisons between this line list and the infrared absorption measurements suggest that the calculations overestimate the infrared line intensities by about $30 \%$. Interestingly, this difference appears to be rather uniform. Some direct measurement of the ${ }^{32} \mathrm{~S}^{16} \mathrm{O}_{3}$ infrared line intensities would be welcome to inform future updates of the database.

\subsection{8. $\mathrm{C}_{2} \mathrm{~N}_{2}$ (molecule 48)}

Cyanogen is present in the atmosphere of Titan [507]. Its line list is now included into HITRAN for the first time based on the work of Fayt et al. [508]. The line list is for the $v_{5}$ band system around $234 \mathrm{~cm}^{-1}$.

\subsection{9. $\mathrm{COCl}_{2}$ (molecule 49)}

Phosgene is a long-lived, highly toxic synthetic gas that is widely used in pharmaceutical and polymer industries. Phosgene is a serious health hazard and was, in fact, used as a chemical weapon during World War I [509]. In addition, it has a strong greenhouse potential [510]. This molecule was detected in the atmosphere using solar occultation spectrometry [511], and using ACE-FTS [510] and MIPAS [512] retrievals.

Phosgene is a heavy molecule with small rotational constants and with a number of low-lying vibrational states. As a consequence, in order to perform a detailed and extensive analysis of its absorption region it is necessary to record spectra at low temperature. The analysis of the $11.75-\mu \mathrm{m}$ spectral region of phosgene (which is now in HITRAN2016) was performed using a highresolution Fourier transform spectrum recorded at $169 \mathrm{~K}$ [513]. The $v_{5}$ bands of the two isotopologues $\mathrm{CO}^{35} \mathrm{Cl}_{2}$ and $\mathrm{CO}^{35} \mathrm{Cl}^{37} \mathrm{Cl}$ were assigned up to very high quantum numbers and the corresponding upper-state ro-vibrational levels were fitted to within the experimental accuracy $\left(\sim 0.0002 \mathrm{~cm}^{-1}\right)$ [513]. On the other hand, it was not possible to assign the corresponding hot bands, which were modeled using extrapolated Hamiltonian constants. As a consequence, their line positions are not accurate. As far as the line intensities are concerned, they were calculated [512] using single transition moments calibrated using the cross-section measurements from Sharpe et al. [314]. Their accuracy is estimated to be on the order of $5 \%$.

\section{Absorption cross-sections}

Apart from the line-by-line spectroscopic absorption parameters discussed in Section 2, the HITRAN database contains information on absorption cross-sections where the line-by-line parameters are absent or incomplete. Usually this takes place for heavy polyatomic molecules (with low-lying vibrational modes) which are difficult for detailed analysis due to the high density of the spectral bands/lines, broadening effects, isomerization, and overall modeling complexity. Knowledge of the spectroscopy of these molecules is important for many purposes, including atmospheric remote sensing, radiative forcing calculations, detection of biomass burning, detection of toxic industrial chemicals (TICs), climate change monitoring, modeling planetary atmospheres, spectral calibration, etc. In fact these cross-sections have been applied in radiativetransfer codes in good approximations to the full-blown simulations that are possible with the line-by-line parameters [514-518]. It is therefore important to provide these cross-sections in HITRAN 
covering wide spectral ranges and at diverse thermodynamic conditions.

The cross-section files are provided in the HITRAN format described in Ref. [449] and are further described on the official HITRAN website (http://hitran.org/docs/cross-sections-definitions/). Each cross-section is given in a separate file with a header containing molecule name, chemical formula, wavenumber range, temperature, pressure, broadener, resolution of the experiment, and source. The file is completed with the value of each crosssection at equal intervals within the range, given in units of $\mathrm{cm}^{2}$ molecule ${ }^{-1}$. The cross-sections come from high-resolution laboratory observations, predominantly from Fourier transform spectrometers. Some of these measurements include small negative values, which for the HITRAN-formatted cross-sections were substituted by zeroes; in these cases we provide all the original crosssections in a supplementary "Alternate" folder, because the negative values provide the user with some information about the noise level in the measurements.

The HITRAN2012 edition of the database contained crosssections for about fifty gases. For the HITRAN2016 edition we have increased the amount of gases almost six fold by adding new data in the infrared spectral range. A dedicated paper detailing this update will be published separately. Here we only provide a brief summary and overview tables. Around 220 molecules of atmospheric, industrial, and remote sensing interests were taken from the quantitative spectroscopy PNNL (Pacific Northwest National Laboratory) database, described in Sharpe et al. [314]. This compilation was updated with 55 molecules from more recent work of Johnson et al. [519]. Twelve of these 55 molcules are new to PNNL. Spectra for these molecules will be indispensable, particularly for biomass burning detection. The PNNL database contains calibration-quality composite spectra given at atmospheric pressure and three temperatures $\left(5{ }^{\circ} \mathrm{C}, 25^{\circ} \mathrm{C}\right.$, and $\left.50{ }^{\circ} \mathrm{C}\right)$. All these cross-sections were measured using the FT-IR technique with $0.112 \mathrm{~cm}^{-1}$ spectral resolution on a Bruker IFS 66v/S spectrometer.

In addition, a large portion of new molecules of atmospheric interest was taken from the 2013 review by Hodnebrog et al. [520]. The data presented in this review are more varied than PNNL considering spectral resolution, while containing all the strong spectral features important in radiative forcing calculations (on Earth). The subset of air and nitrogen broadened cross-sections from this review is for 700-750 Torr pressure range and room temperature. Some lower-temperature data for $253 \mathrm{~K}$ from Highwood and Shine [521] were also included into HITRAN2016. Finally, more recently measured data were taken from Wagner and Birk for $\mathrm{BrONO}_{2}$ [522], Harrison et al. for CFC-12 [523], HCFC-22 [524], CCl 4 [525], HFC-23 [526], HFC-134a [527], Reed and Hodges (2015) for ethane [528], as well as newly measured and revised data for many molecules from Refs. [529-540].

The summary on the new and updated compounds divided by categories is presented in Table 9. These categories are implemented in HITRANonline for the convenience of users to browse the data. This summary does not include those compounds that were not updated. For instance, there are no new UV cross-sections that were added; therefore molecules that had only UV crosssections in HITRAN2012 are not included in this table.

In Fig. 27 we show an example of the update for the acetaldehyde molecule $\left(\mathrm{CH}_{3} \mathrm{CHO}\right)$. The new data were taken from the PNNL database [314], while the data from HITRAN2012 were originally from Tereszchuk and Bernath [541]. Sixteen cross-section temperature-pressure sets from Ref. [541] are presented in HITRAN2012 in the $2400-3400 \mathrm{~cm}^{-1}$ spectral range. These crosssections were calibrated using the PNNL data [541] and thus are in a good agreement with the update, in particular for absorption between $6.0 \times 10^{-21}$ and $1.0 \times 10^{-19} \mathrm{~cm}^{2}$ molecule ${ }^{-1}$. For this molecule, the new update extends the temperature range up to
$323 \mathrm{~K}$ and the wavenumber range from 510 to $6500 \mathrm{~cm}^{-1}$. The update includes stronger features in the mid-infrared which were absent in HITRAN2012. The data from Ref. [541] are also retained in the new HITRAN release.

In Table 10 the updates to the cross-sections of the molecules already existing in HITRAN2012 are presented. High-quality data were taken from different sources for the ranges which were either absent or covered insufficiently. Updates for molecules that were not previously in HITRAN are presented in Table 11. In both Table 10 and Table 11 we give total ranges for the wavenumber, pressure, temperature, and resolution. Each line in both of the tables summarizes all updated cross-sections for the given molecule, including those relegated to the alternate folder. If the broadener is not provided, self-broadening is assumed. Both tables contain total pressure of the sample including the buffer gas (where applicable).

While the extent of the update of the cross-sectional part of the database is impressive, this section of the database is by no means complete. In the future we plan further revision of high-quality data that become available in the literature and by the facilitation of new experiments. In the near future we also plan to extend the database to include planetary-relevant cross-sections that recently became available, including those given in Refs. [548-552]. An extensive update of the UV cross-sections is also planned.

\section{Collision-induced absorption}

Collision-induced absorption (CIA) was first introduced into HITRAN in the 2012 edition and was largely based on the data collected in Richard et al. [586]. This section of the database is accessible through (www.hitran.org/cia). CIA refers to absorption by transient electric dipoles induced by the interaction between colliding molecules. Due to the short life-time of collision complexes, this typically leads to broad absorption features underlying the much sharper lines due to absorption by isolated molecules. As CIA arises from binary collision complexes, the intensity scales with the number densities of both the absorber and the perturber, i.e., with the square of the pressure at constant volume mixing ratio. To be completely explicit, the transmittance at wavenumber $v$, $\tau_{\text {abs-pert }}(\nu)$, is related to the collision-induced cross section tabulated in the HITRAN CIA section, $\mathrm{k}_{\mathrm{abs} \text {-pert }}(v)$, through

$-\ln \left[\tau_{\text {abs-pert }}(v)\right]=\mathrm{k}_{\text {abs-pert }}(v) \rho_{\text {abs }} \rho_{\text {pert }} L$,

where $\rho_{\mathrm{abs}}$ and $\rho_{\text {pert }}$ are the number densities of the absorber and perturber, respectively, and $L$ is the path length. The CIA cross sections tabulated in HITRAN are given in $\mathrm{cm}^{5}$ molecule ${ }^{-2}$ throughout [586].

Often the relevant perturber will be air, i.e., a 21:79 mixture of $\mathrm{O}_{2}: \mathrm{N}_{2}$ (the amount of argon in the atmosphere coupled with its poor efficiency as a collisional partner makes this assumption quite reasonable). In that case, one can either add absorber- $\mathrm{O}_{2}$ and absorber- $\mathrm{N}_{2}$ contributions, or equivalently use the absorber-Air files which are introduced in this version of HITRAN. In the latter case, the perturber number density equals the air number density given by $\rho_{\text {pert }}=\rho_{\text {Air }}=\rho_{\mathrm{O} 2}+\rho_{\mathrm{N} 2}$.

\subsection{Molecular oxygen $1.27-\mu m$ band}

The CIA data for the 1.27- $\mu \mathrm{m}$ band (corresponding to $X^{3} \Sigma_{g}^{-}(\mathrm{v}$ $\left.=0) \rightarrow a^{1} \Delta_{\mathrm{g}}(\mathrm{v}=0)\right)$ of $\mathrm{O}_{2}$ have been revised in the current release of HITRAN. In this region, CIA is stronger whereas magnetic dipole lines are weaker when compared to the oxygen A-band. Hence, under atmospheric conditions, CIA contributes significantly to the absorption in the $1.27-\mu \mathrm{m}$ band.

All data included for this transition are taken from the experiment by Maté et al. [587], recorded using a Fourier transform spectrometer. Data recorded for pure $\mathrm{O}_{2}$ can be found in the $\mathrm{O}_{2}-\mathrm{O}_{2}$ 
Table 9

Categorization for the molecules in the HITRAN2016 update.

\begin{tabular}{llll}
\hline Category & $\mathrm{N}_{\text {src }}$ & $\mathrm{N}_{\text {mol }}$ & $\mathrm{N}_{\text {sets }}$ \\
\hline Halogenated alcohols and ethers & 27 & 63 & 106 \\
Hydrochlorofluorocarbons & 7 & 17 & 84 \\
Hydrofluorocarbons & 17 & 32 & 128 \\
Fully fluorinated species & 12 & 21 & 64 \\
Chlorocarbons and hydrochlorocarbons & 4 & 23 & 104 \\
Chlorofluorocarbons & 5 & 11 & 63 \\
Bromocarbons, hydrobromocarbons, and halons & 4 & 12 & 99 \\
Hydrocarbons & 3 & 30 & 80 \\
Alcohols, ethers and other oxygenated hydrocarbons & 2 & 76 & 62 \\
Nitriles, amines and other nitrogenated hydrocarbons & 3 & 24 & 35 \\
Sulfur-containing species & 3 & 13 & 8 \\
Iodocarbons and hydroiodocarbons & 1 & 3 & 5 \\
Other molecules & 2 & 2 & \\
\hline
\end{tabular}

Note: $\mathrm{N}_{\mathrm{src}}$ is the number of sources (references), $\mathrm{N}_{\text {mol }}$ is the number of molecules in the category (some molecules can be present in more than one category), and $\mathrm{N}_{\text {sets }}$ is the number of temperature-pressure sets.

\section{IR spectra for acetaldehyde}
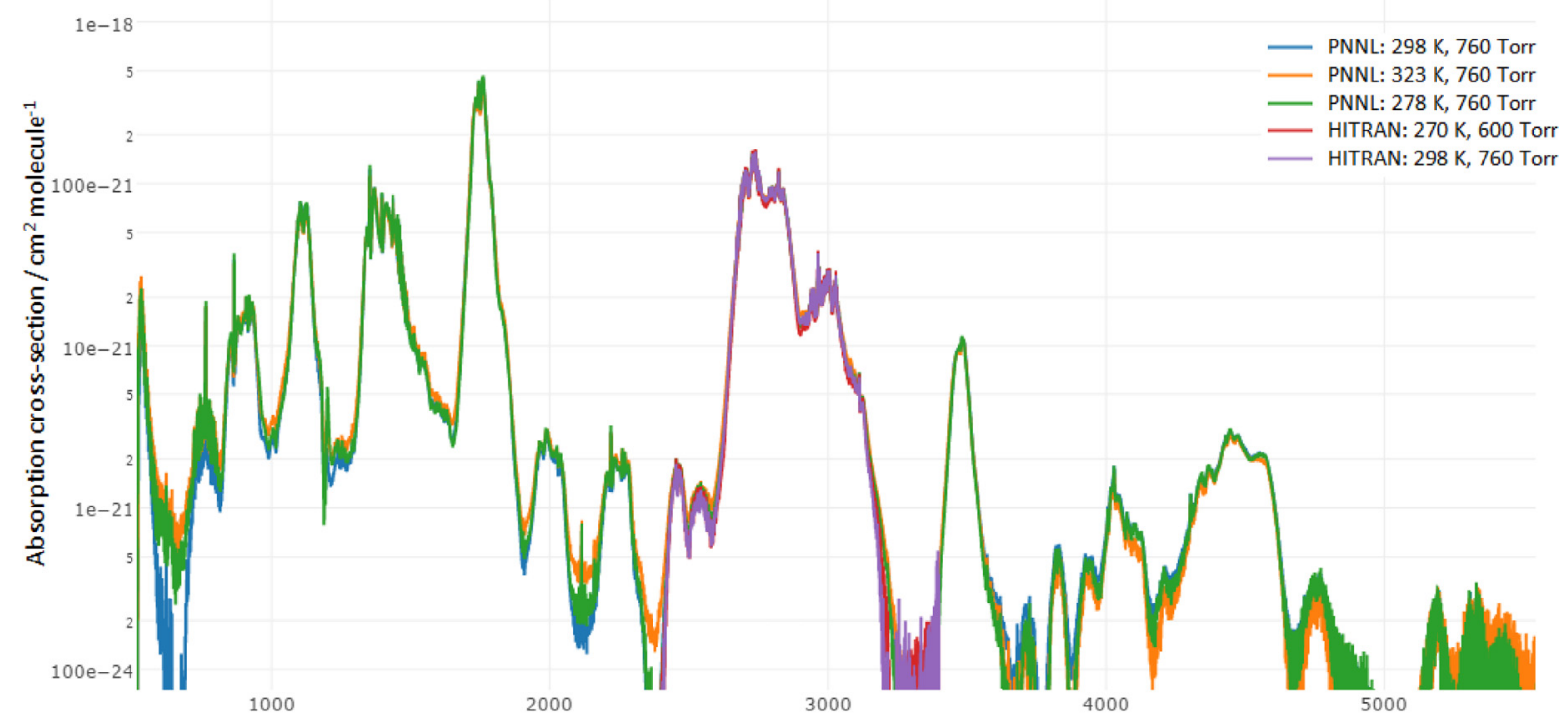

Wavenumber $/ \mathrm{cm}^{-1}$

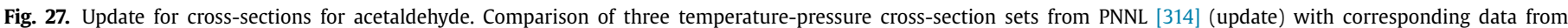
HITRAN2012 (Ref. [541]).

file. These data have been corrected for an error in HITRAN2012, which accidentally contained $\mathrm{O}_{2}$-Air spectra. Data recorded for air mixtures of 21:79 $\mathrm{O}_{2}: \mathrm{N}_{2}$ can be found in the $\mathrm{O}_{2}$-Air file, which is described above. In the previous version of HITRAN, data for such mixtures could be found in the $\mathrm{O}_{2}-\mathrm{N}_{2}$ file, but these have been moved to the $\mathrm{O}_{2}$-Air file to stress that these also include $\mathrm{O}_{2}-\mathrm{O}_{2}$ contributions. One should not double count the $\mathrm{O}_{2}-\mathrm{O}_{2}$ CIA by including both $\mathrm{O}_{2}-\mathrm{O}_{2}$ and $\mathrm{O}_{2}$-Air contributions in atmospheric models. This has replaced the data of Smith and Newnham for this band [588]. The data previously provided in this band for $\mathrm{O}_{2}: \mathrm{N}_{2}$ mixtures contained magnetic dipole lines due to $\mathrm{O}_{2}$ monomer absorption. This issue has now been resolved, as the monomer lines have been subtracted from the present data.

\subsection{Oxygen $1.06-\mu m$ band in air}

The molecular oxygen $X^{3} \Sigma_{g}^{-}(\mathrm{v}=0) \rightarrow a^{1} \Delta_{\mathrm{g}}(\mathrm{v}=1)$ transition at $1.06 \mu \mathrm{m}$ has much weaker magnetic dipole lines than what is observed for the $1.27-\mu \mathrm{m}$ band, and hence the $1.06-\mu \mathrm{m}$ band is dominated by collision-induced absorption. The previous version of HITRAN already contained spectra for this transition, for different $\mathrm{O}_{2}: \mathrm{N}_{2}$ mixtures, measured by Smith and Newnham [588].
These spectra have been re-analyzed by fitting spectra for different mixtures to contributions of $\mathrm{O}_{2}-\mathrm{O}_{2}$ and $\mathrm{O}_{2}-\mathrm{N}_{2}$ collisional pairs. This is illustrated in Fig. 28, which shows two spectra, for 50:50 and 75:25 mixtures of $\mathrm{O}_{2}: \mathrm{N}_{2}$, both at $T=230 \mathrm{~K}$. These spectra can be reproduced essentially using only the $\mathrm{O}_{2}-\mathrm{O}_{2}$ contribution as shown. The contribution of $\mathrm{O}_{2}-\mathrm{N}_{2}$ is smaller than the error bars and noise levels. The spectra determined in this way have lower noise levels than direct measurements for air mixtures, where the absorption is weaker due to the lower $\mathrm{O}_{2}$ density. These data have been smoothed further and are now available in the $\mathrm{O}_{2}$-Air file.

\subsection{Oxygen $A$ band in air}

Collision-induced absorption in the oxygen A band is also of atmospheric interest. In spite of the smaller contribution of CIA in this band, relative to the much stronger magnetic dipole lines, CIA is significant for high-accuracy measurements including airmass calibration of the OCO-2 instrument [589]. The HITRAN CIA database already contained data for $\mathrm{O}_{2}-\mathrm{O}_{2}$ and $\mathrm{O}_{2}-\mathrm{N}_{2}$ from Tran et al. [253], valid for temperatures between 200 and $300 \mathrm{~K}$. For 
Table 10

Summary for the updates for compounds already existing in HITRAN2012.

\begin{tabular}{|c|c|c|c|c|c|c|c|}
\hline Molecule & $\mathrm{CAS}^{*}$ & $\begin{array}{l}\text { Wavenumber } \\
\text { range }\left(\mathrm{cm}^{-1}\right)\end{array}$ & $\mathrm{T}(\mathrm{K})$ & $\mathrm{P}$ (Torr) & $\begin{array}{l}\text { Resolution } \\
\left(\mathrm{cm}^{-1}\right)\end{array}$ & Broadener & Reference \\
\hline Acetylaldehyde & $75-07-0$ & $510-6500$ & $278-323$ & 760 & 0.112 & $\mathrm{~N}_{2}$ & Sharpe et al. (2004) [314] \\
\hline Bromine nitrate & $40423-14-1$ & $765-1320$ & $204-293$ & $0-141$ & 0.014 & air & Wagner and Birk (2016) [522] \\
\hline $\begin{array}{l}\text { Carbon } \\
\text { tetrachloride }\end{array}$ & $56-23-5$ & $700-860$ & $207-297$ & $7-760$ & $0.01-0.112$ & air & $\begin{array}{l}\text { Harrison et al. JQSRT (2016) [525], Wallington } \\
\text { et al. (2016) [540]* }\end{array}$ \\
\hline CFC-11 & $75-69-4$ & $570-6500$ & $278-323$ & $700-760$ & $0.112-0.5$ & $\mathrm{~N}_{2}$, air & $\begin{array}{l}\text { Sharpe et al. (2004) [314], Sihra et al. (2001) } \\
\text { [542]* }\end{array}$ \\
\hline CFC-113 & $76-13-1$ & $620-5000$ & $278-323$ & 760 & 0.112 & $\mathrm{~N}_{2}$ & Sharpe et al. (2004) [314] \\
\hline CFC-114 & $76-14-2$ & $600-5000$ & $278-323$ & 760 & 0.112 & $\mathrm{~N}_{2}$ & Sharpe et al. (2004) [314] \\
\hline CFC-115 & $76-15-3$ & $525-6500$ & $278-323$ & 760 & 0.112 & $\mathrm{~N}_{2}$ & Sharpe et al. (2004) [314] $]^{*}$ \\
\hline CFC-12 & $75-71-8$ & $800-1270$ & $189-295$ & $7-761$ & $0.01-0.03$ & air & Harrison AMT (2015) [523] \\
\hline CFC-13 & $75-72-9$ & $600-6500$ & $278-323$ & 760 & 0.112 & $\mathrm{~N}_{2}$ & Sharpe et al. (2004) [314]* \\
\hline Ethane & $74-84-0$ & $600-6500$ & $278-323$ & 760 & 0.112 & $\mathrm{~N}_{2}$ & $\begin{array}{l}\text { Sharpe et al. (2004) [314]*, Reed et al. (2015) } \\
\text { [528] }\end{array}$ \\
\hline Ethylene & $74-85-1$ & $600-6500$ & $278-323$ & 760 & 0.112 & $\mathrm{~N}_{2}$ & Sharpe et al. (2004) [314]* \\
\hline Formaldehyde & $50-00-0$ & $600-6500$ & $278-323$ & 760 & 0.112 & $\mathrm{~N}_{2}$ & Sharpe et al. (2004) [314]* \\
\hline Formic acid & $64-18-6$ & $540-7200$ & $298-323$ & 760 & 0.112 & $\mathrm{~N}_{2}$ & Sharpe et al. (2004) [314]* \\
\hline HCFC-123 & $306-83-2$ & $400-6500$ & $278-323$ & $700-760$ & $0.112-0.5$ & $\mathrm{~N}_{2}$, air & $\begin{array}{l}\text { Sharpe et al. (2004) [314], Sihra et al. (2001) } \\
\text { [542]* }\end{array}$ \\
\hline HCFC-124 & 2837-89-0 & $400-6500$ & $278-323$ & $700-760$ & $0.112-0.5$ & $\mathrm{~N}_{2}$, air & $\begin{array}{l}\text { Sharpe et al. (2004) [314], Sihra et al. (2001) } \\
\text { [542]* }\end{array}$ \\
\hline HCFC-141b & $1717-00-6$ & $500-6500$ & $278-323$ & $700-760$ & $0.112-0.5$ & $\mathrm{~N}_{2}$, air & $\begin{array}{l}\text { Sharpe et al. (2004) [314], Sihra et al. (2001) } \\
\text { [542]* }\end{array}$ \\
\hline HCFC142b & $75-68-3$ & $500-6500$ & $278-323$ & $700-760$ & $0.112-0.5$ & $\mathrm{~N}_{2}$, air & $\begin{array}{l}\text { Sharpe et al. (2004) [314], Sihra et al. (2001) } \\
\text { [542]* }\end{array}$ \\
\hline HCFC-21 & $75-43-4$ & $600-7300$ & $278-323$ & $700-760$ & $0.112-0.5$ & $\mathrm{~N}_{2}$, air & $\begin{array}{l}\text { Sharpe et al. (2004) [314], Sihra et al. (2001) } \\
\text { [542]* }\end{array}$ \\
\hline HCFC-22 & $75-45-6$ & $720-1380$ & $190-295$ & $7-762$ & $0.01-0.03$ & air & Harrison AMT (2016) [524] \\
\hline HCFC-225ca & $422-56-0$ & $650-1400$ & 296 & 700 & 0.5 & air & Sihra et al. (2001) [542] \\
\hline HCFC-225cb & $507-55-1$ & $700-1400$ & 296 & 700 & 0.5 & air & Sihra et al. (2001) [542] \\
\hline Hexafluoroethane & $76-16-4$ & $400-6500$ & $253-323$ & $0-760$ & $0.01-0.5$ & $\mathrm{~N}_{2}$, air, self & $\begin{array}{l}\text { Highwood and Shine (2000) [521], Bravo et al. } \\
\text { (2010b) [543], Sihra et al. (2001) [542]*, } \\
\text { Sharpe et al. (2004) [314] }\end{array}$ \\
\hline HFC-125 & $354-33-6$ & $450-6500$ & $278-323$ & $700-760$ & $0.112-0.5$ & $\mathrm{~N}_{2}$, air & $\begin{array}{l}\text { Young et al. (2009b) [544], Sharpe et al. (2004) } \\
\text { [314], Sihra et al. (2001) [542]* }\end{array}$ \\
\hline HFC-134 & $359-35-3$ & $350-6500$ & $278-323$ & $700-760$ & $0.112-0.5$ & $\mathrm{~N}_{2}$, air & $\begin{array}{l}\text { Sharpe et al. (2004) [314], Sihra et al. (2001) } \\
\text { [542]* }\end{array}$ \\
\hline HFC-134a & $811-97-2$ & $750-1600$ & $190-296$ & $22-761$ & 0.015 & air & Harrison JQSRT (2015) [527] \\
\hline HFC-143a & $420-46-2$ & $500-6500$ & $253-323$ & $0-760$ & $0.03-0.5$ & $\mathrm{~N}_{2}$, air, self & $\begin{array}{l}\text { Highwood and Shine (2000) [521]*, Sharpe et } \\
\text { al. (2004) [314], Sihra et al. (2001) [542]*, Le } \\
\text { Bris and Graham. JQSRT (2015) [535] }\end{array}$ \\
\hline HFC-152a & $75-37-6$ & $400-6500$ & $253-323$ & $0-760$ & $0.03-0.5$ & $\mathrm{~N}_{2}$, air, self & $\begin{array}{l}\text { Highwood and Shine (2000) [521], Sharpe et al. } \\
\text { (2004) [314], Sihra et al. (2001) [542]* }\end{array}$ \\
\hline HFC-32 & $75-10-5$ & $400-6500$ & $253-323$ & $0-760$ & $0.03-0.5$ & $\mathrm{~N}_{2}$, air, self & $\begin{array}{l}\text { Highwood and Shine (2000) [521], Sharpe et al. } \\
\text { (2004) [314], Gohar et al. (2004) [545], }\end{array}$ \\
\hline Hydrogen peroxide & $7722-84-1$ & $510-7500$ & $298-323$ & 760 & 0.112 & $\mathrm{~N}_{2}$ & Johnson et al. (2010) [519]* \\
\hline Methylbromide & $74-83-9$ & $500-6500$ & $278-323$ & $700-760$ & $0.112-0.5$ & $\mathrm{~N}_{2}$, air & Sharpe et al. (2004) [314]*, \\
\hline Phosphine & $7803-51-2$ & $600-6500$ & $278-323$ & 760 & 0.112 & $\mathrm{~N}_{2}$ & Sharpe et al. (2004) [314]* \\
\hline Propane & $74-98-6$ & $600-6500$ & $278-323$ & 760 & 0.112 & $\mathrm{~N}_{2}$ & Sharpe et al. (2004) [314] \\
\hline Tetrafluoromethane & $75-73-0$ & $500-6500$ & $278-323$ & $700-760$ & $0.112-0.5$ & $\mathrm{~N}_{2}$, air & $\begin{array}{l}\text { Sharpe et al. (2004) [314], Hurley et al. (2005) } \\
\text { [546]* }\end{array}$ \\
\hline \multirow[t]{2}{*}{ Toluene } & $108-88-3$ & $600-6500$ & $278-323$ & 760 & 0.112 & $\mathrm{~N}_{2}$ & Sharpe et al. (2004) [314] \\
\hline & $373-80-8$ & $100-6500$ & $278-323$ & 760 & $0.112-0.9$ & $\mathrm{~N}_{2}$ & Nielsen et al. (2002) [547]* \\
\hline \multicolumn{8}{|c|}{$\begin{array}{l}\text { Trifluoromethylsulfur } \\
\text { pentafluoride }\end{array}$} \\
\hline m-Xylene & $108-38-3$ & $580-6500$ & $298-323$ & 760 & 0.112 & $\mathrm{~N}_{2}$ & Sharpe et al. (2004) [314] \\
\hline o-Xylene & $95-47-6$ & $600-6500$ & $298-323$ & 760 & 0.112 & $\mathrm{~N}_{2}$ & Sharpe et al. (2004) [314] \\
\hline p-Xylene & $106-42-3$ & $580-6500$ & $298-323$ & 760 & 0.112 & $\mathrm{~N}_{2}$ & Sharpe et al. (2004) [314] \\
\hline
\end{tabular}

- Chemical Abstracts Service number

*Data from these sources can be found in the alternate folder.

convenience, we now also provide the spectrum for $\mathrm{O}_{2}-\mathrm{Air}$, obtained by combining the available $\mathrm{O}_{2}-\mathrm{O}_{2}$ and $\mathrm{O}_{2}-\mathrm{N}_{2}$ data. In the alternate folder, we now also include $\mathrm{O}_{2}$-Air data from Drouin et al. [252] obtained in a multispectrum fitting approach. Line-byline parameters determined in the same multispectrum fit, are also available in HITRAN2016. Consistent use of both line-by-line and CIA data accurately reproduces the total absorption under atmospheric conditions, and has been shown to reduce systematic errors in atmospheric retrievals [252].

\subsection{Planned update}

This update represents a small revision of the HITRAN CIA section, and we aim to extend the CIA section with recently measured and calculated spectra in the near future. This update will include existing and new collisional pairs. We also plan to further update different spectral regions for molecular oxygen. 


\section{Aerosol refractive indices}

Optical depths in the atmosphere are determined by the spatial and temporal distributions of gases, clouds, and aerosols [590]. Clouds and aerosols also take part in chemical reactions in both the liquid and solid phases [591]. The interpretation of remotesensing retrievals of gaseous species is limited frequently by how well one can separate gaseous opacity from that of clouds and aerosols. Knowledge of the refractive indices of the aerosols and cloud particles and their size distributions is necessary in order to specify their optical properties. HITRAN2016 contains refractive indices in the visible, infrared, and millimeter spectral ranges of many types of cloud and aerosol particles. Table 12 lists the HITRAN2016 indices.

As discussed by Bohren and Huffman [633], the refractive index $\mathrm{m}$ is a function of wavelength, and has a real, $\mathrm{m}_{\text {real }}$, and imaginary, $\mathrm{m}_{\mathrm{imag}}$, component:

$\mathrm{m}=\mathrm{m}_{\text {real }}+\mathrm{im}_{\mathrm{imag}}$

Table 11

Summary of the new compounds for which cross-sections were added in HITRAN.

\begin{tabular}{|c|c|c|c|c|c|c|c|}
\hline Molecule & CAS & $\begin{array}{l}\text { Wavenumber } \\
\text { range, } \mathrm{cm}^{-1}\end{array}$ & $\mathrm{~T}, \mathrm{~K}$ & P,Torr & Resolution, $\mathrm{cm}^{-1}$ & Broadener & Reference \\
\hline $\begin{array}{l}\text { (E)-1-Chloro-3,3,3- } \\
\text { trifluoroprop-1-ene }\end{array}$ & $102687-65-0$ & $600-2000$ & 295 & 700 & 0.25 & air & $\begin{array}{l}\text { Andersen et al. (2008) } \\
\text { [553] }\end{array}$ \\
\hline (E)-HFC-1225ye & $5595-10-8$ & $650-2000$ & 296 & 700 & 0.25 & air & Hurley et al. (2007) [554] \\
\hline $\begin{array}{l}\text { (Perfluoro-n-butyl)- } \\
\text { ethylene }\end{array}$ & $19430-93-4$ & 650-1999 & 298 & 700 & 0.25 & air & $\begin{array}{l}\text { Andersen et al. (2012b) } \\
\text { [555] }\end{array}$ \\
\hline (Perfluoro-n-octyl)-ethylene & $21652-58-4$ & 650-1999 & 298 & 700 & 0.25 & air & $\begin{array}{l}\text { Andersen et al. (2012b) } \\
\text { [555] }\end{array}$ \\
\hline (Z)-HFC-1225ye & $5528-43-8$ & $650-2000$ & 296 & 700 & 0.25 & air & Hurley et al. (2007) [554] \\
\hline (Z)-HFC-1234ze & $29118-25-0$ & $600-2000$ & 296 & 700 & 0.25 & air & Nilsson et al. (2009) [556] \\
\hline 1,1,1,2-Tetrachloro-ethane & $630-20-6$ & $530-6500$ & $278-323$ & 760 & 0.112 & $\mathrm{~N}_{2}$ & Sharpe et al. (2004) [314] \\
\hline 1,1,2,2-Tetrachloro ethane & $79-34-5$ & $530-6500$ & $278-323$ & 760 & 0.112 & $\mathrm{~N}_{2}$ & Sharpe et al. (2004) [314] \\
\hline $\begin{array}{l}\text { 1,1,2,2-Tetrafluoro-1,2- } \\
\text { dimethoxyethane }\end{array}$ & $73287-23-7$ & $150-1900$ & 296 & 700 & 0.25 & air & $\begin{array}{l}\text { Andersen et al. (2004) } \\
\text { [557] }\end{array}$ \\
\hline $\begin{array}{l}\text { 1,1,2,2-Tetrafluoro-3- } \\
\text { methoxypropane }\end{array}$ & $60598-17-6$ & $450-3200$ & 298 & & 1.0 & & Oyaro et al. (2004) [558] \\
\hline 1,1,2-Trichloro-ethane & $79-00-5$ & $600-6500$ & $278-323$ & 760 & 0.112 & $\mathrm{~N}_{2}$ & Sharpe et al. (2004) [314] \\
\hline $\begin{array}{l}\text { 1,1,2-Trifluoro-2- } \\
\text { (trifluoromethoxy)- } \\
\text { ethane }\end{array}$ & $84011-06-3$ & $440-3200$ & 298 & & 1.0 & & Oyaro et al. (2005) [559] \\
\hline 1,1-Dichloroethane & $75-34-3$ & $560-6500$ & $278-323$ & 760 & 0.112 & $\mathrm{~N}_{2}$ & Sharpe et al. (2004) [314] \\
\hline 1,2-Dichloropropane & $78-87-5$ & $590-6500$ & $278-323$ & 760 & 0.112 & $\mathrm{~N}_{2}$ & Sharpe et al. (2004) [314] \\
\hline 1,2-Diclorobenzene & $95-50-1$ & $600-6500$ & $278-323$ & 760 & 0.112 & $\mathrm{~N}_{2}$ & Sharpe et al. (2004) [314] \\
\hline $\begin{array}{l}\text { 1,2-Difluoro-1,2- } \\
\text { dichloroethane }\end{array}$ & $431-06-1$ & $550-6500$ & $278-323$ & 760 & 0.112 & $\mathrm{~N}_{2}$ & Sharpe et al. (2004) [314] \\
\hline 1,3-Butadiene & $106-99-0$ & $530-6500$ & $278-323$ & 760 & 0.112 & $\mathrm{~N}_{2}$ & Sharpe et al. (2004) [314] \\
\hline 1,3-Dichlorobenzene & $541-73-1$ & $600-6500$ & $278-323$ & 760 & 0.112 & $\mathrm{~N}_{2}$ & Sharpe et al. (2004) [314] \\
\hline 1,3-Dichloropropane & $142-28-9$ & $570-6500$ & $278-323$ & 760 & 0.112 & $\mathrm{~N}_{2}$ & Sharpe et al. (2004) [314] \\
\hline 1,4-Dichlorobenzene & $106-46-7$ & $600-6500$ & $298-323$ & 760 & 0.112 & $\mathrm{~N}_{2}$ & Sharpe et al. (2004) [314] \\
\hline 1-Butene & $25167-67-3$ & $525-6500$ & $278-323$ & 760 & 0.112 & $\mathrm{~N}_{2}$ & Sharpe et al. (2004) [314] \\
\hline $\begin{array}{l}\text { 1-Ethoxy-1,1,2,3,3,3- } \\
\text { hexafluoropropane }\end{array}$ & $380-34-7$ & $400-3500$ & 298 & & 1.0 & & Oyaro et al. (2005) [559] \\
\hline $\begin{array}{l}\text { 1H,1H,2H,2H- } \\
\text { Perfluorononan-1-ol }\end{array}$ & $755-02-2$ & $500-2250$ & 296 & 700 & 0.5 & air & $\begin{array}{l}\text { Waterland et al. (2005) } \\
\text { [560] }\end{array}$ \\
\hline $\begin{array}{l}\text { 1H,1H,2H,2H- } \\
\text { Perfluoroundecan-1-ol }\end{array}$ & $87017-97-8$ & $600-2000$ & 296 & 700 & 0.5 & air & $\begin{array}{l}\text { Waterland et al. (2005) } \\
\text { [560] }\end{array}$ \\
\hline 1-Nonene & $124-11-8$ & $550-6500$ & $298-323$ & 760 & 0.112 & $\mathrm{~N}_{2}$ & Sharpe et al. (2004) [314] \\
\hline 1-Penten-3-ol & $616-25-1$ & $550-7300$ & $298-323$ & 760 & 0.112 & $\mathrm{~N}_{2}$ & Johnson et al. (2010) [519] \\
\hline 2,2,2-trifluoro-acetaldehyde & & $400-2500$ & $296-298$ & 700 & $0.5-1.0$ & air & $\begin{array}{l}\text { Sellevag et al. (2004a) } \\
\text { [561], Hashikawa et al. } \\
\text { (2004) [562]* }\end{array}$ \\
\hline 2,2,2-Trifluoroethanol & $75-89-8$ & $70-7250$ & $278-323$ & 760 & $0.112-1.0$ & $\mathrm{~N}_{2}$ & $\begin{array}{l}\text { Sharpe et al. (2004) [314], } \\
\text { Sellevag et al. (2004b) } \\
\text { [563] }\end{array}$ \\
\hline $\begin{array}{l}\text { 2,2,3,3,4,4,4- } \\
\text { Heptafluorobutan-1-ol }\end{array}$ & $375-01-9$ & $400-4001$ & 298 & & 0.01 & & $\begin{array}{l}\text { Sellevag et al. (2007) } \\
\text { [533]*, Bravo et al. } \\
\text { (2010a) [564] }\end{array}$ \\
\hline $\begin{array}{l}\text { 2,2,3,3-Tetrafluoro-1- } \\
\text { propanol }\end{array}$ & $76-37-9$ & $400-4000$ & 298 & & & & Sellevag et al. (2007) [533] \\
\hline $\begin{array}{l}\text { 2,2,3,4,4,4-Hexafluoro-1- } \\
\text { butanol }\end{array}$ & $382-31-0$ & $400-4000$ & 298 & & & & Sellevag et al. (2007) [533] \\
\hline 2,2-Difluoroethanol & $359-13-7$ & $70-4800$ & 293 & & 1.0 & & $\begin{array}{l}\text { Sellevag et al. (2004b) } \\
\text { [563] }\end{array}$ \\
\hline 2,3-Butanedione & $431-03-8$ & $580-6500$ & $278-323$ & 760 & 0.112 & $\mathrm{~N}_{2}$ & Johnson et al. (2010) [519] \\
\hline 2,3-Dimethylfuran & 14920-89-9 & $510-6500$ & $278-323$ & 760 & 0.112 & $\mathrm{~N}_{2}$ & Johnson et al. (2010) [519] \\
\hline 2,5-Dimethylfuran & $625-86-5$ & $550-7000$ & $278-323$ & 760 & 0.112 & $\mathrm{~N}_{2}$ & Johnson et al. (2010) [519] \\
\hline 2,6-Dimethoxyphenol & $91-10-1$ & $580-6500$ & 323 & 760 & 0.112 & $\mathrm{~N}_{2}$ & Johnson et al. (2010) [519] \\
\hline 2-Carene & $554-61-0$ & $600-7300$ & $298-323$ & 760 & 0.112 & $\mathrm{~N}_{2}$ & Johnson et al. (2010) [519] \\
\hline 2-Ethyltoluene & $611-14-3$ & $520-6500$ & $298-323$ & 760 & 0.112 & $\mathrm{~N}_{2}$ & Sharpe et al. (2004) [314] \\
\hline 2-Fluoroethanol & $371-62-0$ & $80-7250$ & $293-323$ & 760 & $0.112-1.0$ & $\mathrm{~N}_{2}$ & $\begin{array}{l}\text { Sharpe et al. (2004) [314], } \\
\text { Sellevag et al. (2004b) } \\
\text { [563] }\end{array}$ \\
\hline
\end{tabular}


Table 11 (continued)

\begin{tabular}{|c|c|c|c|c|c|c|c|}
\hline Molecule & CAS & $\begin{array}{l}\text { Wavenumber } \\
\text { range, } \mathrm{cm}^{-1}\end{array}$ & $\mathrm{~T}, \mathrm{~K}$ & P,Torr & Resolution, $\mathrm{cm}^{-1}$ & Broadener & Reference \\
\hline 2H, 3H-Perfluoropentane & $138495-42-8$ & $500-6500$ & $278-323$ & 760 & 0.112 & $\mathrm{~N}_{2}$ & Sharpe et al. (2004) [314] \\
\hline 2-Hexanol & $626-93-7$ & $520-7250$ & $298-323$ & 760 & 0.112 & $\mathrm{~N}_{2}$ & Johnson et al. (2010) [519] \\
\hline 2-Methylfuran & $534-22-5$ & $550-6500$ & $278-323$ & 760 & 0.112 & $\mathrm{~N}_{2}$ & Johnson et al. (2010) [519] \\
\hline 2-Nonanone & $821-55-6$ & $550-6500$ & $298-323$ & 760 & 0.112 & $\mathrm{~N}_{2}$ & Johnson et al. (2010) [519] \\
\hline 2-Pentylfuran & $3777-69-3$ & $550-7300$ & $298-323$ & 760 & 0.112 & $\mathrm{~N}_{2}$ & Johnson et al. (2010) [519] \\
\hline 2-Vinylpyridine & $100-69-6$ & $540-7300$ & $298-323$ & 760 & 0.112 & $\mathrm{~N}_{2}$ & Johnson et al. (2010) [519] \\
\hline 3,3,3-Trifluoropropanal & $460-40-2$ & $400-3500$ & 298 & & 1.0 & & $\begin{array}{l}\text { Sellevag et al. (2004a) } \\
\text { [561] }\end{array}$ \\
\hline $\begin{array}{l}\text { 3,3,4,4,5,5,6,6,7,7,7- } \\
\text { Undecafluoro-heptan-1-ol }\end{array}$ & $185689-57-0$ & $600-2000$ & 296 & 700 & 0.5 & air & $\begin{array}{l}\text { Waterland et al. (2005) } \\
\text { [560] }\end{array}$ \\
\hline 3,3-Demethyl-2-pentanol & $19781-24-9$ & $520-7250$ & $298-323$ & 760 & 0.112 & $\mathrm{~N}_{2}$ & Johnson et al. (2010) [519] \\
\hline 3-Carene & $13466-78-9$ & $600-7300$ & $298-323$ & 760 & 0.112 & $\mathrm{~N}_{2}$ & Johnson et al. (2010) [519] \\
\hline 3-Methoxyphenol & $150-19-6$ & $550-6500$ & $323-333$ & 760 & 0.112 & $\mathrm{~N}_{2}$ & Johnson et al. (2010) [519] \\
\hline 3-Methylfuran & $930-27-8$ & $550-6500$ & $278-323$ & 760 & 0.112 & $\mathrm{~N}_{2}$ & Johnson et al. (2010) [519] \\
\hline 3-Pentanol & $584-02-1$ & $570-7250$ & $298-323$ & 760 & 0.112 & $\mathrm{~N}_{2}$ & Johnson et al. (2010) [519] \\
\hline 4-Ethyltoluene & $622-96-8$ & $600-6500$ & $298-323$ & 760 & 0.112 & $\mathrm{~N}_{2}$ & Sharpe et al. (2004) [314] \\
\hline 4-Methyl-1-pentanol & $626-89-1$ & $520-7250$ & $298-323$ & 760 & 0.112 & $\mathrm{~N}_{2}$ & Johnson et al. (2010) [519] \\
\hline 4-Methylvaleric acid & $646-07-1$ & $500-7300$ & $323-338$ & 760 & 0.112 & $\mathrm{~N}_{2}$ & Johnson et al. (2010) [519] \\
\hline 4-Penten-1-ol & $821-09-0$ & $520-7400$ & $298-323$ & 760 & 0.112 & $\mathrm{~N}_{2}$ & Johnson et al. (2010) [519] \\
\hline 5-Nonanol & $623-93-8$ & $580-7200$ & $298-323$ & 760 & 0.112 & $\mathrm{~N}_{2}$ & Johnson et al. (2010) [519] \\
\hline Acetic acid & $64-19-7$ & $550-7100$ & $298-323$ & 760 & 0.112 & $\mathrm{~N}_{2}$ & Sharpe et al. (2004) [314] \\
\hline Acetol & $116-09-6$ & $435-7200$ & $298-323$ & 760 & 0.112 & $\mathrm{~N}_{2}$ & Johnson et al. (2010) [519] \\
\hline Acrolein & $107-02-8$ & $540-6500$ & $278-323$ & 760 & 0.112 & $\mathrm{~N}_{2}$ & Sharpe et al. (2004) [314] \\
\hline Acrylic acid & $79-10-7$ & $525-6500$ & 323 & 760 & 0.112 & $\mathrm{~N}_{2}$ & Johnson et al. (2010) [519] \\
\hline Acrylonitrile & $107-13-1$ & $550-6500$ & $278-323$ & 760 & 0.112 & $\mathrm{~N}_{2}$ & Sharpe et al. (2004) [314] \\
\hline Allyl trifluoroacetate & $383-67-5$ & $600-2000$ & 298 & & 0.03 & air & $\begin{array}{l}\text { Rodrigues et al. (2016) } \\
\text { [538] }\end{array}$ \\
\hline alpha-Pinene (1S) (-) & $7785-26-4$ & $600-6500$ & $298-323$ & 760 & 0.112 & $\mathrm{~N}_{2}$ & Sharpe et al. (2004) [314] \\
\hline Benzaldehyde & $100-52-7$ & $600-6500$ & $278-323$ & 760 & 0.112 & $\mathrm{~N}_{2}$ & Sharpe et al. (2004) [314] \\
\hline Benzyl alcohol & $100-51-6$ & $550-7400$ & $298-323$ & 760 & 0.112 & $\mathrm{~N}_{2}$ & Sharpe et al. (2004) [314] \\
\hline beta-Pinene (1S) (-) & $18172-67-3$ & $600-6500$ & $298-323$ & 760 & 0.112 & $\mathrm{~N}_{2}$ & Sharpe et al. (2004) [314] \\
\hline bis (2-Chloroethyl) ether & $111-44-4$ & $550-6500$ & $298-323$ & 760 & 0.112 & $\mathrm{~N}_{2}$ & Sharpe et al. (2004) [314] \\
\hline Bromochloromethane & $74-97-5$ & $550-7100$ & $278-323$ & 760 & 0.112 & $\mathrm{~N}_{2}$ & Sharpe et al. (2004) [314] \\
\hline Bromoform & $75-25-2$ & $600-6500$ & $278-323$ & 760 & 0.112 & $\mathrm{~N}_{2}$ & Sharpe et al. (2004) [314] \\
\hline $\mathrm{C}_{2} \mathrm{Cl}_{3} \mathrm{H}_{3}$ & $71-55-6$ & $500-6500$ & $278-323$ & 760 & 0.112 & $\mathrm{~N}_{2}$ & Sharpe et al. (2004) [314] \\
\hline $\mathrm{C}_{4} \mathrm{H}_{5} \mathrm{~F}_{3} \mathrm{O}_{2}$ & $383-63-1$ & $500-6500$ & $278-323$ & 760 & 0.112 & $\mathrm{~N}_{2}$ & Sharpe et al. (2004) [314] \\
\hline Carbon disulfide & $75-15-0$ & $600-6500$ & $278-323$ & 760 & 0.112 & $\mathrm{~N}_{2}$ & Sharpe et al. (2004) [314] \\
\hline $\mathrm{CF}_{3} \mathrm{CH}_{2} \mathrm{CH}_{2} \mathrm{OH}$ & $2240-88-2$ & $400-4000$ & $296-298$ & 700 & 0.5 & air & $\begin{array}{l}\text { Waterland et al. (2005) } \\
\text { [560], Sellevag et al. } \\
(2007)[533]^{*}\end{array}$ \\
\hline CFC-112 & $76-12-0$ & $550-6500$ & $278-323$ & 760 & $0.112-1.0$ & $\mathrm{~N}_{2}$ & $\begin{array}{l}\text { Sharpe et al. (2004) [314], } \\
\text { Davis et al. (2016) [529]* }\end{array}$ \\
\hline CFC-112a & $76-11-9$ & $550-6500$ & $278-323$ & 760 & $0.112-1.0$ & $\mathrm{~N}_{2}$ & $\begin{array}{l}\text { Sharpe et al. (2004) [314], } \\
\text { Davis et al. (2016) [529]* }\end{array}$ \\
\hline CFC-113a & $354-58-5$ & $550-1730$ & $295-296$ & 2.7 & $0.01-1.0$ & & $\begin{array}{l}\text { Davis et al. (2016) [529]*, } \\
\text { Etminan et al. (2014) } \\
\text { [530] }\end{array}$ \\
\hline CFC-114a & $374-07-2$ & $550-1397$ & 296 & & 1.0 & & Davis et al. (2016) [529] \\
\hline Chloroacetone & $78-95-5$ & $600-6500$ & $278-323$ & 760 & 0.112 & $\mathrm{~N}_{2}$ & Sharpe et al. (2004) [314] \\
\hline Chlorobenzene & $108-90-7$ & $600-6500$ & $278-323$ & 760 & 0.112 & $\mathrm{~N}_{2}$ & Sharpe et al. (2004) [314] \\
\hline Chloroethane & $75-00-3$ & $590-6500$ & $278-323$ & 760 & 0.112 & $\mathrm{~N}_{2}$ & Sharpe et al. (2004) [314] \\
\hline Chloromethanol & & $657-2500$ & 295 & 700 & 0.25 & $\mathrm{~N}_{2}$ & $\begin{array}{l}\text { Wallington et al. (2000) } \\
\text { [565] }\end{array}$ \\
\hline Cineole & $470-82-6$ & $520-7300$ & 323 & 760 & 0.112 & $\mathrm{~N}_{2}$ & Johnson et al. (2010) [519] \\
\hline cis-1,3-Dichloro propene & $10061-01-5$ & $600-6500$ & $278-323$ & 760 & 0.112 & $\mathrm{~N}_{2}$ & Sharpe et al. (2004) [314] \\
\hline Crotonaldehyde & $123-73-9$ & $500-6500$ & $278-323$ & 760 & 0.112 & $\mathrm{~N}_{2}$ & Johnson et al. (2010) [519] \\
\hline Diacetone alcohol & $123-42-2$ & $520-7000$ & $298-323$ & 760 & 0.112 & $\mathrm{~N}_{2}$ & Johnson et al. (2010) [519] \\
\hline Dibromomethane & $74-95-3$ & $500-7100$ & $278-323$ & $700-760$ & $0.112-0.5$ & $\mathrm{~N}_{2}$, air & $\begin{array}{l}\text { Sharpe et al. (2004) [314], } \\
\text { Sihra et al. (2001) [542]* }\end{array}$ \\
\hline Dichloromethane & $75-09-2$ & $600-6500$ & $278-323$ & 760 & 0.112 & $\mathrm{~N}_{2}$ & $\begin{array}{l}\text { Sharpe et al. (2004) [314], } \\
\text { Wallington et al. (2016) } \\
{[540]^{*}}\end{array}$ \\
\hline Dichloromethanol & & $657-2500$ & 295 & 700 & 0.25 & $\mathrm{~N}_{2}$ & $\begin{array}{l}\text { Wallington et al. (2000) } \\
\text { [565] }\end{array}$ \\
\hline Diethylamine & $109-89-7$ & $590-7000$ & $278-323$ & 760 & 0.112 & $\mathrm{~N}_{2}$ & Sharpe et al. (2004) [314] \\
\hline Diiodomethane & $75-11-6$ & $530-7100$ & $298-323$ & 760 & 0.112 & $\mathrm{~N}_{2}$ & Sharpe et al. (2004) [314] \\
\hline Diisopropylamine & $108-18-9$ & $550-6500$ & $278-323$ & 760 & 0.112 & $\mathrm{~N}_{2}$ & Sharpe et al. (2004) [314] \\
\hline Dimethyl disulfide & $624-92-0$ & $600-6500$ & $278-323$ & 760 & 0.112 & $\mathrm{~N}_{2}$ & Sharpe et al. (2004) [314] \\
\hline Dimethyl ether & $115-10-6$ & $600-6500$ & $278-323$ & 760 & 0.112 & $\mathrm{~N}_{2}$ & Sharpe et al. (2004) [314] \\
\hline Dimethyl sulfate & $77-78-1$ & $525-6500$ & $298-323$ & 760 & 0.112 & $\mathrm{~N}_{2}$ & Sharpe et al. (2004) [314] \\
\hline Dimethyl sulfide & $75-18-3$ & $600-6500$ & $278-323$ & 760 & 0.112 & $\mathrm{~N}_{2}$ & Sharpe et al. (2004) [314] \\
\hline Dimethyl sulfoxide & $67-68-5$ & $575-6500$ & $298-323$ & 760 & 0.112 & $\mathrm{~N}_{2}$ & Sharpe et al. (2004) [314] \\
\hline Dimethylamine & $124-40-3$ & $550-6700$ & $278-323$ & 760 & 0.112 & $\mathrm{~N}_{2}$ & Sharpe et al. (2004) [314] \\
\hline D-Limonene & $5989-27-5$ & $520-6500$ & $298-323$ & 760 & 0.112 & $\mathrm{~N}_{2}$ & Johnson et al. (2010) [519] \\
\hline DL-Limonene & $138-86-3$ & $580-6500$ & $298-323$ & 760 & 0.112 & $\mathrm{~N}_{2}$ & Johnson et al. (2010) [519] \\
\hline
\end{tabular}

(continued on next page) 
Table 11 (continued)

\begin{tabular}{|c|c|c|c|c|c|c|c|}
\hline Molecule & CAS & $\begin{array}{l}\text { Wavenumber } \\
\text { range, } \mathrm{cm}^{-1}\end{array}$ & $\mathrm{~T}, \mathrm{~K}$ & P,Torr & Resolution, $\mathrm{cm}^{-1}$ & Broadener & Reference \\
\hline E-C10F18 & $60433-12-7$ & $650-1500$ & 296 & 700 & 0.5 & air & Shine et al. (2005) [566] \\
\hline EDB & $106-93-4$ & $570-6500$ & $278-323$ & 760 & 0.112 & $\mathrm{~N}_{2}$ & Sharpe et al. (2004) [314] \\
\hline Ethyl acetate & $141-78-6$ & $565-6500$ & $278-323$ & 760 & 0.112 & $\mathrm{~N}_{2}$ & Sharpe et al. (2004) [314] \\
\hline Ethyl acrylate & $140-88-5$ & $580-6500$ & $278-323$ & 760 & 0.112 & $\mathrm{~N}_{2}$ & Sharpe et al. (2004) [314] \\
\hline Ethyl alcohol & $64-17-5$ & $600-7500$ & $278-323$ & 760 & 0.112 & $\mathrm{~N}_{2}$ & Sharpe et al. (2004) [314] \\
\hline Ethyl benzoate & $93-89-0$ & $600-6500$ & 323 & 760 & 0.112 & $\mathrm{~N}_{2}$ & Johnson et al. (2010) [519] \\
\hline Ethyl formate & $109-94-4$ & $600-6500$ & $278-323$ & 760 & 0.112 & $\mathrm{~N}_{2}$ & Sharpe et al. (2004) [314] \\
\hline Ethylamine & $75-04-7$ & $600-7000$ & $278-323$ & 760 & 0.112 & $\mathrm{~N}_{2}$ & Sharpe et al. (2004) [314] \\
\hline Ethylenediamine & $107-15-3$ & $550-6500$ & $278-323$ & 760 & 0.112 & $\mathrm{~N}_{2}$ & Sharpe et al. (2004) [314] \\
\hline Ethyliodide & $75-03-6$ & $600-6500$ & $278-323$ & 760 & 0.112 & $\mathrm{~N}_{2}$ & Sharpe et al. (2004) [314] \\
\hline EVE & $109-92-2$ & $570-6500$ & $278-323$ & 760 & 0.112 & $\mathrm{~N}_{2}$ & Sharpe et al. (2004) [314] \\
\hline Fluoroether E-1 & $3330-15-2$ & $450-1450$ & 298 & & 1.0 & & Oyaro et al. (2005) [559] \\
\hline Formic acid, dimer & $14523-98-9$ & $540-7200$ & $278-298$ & 760 & 0.112 & $\mathrm{~N}_{2}$ & Sharpe et al. (2004) [314] \\
\hline Furan & $110-00-9$ & $550-6500$ & $278-323$ & 760 & 0.112 & $\mathrm{~N}_{2}$ & Sharpe et al. (2004) [314] \\
\hline Furfural & $98-01-1$ & $550-6500$ & $298-323$ & 760 & 0.112 & $\mathrm{~N}_{2}$ & Johnson et al. (2010) [519] \\
\hline Furfuryl alcohol & $98-00-0$ & $550-7300$ & $298-323$ & 760 & 0.112 & $\mathrm{~N}_{2}$ & Sharpe et al. (2004) [314] \\
\hline Geraniol & $106-24-1$ & $600-6500$ & 323 & 760 & 0.112 & $\mathrm{~N}_{2}$ & Johnson et al. (2010) [519] \\
\hline Glycolaldehyde & $141-46-8$ & $570-6500$ & $298-332$ & 760 & 0.112 & $\mathrm{~N}_{2}$ & Johnson et al. (2010) [519] \\
\hline Glyoxal & $107-22-2$ & $600-6500$ & $278-323$ & 760 & 0.112 & $\mathrm{~N}_{2}$ & Johnson et al. (2010) [519] \\
\hline Guaiacol & $90-05-1$ & $520-7250$ & $298-323$ & 760 & 0.112 & $\mathrm{~N}_{2}$ & Johnson et al. (2010) [519] \\
\hline Halon-1201 & $1511-62-2$ & $500-1550$ & 296 & 700 & 0.5 & air & Sihra et al. (2001) [542] \\
\hline Halon-1202 & $75-61-6$ & $575-6500$ & $278-323$ & 760 & 0.112 & $\mathrm{~N}_{2}$ & Sharpe et al. (2004) [314] \\
\hline Halon-1211 & $353-59-3$ & $500-6500$ & $278-323$ & $700-760$ & $0.112-0.5$ & $\mathrm{~N}_{2}$, air & $\begin{array}{l}\text { Sharpe et al. (2004) [314], } \\
\text { Sihra et al. (2001) [542]* }\end{array}$ \\
\hline Halon-1301 & $75-63-8$ & $510-6500$ & $278-323$ & $700-760$ & $0.112-0.5$ & $\mathrm{~N}_{2}$, air & $\begin{array}{l}\text { Sharpe et al. (2004) [314], } \\
\text { Sihra et al. (2001) [542]* }\end{array}$ \\
\hline Halon-2311 & $151-67-7$ & $600-1499$ & 298 & 700 & 0.25 & air & $\begin{array}{l}\text { Andersen et al. (2012a) } \\
\text { [567] }\end{array}$ \\
\hline Halon-2402 & $124-73-2$ & $500-6500$ & $278-323$ & $700-760$ & $0.112-0.5$ & $\mathrm{~N}_{2}$, air & $\begin{array}{l}\text { Sharpe et al. (2004) [314], } \\
\text { Sihra et al. (2001) [542]* }\end{array}$ \\
\hline HCFC-121 & $354-14-3$ & $560-6500$ & $278-323$ & 760 & 0.112 & $\mathrm{~N}_{2}$ & Sharpe et al. (2004) [314] \\
\hline HCFC-132a & $354-23-4$ & $520-6500$ & $278-323$ & 760 & 0.112 & $\mathrm{~N}_{2}$ & Sharpe et al. (2004) [314] \\
\hline HCFC-133a & $75-88-7$ & $500-7100$ & $278-323$ & $7-760$ & $0.01-1.0$ & $\mathrm{~N}_{2}$, He & $\begin{array}{l}\text { Sharpe et al. (2004) [314], } \\
\text { McGillen et al. (2015) } \\
\text { [536]*, Etminan et al. } \\
(2014) \text { [530] }\end{array}$ \\
\hline HCFE-235ca2 & $13838-16-9$ & $600-1450$ & 298 & 700 & 0.25 & air & $\begin{array}{l}\text { Andersen et al. (2012a) } \\
\text { [567] }\end{array}$ \\
\hline HCFE-235da2 & $26675-46-7$ & $350-4000$ & $296-298$ & $0-700$ & $0.25-1.0$ & air, self & $\begin{array}{l}\text { Andersen et al. (2010) } \\
\text { [568], Ryan and Nielsen } \\
\text { (2010) [569]*, Sihra et al. } \\
\text { (2001) [542]* }\end{array}$ \\
\hline Heptafluorobutyraldehyde & $375-02-0$ & $600-2500$ & 296 & 700 & 0.5 & air & $\begin{array}{l}\text { Hashikawa et al. (2004) } \\
\text { [562] }\end{array}$ \\
\hline Hexachloro-1,3-butadiene & $87-68-3$ & $530-6500$ & $298-323$ & 760 & 0.112 & $\mathrm{~N}_{2}$ & Sharpe et al. (2004) [314] \\
\hline Hexachlorocyclopentadiene & $77-47-4$ & $575-6500$ & 323 & 760 & 0.112 & $\mathrm{~N}_{2}$ & Sharpe et al. (2004) [314] \\
\hline Hexafluorobenzene & $392-56-3$ & $580-6500$ & $298-323$ & 760 & 0.112 & $\mathrm{~N}_{2}$ & Sharpe et al. (2004) [314] \\
\hline Hexafluoropropene & $116-15-4$ & $100-6500$ & $278-323$ & $740-760$ & $0.112-1.0$ & $\mathrm{~N}_{2}$, air & $\begin{array}{l}\text { Sharpe et al. (2004) [314], } \\
\text { Acerboni et al. (2001) } \\
{[570]^{*}}\end{array}$ \\
\hline Hexyl acetate & $142-92-7$ & $580-6500$ & $298-323$ & 760 & 0.112 & $\mathrm{~N}_{2}$ & Johnson et al. (2010) [519] \\
\hline HFC-1234yf & $754-12-1$ & $700-2000$ & 296 & 700 & 0.25 & air & Nielsen et al. (2007) [571] \\
\hline HFC-1345zfc & $374-27-6$ & 650-1999 & 298 & 700 & 0.25 & air & $\begin{array}{l}\text { Andersen et al. (2012b) } \\
\text { [555] }\end{array}$ \\
\hline HFC-143 & $430-66-0$ & $350-1550$ & 296 & 700 & 0.5 & air & Sihra et al. (2001) [542] \\
\hline HFC-152 & $624-72-6$ & $700-3100$ & 296 & 700 & 0.25 & air & $\begin{array}{l}\text { Wallington et al. (1994) } \\
\text { [572] }\end{array}$ \\
\hline HFC-161 & $353-36-6$ & $350-1600$ & 296 & 700 & 0.5 & air & Sihra et al. (2001) [542] \\
\hline HFC-227ca & $2252-84-8$ & $400-1550$ & 296 & 700 & 0.5 & air & Sihra et al. (2001) [542] \\
\hline HFC-227ea & $431-89-0$ & $400-6500$ & $278-323$ & $700-760$ & $0.112-0.5$ & $\mathrm{~N}_{2}$, air & $\begin{array}{l}\text { Sharpe et al. (2004) [314], } \\
\text { Gohar et al. (2004) [545] }\end{array}$ \\
\hline HFC-23 & $75-46-7$ & $950-1500$ & $187-295$ & $23-763$ & 0.015 & Air & $\begin{array}{l}\text { Harrison JQSRT (2013) } \\
\text { [526], }\end{array}$ \\
\hline HFC-236cb & $677-56-5$ & $300-1550$ & 296 & 700 & 0.5 & air & Sihra et al. (2001) [542] \\
\hline HFC-236fa & $690-39-1$ & $350-1500$ & 296 & 700 & 0.5 & air & Sihra et al. (2001) [542] \\
\hline HFC-245cb & $1814-88-6$ & $400-1550$ & 296 & 700 & 0.5 & air & Sihra et al. (2001) [542] \\
\hline HFC-245fa & $460-73-1$ & $640-1500$ & 296 & 700 & 0.5 & air & Sihra et al. (2001) [542] \\
\hline HFC-272ca & $420-45-1$ & $300-1500$ & 296 & 700 & 0.5 & air & Sihra et al. (2001) [542] \\
\hline HFC-329p & $375-17-7$ & $500-4000$ & 296 & 700 & 0.25 & air & Young et al. (2009b) [544] \\
\hline HFC $-365 \mathrm{mfc}$ & $406-58-6$ & $600-2000$ & 296 & 700 & 0.25 & air & Inoue et al. (2008) [573] \\
\hline HFC-41 & $593-53-3$ & $500-6500$ & $278-323$ & $700-760$ & $0.112-0.5$ & $\mathrm{~N}_{2}$, air & $\begin{array}{l}\text { Sharpe et al. (2004) [314], } \\
\text { Sihra et al. (2001) [542]* }\end{array}$ \\
\hline
\end{tabular}

(continued on next page) 
Table 11 (continued)

\begin{tabular}{|c|c|c|c|c|c|c|c|}
\hline Molecule & CAS & $\begin{array}{l}\text { Wavenumber } \\
\text { range, } \mathrm{cm}^{-1}\end{array}$ & $\mathrm{~T}, \mathrm{~K}$ & P,Torr & Resolution, $\mathrm{cm}^{-1}$ & Broadener & Reference \\
\hline HFE-125 & $3822-68-2$ & $400-1650$ & 296 & 700 & 0.5 & air & Sihra et al. (2001) [542] \\
\hline HFE-143a & $421-14-7$ & $550-1550$ & 296 & 700 & 0.5 & air & Sihra et al. (2001) [542] \\
\hline HFE-216 & $1187-93-5$ & 850-1999 & 296 & 700 & 0.25 & air & Mashino et al. (2000) [574] \\
\hline HFE-227ea & $2356-62-9$ & $400-3200$ & 298 & & 1.0 & & Oyaro et al. (2005) [559] \\
\hline HFE-236ea2 & $57041-67-5$ & $450-3200$ & 298 & 700 & $0.25-1.0$ & air & $\begin{array}{l}\text { Andersen et al. (2010) } \\
{[568]^{*} \text {, Oyaro et al. }} \\
(2005) \text { [559] }\end{array}$ \\
\hline HFE-245fa2 & $1885-48-9$ & $350-3200$ & $296-298$ & 700 & $0.5-1.0$ & air & $\begin{array}{l}\text { Oyaro et al. (2005) [559]*, } \\
\text { Sihra et al. (2001) [542] }\end{array}$ \\
\hline HFE-263fb1 & $460-43-5$ & $450-3300$ & $296-298$ & 700 & $0.25-1.0$ & air & $\begin{array}{l}\text { Osterstrom et al. (2012) } \\
\text { [575], Oyaro et al. (2005) } \\
\text { [559]* }\end{array}$ \\
\hline HFE-263m1 & $690-22-2$ & $450-3200$ & 298 & & 1.0 & & Oyaro et al. (2005) [559] \\
\hline HFE-329me3 & $428454-68-6$ & $600-2000$ & 296 & 700 & 0.25 & air & $\begin{array}{l}\text { Wallington et al. (2004) } \\
\text { [576] }\end{array}$ \\
\hline HFE-338mec 3 & $56860-85-6$ & $500-3500$ & $296-298$ & 700 & $0.25-1.0$ & air & $\begin{array}{l}\text { Wallington et al. (2004) } \\
\text { [576], Oyaro et al. (2005) } \\
\text { [559]* }\end{array}$ \\
\hline HFE-347mcc3 & $375-03-1$ & $700-1400$ & 298 & & 0.03 & & Bravo et al. (2010a) [564] \\
\hline HFE-347mmz1 & $28523-86-6$ & $400-4000$ & 298 & $0-700$ & $0.25-1.0$ & air, self & $\begin{array}{l}\text { Andersen et al. (2010) } \\
\text { [568], Ryan and Nielsen } \\
\text { (2010) [569]* }\end{array}$ \\
\hline HFE-356mff2 & $333-36-8$ & $475-3100$ & $296-298$ & 700 & $0.5-1.0$ & air & $\begin{array}{l}\text { Oyaro et al. (2004) [558]*, } \\
\text { Sihra et al. (2001) [542] }\end{array}$ \\
\hline HFE-356mmz1 & $13171-18-1$ & $450-3200$ & 298 & & 1.0 & & Oyaro et al. (2004) [558] \\
\hline HFE-365mcf3 & $378-16-5$ & $435-3150$ & 298 & & 1.0 & & Oyaro et al. (2004) [558] \\
\hline HFE-449s1 & & $350-1550$ & $296-298$ & $0-700$ & $0.01-0.5$ & air, self & $\begin{array}{l}\text { Sihra et al. (2001) [542]*, } \\
\text { Bravo et al. (2010a) [564] }\end{array}$ \\
\hline HFE-7200 & & $500-1600$ & $296-298$ & $0-700$ & $0.01-0.5$ & air, self & $\begin{array}{l}\text { Sihra et al. (2001) [542]*, } \\
\text { Bravo et al. (2010a) [564] }\end{array}$ \\
\hline HFE-7300 & $132182-92-4$ & $600-2000$ & 298 & & 1.0 & & $\begin{array}{l}\text { Rodriguez et al. (2014) } \\
\text { [539] }\end{array}$ \\
\hline HFE-7500 & 297730-93-9 & $600-2000$ & 298 & & 1.0 & & $\begin{array}{l}\text { Rodriguez et al. (2014) } \\
\text { [539] }\end{array}$ \\
\hline HFIP & & $530-3400$ & $304-362$ & & 0.1 & & Godin et al. (this issue) \\
\hline HFO-1243zf & $677-21-4$ & 500-1999 & $296-298$ & 700 & $0.25-1.0$ & air, He & $\begin{array}{l}\text { Andersen et al. (2012b) } \\
\text { [555], Gonzalez et al. } \\
\text { (2015) [534]* }\end{array}$ \\
\hline (E)-HFC-1234ze & $1645-83-6$ & $650-2000$ & 296 & 700 & 0.25 & air & $\begin{array}{l}\text { Sondergaard et al. (2007) } \\
\text { [577] }\end{array}$ \\
\hline HFO-1438ezy(E) & & 600-1997 & 296 & & 1.0 & $\mathrm{He}$ & $\begin{array}{l}\text { Papadimitriou and } \\
\text { Burkholder. (2016) [537] }\end{array}$ \\
\hline HG-01 & $188690-78-0$ & $25-2999$ & 296 & & 1.0 & air & Myhre et al. (1999) [578] \\
\hline$H G^{\prime}-02$ & $485399-46-0$ & $150-1800$ & 296 & 700 & 0.25 & air & $\begin{array}{l}\text { Andersen et al. (2004) } \\
\text { [557] }\end{array}$ \\
\hline$H G^{\prime}-03$ & $485399-48-2$ & $150-1800$ & 296 & 700 & 0.25 & air & $\begin{array}{l}\text { Andersen et al. (2004) } \\
\text { [557] }\end{array}$ \\
\hline HG-10 & $78522-47-1$ & $25-3000$ & 296 & & 1.0 & air & Myhre et al. (1999) [578] \\
\hline HG-11 & & $400-2000$ & 296 & 700 & $0.25-0.5$ & air & $\begin{array}{l}\text { Wallington et al. (2009) } \\
\text { [579], Sihra et al. (2001) } \\
\text { [542]* }\end{array}$ \\
\hline Hydrazine & $302-01-2$ & $600-7000$ & $298-323$ & 760 & 0.112 & $\mathrm{~N}_{2}$ & Sharpe et al. (2004) [314] \\
\hline i-HFE-7100 & $163702-08-7$ & $300-1650$ & 296 & 700 & 0.5 & air & Sihra et al. (2001) [542] \\
\hline i-HFE-7200 & $163702-06-5$ & $400-1500$ & 296 & 700 & 0.5 & air & Sihra et al. (2001) [542] \\
\hline Isobutane & $75-28-5$ & $600-6500$ & $278-323$ & 760 & 0.112 & $\mathrm{~N}_{2}$ & Sharpe et al. (2004) [314] \\
\hline Isobutene & $115-11-7$ & $600-6500$ & $278-323$ & 760 & 0.112 & $\mathrm{~N}_{2}$ & Sharpe et al. (2004) [314] \\
\hline Isobutyric acid & $79-31-2$ & $530-7250$ & 340 & 760 & 0.112 & $\mathrm{~N}_{2}$ & Johnson et al. (2010) [519] \\
\hline Isocyanic acid & $75-13-8$ & $500-7000$ & 298 & 760 & 0.112 & $\mathrm{~N}_{2}$ & Sharpe et al. (2004) [314] \\
\hline Isopentane & $78-78-4$ & $600-6500$ & $278-323$ & 760 & 0.112 & $\mathrm{~N}_{2}$ & Sharpe et al. (2004) [314] \\
\hline Isopentyl acetate & $123-92-2$ & $550-6500$ & $298-323$ & 760 & 0.112 & $\mathrm{~N}_{2}$ & Johnson et al. (2010) [519] \\
\hline Isophorone & $78-59-1$ & $600-6500$ & $298-323$ & 760 & 0.112 & $\mathrm{~N}_{2}$ & Sharpe et al. (2004) [314] \\
\hline isoprene & $78-79-5$ & $600-6500$ & $278-323$ & 760 & 0.112 & $\mathrm{~N}_{2}$ & Sharpe et al. (2004) [314] \\
\hline Isopropylamine & $75-31-0$ & $600-6800$ & $278-323$ & 760 & 0.112 & $\mathrm{~N}_{2}$ & Sharpe et al. (2004) [314] \\
\hline Isovaleraldehyde & $590-86-3$ & $500-6500$ & $278-323$ & 760 & 0.112 & $\mathrm{~N}_{2}$ & Johnson et al. (2010) [519] \\
\hline Limonene oxide & $1195-92-2$ & $510-6500$ & $298-323$ & 760 & 0.112 & $\mathrm{~N}_{2}$ & Johnson et al. (2010) [519] \\
\hline m-Cresol & $108-39-4$ & $570-6500$ & $298-323$ & 760 & 0.112 & $\mathrm{~N}_{2}$ & Sharpe et al. (2004) [314] \\
\hline Menthol & $89-78-1$ & $580-6500$ & 323 & 760 & 0.112 & $\mathrm{~N}_{2}$ & Johnson et al. (2010) [519] \\
\hline Methyl 2-methyl butyrate & $868-57-5$ & $520-6500$ & $298-323$ & 760 & 0.112 & $\mathrm{~N}_{2}$ & Johnson et al. (2010) [519] \\
\hline Methyl acetoacetate & $105-45-3$ & $520-6500$ & $298-323$ & 760 & 0.112 & $\mathrm{~N}_{2}$ & Johnson et al. (2010) [519] \\
\hline
\end{tabular}

(continued on next page) 
Table 11 (continued)

\begin{tabular}{|c|c|c|c|c|c|c|c|}
\hline Molecule & CAS & $\begin{array}{l}\text { Wavenumber } \\
\text { range, } \mathrm{cm}^{-1}\end{array}$ & $\mathrm{~T}, \mathrm{~K}$ & P,Torr & Resolution, $\mathrm{cm}^{-1}$ & Broadener & Reference \\
\hline Methyl butyl ether & $628-28-4$ & $600-6500$ & $278-323$ & 760 & 0.112 & $\mathrm{~N}_{2}$ & Sharpe et al. (2004) [314] \\
\hline Methyl glyoxal & $78-98-8$ & $550-6500$ & $298-323$ & 760 & 0.112 & $\mathrm{~N}_{2}$ & Johnson et al. (2010) [519] \\
\hline Methyl iodide & $74-88-4$ & $500-6900$ & $278-323$ & 760 & 0.112 & $\mathrm{~N}_{2}$ & Sharpe et al. (2004) [314] \\
\hline Methyl isoamyl ketone & $110-12-3$ & $550-6500$ & $298-323$ & 760 & 0.112 & $\mathrm{~N}_{2}$ & Sharpe et al. (2004) [314] \\
\hline Methyl isothio-cyanate & $556-61-6$ & $600-6500$ & $278-323$ & 760 & 0.112 & $\mathrm{~N}_{2}$ & Sharpe et al. (2004) [314] \\
\hline Methyl mercaptan & $74-93-1$ & $600-6500$ & $278-323$ & 760 & 0.112 & $\mathrm{~N}_{2}$ & Sharpe et al. (2004) [314] \\
\hline Methyl methacrylate & $80-62-6$ & $550-6500$ & $278-323$ & 760 & 0.112 & $\mathrm{~N}_{2}$ & Sharpe et al. (2004) [314] \\
\hline Methyl nitrite & $624-91-9$ & $520-6500$ & $278-323$ & 760 & 0.112 & $\mathrm{~N}_{2}$ & Sharpe et al. (2004) [314] \\
\hline Methyl pivalate & $598-98-1$ & $550-6500$ & $278-323$ & 760 & 0.112 & $\mathrm{~N}_{2}$ & Sharpe et al. (2004) [314] \\
\hline Methyl propionate & $554-12-1$ & $530-6500$ & $278-323$ & 760 & 0.112 & $\mathrm{~N}_{2}$ & Sharpe et al. (2004) [314] \\
\hline Methyl propyl ketone & $107-87-9$ & $520-6500$ & $278-323$ & 760 & 0.112 & $\mathrm{~N}_{2}$ & Johnson et al. (2010) [519] \\
\hline Methyl salicylate & $119-36-8$ & $550-6500$ & $298-323$ & 760 & 0.112 & $\mathrm{~N}_{2}$ & Sharpe et al. (2004) [314] \\
\hline Methyl vinyl ether & $107-25-5$ & $550-6500$ & $278-323$ & 760 & 0.112 & $\mathrm{~N}_{2}$ & Johnson et al. (2010) [519] \\
\hline Methylethyl ketone & $78-93-3$ & $550-6500$ & $278-323$ & 760 & 0.112 & $\mathrm{~N}_{2}$ & Sharpe et al. (2004) [314] \\
\hline Myrcene & $123-35-3$ & $520-6500$ & $298-323$ & 760 & 0.112 & $\mathrm{~N}_{2}$ & Johnson et al. (2010) [519] \\
\hline N,N-Diethylformamide & $617-84-5$ & $520-6500$ & $298-323$ & 760 & 0.112 & $\mathrm{~N}_{2}$ & $\begin{array}{l}\text { Sharpe et al. (2004) [314], } \\
\text { Johnson et al. (2010) } \\
\text { [519] }\end{array}$ \\
\hline $\mathrm{N}, \mathrm{N}$-Diethylaniline & $91-66-7$ & $550-6500$ & $298-323$ & 760 & 0.112 & $\mathrm{~N}_{2}$ & Sharpe et al. (2004) [314] \\
\hline Naphthalene & $91-20-3$ & $600-6500$ & 323 & 760 & 0.112 & $\mathrm{~N}_{2}$ & Sharpe et al. (2004) [314] \\
\hline n-Butane & $106-97-8$ & $600-6500$ & $278-323$ & 760 & 0.112 & $\mathrm{~N}_{2}$ & Sharpe et al. (2004) [314] \\
\hline n-Butylamine & $109-73-9$ & $600-7000$ & $278-323$ & 760 & 0.112 & $\mathrm{~N}_{2}$ & Sharpe et al. (2004) [314] \\
\hline $\mathrm{n}-\mathrm{C}_{3} \mathrm{~F}_{7} \mathrm{OCH}_{3}$ & & $600-2000$ & 295 & 700 & 0.25 & $\mathrm{~N}_{2}$ & $\begin{array}{l}\text { Ninomiya et al. (2000) } \\
\text { [580] }\end{array}$ \\
\hline n-Heptane & $142-82-5$ & $600-6500$ & $278-323$ & 760 & 0.112 & $\mathrm{~N}_{2}$ & Sharpe et al. (2004) [314] \\
\hline n-Hexadecane & $544-76-3$ & $550-6500$ & 323 & 760 & 0.112 & $\mathrm{~N}_{2}$ & Johnson et al. (2010) [519] \\
\hline n-Hexane & $110-54-3$ & $600-6500$ & $278-323$ & 760 & 0.112 & $\mathrm{~N}_{2}$ & Sharpe et al. (2004) [314] \\
\hline n-HFE-7100 & $163702-07-6$ & $300-1520$ & 296 & 700 & 0.5 & air & Sihra et al. (2001) [542] \\
\hline Nitrobenzene & $98-95-3$ & $550-6500$ & $298-323$ & 760 & 0.112 & $\mathrm{~N}_{2}$ & Sharpe et al. (2004) [314] \\
\hline Nitrogen trifluoride & $7783-54-2$ & $400-6500$ & $278-323$ & $700-760$ & $0.112-0.5$ & $\mathrm{~N}_{2}$, air & $\begin{array}{l}\text { Sharpe et al. (2004) [314], } \\
\text { Robson et al. (2006) } \\
{[581]^{*}}\end{array}$ \\
\hline Nitrous acid & $7782-77-6$ & $550-6500$ & 298 & 760 & 0.112 & $\mathrm{~N}_{2}$ & Sharpe et al. (2004) [314] \\
\hline Nonafluoropentanal & $375-53-1$ & $600-2500$ & 296 & 700 & 0.5 & air & $\begin{array}{l}\text { Hashikawa et al. (2004) } \\
\text { [562] }\end{array}$ \\
\hline n-Pentadecane & $629-62-9$ & $570-6500$ & $298-323$ & 760 & 0.112 & $\mathrm{~N}_{2}$ & Johnson et al. (2010) [519] \\
\hline Octane & $111-65-9$ & $600-6500$ & $278-323$ & 760 & 0.112 & $\mathrm{~N}_{2}$ & Sharpe et al. (2004) [314] \\
\hline Octanoic acid & $124-07-2$ & $550-6500$ & $298-323$ & 760 & 0.112 & $\mathrm{~N}_{2}$ & Johnson et al. (2010) [519] \\
\hline o-Toluidine & $95-53-4$ & $550-7300$ & $298-323$ & 760 & 0.112 & $\mathrm{~N}_{2}$ & Sharpe et al. (2004) [314] \\
\hline Pentafluoropropionaldehyde & $422-06-0$ & $600-2500$ & 296 & 700 & 0.5 & air & $\begin{array}{l}\text { Hashikawa et al. (2004) } \\
\text { [562] }\end{array}$ \\
\hline Pentane & $109-66-0$ & $570-6500$ & $278-323$ & 760 & 0.112 & $\mathrm{~N}_{2}$ & Sharpe et al. (2004) [314] \\
\hline $\begin{array}{l}\text { Perfluoro-2-methyl-3- } \\
\text { pentanone }\end{array}$ & $756-13-8$ & $450-2000$ & 298 & & 1.0 & air & D’Anna et al. (2005) [582] \\
\hline Perfluorobut-1-ene & $357-26-6$ & $600-2000$ & 296 & 700 & 0.25 & $\mathrm{~N}_{2}$ & Young et al. (2009a) [583] \\
\hline Perfluorobuta-1,3-diene & $685-63-2$ & $100-2600$ & 293 & 740 & 1.0 & air & Acerboni et al. (2001) [570] \\
\hline Perfluorobutane & $355-25-9$ & $450-6500$ & $278-323$ & $700-760$ & $0.01-0.5$ & $\mathrm{~N}_{2}$, air & $\begin{array}{l}\text { Sharpe et al. (2004) [314], } \\
\text { Bravo et al. (2010b) } \\
{[543]^{*}}\end{array}$ \\
\hline Perfluoropentane & $678-26-2$ & $500-6500$ & $278-323$ & $0-760$ & $0.01-0.112$ & $\mathrm{~N}_{2}$, air, self & $\begin{array}{l}\text { Sharpe et al. (2004) [314], } \\
\text { Bravo et al. (2010b) [543] }\end{array}$ \\
\hline PFC-1114 & $116-14-3$ & $100-2600$ & 293 & 740 & 1.0 & air & Acerboni et al. (2001) [570] \\
\hline PFC-218 & $76-19-7$ & $500-6500$ & $278-323$ & $700-760$ & $0.01-0.5$ & $\mathrm{~N}_{2}$, air & $\begin{array}{l}\text { Sharpe et al. (2004) [314], } \\
\text { Sihra et al. (2001) [542]*, } \\
\text { Bravo et al. (2010b) } \\
{[543]^{*}}\end{array}$ \\
\hline PFC-318 & $115-25-3$ & $550-6500$ & $253-323$ & $0-760$ & $0.03-0.112$ & $\mathrm{~N}_{2, \text { self }}$ & $\begin{array}{l}\text { Highwood and Shine } \\
\text { (2000) [521], Sharpe et } \\
\text { al. (2004) [314] }\end{array}$ \\
\hline PFC-51-14 & $355-42-0$ & $700-1400$ & 297 & & 0.01 & & Bravo et al. (2010b) [543] \\
\hline PFC-71-18 & & $700-1400$ & 297 & & 0.01 & & Bravo et al. (2010b) [543] \\
\hline PFC-91-18 & $306-94-5$ & $0-2000$ & 296 & 700 & 0.5 & air & Shine et al. (2005) [566] \\
\hline PFPMIE & $1309353-34-1$ & $600-1500$ & 296 & 700 & 0.25 & air & Young et al. (2006) [584] \\
\hline PFPO & $422-05-9$ & $490-4000$ & $298-362$ & & 0.1 & & $\begin{array}{l}\text { Sellevag et al. (2007) } \\
\text { [533]*, Godin et al. } \\
\text { (2017) [532] }\end{array}$ \\
\hline PFTBA & $311-89-7$ & $550-2000$ & $298-344$ & & 0.1 & & Godin et al. (2016) [531] \\
\hline Phenol & $108-95-2$ & $550-7300$ & $298-323$ & 760 & 0.112 & $\mathrm{~N}_{2}$ & Johnson et al. (2010) [519] \\
\hline Phosgene & $75-44-5$ & $535-6500$ & $278-323$ & 760 & 0.112 & $\mathrm{~N}_{2}$ & Sharpe et al. (2004) [314] \\
\hline Piperidine & $110-89-4$ & $510-6700$ & $278-323$ & 760 & 0.112 & $\mathrm{~N}_{2}$ & Sharpe et al. (2004) [314] \\
\hline Propionaldehyde & $123-38-6$ & $520-6500$ & $278-323$ & 760 & 0.112 & $\mathrm{~N}_{2}$ & Sharpe et al. (2004) [314] \\
\hline Propylene carbonate & $108-32-7$ & $600-6500$ & $298-323$ & 760 & 0.112 & $\mathrm{~N}_{2}$ & Johnson et al. (2010) [519] \\
\hline Propylenimine & $75-55-8$ & $600-6700$ & $278-323$ & 760 & 0.112 & $\mathrm{~N}_{2}$ & Sharpe et al. (2004) [314] \\
\hline $\mathrm{R} 124 \mathrm{~A}$ & $354-25-6$ & $525-6500$ & $278-323$ & 760 & 0.112 & $\mathrm{~N}_{2}$ & Sharpe et al. (2004) [314] \\
\hline
\end{tabular}


Table 11 (continued)

\begin{tabular}{|c|c|c|c|c|c|c|c|}
\hline Molecule & CAS & $\begin{array}{l}\text { Wavenumber } \\
\text { range, } \mathrm{cm}^{-1}\end{array}$ & $\mathrm{~T}, \mathrm{~K}$ & P,Torr & Resolution, $\mathrm{cm}^{-1}$ & Broadener & Reference \\
\hline R132A & $471-43-2$ & $510-6500$ & $278-323$ & 760 & 0.112 & $\mathrm{~N}_{2}$ & Sharpe et al. (2004) [314] \\
\hline R141 & $430-57-9$ & $550-6500$ & $278-323$ & 760 & 0.112 & $\mathrm{~N}_{2}$ & Sharpe et al. (2004) [314] \\
\hline sec-Amylamine & $625-30-9$ & $540-6900$ & $278-323$ & 760 & 0.112 & $\mathrm{~N}_{2}$ & Sharpe et al. (2004) [314] \\
\hline Styrene (monomer) & $100-42-5$ & $600-6500$ & $298-323$ & 760 & 0.112 & $\mathrm{~N}_{2}$ & Sharpe et al. (2004) [314] \\
\hline Sulfuryl fluoride & $2699-79-8$ & $500-6500$ & $278-323$ & $700-760$ & $0.112-0.25$ & $\mathrm{~N}_{2}$, air & $\begin{array}{l}\text { Sharpe et al. (2004) [314], } \\
\text { Andersen et al. (2009) } \\
{[585]^{*}}\end{array}$ \\
\hline TE-6 & 25291-17-2 & 650-1999 & 298 & 700 & 0.25 & air & $\begin{array}{l}\text { Andersen et al. (2012b) } \\
\text { [555] }\end{array}$ \\
\hline Tetrachloroethylene & $127-18-4$ & $600-6500$ & $278-323$ & 760 & 0.112 & $\mathrm{~N}_{2}$ & Sharpe et al. (2004) [314] \\
\hline trans-1,2-Dichloroethene & $156-60-5$ & $540-6500$ & $278-323$ & 760 & 0.112 & $\mathrm{~N}_{2}$ & Sharpe et al. (2004) [314] \\
\hline trans-1,3-Dichloropropene & $10061-02-6$ & $600-6500$ & $278-323$ & 760 & 0.112 & $\mathrm{~N}_{2}$ & Sharpe et al. (2004) [314] \\
\hline Trichloroethylene & $79-01-6$ & $595-6500$ & $278-323$ & 760 & 0.112 & $\mathrm{~N}_{2}$ & Sharpe et al. (2004) [314] \\
\hline Trichloromethane & $67-66-3$ & $580-7200$ & $295-323$ & $700-760$ & $0.01-0.25$ & $\mathrm{~N}_{2}$, self & $\begin{array}{l}\text { Highwood and Shine } \\
\text { (2000) [521], Sharpe et } \\
\text { al. (2004) [314], } \\
\text { Wallington et al. (2016) } \\
\text { [540]* }\end{array}$ \\
\hline Trichloromethanol & & $657-2500$ & 295 & 700 & 0.25 & $\mathrm{~N}_{2}$ & $\begin{array}{l}\text { Wallington et al. (2000) } \\
\text { [565] }\end{array}$ \\
\hline Triethylamine & $121-44-8$ & $575-7100$ & $278-323$ & 760 & 0.112 & $\mathrm{~N}_{2}$ & Sharpe et al. (2004) [314] \\
\hline Trifluoroacetic acid & $76-05-1$ & $540-7050$ & 298 & 760 & 0.112 & $\mathrm{~N}_{2}$ & Sharpe et al. (2004) [314] \\
\hline Trimethylamine & $75-50-3$ & $600-6500$ & $278-323$ & 760 & 0.112 & $\mathrm{~N}_{2}$ & Sharpe et al. (2004) [314] \\
\hline Valeraldehyde & $110-62-3$ & $600-6500$ & $278-323$ & 760 & 0.112 & $\mathrm{~N}_{2}$ & Johnson et al. (2010) [519] \\
\hline Valeric acid & $109-52-4$ & $520-7100$ & 340 & 760 & 0.112 & $\mathrm{~N}_{2}$ & Johnson et al. (2010) [519] \\
\hline Vinyl chloride & $75-01-4$ & $540-6500$ & $278-323$ & 760 & 0.112 & $\mathrm{~N}_{2}$ & Sharpe et al. (2004) [314] \\
\hline Vinyl fluoride & $75-02-5$ & $500-6500$ & $278-323$ & 760 & 0.112 & $\mathrm{~N}_{2}$ & Sharpe et al. (2004) [314] \\
\hline Vinyl toluene & $100-80-1$ & $550-6500$ & $298-323$ & 760 & 0.112 & $\mathrm{~N}_{2}$ & Johnson et al. (2010) [519] \\
\hline Vinyl trifluoroacetate & $433-28-3$ & $600-2000$ & 298 & & 0.03 & air & $\begin{array}{l}\text { Rodrigues et al. (2016) } \\
\text { [538] }\end{array}$ \\
\hline Z-C10F18 & 60433-11-6 & $650-1500$ & 296 & 700 & 0.5 & air & Shine et al. (2005) [566] \\
\hline
\end{tabular}

*Data from these sources can be found in the alternate folder.

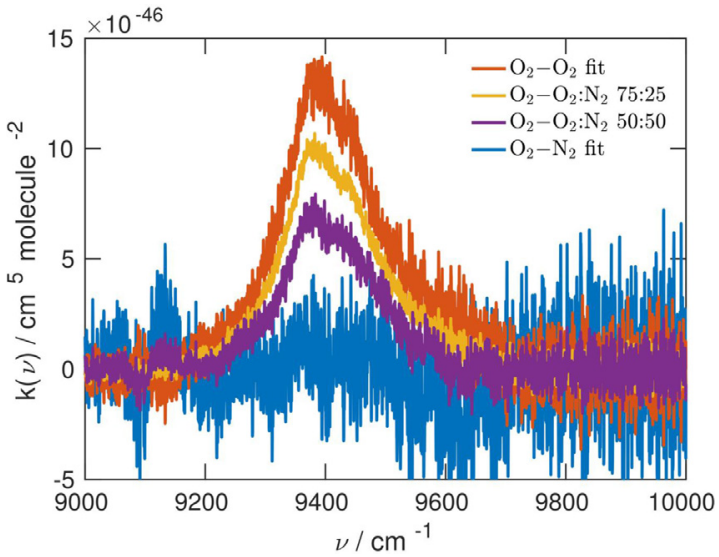

Fig. 28. Raw experimental CIA data for the oxygen $1.06-\mu \mathrm{m}$ band at $T=230 \mathrm{~K}$ for 50:50 and 75:25 $\mathrm{O}_{2}: \mathrm{N}_{2}$ mixtures, and the contributions of $\mathrm{O}_{2}-\mathrm{O}_{2}$ and $\mathrm{O}_{2}-\mathrm{N}_{2}$ determined from these data. For readability in gray scale, the legend is ordered vertically according to the peak intensity.

A plane light wave of wavelength $\lambda$ is attenuated along the propagation $\mathrm{x}$ axis according to

$\mathrm{E}=\mathrm{E}_{0} \exp \left(-2 \pi \mathrm{m}_{\mathrm{imag}} \mathrm{x} / \lambda\right) \exp \left(\mathrm{i} 2 \pi \mathrm{m}_{\text {real }} \mathrm{x} / \lambda-\mathrm{i} 2 \pi \mathrm{ct} / \lambda\right)$,

with time $t$ and the speed of light $c$. The imaginary refractive index $\mathrm{m}_{\text {imag }}$ determines the amount of light absorption in a medium, attenuating the light intensity by $\exp \left(-4 \pi \mathrm{m}_{\mathrm{imag}} \mathrm{x} / \lambda\right)$ along a path of distance $\mathrm{x}$.

New HITRAN2016 indices include refractive indices associated with exoplanet atmospheres. Indices (see Table 12) of materials which condense out at temperatures from $1725 \mathrm{~K}$ (e.g. $\mathrm{SiO}_{2}$ ) to $700 \mathrm{~K}$ (e.g. $\mathrm{ZnS}$ ) include most of the condensates tabulated
Querry ZnS

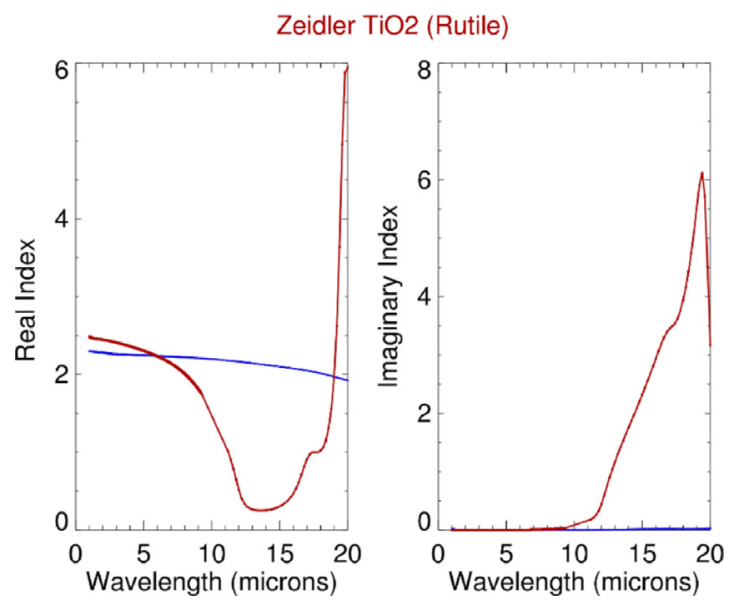

Fig. 29. Comparison of the real and imaginary indices of $\mathrm{ZnS}$ and $\mathrm{TiO}_{2}$ from 1 to $20 \mu \mathrm{m}$. Since particle extinction spectra in the infrared have a wavelength dependence which is similar to the wavelength dependence of the imaginary index, the extinction spectra of $\mathrm{ZnS}$ and $\mathrm{TiO}_{2}$ particles will be very different.

by Wakeford and Sing [634], who calculated transmission spectral properties of clouds for hot-Jupiter exoplanets. Many of the indices have been kindly provided to HITRAN2016 by Harrald Mutschke of the Friedrich Schiller University Jena. Additional indices of materials (e.g. Mg spinels with a variety of impurities) not tabulated in Table 12 can be downloaded from the extensive Jena web site (http://www.astro.uni-jena.de/Laboratory/OCDB/ index.html). An example of the new indices is presented in Fig. 29, which displays the real and imaginary indices of $\mathrm{ZnS}$ and $\mathrm{TiO}_{2}$, two possible exoplanet particle compositions. 
Table 12

Refractive indices included in HITRAN2016.

\begin{tabular}{|c|c|c|}
\hline Compound & Measurement Specifics & Reference \\
\hline Water & $27^{\circ} \mathrm{C}, 10-5000 \mathrm{~cm}^{-1}$ & {$[592]$} \\
\hline Water & $0.67-2.5 \mu \mathrm{m}$ & [593] \\
\hline Ice & $266 \mathrm{~K}, 0.04 \mu \mathrm{m}-2 \mathrm{~m}$ & [594] \\
\hline Ice & $0.67-2.5 \mu \mathrm{m}$ & [593] \\
\hline $\begin{array}{l}\text { Water, ice, sodium chloride, sea } \\
\text { salt, water soluble aerosol, } \\
\text { ammonium sulfate, } \\
\text { carbonaceous aerosol, } \\
\text { volcanic dust, sulfuric acid, } \\
\text { meteoric dust, quartz, } \\
\text { hematite, sand }\end{array}$ & Room temperature, $0.2-40 \mu \mathrm{m}$ & [595] \\
\hline Sulfuric acid $\left(\mathrm{H}_{2} \mathrm{SO}_{4} / \mathrm{H}_{2} \mathrm{O}\right)$ & Room temperature, $25-96 \% \mathrm{H}_{2} \mathrm{SO}_{4}$ & [596] \\
\hline Sulfuric acid $\left(\mathrm{H}_{2} \mathrm{SO}_{4} / \mathrm{H}_{2} \mathrm{O}\right)$ & Room temperature, 75 and $90 \% \mathrm{H}_{2} \mathrm{SO}_{4}$ & [597] \\
\hline Sulfuric acid $\left(\mathrm{H}_{2} \mathrm{SO}_{4} / \mathrm{H}_{2} \mathrm{O}\right)$ & $215 \mathrm{~K}, 499-6996 \mathrm{~cm}^{-1}$ & [598] \\
\hline Sulfuric acid $\left(\mathrm{H}_{2} \mathrm{SO}_{4} / \mathrm{H}_{2} \mathrm{O}\right)$ & $200-300 \mathrm{~K}, 825-4700 \mathrm{~cm}^{-1}$ & [599] \\
\hline Sulfuric acid $\left(\mathrm{H}_{2} \mathrm{SO}_{4} / \mathrm{H}_{2} \mathrm{O}\right)$ & $213-293 \mathrm{~K}, 432-5028 \mathrm{~cm}^{-1}$ & [600] \\
\hline Nitric acid $\left(\mathrm{H}_{2} \mathrm{SO}_{4} / \mathrm{HNO}_{3}\right)$ & Room temperature, $250-2987 \mathrm{~cm}^{-1}$ & [601] \\
\hline Nitric acid $\left(\mathrm{H}_{2} \mathrm{SO}_{4} / \mathrm{HNO}_{3}\right)$ & $220 \mathrm{~K}, 754-4700 \mathrm{~cm}^{-1}$ & [602] \\
\hline Nitric acid $\left(\mathrm{H}_{2} \mathrm{SO}_{4} / \mathrm{HNO}_{3}\right)$ & $213-293 \mathrm{~K}, 432-5028 \mathrm{~cm}^{-1}$ & [600] \\
\hline $\begin{array}{l}\text { Amorphous nitric acid (NAM, } \\
\text { NAD, NAT) }\end{array}$ & $153 \mathrm{~K}, 482-7000 \mathrm{~cm}^{-1}$ & [603] \\
\hline NAM & $179 \mathrm{~K}, 482-6002 \mathrm{~cm}^{-1}$ & [603] \\
\hline NAD & $184 \mathrm{~K}, 482-6981 \mathrm{~cm}^{-1}$ & [603] \\
\hline NAD & $160-190 \mathrm{~K}, 700-4700 \mathrm{~cm}^{-1}$ & [604] \\
\hline$\alpha$ NAT & $181 \mathrm{~K}, 482-6989 \mathrm{~cm}^{-1}$ & [603] \\
\hline$\beta$ NAT & $196 \mathrm{~K}, 482-6364 \mathrm{~cm}^{-1}$ & [603] \\
\hline NAT & $160 \mathrm{~K}, 711-4004 \mathrm{~cm}^{-1}$ & [605] \\
\hline Burning vegetation & $525-5000 \mathrm{~cm}^{-1}$ & [606] \\
\hline Burning vegetation & $0.35-1.5 \mu \mathrm{m}$ & [607] \\
\hline Carbon flame & $0.4-0.7 \mu \mathrm{m}, 25-600{ }^{\circ} \mathrm{C}$ & [608] \\
\hline Flame soot & $0.2-38 \mu \mathrm{m}$ & [609] \\
\hline Diesel soot & $0.45-10 \mu \mathrm{m}$ & [610] \\
\hline Brown carbon & $0.2-1.2 \mu \mathrm{m}$ & [611] \\
\hline $\begin{array}{l}\text { Organic acids (Oxalic, malonic, } \\
\text { succinic, pinonic, pyruvic, } \\
\text { phthalic) }\end{array}$ & $0.25-1.1 \mu \mathrm{m}$ & [612] \\
\hline Organic haze & $0.525 \mathrm{~nm}$ & [613] \\
\hline SOA (proxy) & $0.525 \mathrm{~nm}$ & [614] \\
\hline $\begin{array}{l}\text { Minerals (clay, illite, kaolin, } \\
\text { montmorillonite) }\end{array}$ & $2.5-200 \mu \mathrm{m}$ & {$[610]$} \\
\hline $\begin{array}{l}\text { Minerals (granite, } \\
\text { montmorillonite) }\end{array}$ & $5-40 \mu \mathrm{m}$ & [615] \\
\hline Saharan dust & $0.30-0.95 \mu \mathrm{m}$ & [616] \\
\hline Saharan dust & $0.35-0.65 \mu \mathrm{m}$ & [617] \\
\hline Volcanic ash & $0.45-25 \mu \mathrm{m}$ & [618] \\
\hline $\mathrm{SiO}_{2}$ (amorphous) & $6.6-487 \mathrm{~m}, 10-300 \mathrm{~K}$ & [619] \\
\hline $\mathrm{SiO}_{2}$ (crystalline) & $6.25 \mu \mathrm{m}-10 \mathrm{~mm}, 300-928 \mathrm{~K}$ & [620] \\
\hline $\mathrm{Al}_{2} \mathrm{O}_{3}$ & $7.8-200 \mu \mathrm{m}$ & [621] \\
\hline $\mathrm{FeO}$ & $0.2-500 \mu \mathrm{m}$ & [622] \\
\hline $\mathrm{CaTiO}_{3}$ (Perovskite) & $2.0-500 \mu \mathrm{m}$ & [623] \\
\hline $\mathrm{Fe}_{2} \mathrm{O}_{3}$ & $0.1-1000 \mu \mathrm{m}$ & [624] \\
\hline $\mathrm{Fe}_{2} \mathrm{SiO}_{4}$ (Fayalite) & $0.4-10 \mu \mathrm{m}$ & [625] \\
\hline $\mathrm{Fe}_{2} \mathrm{SiO}_{4}$ (Fayalite) & $2-10 \mathrm{~mm}$ & [626] \\
\hline $\mathrm{MgAl}_{2} \mathrm{O}_{4}$ (annealed) & $1.6-6825 \mu \mathrm{m}$ & [627] \\
\hline $\mathrm{MgAl}_{2} \mathrm{O}_{4}$ (natural) & $2.0 \mu \mathrm{m}-10 \mathrm{~mm}$ & [627] \\
\hline $\mathrm{Mg}_{2} \mathrm{SiO}_{4}$ & $0.19-948 \mu \mathrm{m}$ & [628] \\
\hline $\mathrm{MgSiO}_{3}$ & $0.2-500 \mu \mathrm{m}$ & [628] \\
\hline $\mathrm{TiO}_{2}$ (Rutile) & $0.47-36.2 \mu \mathrm{m}$ & [629] \\
\hline $\mathrm{TiO}_{2}$ (Anatase) & $2.0-5843 \mu \mathrm{m}$ & [629] \\
\hline $\mathrm{TiO}_{2}$ (Brookite) & $2.0-5843 \mu \mathrm{m}$ & [630] \\
\hline $\mathrm{KCl}$ & $0.22-166 \mu \mathrm{m}$ & [630] \\
\hline $\mathrm{ZnS}$ & $0.22-166 \mu \mathrm{m}$ & [630] \\
\hline Titan Tholins & $0.02-920 \mu \mathrm{m}$ & [631] \\
\hline Titan aerosol & $0.2-1 \mu \mathrm{m}$ & {$[632]$} \\
\hline
\end{tabular}

HITRAN2016 extends the HITRAN-RI program [635] that resides on the HITRAN website by including the exoplanet indices. HITRAN-RI is written in the IDL (Interactive Design Language) and Fortran 90 programming languages, and applies the BohrenHuffman [633] Mie code. The user specifies in a user-edited ASCII file the particle composition (refractive index), wavelength range, and log-normal particle size distribution. The program then calculates extinction, scattering, absorption, single scattering albedo, asymmetry, and backscattering spectra of the cloud or aerosol particle. Provision is provided to generate output ASCII files of the inputs and spectra. The IDL version of HITRAN-RI provides output postscript files. Other special program features allow the user to compare two sets of refractive indices, and to calculate spectra for multiple-component aerosols. As an instructional aid, test cases can be run. PDF versions of the original reference papers are contained in a subdirectory, while the refractive indices are stored 
in subdirectories in ASCII and NetCDF formats. The user can use the ASCII files to quickly look up the real and imaginary indices at a specific wavelength, while the HITRAN-RI program accesses the netCDF files. HITRAN-RI allows the user to read in user-specified refractive indices. The source code of HITRAN-RI can also be modified and added to by the user.

\section{Global data and software}

\subsection{Database structure and interface (www.hitran.org)}

Since June 2015, users have been able to access the HITRAN line-by-line data not only by downloading them in the traditional ASCII 160-character (.par) format from the ftp site, but also through the easy-to-use interface HITRANonline, available at www.hitran.org. Cross sections can also be accessed through this dynamic interface, while CIA and aerosols sections of the database are also accessible, though so far only as static files. This new tool (described in Hill et al. [2]) allows custom filtering and selection of molecules and their isotopologues in the wavenumber region of interest. The default output format is still .par, but users are given an opportunity to create their own, customized output formats, including the HDF5 format widely used by NASA scientists. The flexibility of the formats is achieved due to the fact that the database is cast into a relational format [236]: it is now a compilation of tables of parameters and metadata that are interconnected and searchable using Structured Query Language (SQL) (see Refs. [2] and [236] for details). This new flexible database format offers many advantages over the static fixed-width ASCII files. Indeed, we have now introduced non-Voigt line shapes [6], broadening parameters by perturbing gases other than air or self [4], and other parameters that are important for accurate atmospheric retrievals. Other advantages of the new structure include allowing more significant figures (for instance, important for line positions of the MW transitions) and checks on data integrity. Further advantages include immediate access to the references for every parameter (including the automatic generation of bibliography in both HTML and BibTeX format), generation of interactive plots of retrieved line-by-line and cross-section data (with full features such as zoom, etc.), and more-detailed filtering (such as by individual vibrational bands), etc.

HITRANonline has received a warm welcome from the community, with over 6200 researchers registered with it (as of mid-June 2017). The rate of new users signing up on www.hitran.org has not dropped below 50 per week. Extensive documentation regarding the definition and use of HITRAN parameters is now directly accessible on the website.

There are a few new features that have been introduced to the HITRANonline interface since its original release. First, in order to make the selection of several dozen new special parameters less cumbersome, we "bundled" the parameters associated with selected line profiles. In the example shown in Fig. 30 one can see how the Hartmann-Tran profile can be selected.

\subsection{HITRAN application programming interface (HAPI)}

The HITRAN Application Programming Interface (HAPI) is a free open source Python module (library) which provides a set of tools for working with structured spectroscopic data from different sources. It is described in detail in Ref. [5]. Here we give a brief overview of its features.

Originally created as an extension of the HITRANonline web interface [2] to allow users sophisticated calculations and manipulations with the HITRAN data on their computers, HAPI can handle user-supplied custom data in a flexible way and fully sup- ports the HITRANonline data scheme and formats of the HITRAN [1] database.

The principal aim of HAPI is facilitating physically-sound interpretation of observations and more realistic models for a wide variety of applications such as astrophysics, planetary science, climate simulations, remote sensing, theoretical spectroscopy, and data mining. Having such a tool is important in particular to prevent possible errors in radiative-transfer calculations caused by misuse of spectroscopic tools and databases (see example discussed in Ref. [636] for instance).

The HAPI package can be obtained via the HITRANonline website (http://hitran.org/hapi) or from the Zenodo community (http: //zenodo.org/communities/hapi/about/). The current version (1.1) is distributed under a permissive open source license (MIT). Additional links can be found on the official HITRAN site.

HAPI consists of two major parts. First, it has a simple built-in database management system written in pure Python, which gives a capability of data processing and filtering. Second, HAPI incorporates a set of tools for spectra simulation accounting for the temperature, pressure, optical path length, and instrument properties. In more detail, the HAPI features are as follows:

- Support for standard HITRAN parameters given in "160character" or ".par" format which is described in Ref. [3],

- Support for the newly added types of parameters such as foreign broadenings and shifts, speed dependencies for non-Voigt profiles, line mixing coefficients, etc.

- Communication with the HITRANonline web interface using the REST HTTP protocol in order to get the most up-to-date HITRAN data,

- Python implementations for line profiles including the partially correlated quadratic speed-dependent hard collision model (Hartmann-Tran profile $[7,8,13,14]$ ),

- Python implementation of the Total Internal Partition Sums (based on TIPS-2011 [112] and incorporating a large update outlined in this paper),

- High-resolution single-layer spectra simulation accounting for pressure, temperature, optical path length and instrument function. In the simplest case, the simulation is performed in a single step using a functional approach. See Fig. 25 where HAPI was used to generate cross-sections of ethylene to compare with experimental data.

- Capability of modeling the absorption cross sections broadened by a mixture of gases of astrophysical and planetary interests. The parameters for the foreign broadenings and shift are gradually being added to the HITRANonline system (see outline in Refs. [4] [5]). Fig. 31 shows an example of how one can generate cross-sections of acetylene broadened by different planetary-relevant proportions of hydrogen and helium (see Section 2.26 for details).

- Flexibility in the scheme of the absorption cross-section calculation. Each part of the scheme given in Ref. [5] can be customized by user.

- Capability of extending the HAPI functionality by adding custom line shapes, partition sums, instrumental functions, environment dependences, and radiative-transfer code.

- Compatibility with a large set of third-party libraries in Python, $\mathrm{C} / \mathrm{C}++$, Java etc...

In the near future, in order to improve the simulation of the absorption cross sections, it is planned to include the full relaxation matrix calculation for line mixing for a number of molecules of atmospheric importance $[117,238,239]$. Inclusion of the line mixing in the calculation is expected to enable sub-percent accuracy (provided other parameters are known very accurately) for bands of atmospheric gases to better satisfy highly-precise ground and satellite measurements. 


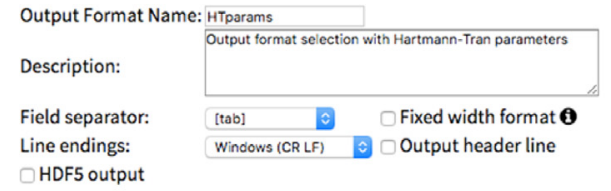

\begin{tabular}{|c|c|c|c|}
\hline \multicolumn{4}{|c|}{ New Output Format } \\
\hline Parameter & Units & Err & Ref \\
\hline \multicolumn{4}{|l|}{ × $(3$ Isotopologue ID } \\
\hline \multicolumn{4}{|l|}{ x (3) Molecule ID } \\
\hline × $\mathbf{\theta} v$ & $\mathrm{~cm}^{-1}$ & 0 & 0 \\
\hline x $6 s$ & $\mathrm{~cm}^{-1} /\left(\operatorname{molec} \cdot \mathrm{cm}^{-2}\right)$ & 0 & 0 \\
\hline \multicolumn{4}{|l|}{ × } \\
\hline x (3) $E^{\prime \prime}$ & $\mathrm{cm}^{-1}$ & & \\
\hline
\end{tabular}

\begin{tabular}{|c|c|c|c|}
\hline \multicolumn{4}{|c|}{ Hartmann-Tran Profile parameters } \\
\hline \multirow[t]{8}{*}{ self } & \multicolumn{3}{|l|}{ air } \\
\hline & $50 \mathrm{~K}$ & $\nabla 150 \mathrm{~K}$ & च296 K \\
\hline & $Y_{H T \_2 \_s e l f}(50)$ & $\delta_{H T \_2 \_s e l f}(150)$ & $\delta_{H T \_0 \_s e l f}(296)$ \\
\hline & $n_{\text {HT_O_self }}(50)$ & $Y_{H T}$ _0_self $(150)$ & $\delta_{H T}^{\prime}{ }_{\text {HT_self }}(296)$ \\
\hline & $Y_{\text {HT_0_self }}(50)$ & $Y_{H T \_2 \text { self }}(150)$ & $V_{H T}$ 0_self $(296)$ \\
\hline & $\delta_{\text {HT_2_self }}(50)$ & $n_{H T \_0 \_s e l f}(150)$ & $n_{H T \_0 \_s e l f}(296)$ \\
\hline & $\delta_{\mathrm{HT}}^{\prime}$ 0_self $(50)$ & $\delta_{H T \_0 \_s e l f}(150)$ & $\delta_{H T \_2 \_s e l f}(296)$ \\
\hline & $\delta_{\mathrm{HT} \_0 \_ \text {self }}(50)$ & $\delta_{H T \_0 \_s e l f}^{\prime}(150)$ & $V_{H T}$ _2_self $(296)$ \\
\hline \multicolumn{4}{|c|}{$700 \mathrm{~K}$} \\
\hline \multicolumn{4}{|c|}{$\delta_{H T \_0 \_s e l f}(700)$} \\
\hline \multicolumn{4}{|c|}{$Y_{H T}{ }_{\text {2_self }}(700)$} \\
\hline \multicolumn{4}{|c|}{ Common Parameters } \\
\hline \multicolumn{4}{|c|}{$n_{\mathrm{HT} \_0 \_s e l f}(700)$} \\
\hline \multicolumn{4}{|c|}{$\delta_{H T \_0 \_s e l f}^{\prime}(700)$} \\
\hline \multicolumn{4}{|c|}{$\delta_{H T \_2 \_s e l f}(700)$} \\
\hline Done & & & \\
\hline
\end{tabular}

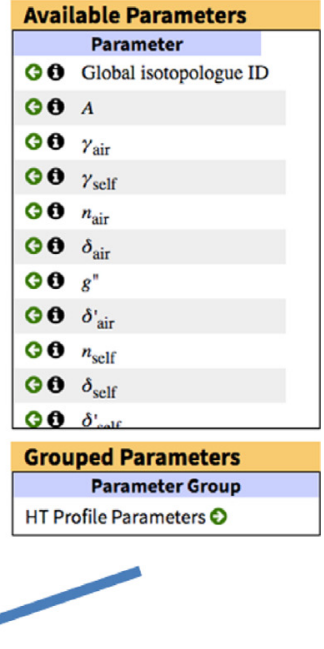

Popup window

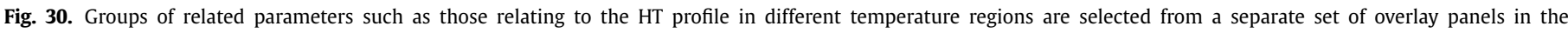

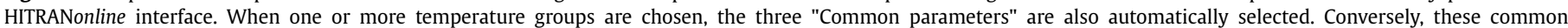

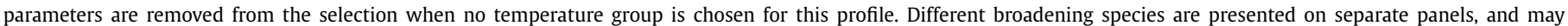
be selected from the labeled tabs at the top of the overlay.

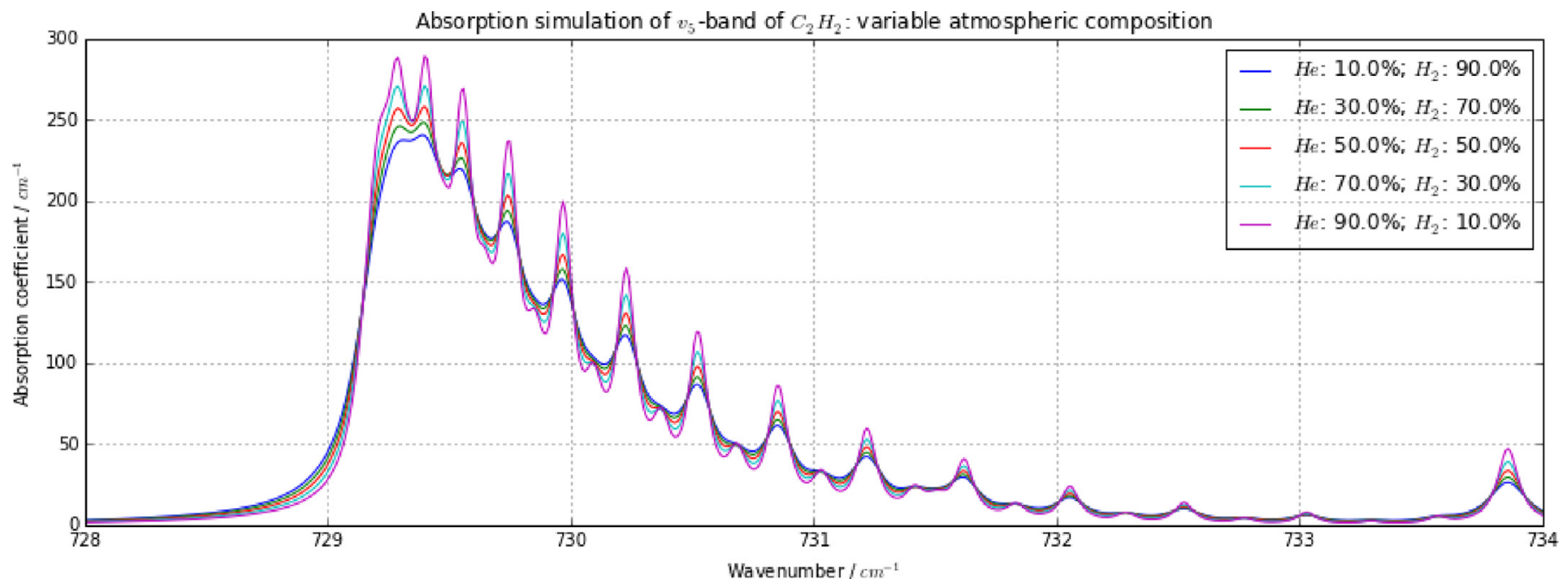

Fig. 31. Cross-sections of acetylene in different environments of hydrogen and helium, generated with HAPI.

At the current time HAPI requires the user to be familiar with Python, although most of the functions are prewritten and with the help of the manual one does not need to have advanced knowledge of that computer language. In order to extend the range of users, we plan to create a flexible graphical user interface (GUI).
This interface will give the user a possibility to simulate, plot and compare line-by-line and raw spectral data with a flexible control of scale, units, and computational parameters (just as is possible in the current Python version of HAPI). The new software will support Windows, Linux and MacOS. 
Table 13

List of isotopologues whose partition sums have been updated for this edition of HITRAN. Note that there a few rare isotopologues here that are not in HITRAN.

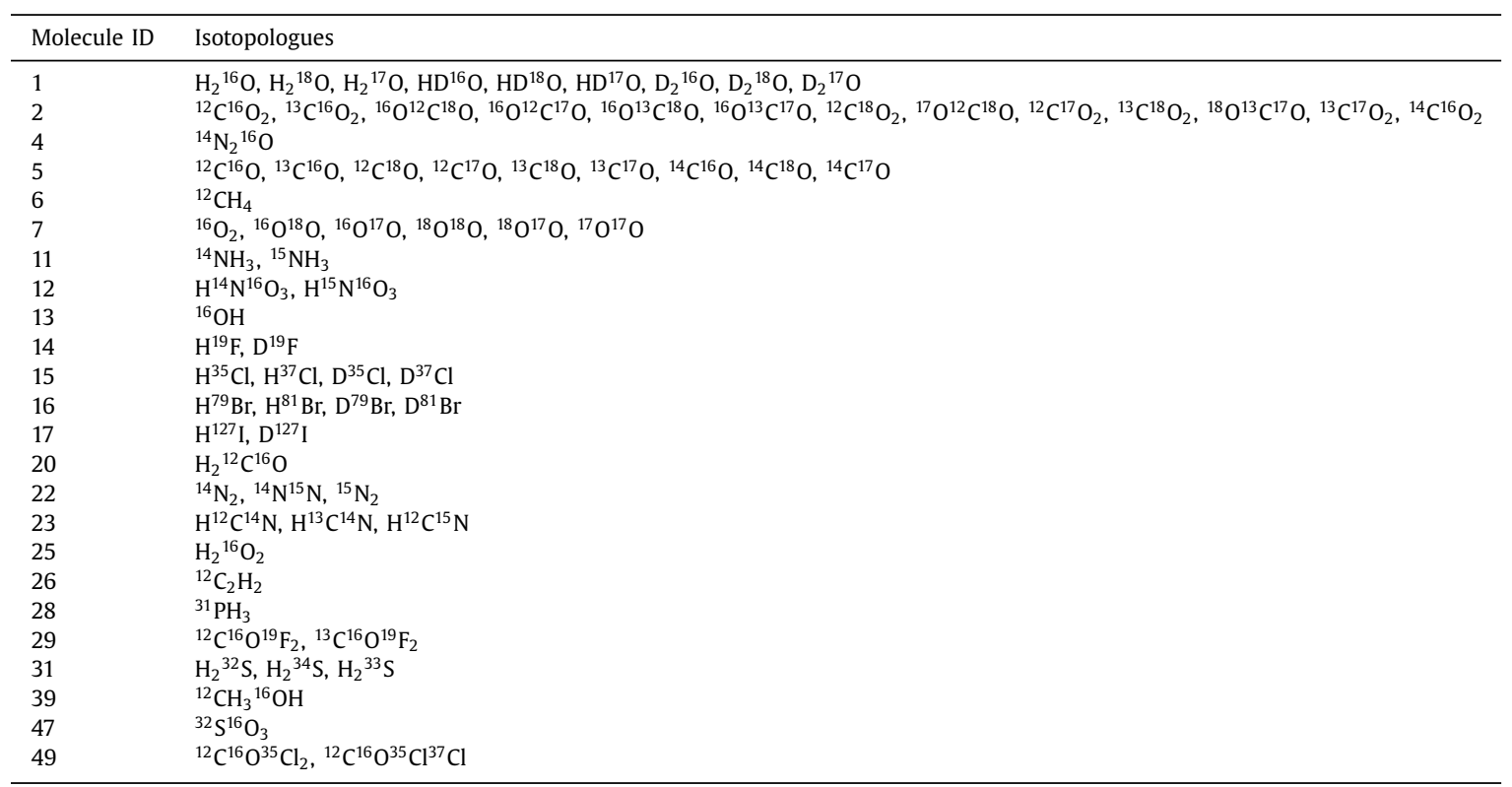

\subsection{Total internal partition sums (TIPS)}

Total internal partition sums (TIPS) were determined for all molecules and isotopologues in HITRAN2016 and for a number of isotopologues of molecules of interest in planetary atmospheres. The current TIPS data are calculated in one-degree steps from $1 \mathrm{~K}$ to $T_{\max }$, where $T_{\max }$ is determined separately for each isotopologue based on convergence of the partition sums. The TIPS are comprised of updated calculations and values from previous studies of TIPS [112,637-639]. The updates can be grouped into several categories: improvements in ab initio calculations that allow direct sums over energy levels, improved $Q_{\text {vib }}$ values using the anharmonic approximation [640], and calculations for new molecule/isotopologues in the HITRAN database. The updates that were made for isotopologues in this edition of HITRAN are listed in Table 13. The updates are detailed in an accompanying paper [641] in this HITRAN2016 special issue. Note that an error was found in Tables 1 and 2 of Ref. [641] where the values corresponding to partition sums of the ${ }^{16} \mathrm{O}^{17} \mathrm{O}$ isotopologue were a factor of six too large. This has been fixed in the official release of HITRAN2016.

The TIPS are made available to the scientific community in the form of tables and codes that rapidly recall the TIPS for any molecule, isotopologue, and temperature. There are several codes that are distributed with this edition of HITRAN: FORTRAN codes TIPS_2017.for and BD_TIPS_2017.for and a python code TIPS_2017.py with associated dictionaries. TIPS_2017.for is a standalone code that queries the user for a molecule, isotopologue, and temperature and returns Q(T). BD_TIPS_2017.for is a subroutine application that users can insert into their codes to return $Q(T)$ for a molecule/isotopologue/temperature selection. The python code uses python dictionaries to recall $\mathrm{Q}(\mathrm{T})$. The dictionaries are labeled by the molecule number and local ID number, see the TIPS article [641] for details. Details are given to develop custom python algorithms for particular molecule/isotopologue combinations. The TIPS python package was incorporated into the HITRAN Application Programming Interface (HAPI) [5] in order to seamlessly integrate into the cross-section generating process. The tables and codes are available at faculty.uml.edu/Robert_Gamache and www.hitran.org.

\section{Conclusions}

The improvements, expansions and new structure in the new HITRAN database release have been elaborated upon. For the lineby-line data, improved line position, intensity, and line-shape parameters for many of the previously existing molecules and isotopologues have been introduced. Many new lines/bands have been added for different molecules and their isotopologues allowing for a more complete database (for atmospheric applications) and for the expansion of the spectral coverage of the existing line lists. Additionally, HITRAN continues evolving in terms of structure and scope. A new relational database structure has been established [2] that enabled significant expansion of the amount of important spectroscopic parameters provided in the database. Literally dozens of new parameters have been introduced that allow accommodation of non-Voigt line shapes [6]; flexible representation of broadening parameters and their temperature dependencies; broadening and shift of spectral lines due to pressure of gases dominant in planetary atmospheres [4] (namely, $\mathrm{H}_{2}$, $\mathrm{He}$ and $\mathrm{CO}_{2}$ ). This extensive expansion of the database would have been very impractical in the old fixed-length ASCII format of previous HITRAN editions. An interface on the internet has also been established (www.hitran.org) that provides the diverse group of HITRAN users with much power to filter, extract, plot, and query the database. Even more power has been given to the user through the introduction of the HITRAN Application Programming Interface (HAPI) [5]. HAPI is currently a set of Python libraries which not only allows users to download data from the database but also to carry out: 1) single-layer absorption calculations at different thermodynamic conditions, accounting for an instrument function, new line shape parametrizations, perturbing gas or gases, etc, 2) extended functionality provided by custom functions, line lists and partition sums.

Several new molecules and isotopologues have been added to the line-by-line portion of the compilation, which improve modeling of the absorption of the terrestrial atmosphere and beyond.

One of the main highlights of the new edition is the monumental expansion of the cross-sectional part of the database which increased (in terms of amount of gases) about six-fold and now fea- 
tures over 300 species. These cross-sections can also be accessible through www.hitran.org where they are divided into categories for easy browsing.

Aerosols and collision induced absorption datasets have also been revised and extended.

The compilation is free and can be accessed through www. hitran.org. We recommend citing this article when using the HITRAN2016 data. If an update to a certain molecule is announced on the website and is being used, then we encourage users to cite when they downloaded the data.

\section{Future work}

While HITRAN continues to be an international standard for reference spectroscopy for atmospheric and planetary gases, there is still substantial room for improvement. In many subsections throughout the paper we mention the avenues for improvements for individual molecules, but they are really just the tip of the iceberg.

The studies of the terrestrial atmosphere require further improvements to the existing parameters and what is more challenging: population of the dataset of the non-Voigt line-shape parameters. As was discussed above, the amount of measurements and calculations of the parameters obtained using the recommended HT profile are scarce and it will take time before a majority of HITRAN lines will have these parameters provided. Also, we remind experimentalists and theoreticians to take extreme care when fitting to these parameters to avoid correlations.

The line mixing for many molecules is now available in HITRAN through diagonal (Rozenkranz) parametrization. Other parametrizations require provision of additional software (just as it is done in this edition in the case of carbon dioxide) that may have to be molecule or even band-specific. HAPI offers an excellent avenue for addition of this extra functionality.

At the moment only seven HITRAN gases have pressure broadening (and their temperature dependence) and pressure shift of lines by $\mathrm{H}_{2}$, He and $\mathrm{CO}_{2}$. We would like to extend this to all gases important for planetary research. Also, in order to aid modeling of the terrestrial atmosphere, we will add parameters associated with broadening of spectral lines by water vapor (which is sufficiently abundant to make an impact on retrievals in the tropics, for instance).

With an exception of a few diatomic molecules (namely NO, $\mathrm{OH}, \mathrm{HF}, \mathrm{HCl}, \mathrm{HBr}, \mathrm{HI}$ and $\mathrm{H}_{2}$ ) HITRAN data are limited to temperatures encountered in the Earth atmosphere and will be deficient when modeling high-temperature environments including stellar and some planetary atmospheres. The users are encouraged to employ the HITEMP database for these applications [278]. However, there are currently only five gases that are provided in $\operatorname{HITEMP}\left(\mathrm{H}_{2} \mathrm{O}, \mathrm{CO}_{2}, \mathrm{CO}, \mathrm{NO}, \mathrm{OH}\right)$ and a major update of the HITEMP database will be released in the near future. This update will include improvement of the spectroscopy of the existing HITEMP gases but also will introduce additional molecules.

While a major update is featured here for the cross-sectional part of the database, we note that it is still far from complete in terms of gases. Moreover, all of the current updates are in the IR part of the spectrum and another substantial update is needed for the UV cross-sections.

A massive revision and expansion of the CIA data is planned for the near future.

Structure and the tools provided with the database are also a very important part of the HITRAN database. We plan further improvements to the documentation for the HITRANonline interface and the development of video tutorials. Finally, development of an interactive interface is planned for HAPI, for which video tutorials will also be created.

\section{Acknowledgments}

The management and development of the HITRAN molecular database has been supported by NASA AURA program grant NNX14AI55G and NASA Planetary Atmosphere grant NNX13AI59G, and NASA PDART grant NNX16AG51G. Portions of the research described in this paper were performed at the Jet Propulsion Laboratory, California Institute of Technology, under contract with the National Aeronautics and Space Administration, Government sponsorship acknowledged.

We gratefully acknowledge the many researchers who provided data, experimental and/or theoretical: Abdulsamee Alkadrou, Lorenzo Barrett, Emma Barton, Chris Benner, Linda Brown, Jeanna Buldyreva, John Coxon, Phillip Coles, Ludovic Daumont, Jolanta Domyslawska, Andre Fayt, Photos Hadjigeorgiou, Shuiming $\mathrm{Hu}$, Ekaterina Karlovets, Samir Kassi, Julien Lamouroux, Daniel Lisak, Lorenzo Lodi, Gang Li, Anwen Liu, Didier Mondelain, Tatyana Petrova, Laurence Régalia, Candice Renaud, Cyril Richard, Alexander M. Solodov, Alexander A. Solodov, Mikhail Tretyakov, Daniel Underwood, Sergey Yurchenko, Nikolai Zobov.

Those who provided independent validations are also acknowledged: Matthew Alvorado, Joshua Baraban, Chris Boone, Athena Coustenis, Jose Fonfria, Erik Kerstel, Xiong Liu, Emile Medvedev, Jana Mendrok, Eli Mlawer, Johannes Orphal, Fabiano Oyafuso, Vivienne Payne, Steven Ross, Kang Sun, Geronimo Villanueva.

\section{References}

[1] Rothman LS, Gordon IE, Babikov Y, Barbe A, Chris Benner D, Bernath PF, et al The HITRAN2012 molecular spectroscopic database. J Quant Spectrosc Radiat Transf 2013;130:4-50. doi:10.1016/j.jqsrt.2013.07.002.

[2] Hill C, Gordon IE, Kochanov RV, Barrett L, Wilzewski JS, Rothman LS. HITRANonline: An online interface and the flexible representation of spectroscopic data in the HITRAN database. J Quant Spectrosc Radiat Transf 2016;177:4-14. doi:10.1016/j.jqsit.2015.12.012.

[3] Rothman LS, Jacquemart D, Barbe A, Chris Benner D, Birk M, Brown LR, et al. The HITRAN 2004 molecular spectroscopic database. J Quant Spectrosc Radiat Transf 2005;96:139-204. doi:10.1016/j.jqsrt.2004.10.008.

[4] Wilzewski JS, Gordon IE, Kochanov RV, Hill C, Rothman LS. $\mathrm{H}_{2}, \mathrm{He}$, and $\mathrm{CO}_{2}$ line-broadening coefficients, pressure shifts and temperature-dependence exponents for the HITRAN database. Part 1: $\mathrm{SO}_{2}, \mathrm{NH}_{3}, \mathrm{HF}, \mathrm{HCl}, \mathrm{OCS}$ and $\mathrm{C}_{2} \mathrm{H}_{2}$. J Quant Spectrosc Radiat Transf 2016;168:193-206. doi:10.1016/j.jqsrt.2015.09. 003

[5] Kochanov RV, Gordon IE, Rothman LS, Wcisło P, Hill C, Wilzewski JS. HITRAN application programming interface (HAPI): a comprehensive approach to working with spectroscopic data. J Quant Spectrosc Radiat Transf 2016;177:15-30. doi:10.1016/j.jqsrt.2016.03.005

[6] Wcisło P, Gordon IE, Tran H, Tan Y, Hu S-M, Campargue A, et al. The implementation of non-Voigt line profiles in the HITRAN database: $\mathrm{H}_{2}$ case study. J Quant Spectrosc Radiat Transf 2016;177:75-91. doi:10.1016/j.jqsrt.2016.01. 024

[7] Tran H, Ngo NH, Hartmann J-M. Efficient computation of some speeddependent isolated line profiles. J Quant Spectrosc Radiat Transf 2013;129:199-203. doi:10.1016/j.jqsrt.2013.06.015.

[8] Tran H, Ngo NH, Hartmann J-M. Erratum to "Efficient computation of some speed-dependent isolated line profiles" [J. Quant. Spectrosc. Radiat. Transfer 129 (2013) 199-203]. J Quant Spectrosc Radiat Transf 2014;134:104. doi:10. 1016/j.jqsrt.2013.10.015.

[9] Boone CD, Walker KA, Bernath PF. Speed-dependent Voigt profile for water vapor in infrared remote sensing applications. J Quant Spectrosc Radiat Transf 2007;105:525-32. doi:10.1016/j.jqsrt.2006.11.015.

[10] Miller CE, Brown LR, Toth RA, Benner DC, Devi VM. Spectroscopic challenges for high accuracy retrievals of atmospheric $\mathrm{CO}_{2}$ and the Orbiting Carbon Observatory (OCO) experiment. Comptes Rendus Phys 2005:6:876-87. doi:10.1016/j.crhy.2005.09.005

[11] Galatry L. Simultaneous effect of doppler and foreign gas broadening on spectral lines. Phys Rev 1961;122:1218-23. doi:10.1103/PhysRev.122.1218.

[12] Pine AS. Asymmetries and correlations in speed-dependent Dicke-narrowed line shapes of argon-broadened HF. J Quant Spectrosc Radiat Transf 1999;62:397-423. doi:10.1016/S0022-4073(98)00112-5.

[13] Tennyson J, Bernath PF, Campargue A, Császár AG, Daumont L, Gamache RR, et al. Recommended isolated-line profile for representing high-resolution spectroscopic transitions (IUPAC Technical Report). Pure Appl Chem 2014;86:1931-43. doi:10.1515/pac-2014-0208.

[14] Ngo NH, Lisak D, Tran H, Hartmann J-M. An isolated line-shape model to go beyond the Voigt profile in spectroscopic databases and radiative transfer codes. J Quant Spectrosc Radiat Transf 2013;129:89-100. doi:10.1016/j.jqsrt. 2013.05.034. 
[15] Barber RJ, Tennyson J, Harris GJ, Tolchenov RN. A high-accuracy computed water line list. Mon Not R Astron Soc 2006;368:1087-94. doi:10.1111/j. 1365-2966.2006.10184.x.

[16] Lodi L, Tennyson J, Polyansky OL. A global, high accuracy ab initio dipole moment surface for the electronic ground state of the water molecule. J Chem Phys 2011;135:34113. doi:10.1063/1.3604934.

[17] Lodi L, Tennyson J. Line lists for $\mathrm{H}_{2}{ }^{18} \mathrm{O}$ and $\mathrm{H}_{2}{ }^{17} \mathrm{O}$ based on empirical line positions and $\mathrm{ab}$ initio intensities. J Quant Spectrosc Radiat Transf 2012;113:850-8. doi:10.1016/j.jqsrt.2012.02.023.

[18] Krasnopolsky VA, Belyaev DA, Gordon IE, Li G, Rothman LS. Observations of $\mathrm{D} / \mathrm{H}$ ratios in $\mathrm{H}_{2} \mathrm{O}, \mathrm{HCl}$, and $\mathrm{HF}$ on Venus and new $\mathrm{DCl}$ and $\mathrm{DF}$ line strengths. Icarus 2013;224:57-65. doi:10.1016/j.icarus.2013.02.010.

[19] Tennyson J, Bernath PF, Brown LR, Campargue A, Carleer MR, Császár AG, et al. IUPAC critical evaluation of the rotational-vibrational spectra of water vapor. Part I-Energy levels and transition wavenumbers for $\mathrm{H}_{2}{ }^{17} \mathrm{O}$ and $\mathrm{H}_{2}{ }^{18} \mathrm{O}$. J Quant Spectrosc Radiat Transf 2009;110:573-96. doi:10.1016/j.jqsrt.2009.02. 014

[20] Tennyson J, Bernath PF, Brown LR, Campargue A, Császár AG, Daumont L, et al. IUPAC critical evaluation of the rotational-vibrational spectra of water vapor. Part II. J Quant Spectrosc Radiat Transf 2010;111:2160-84. doi:10.1016 j.jqsrt.2010.06.012.

[21] Tennyson J, Bernath PF, Brown LR, Campargue A, Császár AG, Daumont L, et al. IUPAC critical evaluation of the rotational-vibrational spectra of water vapor, Part III: energy levels and transition wavenumbers for $\mathrm{H}_{2}{ }^{16} \mathrm{O}$. J Quant Spectrosc Radiat Transf 2013;117:29-58. doi:10.1016/j.jqsrt.2012.10.002.

[22] Tennyson J, Bernath PF, Brown LR, Campargue A, Császár AG, Daumont L, et al. IUPAC critical evaluation of the rotational-vibrational spectra of water vapor. Part IV. Energy levels and transition wavenumbers for $\mathrm{D}_{2}{ }^{16} \mathrm{O}, \mathrm{D}_{2}{ }^{17} \mathrm{O}$ and $\mathrm{D}_{2}{ }^{18} \mathrm{O}$. J Quant Spectrosc Radiat Transf 2014;142:93-108. doi:10.1016/j. jqsrt.2014.03.019,

[23] Mikhailenko SN, Kassi S, Mondelain D, Gamache RR, Campargue A. A spectroscopic database for water vapor between 5850 and $8340 \mathrm{~cm}^{-1}$. J Quant Spectrosc Radiat Transf 2016;179:198-216. doi:10.1016/j.jqsrt.2016.03.035.

[24] Liu A-W, Naumenko OV, Kassi S, Campargue A. CW-Cavity ring down spectroscopy of deuterated water in the $1.58 \mu \mathrm{m}$ atmospheric transparency window. J Quant Spectrosc Radiat Transf 2014;138:97-106. doi:10.1016/j.jqsrt. 2014.02.002

[25] Kyuberis AA, Zobov NF, Naumenko OV, Voronin BA, Polyansky OL, Lodi L et al. Room temperature linelists for deuterated water. J Quant Spectrosc Radiat Transf 2017 this issue. http://doi.org/10.1016/j.jqsrt.2017.06.026.

[26] Mikhailenko SN, Serdyukov VI, Sinitsa LN. LED-based Fourier transform spectroscopy of $\mathrm{H}_{2}{ }^{18} \mathrm{O}$ in the $15,000-16,000 \mathrm{~cm}^{-1}$ range. J Quant Spectrosc Radiat Transf 2015;156:36-46. doi:10.1016/j.jqsrt.2015.02.001.

[27] Regalia L, Oudot C, Mikhailenko S, Wang L, Thomas X, Jenouvrier A, et al. Water vapor line parameters from 6450 to $9400 \mathrm{~cm}^{-1}$. J Quant Spectrosc Radiat Transf 2014;136:119-36. doi:10.1016/j.jqsrt.2013.11.019.

[28] Mikhailenko S, Régalia L. Inventory of data included in HITRAN2012 edition for water vapor between 6450 and $9400 \mathrm{~cm}^{-1}$. J Mol Spectrosc 2016;327:159-70. doi:10.1016/j.jms.2016.01.001.

[29] Campargue A, Mikhailenko SN, Vasilchenko S, Reynaud C, Béguier S, Čermák P, et al. The absorption spectrum of water vapor in the $2.2 \mu \mathrm{m}$ transparency window: High sensitivity measurements and spectroscopic database. J Quant Spectrosc Radiat Transf 2017;189:407-16. doi:10.1016/j.jqsrt.2016.12. 016.

[30] Ponomarev YN, Solodov AA, Solodov AM, Petrova TM, Naumenko OV. FTIR spectrometer with $30 \mathrm{~m}$ optical cell and its applications to the sensitive measurements of selective and nonselective absorption spectra. J Quant Spectrosc Radiat Transf 2016;177:253-60. doi:10.1016/j.jqsrt.2016.02.026.

[31] Birk M, Wagner G, Loos J, Lodi L, Polyansky OL, Kyuberis AA, et al. Accurate line intensities for water transitions in the infrared: Comparison of theory and experiment. J Quant Spectrosc Radiat Transf 2017 This issue:HITRAN Special issue. doi:10.1016/j.jqsrt.2017.03.040.

[32] Wunch D, Toon GC, Blavier J-FL, Washenfelder RA, Notholt J, Connor BJ, et al The total carbon column observing network. Philos Trans R Soc A Math Phys Eng Sci 2011;369:2087-112. doi:10.1098/rsta.2010.0240.

[33] Loos J, Birk M, Wagner G. Measurement of air-broadening line shape parameters and temperature dependence parameters of $\mathrm{H}_{2} \mathrm{O}$ lines in the spectral ranges $1850-2280 \mathrm{~cm}^{-1}$ and $2390-4000 \mathrm{~cm}^{-1}$. J Quant Spectrosc Radiat Transf 2017 HITRAN Spe. doi:10.1016/j.jqsrt.2017.03.033.

[34] Loos J, Birk M, Wagner G. Measurement of position, intensities and selfbroadened line shape parameters of $\mathrm{H} 2 \mathrm{O}$ lines in the spectral ranges 1850 $2280 \mathrm{~cm}^{-1}$ and $2390-4000 \mathrm{~cm}^{-1}$. J Quant Spectrosc Radiat Transf 2017 HITRAN Spe. 10.1016/j.jqsrt.2017.02.013.

[35] Rosenkranz P. Shape of the $5 \mathrm{~mm}$ oxygen band in the atmosphere. IEEE Trans Antennas Propag 1975;23:498-506. doi:10.1109/TAP.1975.1141119.

[36] Veefkind JP, Aben I, McMullan K, Förster H, de Vries J, Otter G, et al. TROPOMI on the ESA Sentinel-5 Precursor: A GMES mission for global observations of the atmospheric composition for climate, air quality and ozone layer applications. Remote Sens Environ 2012;120:70-83. doi:10.1016/j.rse.2011.09.027.

[37] Leshchishina O, Mikhailenko S, Mondelain D, Kassi S, Campargue A. CRDS of water vapor at 0.1 Torr between 6886 and $7406 \mathrm{~cm}^{-1}$. J Quant Spectrosc Radiat Transf 2012;113:2155-66. doi:10.1016/j.jqsrt.2012.06.026.

[38] Campargue A, Mikhailenko SN, Lohan BG, Karlovets EV, Mondelain D, Kassi S The absorption spectrum of water vapor in the $1.25 \mu \mathrm{m}$ atmospheric window (7911-8337 $\mathrm{cm}^{-1}$ ). J Quant Spectrosc Radiat Transf 2015;157:135-52. doi:10. 1016/j.jqsrt.2015.02.011.
[39] Mikhailenko S, Kassi S, Wang L, Campargue A. The absorption spectrum of water in the $1.25 \mu \mathrm{m}$ transparency window $\left(7408-7920 \mathrm{~cm}^{-1}\right)$. J Mol Spectrosc 2011;269:92-103. doi:10.1016/j.jms.2011.05.005.

[40] Mikhailenko SN, Mondelain D, Kassi S, Campargue A. An accurate and complete empirical line list for water vapor between 5850 and $7920 \mathrm{~cm}^{-1}$ J Quant Spectrosc Radiat Transf 2014;140:48-57. doi:10.1016/j.jqsrt.2014.02. 006 .

[41] Leshchishina O, Mikhailenko SN, Mondelain D, Kassi S, Campargue A. An improved line list for water vapor in the $1.5 \mu \mathrm{m}$ transparency window by highly sensitive CRDS between 5852 and $6607 \mathrm{~cm}^{-1}$. J Quant Spectrosc Radiat Transf 2013;130:69-80. doi:10.1016/j.jqsrt.2013.04.010.

[42] Sironneau VT, Hodges JT. Line shapes, positions and intensities of water transitions near $1.28 \mu \mathrm{m}$. J Quant Spectrosc Radiat Transf 2015;152:1-15. doi:10. 1016/j.jqsrt.2014.10.020

[43] Toth RA. Linelist of water vapor parameters from 500 to $8000 \mathrm{~cm}^{-1}$ http: //mark4sun.jpl.nasa.gov/h2o.html.

[44] Schwenke DW, Partridge $H$. Convergence testing of the analytic representation of an ab initio dipole moment function for water: improved fitting yields improved intensities. J Chem Phys 2000;113:6592-7. doi:10.1063/1. 1311392.

[45] Partridge H, Schwenke DW. The determination of an accurate isotope dependent potential energy surface for water from extensive ab initio calculations and experimental data. J Chem Phys 1997;106:4618-39. doi:10.1063/1.473987.

[46] Gordon IE, Rothman LS, Gamache RR, Jacquemart D, Boone C, Bernath PF, et al. Current updates of the water-vapor line list in HITRAN: a new "Diet" for air-broadened half-widths. J Quant Spectrosc Radiat Transf 2007; 108:389402. doi:10.1016/j.jqsrt.2007.06.009.

[47] Jenouvrier A, Daumont L, Régalia-Jarlot L, Tyuterev VG, Carleer M, Vandaele AC, et al. Fourier transform measurements of water vapor line parameters in the $4200-6600 \mathrm{~cm}^{-1}$ region. J Quant Spectrosc Radiat Transf 2007;105:326-55. doi:10.1016/j.jqsrt.2006.11.007.

[48] Armante R, Scott N, Crevoisier C, Capelle V, Crepeau L, Chédin A. Evaluation of spectroscopic databases through radiative transfer simulations compared to observations. Application to the validation of GEISA 2015 with IASI and TCCON. J Mol Spectrosc 2016;327:180-92. doi:10.1016/j.jms.2016.04.004.

[49] Jacquinet-Husson N, Armante R, Scott NA, Chédin A, Crépeau L, Boutammine $C$, et al. The 2015 edition of the GEISA spectroscopic database. J Mol Spectrosc 2016;327:31-72. doi:10.1016/j.jms.2016.06.007.

[50] Jacquemart D, Gamache R, Rothman LS. Semi-empirical calculation of airbroadened half-widths and air pressure-induced frequency shifts of watervapor absorption lines. J Quant Spectrosc Radiat Transf 2005;96:205-39. doi:10.1016/j.jqsrt.2004.11.018

[51] Birk M, Wagner G. Temperature-dependent air broadening of water in the $1250-1750 \mathrm{~cm}^{-1}$ range. J Quant Spectrosc Radiat Transf 2012;113:889-928. doi:10.1016/j.jqsrt.2011.12.013.

[52] Malathy Devi V, Rinsland CP, Benner DC, Smith MAH. Tunable diode laser measurements of air- and $\mathrm{N}_{2}$-broadened halfwidths in the $v_{2}$ band of $\mathrm{D}_{2} \mathrm{O}$. Appl Opt 1986;25:336. doi:10.1364/AO.25.000336.

[53] Rinsland CP, Smith MAH, Devi VM, Benner DC. Measurements of Lorentzbroadening coefficients and pressure-induced line-shift coefficients in the $v_{1}$ band of $\mathrm{HD}^{16} \mathrm{O}$ and the $\nu_{3}$ band of $\mathrm{D}_{2}{ }^{16} \mathrm{O}$. J Mol Spectrosc 1992;156:507-11. doi:10.1016/0022-2852(92)90251-I.

[54] Rinsland CP, Smith MAH, Malathy Devi V, Benner DC. Measurements of Lorentz-broadening coefficients and pressure-induced line shift coefficients in the $v_{2}$ band of $\mathrm{D}_{2}{ }^{16} \mathrm{O}$. J Mol Spectrosc 1991;150:173-83. doi:10.1016 0022-2852(91)90200-T.

[55] Toth RA. Air- and N2-Broadening Parameters of $\mathrm{HDO}$ and $\mathrm{D}_{2} \mathrm{O}, 709$ to $1936 \mathrm{~cm}^{-1}$. J Mol Spectrosc 1999;198:358-70. doi:10.1006/jmsp.1999.7966.

[56] Gamache RR, Fischer J. Half-widths of $\mathrm{H}_{2}{ }^{16} \mathrm{O}, \mathrm{H}_{2}{ }^{18} \mathrm{O}, \mathrm{H}_{2}{ }^{17} \mathrm{O}, \mathrm{HD}^{16} \mathrm{O}$, and $\mathrm{D}_{2}{ }^{16} \mathrm{O}$ : I. Comparison between isotopomers. J Quant Spectrosc Radiat Transf 2003;78:289-304. doi:10.1016/S0022-4073(02)00217-0.

[57] Gamache RR, Farese M, Renaud CL. A spectral line list for water isotopologues in the $1100-4100 \mathrm{~cm}^{-1}$ region for application to $\mathrm{CO}_{2}$-rich planetary atmospheres. J Mol Spectrosc 2016;326:144-50. doi:10.1016/j.jms.2015.09.001.

[58] Brown LR, Humphrey CM, Gamache RR. $\mathrm{CO}_{2}$-broadened water in the pure rotation and $v_{2}$ fundamental regions. J Mol Spectrosc 2007;246:1-21. doi:10. 1016/j.jms.2007.07.010.

[59] Lampel J, Pöhler D, Polyansky OL, Kyuberis AA, Zobov NF, Tennyson J, et al Detection of water vapour absorption around $363 \mathrm{~nm}$ in measured atmospheric absorption spectra and its effect on DOAS evaluations. Atmos Chem Phys 2017;17:1271-95. doi:10.5194/acp-17-1271-2017.

[60] Dupré P, Gherman T, Zobov NF, Tolchenov RN, Tennyson J. Continuous-wave cavity ringdown spectroscopy of the $8 v$ polyad of water in the 25195-25340 $\mathrm{cm}^{-1}$ range. J Chem Phys 2005;123:154307. doi:10.1063/1.2055247.

[61] Grechko M, Maksyutenko P, Zobov NF, Shirin SV, Polyansky OL, Rizzo TR, et al. Collisionally Assisted Spectroscopy of Water from 27000 to $34000 \mathrm{~cm}^{-1}$. Phys Chem A 2008;112:10539-45. doi:10.1021/jp805849q.

[62] Maksyutenko P, Muenter JS, Zobov NF, Shirin SV, Polyansky OL, Rizzo TR et al. Approaching the full set of energy levels of water. J Chem Phys 2007;126:241101. doi:10.1063/1.2748751.

[63] Zoogman P, Liu X, Suleiman RM, Pennington WF, Flittner DE, Al-Saadi JA, et al. Tropospheric emissions: Monitoring of pollution (TEMPO). J Quant Spectrosc Radiat Transf 2017;186:17-39. doi:10.1016/j.jqsrt.2016.05.008.

[64] Crisp D, Atlas R, Breon F-M, Brown L, Burrows J, Ciais P, et al. The Orbiting Carbon Observatory (OCO) mission. Adv Sp Res 2004;34:700-9. doi:10.1016/ j.asr.2003.08.062 
[65] Connor B, Bösch H, McDuffie J, Taylor T, Fu D, Frankenberg C, et al. Quantification of uncertainties in OCO-2 measurements of $\mathrm{XCO}_{2}$ : simulations and linear error analysis. Atmos Meas Tech 2016;9:5227-38. doi:10.5194/ amt-9-5227-2016.

[66] Wunch D, Wennberg PO, Osterman G, Fisher B, Naylor B, Roehl CM, et al. Comparisons of the Orbiting Carbon Observatory-2 (OCO-2) $\mathrm{XCO}_{2}$ measurements with TCCON. Atmos Meas Tech Discuss 2016:1-45. doi:10.5194/ amt-2016-227.

[67] Butz A, Guerlet S, Hasekamp O, Schepers D, Galli A, Aben I, et al. Toward accurate $\mathrm{CO}_{2}$ and $\mathrm{CH}_{4}$ observations from GOSAT. Geophys Res Lett 2011;38:L14812. doi:10.1029/2011GL047888

[68] Ravi Kumar K, Valsala V, Tiwari YK, Revadekar JV, Pillai P, Chakraborty S, et al. Intra-seasonal variability of atmospheric $\mathrm{CO}_{2}$ concentrations over India during summer monsoons. Atmos Environ 2016;142:229-37. doi:10.1016 j.atmosenv.2016.07.023.

[69] Abshire JB, Riris H, Allan GR, Weaver CJ, Mao J, Sun X, et al. In: Singh UN, Pappalardo $\mathrm{G}$, editors. A lidar approach to measure $\mathrm{CO}_{2}$ concentrations from space for the ASCENDS mission; 2010. 78320D. doi: 10.1117/12.868567.

[70] Hase F. Improved instrumental line shape monitoring for the ground-based, high-resolution FTIR spectrometers of the network for the detection of atmospheric composition change. Atmos Meas Tech 2012;5:603-10. doi:10.5194/ amt-5-603-2012.

[71] Emmert JT, Stevens MH, Bernath PF, Drob DP, Boone CD. Observations of increasing carbon dioxide concentration in Earth's thermosphere. Nat Geosci 2012;5:868-71. doi:10.1038/ngeo1626.

[72] Wunch D, Wennberg PO, Toon GC, Connor BJ, Fisher B, Osterman GB, et al. A method for evaluating bias in global measurements of $\mathrm{CO}_{2}$ total columns from space. Atmos Chem Phys 2011;11:12317-37. doi:10.5194/ acp-11-12317-2011.

[73] Miller CE, Crisp D, DeCola PL, Olsen SC, Randerson JT, Michalak AM, et al. Precision requirements for space-based $\mathrm{X}-\mathrm{CO}_{2}$ data. J Geophys Res 2007;112:D10314. doi:10.1029/2006JD007659.

[74] Giusfredi G, Bartalini S, Borri S, Cancio P, Galli I, Mazzotti D, et al. Saturatedabsorption cavity ring-down spectroscopy. Phys Rev Lett 2010;104:110801. doi:10.1103/PhysRevLett.104.110801.

[75] Galli I, Bartalini S, Borri S, Cancio P, Mazzotti D, De Natale P, et al. Molecular Gas Sensing Below Parts Per Trillion: Radiocarbon-Dioxide Optical Detection. Phys Rev Lett 2011;107:270802. doi:10.1103/PhysRevLett.107. 270802.

[76] McCartt AD, Ognibene T, Bench G, Turteltaub K. Measurements of carbon-14 with cavity ring-down spectroscopy. Nucl Instrum Methods Phys Res Sect B Beam Interact with Mater Atoms 2015;361:277-80. doi:10.1016/j.nimb.2015. 05.036.

[77] Shved GM. On the abundances of carbon dioxide isotopologues in the atmospheres of mars and earth. Sol Syst Res 2016;50:161-4. doi:10.1134/ S0038094616020064.

[78] Perevalov VI, Tashkun SA. CDSD-296 (Carbon Dioxide Spectroscopic Databank): Updated and Enlarged Version for Atmospheric Applications. 2008. doi:10.5281/zenodo.17520.

[79] Huang X, Gamache RR, Freedman RS, Schwenke DW, Lee TJ. Reliable infrared line lists for $13 \mathrm{CO}_{2}$ isotopologues up to $\mathrm{E}^{\prime}=18,000 \mathrm{~cm}-1$ and $1500 \mathrm{~K}$, with line shape parameters. J Quant Spectrosc Radiat Transf 2014;147:134-44. doi:10.1016/j.jqsrt.2014.05.015.

[80] Vasilchenko S, Konefal M, Mondelain D, Kassi S, Čermák P, Tashkun SA, et al. The $\mathrm{CO}_{2}$ absorption spectrum in the $2.3 \mu \mathrm{m}$ transparency window by high sensitivity CRDS: (I) Rovibrational lines. J Quant Spectrosc Radiat Transf 2016;184:233-40. doi:10.1016/j.jqsrt.2016.07.002.

[81] Karlovets EV, Perevalov VI. The influence of isotopic substitution on the effective dipole moment parameters of $\mathrm{CO}_{2}$ molecule. Opt Spectrosc 2015;119:1621. doi:10.1134/S0030400X15070139.

[82] Zak E, Tennyson J, Polyansky OL, Lodi L, Zobov NF, Tashkun SA, et al. A room temperature $\mathrm{CO}_{2}$ line list with ab initio computed intensities. J Quant Spectrosc Radiat Transf 2016;177:31-42. doi:10.1016/j.jqsrt.2015.12.022.

[83] Zak EJ, Tennyson J, Polyansky OL, Lodi L, Zobov NF, Tashkun SA, et al. Room temperature line lists for $\mathrm{CO}_{2}$ symmetric isotopologues with ab initio computed intensities. J Quant Spectrosc Radiat Transf 2017;189:267-80. doi:10.1016/j.jqsrt.2016.11.022.

[84] Devi VM, Benner DC, Sung K, Brown LR, Crawford TJ, Miller CE, et al. Line parameters including temperature dependences of self- and air-broadened line shapes of ${ }^{12} \mathrm{C}^{16} \mathrm{O}_{2}$ : $1.6-\mu \mathrm{m}$ region. J Quant Spectrosc Radiat Transf 2016;177:117-44. doi:10.1016/j.jqsrt.2015.12.020

[85] Polyansky OL, Bielska K, Ghysels M, Lodi L, Zobov NF, Hodges JT, et al. HighAccuracy $\mathrm{CO}_{2}$ Line Intensities Determined from Theory and Experiment. Phys Rev Lett 2015;114:243001. doi:10.1103/PhysRevLett.114.243001.

[86] Benner DC, Devi VM, Sung K, Brown LR, Miller CE, Payne VH, et al. Line parameters including temperature dependences of air- and self-broadened line shapes of ${ }^{12} \mathrm{C}^{16} \mathrm{O}_{2}$ : 2.06- $\mu \mathrm{m}$ region. J Mol Spectrosc 2016;326:21-47. doi:10.1016/j.jms.2016.02.012

[87] Jacquemart D, Gueye F, Lyulin OM, Karlovets EV, Baron D, Perevalov VI. Infrared spectroscopy of $\mathrm{CO}_{2}$ isotopologues from 2200 to $7000 \mathrm{~cm}^{-1}$ : ICharacterizing experimental uncertainties of positions and intensities. J Quant Spectrosc Radiat Transf 2012;113:961-75. doi:10.1016/j.jqsrt.2012.02. 020.

[88] Toth RA, Miller CE, Brown LR, Devi VM, Benner DC. Line strengths of ${ }^{16} \mathrm{O}^{13} \mathrm{C}^{16} \mathrm{O},{ }^{16} \mathrm{O}^{13} \mathrm{C}^{18} \mathrm{O},{ }^{16} \mathrm{O}^{13} \mathrm{C}^{17} \mathrm{O}$ and ${ }^{18} \mathrm{O}^{13} \mathrm{C}^{18} \mathrm{O}$ between 2200 and $6800 \mathrm{~cm}^{-1}$. J Mol Spectrosc 2008;251:64-89. doi:10.1016/j.jms.2008.01.009.
[89] Toth RA, Miller CE, Brown LR, Devi VM, Benner DC. Line positions and strengths of ${ }^{16} \mathrm{O}^{12} \mathrm{C}^{18} \mathrm{O},{ }^{18} \mathrm{O}^{12} \mathrm{C}^{18} \mathrm{O}$ and ${ }^{17} \mathrm{O}^{12} \mathrm{C}^{18} \mathrm{O}$ between 2200 and $7000 \mathrm{~cm}^{-1}$. J Mol Spectrosc 2007;243:43-61. doi:10.1016/j.jms.2007.03. 005

[90] Toth RA, Brown LR, Miller CE, Malathy Devi V, Benner DC. Spectroscopic database of $\mathrm{CO}_{2}$ line parameters: $4300-7000 \mathrm{~cm}^{-1}$. J Quant Spectrosc Radiat Transf 2008;109:906-21. doi:10.1016/j.jqsrt.2007.12.004.

[91] Zak EJ, Tennyson J, Polyansky OL, Lodi L, Zobov NF, Tashkun SA, et al. Room temperature linelists for $\mathrm{CO}_{2}$ asymmetric isotopologues with ab initio computed intensities. J Quant Spectrosc Radiat Transf 2017. doi:10.1016/j.jqsrt. 2017.01.037.

[92] Tashkun SA, Perevalov VI, Gamache RR, Lamouroux J. CDSD-296, high resolution carbon dioxide spectroscopic databank: version for atmospheric applications. J Quant Spectrosc Radiat Transf 2015;152:45-73. doi:10.1016/j.jqsrt. 2014.10.017

[93] Schwenke DW, Tashkun SA, Lee TJ. An isotopic-independent highly accurate potential energy surface for $\mathrm{CO}_{2}$ isotopologues and an initial ${ }^{12} \mathrm{C}^{16} \mathrm{O}_{2}$ infrared line list. J Chem Phys 2012;136:124311. doi:10.1063/1.3697540.

[94] Huang X, Freedman RS, Tashkun SA, Schwenke DW, Lee TJ. Semi-empirical ${ }^{12} \mathrm{C}^{16} \mathrm{O}_{2}$ IR line lists for simulations up to $1500 \mathrm{~K}$ and $20,000 \mathrm{~cm}^{-1}$. J Quant Spectrosc Radiat Transf 2013;130:134-46. doi:10.1016/j.jqsrt.2013.05.018.

[95] Lyulin OM, Karlovets EV, Jacquemart D, Lu Y, Liu AW, Perevalov VI. Infrared spectroscopy of ${ }^{17} \mathrm{O}$ - and ${ }^{18} \mathrm{O}$-enriched carbon dioxide in the $1700-8300 \mathrm{~cm}^{-1}$ wavenumber region. J Quant Spectrosc Radiat Transf 2012;113:2167-81. doi:10.1016/j.jqsrt.2012.06.028.

[96] Karlovets EV, Campargue A, Mondelain D, Béguier S, Kassi S, Tashkun SA, et al. High sensitivity cavity ring down spectroscopy of ${ }^{18} \mathrm{O}$ enriched carbon dioxide between 5850 and $7000 \mathrm{~cm}^{-1}$ : I. Analysis and theoretical modeling of the ${ }^{16} \mathrm{O}^{12} \mathrm{C}^{18} \mathrm{O}$ spectrum. J Quant Spectrosc Radiat Transf 2013;130:116-33. doi:10.1016/j.jqsrt.2013.05.019.

[97] Karlovets EV, Campargue A, Mondelain D, Kassi S, Tashkun SA, Perevalov VI. High sensitivity cavity ring down spectroscopy of ${ }^{18} \mathrm{O}$ enriched carbon dioxide between 5850 and $7000 \mathrm{~cm}^{-1}$ : Part II-analysis and theoretical modeling of the ${ }^{12} \mathrm{C}^{18} \mathrm{O}_{2},{ }^{13} \mathrm{C}^{18} \mathrm{O}_{2}$. J Quant Spectrosc Radiat Transf 2014;136:71-88. doi:10.1016/j.jqsrt.2013.11.005.

[98] Campargue A, Song KF, Mouton N, Perevalov VI, Kassi S. High sensitivity CWcavity ring down spectroscopy of five ${ }^{13} \mathrm{CO}_{2}$ isotopologues of carbon dioxide in the 1.26-1.44 $\mu \mathrm{m}$ region (I): line positions. J Quant Spectrosc Radiat Transf 2010;111:659-74. doi:10.1016/j.jqsrt.2009.11.013.

[99] Durry G, Li JS, Vinogradov I, Titov A, Joly L, Cousin J, et al. Near infrared diode laser spectroscopy of $\mathrm{C}_{2} \mathrm{H}_{2}, \mathrm{H}_{2} \mathrm{O}, \mathrm{CO}_{2}$ and their isotopologues and the application to TDLAS, a tunable diode laser spectrometer for the martian PHOBOS-GRUNT space mission. Appl Phys B 2010;99:339-51. doi:10.1007/ s00340-010-3924-y

[100] Toth RA, Brown LR, Miller CE, Devi VM, Benner DC. Line strengths of ${ }^{12} \mathrm{C}^{16} \mathrm{O}_{2}$ : 4550-7000 $\mathrm{cm}^{-1}$. J Mol Spectrosc 2006;239:221-42. doi:10.1016/j.jms.2006. 08.001.

[101] Miller CE, Brown LR, Toth RA, Benner DC, Malathy Devi V. Near-Infrared Carbon Dioxide Spectral Database, The 10th HITRAN Database Conference Cambrifge, MA, June 2008. http://dx.doi.org/10.5281/zenodo.16555.

[102] Wübbeler G, Víquez GJP, Jousten K, Werhahn O, Elster C. Comparison and assessment of procedures for calculating the $\mathrm{R}(12)$ line strength of the $v_{1}+2 v_{2}+v_{3}$ band of $\mathrm{CO}_{2}$. J Chem Phys 2011;135:204304. doi:10.1063/ 1.3662134 .

[103] Odintsova TA, Fasci E, Moretti L, Zak EJ, Polyansky OL, Tennyson J, et al. Highly accurate intensity factors of pure $\mathrm{CO}_{2}$ lines near $2 \mu \mathrm{m}$. J Chem Phys 2017;146:244309. doi:10.1063/1.4989925.

[104] Hodges J.T. in preparation. 2017.

[105] Petrova TM, Solodov AM, Solodov AA, Lyulin OM, Tashkun SA, Perevalov VI. Measurements of ${ }^{12} \mathrm{CO}_{2}$ line parameters in the 8790-8860, 9340-9650 and $11,430-11,505 \mathrm{~cm}^{-1}$ wavenumber regions by means of Fourier transform spectroscopy. J Quant Spectrosc Radiat Transf 2013;124:21-7. doi:10.1016/j. jqsit.2013.03.017.

[106] Petrova TM, Solodov AM, Solodov AA, Lyulin OM, Borkov YG, Tashkun SA, et al. Measurements of $\mathrm{CO}_{2}$ line parameters in the $9250-9500 \mathrm{~cm}^{-1}$ and 10,700-10,860 $\mathrm{cm}^{-1}$ regions. J Quant Spectrosc Radiat Transf 2015;164:10916. doi:10.1016/j.jqsrt.2015.06.001

[107] Tan Y, Zhao X-Q Liu A-W, Hu S-M, Lyulin OM, Tashkun SA, et al. Cavity ringdown spectroscopy of $\mathrm{CO}_{2}$ overtone bands near $830 \mathrm{~nm}$. J Quant Spectrosc Radiat Transf 2015;165:22-7. doi:10.1016/j.jqsrt.2015.06.010.

[108] Solodov AM, Petrova TM, Solodov AA, Borkov YG, Tashkun SA, Perevalov VI. Measurements of the $\mathrm{CO}_{2}$ line parameters in the $10000-10300 \mathrm{~cm}^{-1}$ region. J Quant Spectrosc Radiat Transf 2017 this issue. http://doi.org/10.1016/j.jqsrt. 2017.04.012

[109] Majcherova Z, Macko P, Romanini D, Perevalov VI, Tashkun SA, Teffo J-L, et al. High-sensitivity CW-cavity ringdown spectroscopy of ${ }^{12} \mathrm{CO}_{2}$ near $1.5 \mu \mathrm{m}$. J Mol Spectrosc 2005:230:1-21. doi:10.1016/j.jms.2004.09.011.

[110] Karlovets EV, Campargue A, Mondelain D, Kassi S, Tashkun SA, Perevalov VI. High sensitivity cavity ring down spectroscopy of ${ }^{18} \mathrm{O}$ enriched carbon dioxide between 5850 and $7000 \mathrm{~cm}^{-1}$ : Part III-Analysis and theoretical modeling of the ${ }^{12} \mathrm{C}^{17} \mathrm{O}_{2},{ }^{16} \mathrm{O}^{12} \mathrm{C}^{17} \mathrm{O}$. J Quant Spectrosc Radiat Transf 2014;136:89-107. doi:10.1016/j.jqsrt.2013.11.006

[111] Kassi S, Karlovets EV, Tashkun SA, Perevalov VI, Campargue A. Analysis and theoretical modeling of the ${ }^{18} \mathrm{O}$ enriched carbon dioxide spectrum by CRDS

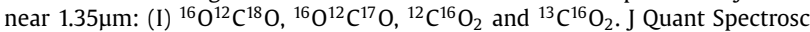
Radiat Transf 2017;187:414-25. doi:10.1016/j.jqsrt.2016.09.002. 
[112] Laraia AL, Gamache RR, Lamouroux J, Gordon IE, Rothman LS. Total internal partition sums to support planetary remote sensing. Icarus 2011;215:391400. doi:10.1016/j.icarus.2011.06.004.

[113] Oyafuso F, Payne VH, Drouin BJ, Devi VM, Benner DC, Sung K, et al. High accuracy absorption coefficients for the Orbiting Carbon Observatory-2 (OCO2) mission: Validation of updated carbon dioxide cross-sections using atmospheric spectra. J Quant Spectrosc Radiat Transf 2017 submitted:this issue. doi:10.1016/j.jqsrt.2017.06.012.

[114] Gamache RR, Lamouroux J. Predicting accurate line shape parameters for $\mathrm{CO}_{2}$ transitions. J Quant Spectrosc Radiat Transf 2013;130:158-71. doi:10.1016/j. jqsit.2013.05.021.

[115] Lamouroux J, Gamache RR, Laraia AL, Hartmann J-M, Boulet C. Semiclassical calculations of half-widths and line shifts for transitions in the $30012 \leftarrow 00001$ and $30013 \leftarrow 00001$ bands of $\mathrm{CO}_{2}$. III: Self collisions. J Quant Spectrosc Radiat Transf 2012;113:1536-46. doi:10.1016/j.jqsrt.2012.03.035.

[116] Lamouroux J, Gamache RR, Laraia AL, Hartmann J-M, Boulet C. Semiclassical calculations of half-widths and line shifts for transitions in the $30012 \leftarrow 00001$ and $30013 \leftarrow 00001$ bands of $\mathrm{CO}_{2}$ II: Collisions with $\mathrm{O}_{2}$ and air. J Quant Spectrosc Radiat Transf 2012;113:991-1003. doi:10.1016/j.jqsit.2012. 02.015 .

[117] Lamouroux J, Régalia L, Thomas X, Vander Auwera J, Gamache RR, Hartmann JM. $\mathrm{CO}_{2}$ line-mixing database and software update and its tests in the $2.1 \mu \mathrm{m}$ and $4.3 \mu \mathrm{m}$ regions. J Quant Spectrosc Radiat Transf 2015;151:88-96. doi:10.1016/j.jqsit.2014.09.017.

[118] Hedelt P, von Paris P, Godolt M, Gebauer S, Grenfell JL, Rauer H, et al Spectral features of earth-like planets and their detectability at different orbital distances around F, G, and K-type stars. Astron Astrophys 2013;9:15. doi:10.1051/0004-6361/201117723.

[119] Babikov YL, Mikhailenko SN, Barbe A, Tyuterev VG. S\&MPO-An information system for ozone spectroscopy on the WEB. J Quant Spectrosc Radiat Transf 2014;145:169-96. doi:10.1016/j.jqsrt.2014.04.024.

[120] Barbe A, Mikhailenko S, Starikova E, De Backer M-R, Tyuterev VG, Mondelain D, et al. Ozone spectroscopy in the electronic ground state: Highresolution spectra analyses and update of line parameters since 2003. J Quant Spectrosc Radiat Transf 2013;130:172-90. doi:10.1016/j.jqsrt.2013.06.007.

[121] Mikhailenko SN. Studies of ozone absorption spectra between 2000 and 2015. Atmos Ocean Opt 2015;28:587-606.

[122] Rixon G., Dubernet M.L., Piskunov N., Walton N., Mason N., Le Sidaner P., et al. VAMDC-The Virtual Atomic and Molecular Data Centre-A New Way to Disseminate Atomic and Molecular Data-VAMDC Level 1 Release, 2011, p. 107-15. doi:10.1063/1.3585810.

[123] Dubernet ML, Antony BK, Ba YA, Babikov YL, Bartschat K, Boudon V, et al. The virtual atomic and molecular data centre (VAMDC) consortium. J Phys B At Mol Opt Phys 2016;49:74003. doi:10.1088/0953-4075/49/7/074003

[124] Picquet-Varrault B, Orphal J, Doussin J-F, Carlier P, Flaud J-M. Laboratory intercomparison of the ozone absorption coefficients in the mid-infrared $(10 \mu \mathrm{m})$ and ultraviolet $(300-350 \mathrm{~nm})$ spectral regions. J Phys Chem A 2005;109:1008-14. doi:10.1021/jp0405411.

[125] Janssen C, Boursier C, Jeseck P, Té Y. Line parameter study of ozone at 5 and $10 \mu \mathrm{m}$ using atmospheric FTIR spectra from the ground: A spectroscopic database and wavelength region comparison. J Mol Spectrosc 2016;326:4859. doi:10.1016/j.jms.2016.04.003.

[126] Flaud J-M, Camy-Peyret C, Rinsland CP, Smith MAH, Malathy Devi V. Atlas of ozone line parameters from microwave to medium infrared. New York: Academic Press; 1990

[127] Barbe A, Plateaux JJ, Bouazza S, Sulakshina O, Mikhailenko S, Tyuterev V, et al. Experimental and theoretical study of absolute intensities of ozone spectral lines in the range $1850-2300 \mathrm{~cm}^{-1}$. J Quant Spectrosc Radiat Transf 1994;52:341-55. doi:10.1016/0022-4073(94)90164-3.

[128] Tyuterev VG, Barbe A, Mikhailenko SN, Tashkun SA. Ozone $5 \mu \mathrm{m}$ range revisited: accurate laboratory measurements and ab initio calculations. 13th HITRAN Database Conf.; 2014

[129] Goldman A, Barbe A, Tyuterev VG, De Backer-Barilly M-R, Hannigan JW, Coffey MT, et al. Identification of enhanced absorption by ${ }^{16} \mathrm{O}_{3}$ lines around $5 \mu \mathrm{m}$ in high-resolution FTIR solar spectra. J Quant Spectrosc Radiat Transf 2005:96:241-50. doi:10.1016/j.jqsrt.2004.10.009.

[130] Wagner G, Birk M, Schreier F, Flaud JM. Spectroscopic database for ozone in the fundamental spectral regions. J Geophys Res Atmos 2002;107:1-18. doi:10.1029/2001JD000818.

[131] Flaud J-M, Piccolo C, Carli B, Perrin A, Coudert LH, Teffo JL, et al. Molecular line parameters for the MIPAS (Michelson Interferometer for Passive Atmospheric Sounding) experiment. Atmos Ocean Opt 2003;16:172-82.

[132] Barbe A, Sulakshina O, Plateaux JJ. Hamdouni A, Bouazza S. High-resolution infrared spectra of ozone in the $2300-2600 \mathrm{~cm}^{-1}$ Region. J Mol Spectrosc 1995;170:244-50. doi:10.1006/jmsp.1995.1068.

[133] Tyuterev VG, Kochanov R V, Tashkun SA. Accurate $a b$ initio dipole moment surfaces of ozone: first principle intensity predictions for rotationally resolved spectra in a large range of overtone and combination bands. J Chem Phys 2017;146:64304. doi:10.1063/1.4973977.

[134] Tyuterev VG, Kochanov R V, Tashkun SA, Holka F, Szalay PG. New analytical model for the ozone electronic ground state potential surface and accurate $a b$ initio vibrational predictions at high energy range. J Chem Phys 2013;139:134307. doi:10.1063/1.4821638.

[135] Bouazza S, Mikhailenko S, Barbe A, Regalia L, Tyuterev VG, Plateaux JJ. The $v_{1}+v_{2}+2 v_{3}$ and $v_{2}+3 v_{3}$ Bands of ${ }^{16} \mathrm{O}_{3}$. J Mol Spectrosc 1995;174:510-19. doi:10.1006/jmsp.1995.0019.
[136] Barbe A, Sulakshina O, Plateaux JJ, Tyuterev VG, Bouazza S. Line Positions and Intensities of the $3 v_{1}+v_{3}$ Band of Ozone. J Mol Spectrosc 1996;175:296-302. doi:10.1006/jmsp.1996.0035.

[137] Barbe A, Mikhailenko S, Tyuterev V, Hamdouni A, Plateaux JJ. Analysis of the $2 v_{1}+2 v_{2}+v_{3}$ Band of Ozone. J Mol Spectrosc 1995;171:583-8. doi:10.1006/ jmsp.1995.1147.

[138] Barbe A., Mikhailenko S.N. Unpublished results. 2017.

[139] Flaud J-M, Barbe A, Camy-Peyret C, Plateaux JJ. High resolution analysis of the $5 v_{3}, 3 v_{1}+v_{2}+v_{3}$, and $v_{1}+4 v_{3}$ bands of ${ }^{16} \mathrm{O}_{3}$ : line positions and intensities. Mol Spectrosc 1996;177:34-9. doi:10.1006/jmsp.1996.0114.

[140] Birk M, Wagner G, Flaud JM, Hausamann D. Line strengths in the $v_{3}-v_{2}$ hot band of ozone. J Mol Spectrosc 1994;163:262-75. doi:10.1006/jmsp.1994. 1022.

[141] Flaud JM, Wagner G, Birk M, Camy-Peyret C, Claveau C, De Backer-Barilly MR, et al. Ozone absorption around 10 $\mu \mathrm{m}$. J Geophys Res Atmos 2003;108:4269. doi:10.1029/2002JD002755.

142] Mikhailenko S, Barbe A, Tyuterev V. Extended analysis of line positions and intensities of ozone bands in the $2900-3400 \mathrm{~cm}^{-1}$ region. J Mol Spectrosc 2002;215:29-41. doi:10.1006/jmsp.2002.8597.

[143] Barbe A, De Backer M-R, Starikova E, Tashkun SA, Thomas X, Tyuterev VG. FTS high resolution spectra of ${ }^{16} \mathrm{O}_{3}$ in 3500 and $5500 \mathrm{~cm}^{-1}$ regions. First example of new theoretical modelling for a polyad of strongly coupled states. J Quant Spectrosc Radiat Transf 2012;113:829-39. doi:10.1016/j.jqsrt.2012.02.034.

[144] Mikhailenko S, Barbe A, Tyuterev VG, Regalia L, Plateaux JJ. Line positions and intensities of the $v_{1}+v_{2}+3 v_{3}, v_{2}+4 v_{3}$, and $3 v_{1}+2 v_{2}$ bands of ozone. J Mol Spectrosc 1996;180:227-35. doi:10.1006/jmsp.1996.0246.

[145] Barbe A, Plateaux JJ, Tyuterev VG, Mikhailenko S. Analysis of high resolution measurements of the $2 v_{1}+3 v_{3}$ band of ozone: coriolis interaction with the $v_{1}+3 v_{2}+2 v_{3}$ band. J Quant Spectrosc Radiat Transf 1998;59:185-94. doi:10. 1016/S0022-4073(97)00117-9.

[146] Toon G.C. Evaluation of HITRAN $2016 \mathrm{O}_{3}$ linelist 2017. http://mark4sun.jpl. nasa.gov/report/o3_spectroscopy_evaluation_20170117.pdf.

[147] Toon GC, Blavier J-F, Sung K, Rothman LS, Gordon IE. HITRAN spectroscopy evaluation using solar occultation FTIR spectra. J Quant Spectrosc Radiat Transf 2016;182:324-36. doi:10.1016/j.jqsrt.2016.05.021.

[148] Perrin A, Vasserot A-M, Flaud J-M, Camy-Peyret C, Malathy Devi V, Smith MAH, et al. The $2.5-\mu \mathrm{m}$ bands of ozone: line positions and intensities. J Mol Spectrosc 1991;149:519-29. doi:10.1016/0022-2852(91)90307-V.

[149] Campargue A, Kassi S, Romanini D, Barbe A, De Backer-Barilly M-R, Tyuterev VG. CW-cavity ring down spectroscopy of the ozone molecule in the $6625-6830 \mathrm{~cm}^{-1}$ region. J Mol Spectrosc 2006;240:1-13. doi:10.1016/j. jms.2006.07.010.

[150] Barbe A, De Backer-Barilly M-R, Tyuterev VG, Kassi S, Campargue A. Detection and analysis of new bands of ${ }^{16} \mathrm{O}_{3}$ by CRDS between 6500 and $7300 \mathrm{~cm}^{-1}$. J Mol Spectrosc 2011;269:175-86. doi:10.1016/j.jms.2011.06.005.

[151] Tyuterev VG, Kochanov R, Campargue A, Kassi S, Mondelain D, Barbe A, et al. Does the "Reef Structure" at the ozone transition state towards the dissociation exist? New insight from calculations and ultrasensitive spectroscopy experiments. Phys Rev Lett 2014;113:143002. doi:10.1103/PhysRevLett.113. 143002.

[152] Marcus RA. Theory of mass-independent fractionation of isotopes, phase space accessibility, and a role of isotopic symmetry. Proc Natl Acad Sci 2013;110:17703-7. doi:10.1073/pnas.1213080110.

[153] Tashkun SA, Perevalov VI, Karlovets EV, Kassi S, Campargue A. High sensitivity cavity ring down spectroscopy of $\mathrm{N}_{2} \mathrm{O}$ near $1.22 \mu \mathrm{m}$ : (II) ${ }^{14} \mathrm{~N}_{2}{ }^{16} \mathrm{O}$ line intensity modeling and global fit of ${ }^{14} \mathrm{~N}_{2}{ }^{18} \mathrm{O}$ line positions. J Quant Spectrosc Radiat Transf 2016;176:62-9. doi:10.1016/j.jqsrt.2016.02.020.

[154] Tashkun SA, Perevalov VI, Liu A-W, Hu S-M. Global modeling of the ${ }^{15} \mathrm{~N}_{2}{ }^{16} \mathrm{O}$ line positions within the framework of the polyad model of effective Hamiltonian and a room temperature ${ }^{15} \mathrm{~N}_{2}{ }^{16} \mathrm{O}$ line list. J Quant Spectrosc Radiat Transf 2016;175:1-7. doi:10.1016/j.jqsrt.2016.01.038.

[155] Perevalov VI, Tashkun SA, Kochanov RV, Liu A-W, Campargue A. Global modeling of the ${ }^{14} \mathrm{~N}_{2}{ }^{16} \mathrm{O}$ line positions within the framework of the polyad model of effective Hamiltonian. J Quant Spectrosc Radiat Transf 2012;113:1004-12. doi:10.1016/j.jqsrt.2011.12.008

[156] Karlovets EV, Perevalov VI. Calculation of the carbon dioxide effective dipole moment parameters of the $\mathrm{qJ}$ and $\mathrm{q}^{2} \mathrm{~J}$ types for rare isotopologues. Opt Atmos I Okeana (in Russ) 2011;24:101-6

[157] Tashkun SA, Perevalov VI, Lavrentieva NN. NOSD-1000, the high-temperature nitrous oxide spectroscopic databank. J Quant Spectrosc Radiat Transf 2016;177:43-8. doi:10.1016/j.jqsrt.2015.11.014

[158] Ding Y, Perevalov VI, Tashkun SA, Teffo J-L, Hu S, Bertseva E, et al. Weak overtone transitions of $\mathrm{N}_{2} \mathrm{O}$ around $1.05 \mu \mathrm{m}$ by ICLAS-VECSEL. J Mol Spectrosc 2003:220:80-6. doi:10.1016/S0022-2852(03)00060-2.

[159] Liu AW, Kassi S, Malara P, Romanini D, Perevalov VI, Tashkun SA, et al. High sensitivity CW-cavity ring down spectroscopy of $\mathrm{N}_{2} \mathrm{O}$ near $1.5 \mu \mathrm{m}$ (I). J Mo Spectrosc 2007;244:33-47. doi:10.1016/j.jms.2007.01.007.

[160] Toth R.A. Linelist of $\mathrm{N}_{2} \mathrm{O}$ parameters from 500 to $7500 \mathrm{~cm}^{-1}$. n.d. http:/ mark4sun.jpl.nasa.gov/n2o.html.

[161] Loos J, Birk M, Wagner G. Pressure broadening, -shift, speed dependence and line mixing in the $v_{3}$ rovibrational band of $\mathrm{N}_{2} \mathrm{O}$. I Quant Spectrosc Radiat Transf 2015;151:300-9. doi:10.1016/j.jqsrt.2014.10.008.

[162] Li G, Gordon IE, Rothman LS, Tan Y, Hu S-M, Kassi S, et al. Rovibrational line lists for nine isotopologues of the CO molecule in the $\mathrm{X}^{1} \Sigma^{+}$ground electronic state. Astrophys J Suppl Ser 2015;216:15. doi:10.1088/0067-0049/ $216 / 1 / 15$ 
[163] Li G, Gordon IE, Bernath PF, Rothman LS. Direct fit of experimental rovibrational intensities to the dipole moment function: Application to $\mathrm{HCl}$. J Quant Spectrosc Radiat Transf 2011;112:1543-50. doi:10.1016/j.jqsrt.2011.03. 014

[164] Coxon JA, Hajigeorgiou PG. Direct potential fit analysis of the $\mathrm{X}^{1} \Sigma^{+}$ground state of CO. J Chem Phys 2004;121:2992-3008. doi:10.1063/1.1768167.

[165] Le Roy RJ.. LEVEL: A computer program for solving the radial Schrödinger equation for bound and quasibound levels. J Quant Spectrosc Radiat Transf 2017;186:167-78. doi:10.1016/j.jqsrt.2016.05.028.

[166] Malathy Devi V, Chris Benner D, Smith MAH, Mantz AW, Sung K, Brown LR, et al. Spectral line parameters including temperature dependences of selfand air-broadening in the $2 \leftarrow 0$ band of CO at $2.3 \mu \mathrm{m}$. J Quant Spectrosc Radiat Transf 2012;113:1013-33. doi:10.1016/j.jqsrt.2012.02.010.

[167] Hartmann J-M, Boulet C. Line shape parameters for HF in a bath of argon as a test of classical path models. J Chem Phys 2000;113:9000. doi:10.1063/1. 1319346.

[168] Pollock CR, Petersen FR, Jennings DA, Wells JS, Maki AG. Absolute frequency measurements of the 2-0 band of $\mathrm{CO}$ at $2.3 \mu \mathrm{m}$; Calibration standard frequencies from high resolution color center laser spectroscopy. J Mol Spectrosc 1983;99:357-68. doi:10.1016/0022-2852(83)90320-X

[169] Mondelain D, Sala T, Kassi S, Romanini D, Marangoni M, Campargue A. Broadband and highly sensitive comb-assisted cavity ring down spectroscopy of CO near $1.57 \mu \mathrm{m}$ with sub-MHz frequency accuracy. J Quant Spectrosc Radiat Transf 2015;154:35-43. doi:10.1016/j.jqsrt.2014.11.021.

[170] Cygan A, Wójtewicz S, Kowzan G, Zaborowski M, Wcisło P, Nawrocki J, et al. Absolute molecular transition frequencies measured by three cavityenhanced spectroscopy techniques. J Chem Phys 2016;144:0-11. doi:10.1063/ 1.4952651.

[171] Tan Y, Wang J, Zhao X-Q, Liu A-W, Hu S-M. Cavity ring-down spectroscopy of the fifth overtone of CO. J Quant Spectrosc Radiat Transf 2017;187:274-9. doi:10.1016/j.jqsrt.2016.10.003.

[172] Malathy Devi V, Chris Benner D, Smith MAH, Mantz AW, Sung K, Brown LR. Spectral line parameters including temperature dependences of air-broadening for the $2 \leftarrow 0$ bands of ${ }^{13} \mathrm{C}^{16} \mathrm{O}$ and ${ }^{12} \mathrm{C}^{18} \mathrm{O}$ at $2.3 \mu \mathrm{m}$. J Mol Spectrosc 2012;276-277:33-48. doi:10.1016/j.jms.2012.05.005.

[173] Sinclair PM, Duggan P, Berman R, Drummond JR, May AD. Line broadening in the fundamental band of $\mathrm{CO}$ in $\mathrm{CO}-\mathrm{He}$ and $\mathrm{CO}-\mathrm{Ar}$ mixtures. J Mol Spectros 1998;191:258-64. doi:10.1006/jmsp.1998.7628.

[174] Picard-Bersellini A, Charneau R, Brechignac P. Pressure broadening of CO infrared lines perturbed by $\mathrm{H}_{2}$ and He. J Chem Phys 1983;78:5900. doi:10.1063/ 1.444594.

[175] Luo C, Wehr R, Drummond JR, May AD, Thibault F, Boissoles J, et al. Shifting and broadening in the fundamental band of $\mathrm{CO}$ highly diluted in $\mathrm{He}$ and $\mathrm{Ar}$ : a comparison with theory. J Chem Phys 2001;115:2198-206. doi:10.1063/1. 1383049

[176] Brown LR, Sung K, Benner DC, Devi VM, Boudon V, Gabard T, et al. Methane line parameters in the HITRAN2012 database. J Quant Spectrosc Radiat Transf 2013;130:201-19. doi:10.1016/j.jqsrt.2013.06.020.

[177] Kozlov DN, Sadovskii DA, Radi PP. Laser-induced grating spectroscopy of highly excited overtone and combination vibrational states of methane. J Mol Spectrosc 2013;291:23-32. doi:10.1016/j.jms.2013.04.005.

[178] Ulenikov ON, Bekhtereva ES, Albert S, Bauerecker S, Niederer HM, Quack M. Survey of the high resolution infrared spectrum of methane ${ }^{12} \mathrm{CH}_{4}$ and ${ }^{13} \mathrm{CH}_{4}$ ): Partial vibrational assignment extended towards $12000 \mathrm{~cm}^{-1}$. J Chem Phys 2014;141:234302. doi:10.1063/1.4899263.

[179] Devi VM, Benner DC, Sung K, Crawford TJ, Yu S, Brown LR, et al. Self- and airbroadened line shapes in the $2 \nu_{3} \mathrm{P}$ and $\mathrm{R}$ branches of ${ }^{12} \mathrm{CH}_{4}$. J Mol Spectrosc 2015;315:114-36. doi:10.1016/j.jms.2015.05.003.

[180] Rey M, Nikitin AV, Tyuterev VG. Convergence of normal mode variational calculations of methane spectra: Theoretical linelist in the icosad range computed from potential energy and dipole moment surfaces. J Quant Spectrosc Radiat Transf 2015;164:207-20. doi:10.1016/j.jqsrt.2015.06.009.

[181] Delahaye T, Maxwell SE, Reed ZD, Lin H, Hodges JT, Sung K, et al. Precise methane absorption measurements in the $1.64 \mu \mathrm{m}$ spectral region for the MERLIN mission. J Geophys Res Atmos 2016;121:7360-70. doi:10.1002/ 2016JD025024.

[182] Nikitin AV, Rey M, Tashkun SA, Kassi S, Mondelain D, Campargue A, et al. Analyses and modeling of the ${ }^{12} \mathrm{CH}_{4}$ spectrum at $80 \mathrm{~K}$ between 6539 and $6800 \mathrm{~cm}^{-1}$. J Quant Spectrosc Radiat Transf 2016;168:207-16. doi:10.1016/ j.jqsrt.2015.09.014

[183] Hashemi R, Predoi-Cross A, Nikitin AV, Tyuterev VG, Sung K, Smith MAH, et al. Spectroscopic line parameters of ${ }^{12} \mathrm{CH}_{4}$ for atmospheric composition retrievals in the $4300-4500 \mathrm{~cm}^{-1}$ region. J Quant Spectrosc Radiat Transf 2017;186:106-17. doi:10.1016/j.jqsrt.2016.03.024.

[184] Hargreaves RJ, Bernath PF, Bailey J, Dulick M. Empirical line lists and absorption cross sections for methane at high temperatures. Astrophys J 2015;813:12. doi:10.1088/0004-637X/813/1/12.

[185] Amyay B, Louviot M, Pirali O, Georges R, Vander Auwera J, Boudon V. Global analysis of the high temperature infrared emission spectrum of ${ }^{12} \mathrm{CH}_{4}$ in the dyad $\left(v_{2} / v_{4}\right)$ region. J Chem Phys 2016;144:24312. doi:10.1063/1.4939521.

[186] Ba YA, Wenger C, Surleau R, Boudon V, Rotger M, Daumont L, et al. MeCaSDa and ECaSDa: methane and ethene calculated spectroscopic databases for the virtual atomic and molecular data centre. J Quant Spectrosc Radiat Transf 2013;130:62-8. doi:10.1016/j.jqsrt.2013.05.001.

[187] Tyuterev V, Tashkun S, Rey M, Kochanov R, Nikitin A, Delahaye T. Accurate Spectroscopic Models for Methane Polyads Derived from a Potential
Energy Surface Using High-Order Contact Transformations. J Phys Chem A 2013;117:13779-805. doi:10.1021/jp408116j.

[188] Birk M, Wagner G, Loos J, Wilzewski J, Mondelain D, Campargue A et al. Methane and water spectroscopic database for TROPOMI/Sentinel-5 Precursor in the $2.3 \mu \mathrm{m}$ region EGU-2017, Vienna:; 2017.

[189] Devi VM, Benner DC, Smith MAH, Mantz AW, Sung K, Crawford TJ, et al. Selfand air-broadened line shape parameters in the $v_{2}+v_{3}$ band of ${ }^{12} \mathrm{CH}_{4}: 4500$ $4630 \mathrm{~cm}^{-1}$. J Quant Spectrosc Radiat Transf 2015;152:149-65. doi:10.1016/j. jqsit.2014.11.011.

[190] Nikitin AV, Thomas X, Régalia L, Daumont L, Rey M, Tashkun SA, et al. Measurements and modeling of long-path ${ }^{12} \mathrm{CH}_{4}$ spectra in the $4800-5300 \mathrm{~cm}^{-1}$ region. J Quant Spectrosc Radiat Transf 2014;138:116-23. doi:10.1016/j.jqsrt. 2014.02.005.

[191] Lyulin OM, Nikitin AV, Perevalov VI, Morino I, Yokota T, Kumazawa R, et al. Measurements of $\mathrm{N}_{2}$ - and $\mathrm{O}_{2}$-broadening and shifting parameters of methane spectral lines in the 5550-6236 $\mathrm{cm}^{-1}$ region. J Quant Spectrosc Radiat Transf 2009;110:654-68. doi:10.1016/j.jqsrt.2009.02.012.

[192] Nikitin A.V., Thomas X., Daumont L., Rey M., Sung K., Toon G.C., et al. Measurements and analysis of ${ }^{12} \mathrm{CH}_{4}$ spectra in the $5300-5550 \mathrm{~cm}^{-1}$ region 2017:in preparation.

[193] Nikitin AV, Lyulin OM, Mikhailenko SN, Perevalov VI, Filippov NN, Grigoriev IM, et al. GOSAT-2014 methane spectral line list. J Quant Spectrosc Radiat Transf 2015;154:63-71. doi:10.1016/j.jqsrt.2014.12.003.

[194] Nikitin AV, Chizhmakova IS, Rey M, Tashkun SA, Kassi S, Mondelain D, et al Analysis of the absorption spectrum of ${ }^{12} \mathrm{CH} 4$ in the region $5855-6250 \mathrm{~cm}^{-1}$ of the $2 v_{3}$ band. J Quant Spectrosc Radiat Transf 2017 this issue. doi:10.1016/ j.jqsrt.2017.05.014

[195] Starikova E, Nikitin AV, Rey M, Tashkun SA, Mondelain D, Kassi S, et al. Assignment and modeling of the absorption spectrum of ${ }^{13} \mathrm{CH}_{4}$ band (5853$6201 \mathrm{~cm}^{-1}$ ). J Quant Spectrosc Radiat Transf 2016;177:170-80. doi:10.1016/j. jqsrt.2015.12.023.

[196] Béguier S, Kassi S, Campargue A. An empirical line list for methane in the $1.25 \mu \mathrm{m}$ transparency window. J Mol Spectrosc 2015;308-309:1-5. doi:10. 1016/j.jms.2014.12.020.

[197] Béguier S, Liu AW, Campargue A. An empirical line list for methane near $1 \mu \mathrm{m}$ (9028-10,435 $\left.\mathrm{cm}^{-1}\right)$. J Quant Spectrosc Radiat Transf 2015;166:6-12. doi:10. 1016/j.jqsrt.2015.07.003.

[198] Rothman LS, Gordon IE, Barbe A, Benner DC, Bernath PF, Birk M, et al. The HITRAN 2008 molecular spectroscopic database. J Quant Spectrosc Radiat Transf 2009;110:533-72. doi:10.1016/j.jqsrt.2009.02.013.

[199] Tennyson J, Yurchenko SN, Al-Refaie AF, Barton EJ, Chubb KL, Coles PA, et al. The ExoMol database: Molecular line lists for exoplanet and other hot atmospheres. J Mol Spectrosc 2016;327:73-94. doi:10.1016/j.jms.2016.05. 002

[200] Rey M, Nikitin A V, Tyuterev VG. First principles intensity calculations of the methane rovibrational spectra in the infrared up to $9300 \mathrm{~cm}^{-1}$. Phys Chem Chem Phys 2013;15:10049. doi:10.1039/c3cp50275a.

[201] Rey M, Nikitin A V, Tyuterev VG. Theoretical hot methane line lists up to $\mathrm{T}=2000 \mathrm{~K}$ for astrophysical applications. Astrophys J 2014;789:2. doi:10. 1088/0004-637X/789/1/2.

[202] Nikitin AV, Rey M, Tyuterev VG. New dipole moment surfaces of methane. Chem Phys Lett 2013;565:5-11. doi:10.1016/j.cplett.2013.02.022.

[203] Nikitin AV, Rey M, Tyuterev VG. Rotational and vibrational energy levels of methane calculated from a new potential energy surface. Chem Phys Lett 2011;501:179-86. doi:10.1016/j.cplett.2010.11.008.

[204] Nikitin AV, Rey M, Tyuterev VG. First fully ab initio potential energy surface of methane with a spectroscopic accuracy. J Chem Phys 2016;145:114309. doi: $10.1063 / 1.4961973$

[205] Rey M, Nikitin A V, Babikov YL, Tyuterev VG. TheoReTS-An information system for theoretical spectra based on variational predictions from molecular potential energy and dipole moment surfaces. J Mol Spectrosc 2016;327:13858. doi:10.1016/j.jms.2016.04.006.

[206] Bray C, Cuisset A, Hindle F, Mouret G, Bocquet R, Boudon V. Spectral lines of methane measured up to $2.6 \mathrm{THz}$ at sub-MHz accuracy with a CW-THz photomixing spectrometer: Line positions of rotational transitions induced by centrifugal distortion. J Quant Spectrosc Radiat Transf 2017 This Issue. doi:10. 1016/j.jqsrt.2017.04.010.

[207] Daumont L, Nikitin AV, Thomas X, Régalia L, Von der Heyden P, Tyuterev VG, et al. New assignments in the $2 \mu \mathrm{m}$ transparency window of the ${ }^{12} \mathrm{CH}_{4}$ Octad band system. J Quant Spectrosc Radiat Transf 2013;116:101-9. doi:10.1016/j. jqsrt.2012.08.025.

[208] Hilico J-C, Robert O, Loëte M, Toumi S, Pine AS, Brown LR. Analysis of the interacting octad system of ${ }^{12} \mathrm{CH}_{4}$. J Mol Spectrosc 2001;208:1-13. doi:10.1006/ jmsp.2001.8364.

[209] Tyuterev V, Rey M, Delahaye T, Tashkun S, Nikitin A V, Kochanov R. New approach for spectroscopic data reduction using ab initio calculations and experimental lines: application to methane. 13th Bienn. HITRAN Conf.. Cambridge, MA; 2014. doi:10.5281/zenodo.11210.

[210] Nikitin AV, Boudon V, Wenger C, Albert S, Brown LR, Bauerecker S, et al. High resolution spectroscopy and the first global analysis of the Tetradecad region of methane ${ }^{12} \mathrm{CH}_{4}$. Phys Chem Chem Phys 2013;15:10071. doi:10.1039/ c3cp50799h.

[211] Nikitin AV, Lyulin OM, Mikhailenko SN, Perevalov VI, Filippov NN, Grigoriev IM, et al. GOSAT-2009 methane spectral line list in the $5550-6236 \mathrm{~cm}^{-1}$ range. J Quant Spectrosc Radiat Transf 2010;111:2211-24. doi:10.1016/j.jqsrt. 2010.05.010 
[212] Campargue A, Leshchishina O, Wang L, Mondelain D, Kassi S. The WKLMC empirical line lists $\left(5852-7919 \mathrm{~cm}^{-1}\right)$ for methane between $80 \mathrm{~K}$ and 296 K: "Final" lists for atmospheric and planetary applications. J Mol Spectrosc 2013;291:16-22. doi:10.1016/j.jms.2013.03.001.

[213] Rey M, Nikitin AV, Tyuterev VG. Complete nuclear motion Hamiltonian in the irreducible normal mode tensor operator formalism for the methane molecule. J Chem Phys 2012;136:244106, . doi:10.1063/1.4730030.

[214] Nikitin AV, Rey M, Champion JP, Tyuterev VG. Extension of the MIRS computer package for the modeling of molecular spectra: From effective to full ab initio ro-vibrational Hamiltonians in irreducible tensor form. J Quant Spectrosc Radiat Transf 2012;113:1034-42. doi:10.1016/j.jqsrt.2012.01.027.

[215] Zolot AM, Giorgetta FR, Baumann E, Swann WC, Coddington I, Newbury NR. Broad-band frequency references in the near-infrared: Accurate dual comb spectroscopy of methane and acetylene. J Quant Spectrosc Radiat Transf 2013;118:26-39. doi:10.1016/j.jqsrt.2012.11.024.

[216] Rey M, Nikitin A V, Campargue A, Kassi S, Mondelain D, Tyuterev VG. $\mathrm{Ab}$ initio variational predictions for understanding highly congested spectra: rovibrational assignment of 108 new methane sub-bands in the icosad range $\left(6280-7800 \mathrm{~cm}^{-1}\right)$. Phys Chem Chem Phys 2016;18:176-89. doi:10. 1039/С5СР05265C

[217] Nikitin AV, Rey M, Tyuterev VG. Accurate line intensities of methane from first-principles calculations. J Quant Spectrosc Radiat Transf 2017;200:90-9. doi:10.1016/j.jqsrt.2017.05.023.

[218] Brown LR. Empirical line parameters of methane from 1.1 to $2.1 \mu \mathrm{m}$. J Quant Spectrosc Radiat Transf 2005;96:251-70. doi:10.1016/j.jqsrt.2004.12.037.

[219] Campargue A, Wang L, Liu AW, Hu SM, Kassi S. Empirical line parameters of methane in the $1.63-1.48 \mu \mathrm{m}$ transparency window by high sensitivity Cavity Ring Down Spectroscopy. Chem Phys 2010;373:203-10. doi:10.1016/j. chemphys.2010.05.011

[220] Benner DC, Devi VM, O’Brien JJ, Shaji S, Spickler PT, Houck CP, et al. Empirical line parameters of $\mathrm{CH}_{4}$ from 10923 to $11502 \mathrm{~cm}^{-1}$ 2017:in preparation.

[221] Kassi S, Gao B, Romanini D, Campargue A. The near-infrared $(1.30-1.70 \mu \mathrm{m})$ absorption spectrum of methane down to $77 \mathrm{~K}$. Phys Chem Chem Phys 2008;10:4410. doi:10.1039/b805947k.

[222] Brown LR, Nikitin AV, Sung K, Rey M, Tashkun SA, Tyuterev VG, et al. Measurements and modeling of cold ${ }^{13} \mathrm{CH}_{4}$ spectra in the $3750-4700 \mathrm{~cm}^{-1}$ region. J Quant Spectrosc Radiat Transf 2016;174:88-100. doi:10.1016/j.jqsrt. 2016.01.016.

[223] Campargue A, Segovia JL, Béguier S, Kassi S, Mondelain D. The absorption spectrum of ${ }^{13} \mathrm{CH}_{4}$ in the region of the $2 v_{3}$ band at $1.66 \mu \mathrm{m}$ : Empirical line lists and temperature dependence. J Quant Spectrosc Radiat Transf 2015;152:140-8. doi:10.1016/j.jqsrt.2014.11.012.

[224] Rey M, Nikitin AV, Tyuterev VG. Predictions for methane spectra from potential energy and dipole moment surfaces: Isotopic shifts and comparative study of ${ }^{13} \mathrm{CH}_{4}$ and ${ }^{12} \mathrm{CH}_{4}$. J Mol Spectrosc 2013;291:85-97. doi:10.1016/j.jms. 2013.04.003.

[225] Campargue A, Béguier S, Zbiri Y, Mondelain D, Kassi S, Karlovets EV, et al. The ${ }^{13} \mathrm{CH}_{4}$ absorption spectrum in the Icosad range $\left(6600-7692 \mathrm{~cm}^{-1}\right)$ at $80 \mathrm{~K}$ and $296 \mathrm{~K}$ : Empirical line lists and temperature dependence. J Mol Spectrosc 2016;326:115-21. doi:10.1016/j.jms.2016.01.005

[226] Bray C, Cuisset A, Hindle F, Bocquet R, Mouret G, Drouin BJ. $\mathrm{CH}_{3}$ D photomixing spectroscopy up to 2.5THz: New set of rotational and dipole parameters, first THz self-broadening measurements. J Quant Spectrosc Radiat Transf 2017;189:198-205. doi:10.1016/j.jqsrt.2016.11.011.

[227] Delahaye T, Landsheere X, Pangui E, Huet F, Hartmann J-M, Tran H. Measurements of $\mathrm{H}_{2} \mathrm{O}$ broadening coefficients of infrared methane lines. J Quant Spectrosc Radiat Transf 2016;173:40-8. doi:10.1016/j.jqsrt.2015.12.015.

[228] Fissiaux L, Delière $\mathrm{Q}$, Blanquet $\mathrm{G}$, Robert $\mathrm{S}$, Vandaele $\mathrm{AC}$, Lepère $\mathrm{M}$. $\mathrm{CO}_{2}$ broadening coefficients in the $v_{4}$ fundamental band of methane at room temperature and application to $\mathrm{CO}_{2}$-rich planetary atmospheres. J Mol Spectrosc 2014;297:35-40. doi:10.1016/j.jms.2014.01.006.

[229] Ghysels M, Gomez L, Cousin J, Tran H, Amarouche N, Engel A, et al. Temperature dependences of air-broadening air-narrowing and line-mixing coefficients of the methane $v_{3} \mathrm{R}(6)$ manifold lines-Application to in-situ measurements of atmospheric methane. J Quant Spectrosc Radiat Transf 2014:133:206-16. doi:10.1016/j.jqsrt.2013.08.003.

[230] Jahjah M, Nguyen L, Moreno MP, Cadoret M, Zondy J-J. Measurements of $\mathrm{N}_{2}$ broadening and pressure-shift coefficients in the $\nu_{3}$-band of ${ }^{12} \mathrm{CH}_{4}$ using a cw-OPO. J Quant Spectrosc Radiat Transf 2015;151:251-9. doi:10.1016/j.jqsrt. 2014.10.006.

[231] Kapitanov VA, Ponomarev YN, Protasevich AE, Osipov KY. Lineshape models testing on $\mathrm{CH}_{4}$ spectral line $6105.6257 \mathrm{~cm}^{-1}$ (R9F1, R9F2) of $2 \nu_{3}$ band broadened by $\mathrm{N}_{2}$ and Ne. J Mol Spectrosc 2013;291:57-60. doi:10.1016/j.jms.2013. 03.009.

[232] Devi VM, Benner DC, Sung K, Brown LR, Crawford TJ, Yu S, et al. Spectral line parameters including line shapes in the $2 v_{3} \mathrm{Q}$ branch of ${ }^{12} \mathrm{CH}_{4}$. J Quant Spectrosc Radiat Transf 2016:177:152-69. doi:10.1016/j.jqsrt.2015.12.009.

[233] Predoi-Cross A, Malathy Devi V, Sutradhar P, Sinyakova T, Buldyreva J, Sung K, et al. Temperature dependences of self- and $\mathrm{N}_{2}$-broadened line-shape parameters in the $\nu_{3}$ and $\nu_{5}$ bands of ${ }^{12} \mathrm{CH}_{3} \mathrm{D}$ : Measurements and calculations. Quant Spectrosc Radiat Transf 2016;177:181-215. doi:10.1016/j.jqsrt.2016.01. 003.

[234] Tang Y, Yang SL, Lehmann KK. Measurements of $\mathrm{CH}_{3} \mathrm{D}$ line strengths, foreign pressure-broadening, and pressure-shift coefficients at near-IR region using continuous-wave cavity ring-down spectroscopy. J Mol Spectrosc 2013;291:48-56. doi:10.1016/j.jms.2013.03.004
[235] Mendonca J, Strong K, Sung K, Devi VM, Toon GC, Wunch D, et al. Using highresolution laboratory and ground-based solar spectra to assess $\mathrm{CH}_{4}$ absorption coefficient calculations. J Quant Spectrosc Radiat Transf 2017;190:48-59. doi:10.1016/j.jqsrt.2016.12.013

[236] Hill C, Gordon IE, Rothman LS, Tennyson J. A new relational database structure and online interface for the HITRAN database. J Quant Spectrosc Radiat Transf 2013;130:51-61. doi:10.1016/j.jqsrt.2013.04.027.

[237] Tran H, Flaud P-M, Fouchet T, Gabard T, Hartmann J-M. Model, software and database for line-mixing effects in the $v 3$ and $v 4$ bands of $\mathrm{CH}_{4}$ and tests using laboratory and planetary measurements-II: $\mathrm{H}_{2}$ (and $\mathrm{He}$ ) broadening and the atmospheres of Jupiter and Saturn. J Quant Spectrosc Radiat Transf 2006;101:306-24. doi:10.1016/j.jqsrt.2005.11.033.

238] Tran H, Flaud P-M, Gabard T, Hase F, von Clarmann T, Camy-Peyret C, et al. Model, software and database for line-mixing effects in the $v_{3}$ and $v_{4}$ bands of $\mathrm{CH}_{4}$ and tests using laboratory and planetary measurements-I: $\mathrm{N}_{2}$ (and air) broadenings and the earth atmosphere. J Quant Spectrosc Radiat Transf 2006;101:284-305. doi:10.1016/j.jqsrt.2005.11.020.

[239] Tran H, Hartmann J-M, Toon G, Brown LR, Frankenberg C, Warneke T, et al. The $2 v_{3}$ band of $\mathrm{CH} 4$ revisited with line mixing: Consequences for spectroscopy and atmospheric retrievals at $1.67 \mu \mathrm{m}$. J Quant Spectrosc Radiat Transf 2010;111:1344-56. doi:10.1016/j.jqsrt.2010.02.015

[240] Yu S, Drouin BJ, Miller CE. High resolution spectral analysis of oxygen. IV. Energy levels, partition sums, band constants, RKR potentials, FranckCondon factors involving the $\mathrm{X}^{3} \Sigma_{\mathrm{g}}{ }^{-}, \mathrm{a}^{1} \Delta_{\mathrm{g}}$ and $\mathrm{b}^{1} \Sigma_{\mathrm{g}}{ }^{+}$states. J Chem Phys 2014;141:174302. doi: $10.1063 / 1.4900510$.

[241] Rothman LS, Gamache RR, Goldman A, Brown LR, Toth RA, Pickett HM, et al The HITRAN database: 1986 edition. Appl Opt 1987;26:4058. doi:10.1364/AO. 26.004058 .

[242] Drouin BJ, Gupta H, Yu S, Miller CE, Müller HSP. High resolution spectral analysis of oxygen. II. Rotational spectra of a ${ }^{1} \Delta_{\mathrm{g}} \mathrm{O}_{2}$ isotopologues. J Chem Phys 2012:137:24305. doi:10.1063/1.4719169.

[243] Drouin BJ, Yu S, Elliott BM, Crawford TJ, Miller CE. High resolution spectral analysis of oxygen. III. Laboratory investigation of the airglow bands. J Chem Phys 2013;139:144301. doi:10.1063/1.4821759.

[244] Yu S, Miller CE, Drouin BJ, Müller HSP. High resolution spectral analysis of oxygen. I. Isotopically invariant Dunham fit for the $\mathrm{X}^{3} \Sigma_{\mathrm{g}}{ }^{-}, \mathrm{a}^{1} \Delta \mathrm{g}, \mathrm{b} \Sigma_{\mathrm{g}}{ }^{+}$states. J Chem Phys 2012;137:24304. doi:10.1063/1.4719170.

[245] Edwards HGM, Long DA, Najm KAB. The pure rotation Raman spectrum of ${ }^{17} \mathrm{O}^{18} \mathrm{O}$. J Raman Spectrosc 1986;17:431-2. doi:10.1002/jrs.1250170509.

[246] Leshchishina O, Kassi S, Gordon IE, Yu S, Campargue A. The band of ${ }^{16} \mathrm{O}^{17} \mathrm{O}$, ${ }^{17} \mathrm{O}^{18} \mathrm{O}$ and ${ }^{17} \mathrm{O}_{2}$ by high sensitivity CRDS near $1.27 \mu \mathrm{m}$. J Quant Spectrosc Radiat Transf 2011:112:1257-65. doi:10.1016/j.jqsrt.2011.01.014.

[247] Leshchishina O, Kassi S, Gordon IE, Rothman LS, Wang L, Campargue A. High sensitivity CRDS of the $\mathrm{a}^{1} \Delta_{\mathrm{g}}-\mathrm{X}^{3} \Sigma_{\mathrm{g}}$ - band of oxygen near $1.27 \mu \mathrm{m}$ : extended observations, quadrupole transitions, hot bands and minor isotopologues. J Quant Spectrosc Radiat Transf 2010;111:2236-45. doi:10.1016/j.jqsrt.2010.05. 014.

[248] Kassi S, Leshchishina O, Gordon IE, Yu S, Campargue A. Hyperfine structure of the transitions of ${ }^{16} \mathrm{O}^{17} \mathrm{O},{ }^{17} \mathrm{O}^{18} \mathrm{O}$ and ${ }^{17} \mathrm{O}_{2}$ by CRDS at $80 \mathrm{~K}$. Chem Phys Lett 2011;502:37-41. doi:10.1016/j.cplett.2010.12.017

[249] Gordon IE, Kassi S, Campargue A, Toon GC. First identification of the electric quadrupole transitions of oxygen in solar and laboratory spectra. J Quant Spectrosc Radiat Transf 2010;111:1174-83. doi:10.1016/j.jqsrt.2010.01.008.

[250] Long DA, Havey DK, Yu SS, Okumura M, Miller CE, Hodges JT. $\mathrm{O}_{2}$ A-band line parameters to support atmospheric remote sensing. Part II: The rare isotopologues. J Quant Spectrosc Radiat Transf 2011;112:2527-41. doi:10.1016/j.jqsrt. 2011.07.002.

[251] Long DA, Havey DK, Okumura M, Miller CE, Hodges JT. $\mathrm{O}_{2}$ A-band line parameters to support atmospheric remote sensing. J Quant Spectrosc Radiat Transf 2010;111:2021-36. doi:10.1016/j.jqsrt.2010.05.011.

[252] Drouin BJ, Benner DC, Brown LR, Cich MJ, Crawford TJ, Devi VM, et al. Multispectrum analysis of the oxygen A-band. J Quant Spectrosc Radiat Transf 2017:186:118-38. doi:10.1016/j.jqsrt.2016.03.037.

[253] Tran H, Boulet C, Hartmann J-M. Line mixing and collision-induced absorption by oxygen in the A band: laboratory measurements, model, and tools for atmospheric spectra computations. J Geophys Res 2006;111:D15210. doi:10.1029/2005JD006869.

[254] Brown L, Plymate C. Experimental Line Parameters of the Oxygen A Band at $760 \mathrm{~nm}$. J Mol Spectrosc 2000;199:166-79. doi:10.1006/jmsp.1999.8012.

[255] Tran $\mathrm{H}$, Hartmann J-M. An improved $\mathrm{O}_{2}$ A band absorption model and its consequences for retrievals of photon paths and surface pressures. J Geophys Res 2008;113:D18104. doi:10.1029/2008JD010011.

[256] Predoi-Cross A, Holladay C, Heung H, Bouanich J-P, Mellau GC, Keller R, et al. Nitrogen-broadened lineshapes in the oxygen A-band: Experimental results and theoretical calculations. J Mol Spectrosc 2008;251:159-75. doi:10.1016/j. jms.2008.02.010.

[257] Predoi-Cross A, Hambrook K, Keller R, Povey C, Schofield I, Hurtmans D, et al. Spectroscopic lineshape study of the self-perturbed oxygen A-band. J Mol Spectrosc 2008;248:85-110. doi:10.1016/j.jms.2007.11.007.

[258] Biennier L Campargue A. High resolution spectrum of the (3-0) band of the $\mathrm{b}^{1} \Sigma^{+}{ }_{g}-\mathrm{X}^{3} \Sigma^{-}{ }_{g}$ red atmospheric system of oxygen. J Mol Spectrosc 1998;188:248-50. doi:10.1006/jmsp.1997.7521.

[259] Gordon IE, Rothman LS, Toon GC. Revision of spectral parameters for the Band $\gamma$-bands of oxygen and their validation against atmospheric spectra. J Quant Spectrosc Radiat Transf 2011;112:2310-22. doi:10.1016/j.jqsrt.2011.05. 007 
[260] Domysławska J, Wójtewicz S, Masłowski P, Cygan A, Bielska K, Trawiński RS, et al. A new approach to spectral line shapes of the weak oxygen transitions for atmospheric applications. J Quant Spectrosc Radiat Transf 2016;169:11121. doi:10.1016/j.jqsit.2015.10.019.

[261] Domysławska J, Wójtewicz S, Masłowski P, Cygan A, Bielska K, Trawiński RS, et al. Spectral line shapes and frequencies of the molecular oxygen B-band R-branch transitions. J Quant Spectrosc Radiat Transf 2015;155:22-31. doi:10. 1016/j.jqsrt.2014.12.015

[262] Wójtewicz S, Cygan A, Masłowski P, Domysławska J, Lisak D, Trawiński RS, et al. Spectral line shapes of self-broadened P-branch transitions of oxygen B band. J Quant Spectrosc Radiat Transf 2014;144:36-48. doi:10.1016/j.jqsrt. 2014.03.029

[263] Müller HSP, Kobayashi K, Takahashi K, Tomaru K, Matsushima F. Terahertz spectroscopy of $\mathrm{N}^{18} \mathrm{O}$ and isotopic invariant fit of several nitric oxide isotopologs. J Mol Spectrosc 2015;310:92-8. doi:10.1016/j.jms.2014.12.002.

[264] Pickett HM, Cohen EA, Waters JW, Phillips T. The rotational spectrum of NO. In: Abstr. OSU Int. Symp. Mol. Spectrosc; 1979. p. 1970-9.

[265] Varberg TD, Stroh F, Evenson KM. Far-infrared rotational and fine-structure transition frequencies and molecular constants of ${ }^{14} \mathrm{NO}$ and ${ }^{15} \mathrm{NO}$. J Mol Spectrosc 1999;196:5-13. doi:10.1006/jmsp.1999.7850.

[266] Meerts WL, Dymanus A. The hyperfine $\Lambda$-doubling spectrum of ${ }^{14} \mathrm{~N}^{16} \mathrm{O}$ and ${ }^{14} \mathrm{~N}^{16}$ O. J Mol Spectrosc 1972;44:320-46. doi:10.1016/0022-2852(72)90109-9.

[267] Meerts WL. A theoretical reinvestigation of the rotational and hyperfine lambda doubling spectra of diatomic molecules with a ${ }^{2} \Pi$ state: the spectrum of NO. Chem Phys 1976;14:421-5. doi:10.1016/0301-0104(76)80139-5.

[268] Neumann RM. High-precision radiofrequency spectrum of ${ }^{14} \mathrm{~N}^{16} \mathrm{O}$. Astrophys J 1970;161:779. doi:10.1086/150578.

[269] Dale RM, Johns JWC, McKellar ARW, Riggin M. High-resolution laser magnetic resonance and infrared-radiofrequency double-resonance spectroscopy of NO and its isotopes near 5.4 $\mu \mathrm{m}$. J Mol Spectrosc 1977;67:440-58. doi:10.1016/ 0022-2852(77)90051-0.

[270] Lowe RS, McKellar ARW, Veillette P, Meerts WL. Hyperfine and $\Lambda$-doubling parameters for the $\mathrm{v}=1$ state of NO from infrared-radiofrequency double resonance. J Mol Spectrosc 1981;88:372-7. doi:10.1016/0022-2852(81)90187-9.

[271] Hinz A, Wells JS, Maki AG. Heterodyne frequency measurements on the nitric oxide fundamental band. J Mol Spectrosc 1986;119:120-5. doi:10.1016/ 0022-2852(86)90206-7.

[272] Saupe S, Meyer B, Wappelhorst MH, Urban W, Maki AG. Sub-doppler heterodyne frequency measurements and calibration tables for the nitric oxide fundamental band. J Mol Spectrosc 1996;179:13-21. doi:10.1006/jmsp.1996.0179.

[273] Saleck AH, Yamada KMT, Winnewisser G. Isotopic nitric oxide spectra and breakdown of the Born-Oppenheimer approximation. Mol Phys 1991;72:1135-48. doi:10.1080/00268979100100811.

[274] Saleck AH, Liedtke M, Dolgner A, Winnewisser G. Rotational spectra of ${ }^{14} \mathrm{~N}^{17} \mathrm{O}$ and ${ }^{15} \mathrm{~N}^{18} \mathrm{O}$. Zeitschrift Für Naturforsch A 1994;49. doi:10.1515/ zna-1994-1203.

[275] Liu Y, Guo Y, Lin J, Huang G, Duan C, Li F. Measurement of the electric dipole moment of $\mathrm{NO}\left(\mathrm{X}^{2} \Pi v=0,1\right)$ by mid-infrared laser magnetic resonance spectroscopy. Mol Phys 2001;99:1457-61. doi:10.1080/00268970110059642.

[276] Curl RF. The relationship between electron spin rotation coupling constants and g -tensor components. Mol Phys 1965;9:585-97. doi:10.1080/ 00268976500100761 .

[277] Wong A, Yurchenko SN, Bernath P, Müller HSP, McConkey S, Tennyson J. ExoMol line list XXI: nitric oxide (NO). Mon Not R Astron Soc 2017;870:882-97. doi:10.1093/mnras/stx1211.

[278] Rothman LS, Gordon IE, Barber RJ, Dothe H, Gamache RR, Goldman a, et al. HITEMP, the high-temperature molecular spectroscopic database. J Quant Spectrosc Radiat Transf 2010;111:2139-50. doi:10.1016/j.jqsrt.2010.05.001.

[279] Semenov M, Yurchenko SN, Tennyson J. Predicted Landé g-factors for open shell diatomic molecules. J Mol Spectrosc 2016;330:57-62. doi:10.1016/j.jms. 2016.11.004

[280] Cazzoli G, Puzzarini C. $\mathrm{N}_{2-}, \mathrm{O}_{2-}, \mathrm{H}_{2-}$, and He-broadening of $\mathrm{SO}_{2}$ rotational lines in the mm-/submm-wave and $\mathrm{THz}$ frequency regions: The $\mathrm{J}$ and $\mathrm{Ka}$ dependence. J Quant Spectrosc Radiat Transf 2012;113:1051-7. doi:10.1016/ j.jqsit.2012.01.011.

[281] Tasinato N, Pietropolli Charmet A, Stoppa P, Giorgianni S, Buffa G. $\mathrm{N}_{2-}, \mathrm{O}_{2}-$ and He-collision-induced broadening of sulfur dioxide ro-vibrational lines in the $9.2 \mu \mathrm{m}$ atmospheric window. Spectrochim Acta-Part A Mol Biomol Spectrosc 2014;118:373-9. doi:10.1016/j.saa.2013.08.071

[282] Kühnemann F, Heiner Y, Sumpf B, Herrmann K. Line broadening in the $\nu_{3}$ band of $\mathrm{SO}_{2}$ : studied with diode laser spectroscopy. J Mol Spectrosc 1992;152:1-12. doi:10.1016/0022-2852(92)90111-Z.

[283] Sumpf B, Fleischmann O, Kronfeldt H-D. Self-, air-, and nitrogen-broadening in the $v_{1}$ band of $\mathrm{SO}_{2}$. J Mol Spectrosc 1996;176:127-32. doi:10.1006/jmsp. 1996.0068

[284] Ball CD, Dutta JM, Goyette TM, Helminger P, De Lucia FC. The pressure broadening of $\mathrm{SO}_{2}$ by $\mathrm{N}_{2}, \mathrm{O}_{2}$, He, and $\mathrm{H}_{2}$ between 90 and $500 \mathrm{~K}$. J Quant Spectrosc Radiat Transf 1996;56:109-17. doi:10.1016/0022-4073(96)00016-7.

[285] Henningsen J, Barbe A, De Backer-Barilly M-R. Revised molecular parameters for ${ }^{32} \mathrm{~S}^{16} \mathrm{O}_{2}$ and ${ }^{34} \mathrm{~S}^{16} \mathrm{O}_{2}$ from high resolution study of the infrared spectrum in the 7-8 $\mu \mathrm{m}$ wavelength region. J Quant Spectrosc Radiat Transf 2008;109:2491-510. doi:10.1016/j.jqsrt.2008.04.001.

[286] Tan Y, Kochanov RV, Rothman LS, Gordon IE. The broadening coefficients of $\mathrm{SO}_{2}$. 2016. doi:10.5281/zenodo.163447.

[287] Tasinato N, Charmet AP, Stoppa P, Buffa G, Puzzarini C. A complete listing of sulfur dioxide self-broadening coefficients for atmospheric applications by coupling infrared and microwave spectroscopy to semiclassical calculations. J Quant Spectrosc Radiat Transf 2013;130:233-48. doi:10.1016/j.jqsrt.2013.03. 015 .

[288] Sumpf B, Fleischmann O, Waschull J, Heiner Y, Kronfeldt H-D. Noble gas pressure broadening in the $\mathrm{v}_{1}$ and $\mathrm{v}_{3}$ band of $\mathrm{SO}_{2}$ studied with IR tunable diode laser spectroscopy. Infrared Phys Technol 1995;36:439-45. doi:10.1016/ 1350-4495(94)00084-X

[289] Krishnaji, Chandra S. Molecular interaction and linewidth of the asymmetric molecule $\mathrm{SO}_{2}$. II. $\mathrm{SO}_{2}-\mathrm{CO}_{2}$ collisions. J Chem Phys 1963;38:1019. doi:10.1063/ 1.1733747.

[290] Huang X, Schwenke DW, Lee TJ. Ames ${ }^{32} \mathrm{~S}^{16} \mathrm{O}^{18} \mathrm{O}$ line list for high-resolution experimental IR analysis. J Mol Spectrosc 2016;330:101-11. doi:10.1016/j.jms. 2016.08.013.

[291] Huang X, Schwenke DW, Lee TJ. Empirical infrared line lists for five $\mathrm{SO}_{2}$ isotopologues: 32/33/34/36S16O2 and 32S1802. J Mol Spectrosc 2015;311:19-24. doi:10.1016/j.jms.2015.01.010.

[292] Underwood DS, Tennyson J, Yurchenko SN, Huang X, Schwenke DW, Lee TJ, et al. ExoMol molecular line lists-XIV. The rotation-vibration spectrum of hot $\mathrm{SO}_{2}$. Mon Not R Astron Soc 2016;459:3890-9. doi:10.1093/mnras/stw849.

[293] Ulenikov ON, Bekhtereva ES, Gromova OV, Zamotaeva VA, Sklyarova EA, Sydow $C$, et al. First high resolution analysis of the $2 v_{1}, 2 v_{3}$, and $v_{1}+v_{3}$ bands of $\mathrm{S}^{18} \mathrm{O}_{2}$. J Quant Spectrosc Radiat Transf 2016;185:12-21. doi:10.1016/ j.jqsrt.2016.08.008.

[294] Ulenikov ON, Gromova OV, Bekhtereva ES, Krivchikova YV, Sklyarova EA, Buttersack T, et al. High resolution FTIR study of ${ }^{34} \mathrm{~S}^{16} \mathrm{O}_{2}$ : the bands $2 v_{3}, 2 v_{1}+v_{2}$ and $2 v_{1}+v_{2}-v_{2}$. J Mol Spectrosc 2015;318:26-33. doi:10.1016/j.jms.2015.09. 009 .

[295] Ulenikov ON, Gromova OV, Bekhtereva ES, Bolotova IB, Konov IA, Horneman V-M, et al. High resolution analysis of the $\mathrm{SO}_{2}$ spectrum in the 2600$2900 \mathrm{~cm}^{-1}$ region: $2 v_{3}, v_{2}+2 v_{3}-v_{2}$ and $2 v_{1}+v_{2}$ bands. J Quant Spectrosc Radiat Transf 2012;113:500-17. doi:10.1016/j.jqsrt.2012.01.006.

[296] Ulenikov ON, Bekhtereva ES, Gromova OV, Zamotaeva VA, Kuznetsov SI, Sydow C, et al. High resolution study of the rotational structure of doubly excited vibrational states of ${ }^{32} \mathrm{~S}^{16} \mathrm{O}^{18} \mathrm{O}$ : the first analysis of the $2 v_{1}$ $\nu_{1}+v_{3}$, and $2 v_{3}$ bands. J Quant Spectrosc Radiat Transf 2017;189:344-50. doi:10.1016/j.jqsrt.2016.12.019.

[297] Ulenikov ON, Gromova OV, Bekhtereva ES, Fomchenko AL, Sydow C, Bauerecker S. First high resolution analysis of the $3 v_{1}$ band of ${ }^{34} \mathrm{~S}^{16} \mathrm{O}_{2}$. J Mol Spectrosc 2016;319:50-4. doi:10.1016/j.jms.2015.11.002.

[298] Belov SP, Klaus T, Plummer GM, Schieder R, Winnewisser G. Sub-doppler spectroscopy of ammonia near $570 \mathrm{GHz}$. Zeitschrift Für Naturforsch A 1995;50. doi:10.1515/zna-1995-1224.

[299] Winnewisser G, Belov SP, Klaus T, Urban S. Ro-inversional spectrum of ammonia. Zeitschrift Für Naturforsch A 1996;51. doi:10.1515/zna-1996-0312.

[300] Kukolich SG. Hyperfine Structure of ${ }^{15} \mathrm{NH}_{3}$. Phys Rev 1968;172:59-63. doi:10. 1103/PhysRev.172.59.

[301] Hougen JT. Reinterpretation of molecular beam hyperfine data for ${ }^{14} \mathrm{NH}_{3}$ and ${ }^{15} \mathrm{NH}_{3}$. J Chem Phys 1972;57:4207-17. doi:10.1063/1.1678050.

[302] Sasada H. Microwave inversion spectrum of ${ }^{15} \mathrm{NH}_{3}$. J Mol Spectrosc 1980;83:15-20. doi:10.1016/0022-2852(80)90307-0.

[303] Urban S, Klee S, Yamada KMT. Ground state ro-inversional transitions of ${ }^{15} \mathrm{NH}_{3}$ in the far-infrared region. J Mol Spectrosc 1994;168:384-9. doi:10. 1006/jmsp.1994.1287.

[304] Carlotti M, Trombetti A, Velino B, Vrbancich J. The rotation-inversion spectrum of ${ }^{15} \mathrm{NH}_{3}$. J Mol Spectrosc 1980;83:401-7. doi:10.1016/0022-2852(80) 90064-8.

[305] Chen P, Pearson JC, Pickett HM, Matsuura S, Blake GA. Measurements of ${ }^{14} \mathrm{NH}_{3}$ in the $v_{2}=1$ state by a solid-state, photomixing, $\mathrm{THz}$ spectrometer, and a simultaneous analysis of the microwave, terahertz, and infrared transitions between the ground and $v_{2}$ inversion-rotation levels. J Mol Spectrosc 2006;236:116-26. doi:10.1016/j.jms.2006.01.002.

[306] Huang X, Schwenke DW, Lee TJ. Rovibrational spectra of ammonia. II. Detailed analysis, comparison, and prediction of spectroscopic assignments for ${ }^{14} \mathrm{NH}_{3},{ }^{15} \mathrm{NH}_{3}$, and ${ }^{14} \mathrm{ND}_{3}$. J Chem Phys 2011;134:44321. doi:10.1063/1. 3541352 .

[307] Tanaka K, Ito $\mathrm{H}$, Tanaka $\mathrm{T}$. $\mathrm{CO}_{2}$ laser-microwave double resonance spectroscopy of $\mathrm{NH}_{3}$ : precise measurement of dipole moment in the ground state. J Chem Phys 1987;87:1557-67. doi:10.1063/1.453725.

[308] Barton EJ, Yurchenko SN, Tennyson J, B?guier S, Campargue A. A near infrared line list for $\mathrm{NH}_{3}$ : analysis of a Kitt Peak spectrum after 35 years. J Mol Spectrosc 2016;325:7-12. doi:10.1016/j.jms.2016.05.001

[309] Down MJ, Hill C, Yurchenko SN, Tennyson J, Brown LR, Kleiner I. Re-analysis of ammonia spectra: updating the HITRAN ${ }^{14} \mathrm{NH}_{3}$ database. J Quant Spectrosc Radiat Transf 2013;130:260-72. doi:10.1016/j.jqsrt.2013.05.027.

[310] Barton EJ, Polyansky OL, Yurchenko SN, Tennyson J, Civiš S, Ferus M, et al. Absorption spectra of ammonia near $1 \mu \mathrm{m}$. J Quant Spectrosc Radiat Transf 2017 This issue. doi:10.1016/j.jqsrt.2017.03.042.

[311] Yurchenko SN, Barber RJ, Yachmenev A, Thiel W, Jensen P, Tennyson J. A variationally computed $\mathrm{T}=300 \mathrm{~K}$ line list for $\mathrm{NH}_{3}$. J Phys Chem A 2009;113:11845-55. doi:10.1021/jp9029425.

[312] Yurchenko SN, Barber RJ, Tennyson J. A variationally computed line list for hot $\mathrm{NH}_{3}$. Mon Not R Astron Soc 2011;413:1828-34. doi:10.1111/j.1365-2966. 2011.18261.x.

[313] Al Derzi AR, Furtenbacher T, Tennyson J, Yurchenko SN, Császár AG. MARVEL analysis of the measured high-resolution spectra of ${ }^{14} \mathrm{NH}_{3}$. J Quant Spectrosc Radiat Transf 2015;161:117-30. doi:10.1016/j.jqsrt.2015.03.034. 
[314] Sharpe SW, Johnson TJ, Sams RL, Chu PM, Rhoderick GC, Johnson PA. Gas-phase databases for quantitative infrared spectroscopy. Appl Spectrosc 2004;58:1452-61. doi:10.1366/0003702042641281.

[315] Polyansky OL, Ovsyannikov RI, Kyuberis AA, Lodi L, Tennyson J, Yachmenev A, et al. Calculation of rotation-vibration energy levels of the ammonia molecule based on an ab initio potential energy surface. J Mol Spectrosc 2016;327:2130. doi:10.1016/j.jms.2016.08.003.

[316] Nemtchinov V, Sung K, Varanasi P. Measurements of line intensities and half-widths in the $10-\mu \mathrm{m}$ bands of ${ }^{14} \mathrm{NH}_{3}$. J Quant Spectrosc Radiat Transf 2004;83:243-65. doi:10.1016/S0022-4073(02)00354-0.

[317] Baldacchini G, D'Amato F, Buffa G, Tarrini O, Rosa MD, Pelagalli F. Temperature dependence of foreign gas broadening and shift of the $\mathrm{aQ}(9,9)$ transition line of ammonia. J Quant Spectrosc Radiat Transf 2001;68:625-33. doi:10.1016/S0022-4073(00)00043-1.

[318] Dhib M, Echargui MA, Aroui H, Orphal J, Hartmann JM. Line shift and mixing in the $\mathrm{v}_{4}$ and $2\left(\mathrm{~V}_{2}\right)$ band of $\mathrm{NH}_{3}$ perturbed by $\mathrm{H}_{2}$ and $\mathrm{Ar}$. $\mathrm{J}$ Mol SpectrosC 2005;233:138-48. doi:10.1016/j.jms.2005.06.012.

[319] Raynaud F, Lemoine B, Rohart F. High precision pressure-induced lineshifts measured with a frequency-stabilized diode laser: application to the $v_{2}$ and $\left(2 v_{2}-v_{2}\right)$ bands of $\mathrm{NH}_{3}$. J Mol Spectrosc 1994;168:584-92. doi:10.1006/jmsp. 1994.1304.

[320] Pine AS, Markov VN, Buffa G, Tarrini O. $\mathrm{N}_{2}, \mathrm{O}_{2}, \mathrm{H}_{2}, \mathrm{Ar}$ and He broadening in the $v_{1}$ band of $\mathrm{NH}_{3}$. J Quant Spectrosc Radiat Transf 1993;50:337-48. doi:10. 1016/0022-4073(93)90069-T.

[321] Dhib M, Echargui MA, Aroui H, Orphal J. Shifting and line mixing parameters in the $v_{4}$ band of $\mathrm{NH}_{3}$ perturbed by $\mathrm{CO}_{2}$ and $\mathrm{He}$ : experimental results and theoretical calculations. J Mol Spectrosc 2006;238:168-77. doi:10.1016/j.jms. 2006.04.013

[322] Broquier M, Picard-Bersellini a, Aroui H, Billing GD. Pressure broadening and cross relaxation of ammonia perturbed by hydrogen and helium: Implications on intermolecular potentials and discussion of rotational effects. J Chem Phys 1988;88:1551. doi:10.1063/1.454134.

[323] Broquier M, Picard-Bersellini A, Hall J. An inelastic rotational transfer study of $\mathrm{NH}_{3}$, colliding with $\mathrm{H}_{2}$, and He. Chem Phys Lett 1987;136:531-4. doi:10. 1016/0009-2614(87)80512-2.

[324] Keffer CE, Conner CP, Smith WH. Hydrogen broadening of vibrationalrotational transitions of ammonia lying near $6450 \AA$ A. J Quant Spectrosc Radiat Transf 1985;33:193-6. doi:10.1016/0022-4073(85)90148-7.

[325] Varanasi P. Infrared line widths at planetary atmospheric temperatures, J Quant Spectrosc Radiat Transf 1988;39:13-25. http://dx.doi.org/10.1016/ 0022-4073(88)90015-5.

[326] Baldacchini G, Bizzarri A, Nencini L, Sorge V, Buffa G, Tarrini O. Foreign-gas pressure broadening and shift of ammonia transition lines in the $v_{2}$ vibrational bands. J Quant Spectrosc Radiat Transf 1990;43:371-80. doi:10.1016/ 0022-4073(90)90003-0.

[327] Aroui H, Picard-Bersellini A, Chevalier M, Broquier M, Gherissi S. Pressurebroadening and cross-relaxation rates of rotation-inversion transitions in the $v_{4}$ band of $\mathrm{NH}_{3}$ perturbed by $\mathrm{CO}_{2}$. J Mol Spectrosc 1996;176:162-8. doi:10. 1006/jmsp.1996.0073.

[328] Raspollini P, Belotti C, Burgess A, Carli B, Carlotti M, Ceccherini S, et al. MIPAS level 2 operational analysis. Atmos Chem Phys 2006;6:5605-30. doi:10.5194/ acp-6-5605-2006.

[329] Raspollini P, Carli B, Carlotti M, Ceccherini S, Dehn A, Dinelli BM, et al. Ten years of MIPAS measurements with ESA Level 2 processor V6; Part 1: Retrieval algorithm and diagnostics of the products. Atmos Meas Tech 2013;6:2419-39. doi:10.5194/amt-6-2419-2013.

[330] Wespes C, Hurtmans D, Clerbaux C, Santee ML, Martin R V, Coheur PF. Global distributions of nitric acid from IASI/MetOP measurements. Atmos Chem Phys 2009;9:7949-62. doi:10.5194/acp-9-7949-2009.

[331] Wang DY, Höpfner M, Blom CE, Ward WE, Fischer H, Blumenstock T, et al. Validation of MIPAS $\mathrm{HNO}_{3}$ operational data. Atmos Chem Phys 2007;7:490534. doi:10.5194/acp-7-4905-2007.

[332] Wolff MA, Kerzenmacher T, Strong K, Walker KA, Toohey M, Dupuy E, et al. Validation of $\mathrm{HNO}_{3}, \mathrm{ClONO}_{2}$, and $\mathrm{N}_{2} \mathrm{O}_{5}$ from the Atmospheric Chemistry Experiment Fourier Transform Spectrometer (ACE-FTS). Atmos Chem Phys 2008:8:3529-62. doi:10.5194/acp-8-3529-2008.

[333] Irie H, Sugita T, Nakajima H, Yokota T, Oelhaf H, Wetzel G, et al. Validation of stratospheric nitric acid profiles observed by Improved Limb Atmospheric Spectrometer (ILAS)-II. J Geophys Res 2006 111:D11S03. doi:10.1029/ 2005JD006115.

[334] Perrin A, Lado-Bordowsky O, Valentin A. The $\mathrm{v}_{3}$ and $\mathrm{v}_{4}$ interacting bands of $\mathrm{HNO}_{3}$ line positions and line intensities. Mol Phys 1989;67:249-70. doi:10. 1080/00268978900101061.

[335] Perrin A. New Analysis of the $\nu_{3}$ and $\nu_{4}$ Bands of $\mathrm{HNO}_{3}$ in the $7.6 \mu \mathrm{m}$ Region. J Phys Chem A 2013;117:13236-48. doi:10.1021/jp401979v.

[336] Perrin A, Flaud J-M, Ridolfi M, Vander Auwera J, Carlotti M. MIPAS database: new $\mathrm{HNO}_{3}$ line parameters at $7.6 \mu \mathrm{m}$ validated with MIPAS satellite measurements. Atmos Meas Tech 2016;9:2067-76. doi:10.5194/amt-9-2067-2016.

[337] Pavlyuchko AI, Yurchenko SN, Tennyson J. ExoMol molecular line lists-XI. The spectrum of nitric acid. Mon Not R Astron Soc 2015;452:1702-6. doi:10.1093/ mnras/stv1376.

[338] Pavlyuchko AI, Yurchenko SN, Tennyson J. A hybrid variational-perturbation calculation of the ro-vibrational spectrum of nitric acid. J Chem Phys 2015;142:94309. doi:10.1063/1.4913741.

[339] Brooke JSA, Bernath PF, Western CM, Sneden C, Afşar M, Li G, et al. Line strengths of rovibrational and rotational transitions in the $X^{2} \Pi$ ground state of OH. J Quant Spectrosc Radiat Transf 2016;168:142-57. doi:10.1016/j.jqsrt. 2015.07.021.

340] Medvedev ES, Meshkov VV, Stolyarov AV, Gordon IE. Peculiarities of highovertone transition probabilities in carbon monoxide revealed by highprecision calculation. J Chem Phys 2015;143:154301. doi:10.1063/1.4933136.

[341] Medvedev ES, Meshkov VV, Stolyarov AV, Ushakov VG, Gordon IE. Impact of the dipole-moment representation on the intensity of high overtones. J Mol Spectrosc 2016;330:36-42. doi:10.1016/j.jms.2016.06.013.

[342] Meredith RE. Broadening of hydrogen fluoride lines by $\mathrm{H}_{2}, \mathrm{D}_{2}$, and $\mathrm{N}_{2}$. J Chem Phys 1974:60:3388, doi:10.1063/1.1681547.

[343] Shaw BM, Lovell RJ. Foreign-gas broadening of $\mathrm{HF}$ by $\mathrm{CO}_{2}$. J Opt Soc Am 1969;59:1598. doi:10.1364/JOSA.59.001598.

[344] Varanasi P, Sarangi SK, Tejwani GDT. Line shape parameters for $\mathrm{HCl}$ and $\mathrm{HF}$ in a $\mathrm{CO}_{2}$ atmosphere. J Quant Spectrosc Radiat Transf 1972;12:857-72. doi:10. 1016/0022-4073(72)90074-X

[345] Guelachvili G, Smith MAH. Measurements of pressure-induced shifts in the 1-0 and 2-0 bands of $\mathrm{HF}$ and in the 2-0 bands of $\mathrm{H}^{35} \mathrm{Cl}$ and $\mathrm{H}^{37} \mathrm{Cl}$. J Quant Spectrosc Radiat Transf 1978;20:35-47. doi:10.1016/0022-4073(78)90004-3.

[346] Chou S, Baer DS, Hanson RK. Diode-laser measurements of He-, Ar-, and $\mathrm{N}_{2}$-broadened HF lineshapes in the first overtone band. J Mol Spectrosc 1999;196:70-6. doi:10.1006/jmsp.1999.7847.

[347] Bonczyk PA. Determination of the linewidth dependence on foreign-gas pressure for 3.4- $\mu \mathrm{m}$ DF spectra. Phys Rev A 1976;13:251-2. doi:10.1103/PhysRevA 13.251.

[348] Bachet G. Experimental study on elongation of first 5 lines of pure rotation of hydrogen-fluoride perturbed by strange compressed gases. Comptes Rendus Hebd Des Seances L'Academie Des Sci Ser B 1972;274:1319-21.

[349] Tudorie M, Földes T, Vandaele AC, Vander Auwera J. $\mathrm{CO}_{2}$ pressure broadening and shift coefficients for the 1-0 band of $\mathrm{HCl}$ and DCl. J Quant Spectrosc Radiat Transf 2012;113:1092-101. doi:10.1016/j.jqsrt.2012.01.025.

[350] Rank DH, Eastman DP, Rao BS, Wiggins TA. Breadths and shifts of molecular band lines due to perturbation by foreign gases. J Mol Spectrosc 1963;10:3450. doi:10.1016/0022-2852(63)90152-8

351] Hurtmans D, Henry A, Valentin A, Boulet C. Narrowing broadening and shifting parameters for $\mathrm{R}(2)$ and $\mathrm{P}(14)$ lines in the $\mathrm{HCl}$ fundamental band perturbed by $\mathrm{N}_{2}$ and rare gases from tunable diode laser spectroscopy. J Mol Spectrosc 2009;254:126-36. doi:10.1016/j.jms.2009.01.015.

[352] Rank DH, Birtley WB, Eastman DP, Wiggins TA. Pressure-Induced Shifts of $\mathrm{HCl}$ Lines Due to Foreign Gases. J Chem Phys 1960;32:296-7. doi:10.1063/ 1.1700921 .

[353] Houdeau JP, Larvor M, Haeusler C. Etude à basse température des largeurs et des déplacements des raies rovibrationnelles de la bande fondamentale de $\mathrm{H}^{35} \mathrm{Cl}$ comprimé par $\mathrm{N}_{2}, \mathrm{O}_{2}, \mathrm{D}_{2}$ et $\mathrm{H}_{2}$. Can J Phys 1980;58:318-24. doi:10. 1139/p80-046.

354] Babrov H, Ameer G, Benesch W. Molecular collision cross sections from in frared absorption measurements. J Chem Phys 1960;33:145. doi:10.1063/1 1731069 .

[355] Toth RA, Hunt RH, Plyler EK. Linewidths of $\mathrm{HCl}$ broadened by $\mathrm{H}_{2}, \mathrm{D}_{2}$, and HCN. J Chem Phys 1970;53:4303-7. doi:10.1063/1.1673937.

[356] Lejeune B, Mahieu E, Vollmer MK, Reimann S, Bernath PF, Boone CD, et al Optimized approach to retrieve information on atmospheric carbonyl sulfide (OCS) above the Jungfraujoch station and change in its abundance since 1995 J Quant Spectrosc Radiat Transf 2017;186:81-95. doi:10.1016/j.jqsrt.2016.06. 001 .

[357] Krasnopolsky VA. High-resolution spectroscopy of Venus: Detection of OCS upper limit to $\mathrm{H}_{2} \mathrm{~S}$, and latitudinal variations of $\mathrm{CO}$ and $\mathrm{HF}$ in the upper cloud layer. Icarus 2008;197:377-85. doi:10.1016/j.icarus.2008.05.020.

[358] Phillips NG, Ackley R, Crosson ER, Down A, Hutyra LR, Brondfield M, et al. Mapping urban pipeline leaks: methane leaks across Boston. Environ Pollut 2013;173:1-4. doi:10.1016/j.envpol.2012.11.003.

[359] Golebiowski D, de Ghellinck d'Elseghem Vaernewijck X, Herman M, Vander Auwera J, Fayt A. High sensitivity (femto-FT-CEAS) spectra of carbonyl sulphide between 6200 and $8200 \mathrm{~cm}^{-1}$ and new energy pattern in the global rovibrational analysis of ${ }^{16} \mathrm{O}^{12} \mathrm{C}^{32} \mathrm{~S}$. J Quant Spectrosc Radiat Transf 2014;149:184-203. doi:10.1016/j.jqsrt.2014.07.005.

[360] Rbaihi E, Belafhal A, Vander Auwera J, Naïm S, Fayt A. Fourier transform spectroscopy of carbonyl sulfide from 4800 to $8000 \mathrm{~cm}^{-1}$ and new global analysis of ${ }^{16} \mathrm{O}^{12} \mathrm{C}^{32} \mathrm{~S}$. J Mol Spectrosc 1998;191:32-44. doi:10.1006/jmsp.1998.7616.

[361] Koshelev MA, Tretyakov MY. Collisional broadening and shifting of OCS rotational spectrum lines. J Quant Spectrosc Radiat Transf 2009;110:118-28. doi:10.1016/j.jqsrt.2008.09.010.

[362] Broquier M, Picard-Bersellini A, Whitaker BJ, Green S. Rotational inelastic cross sections for OCS-Ar, OCS-He, OCS- $\mathrm{H}_{2}$ collisions: A comparison between theory and experiment. J Chem Phys 1986;84:2104-7. doi:10.1063 1.450421

[363] Liuima FA, Bushkovitch AV, Rouse AG. Pressure Broadening of OCS in Foreign Gas Mixtures. Phys Rev 1954;96:434-5. doi:10.1103/PhysRev.96.434.

[364] Mäder H, Ekkers J, Hoke W, Flygare WH. A $\pi, \tau, \pi / 2$ type pulse sequence method for the determination of $\mathrm{T} 1$ in rotational transitions. J Chem Phys 1975;62:4380-7. doi:10.1063/1.430338.

[365] Murphy JS. Collision broadening of rotational absorption lines. III. Broadening by linear molecules and inert gases and the determination of molecular quadrupole moments. J Chem Phys 1968;49:3333. doi:10.1063/1.1670605.

[366] Bouanich J-P, Campers C, Blanquet G, Walrand J. Diode-laser measurements of Ar- and $\mathrm{CO}_{2}$-broadened linewidths in the $\mathrm{v}_{1}$ band of OCS. J Quant Spectrosc Radiat Transf 1988;39:353-65. doi:10.1016/0022-4073(88)90100-8. 
[367] Vander Auwera J, Kleffmann J, Flaud J-M, Pawelke G, Bürger H, Hurtmans D, et al. Absolute $v_{2}$ line intensities of $\mathrm{HOCl}$ by simultaneous measurements in the infrared with a tunable diode laser and far-infrared region using a Fourier transform spectrometer. J Mol Spectrosc 2000;204:36-47. doi:10.1006/jmsp. 2000.8197

[368] Lutsch E, Dammers E, Conway S, Strong K. Long-range transport of $\mathrm{NH}_{3}$, $\mathrm{CO}, \mathrm{HCN}$, and $\mathrm{C}_{2} \mathrm{H}_{6}$ from the 2014 Canadian wildfires. Geophys Res Lett 2016;43:8286-97. doi:10.1002/2016GL070114.

[369] Viatte C, Strong K, Paton-Walsh C, Mendonca J, O'Neill NT, Drummond JR. Measurements of $\mathrm{CO}, \mathrm{HCN}$, and $\mathrm{C}_{2} \mathrm{H}_{6}$ total columns in smoke plumes transported from the 2010 Russian Boreal Forest Fires to the Canadian high arctic. Atmos-Ocean 2013;51:522-31. doi:10.1080/07055900.2013.823373.

[370] Akagi SK, Yokelson RJ, Wiedinmyer C, Alvarado MJ, Reid JS, Karl T, et al. Emission factors for open and domestic biomass burning for use in atmospheric models. Atmos Chem Phys 2011;11:4039-72. doi:10.5194/acp-11-4039-2011.

[371] Vigouroux C, Stavrakou T, Whaley C, Dils B, Duflot V, Hermans C, et al. FTIR time-series of biomass burning products $\left(\mathrm{HCN}, \mathrm{C}_{2} \mathrm{H}_{6}, \mathrm{C}_{2} \mathrm{H}_{2}, \mathrm{CH}_{3} \mathrm{OH}\right.$, and $\mathrm{HCOOH})$ at Reunion Island $\left(21^{\circ} \mathrm{S}, 55^{\circ} \mathrm{E}\right)$ and comparisons with model data. Atmos Chem Phys 2012;12:10367-85. doi:10.5194/acp-12-10367-2012.

[372] Viatte C, Strong K, Walker KA, Drummond JR. Five years of $\mathrm{CO}, \mathrm{HCN}, \mathrm{C}_{2} \mathrm{H}_{6}$, $\mathrm{C}_{2} \mathrm{H}_{2}, \mathrm{CH}_{3} \mathrm{OH}, \mathrm{HCOOH}$ and $\mathrm{H}_{2} \mathrm{CO}$ total columns measured in the Canadian high Arctic. Atmos Meas Tech 2014;7:1547-70. doi:10.5194/amt-7-1547-2014.

[373] Marchenko D, Neerincx AH, Mandon J, Zhang J, Boerkamp M, Mink J, et al. A compact laser-based spectrometer for detection of $\mathrm{C}_{2} \mathrm{H}_{2}$ in exhaled breath and HCN in vitro. Appl Phys B 2015;118:275-80. doi:10.1007| s00340-014-5983-y

[374] Tsiaras A, Rocchetto M, Waldmann IP, Venot O, Varley R, Morello G, et al. Detection of an Atmosphere Around The Super-Earth 55 Cancri e. Astrophys J 2016;820:99. doi:10.3847/0004-637X/820/2/99.

[375] Barber RJ, Strange JK, Hill C, Polyansky OL, Mellau GC, Yurchenko SN, et al. ExoMol line lists-III. An improved hot rotation-vibration line list for $\mathrm{HCN}$ and HNC. Mon Not R Astron Soc 2014;437:1828-35. doi:10.1093/mnras/stt2011.

[376] Tennyson J, Yurchenko SN. ExoMol: molecular line lists for exoplanet and other atmospheres. Mon Not R Astron Soc 2012;425:21-33. doi:10.1111/j. 1365-2966.2012.21440.x

[377] Mellau GC. Complete experimental rovibrational eigenenergies of HCN up to $6880 \mathrm{~cm}^{-1}$ above the ground state. J Chem Phys 2011;134:234303. doi:10. $1063 / 1.3598942$.

[378] Mellau GC. Rovibrational eigenenergy structure of the $[\mathrm{H}, \mathrm{C}, \mathrm{N}]$ molecular system. J Chem Phys 2011;134:194302. doi:10.1063/1.3590026.

[379] Harris GJ, Tennyson J, Kaminsky BM, Pavlenko YV, Jones HRA. Improved $\mathrm{HCN} / \mathrm{HNC}$ linelist, model atmospheres and synthetic spectra for WZ Cas. Mon Not R Astron Soc 2006;367:400-6. doi:10.1111/j.1365-2966.2005.09960.x.

[380] van Mourik T, Harris GJ, Polyansky OL, Tennyson J, Császár AG, Knowles PJ. $\mathrm{Ab}$ initio global potential, dipole, adiabatic, and relativistic correction surfaces for the HCN-HNC system. J Chem Phys 2001;115:3706-18. doi:10.1063/ 1.1383586 .

[381] Harris GJ, Polyansky OL, Tennyson J. Ab initio rotation-vibration spectra of HCN and HNC. Spectrochim Acta Part A Mol Biomol Spectrosc 2002;58:67390. doi:10.1016/S1386-1425(01)00664-3.

[382] Yang C, Buldyreva J, Gordon IE, Rohart F, Cuisset A, Mouret G, et al. Oxygen, nitrogen and air broadening of $\mathrm{HCN}$ spectral lines at terahertz frequencies. J Quant Spectrosc Radiat Transf 2008;109:2857-68. doi:10.1016/j.jqsrt.2008.08. 005 .

[383] Nikitin AV, Dmitrieva TA, Gordon IE. Improved spectroscopic line list of methyl chloride in the $1900-2600 \mathrm{~cm}^{-1}$ spectral region. J Quant Spectrosc Radiat Transf 2015. doi:10.1016/j.jqsrt.2016.03.007

[384] Dudaryonok AS, Lavrentieva NN, Buldyreva JV. $\mathrm{CH} 3 \mathrm{Cl}$ self-broadening coefficients and their temperature dependence. J Quant Spectrosc Radiat Transf 2013;130:321-6. doi:10.1016/j.jqsrt.2013.07.013.

[385] Bray C, Jacquemart D, Lacome N, Guinet M, Cuisset A, Eliet S, et al. Analysis of self-broadened pure rotational and rovibrational lines of methyl chloride at room temperature. J Quant Spectrosc Radiat Transf 2013;116:87-100. doi:10. 1016/j.jqsrt.2012.09.013

[386] Barbouchi Ramchani A, Jacquemart D, Dhib M, Aroui H. Line positions, intensities and self-broadening coefficients for the $v_{5}$ band of methyl chloride. J Quant Spectrosc Radiat Transf 2013;120:1-15. doi:10.1016/j.jqsrt.2013.02.002.

[387] Buldyreva J. Air-broadening coefficients of $\mathrm{CH}_{3}{ }^{35} \mathrm{Cl}$ and $\mathrm{CH}_{3}{ }^{37} \mathrm{Cl}$ rovibrational lines and their temperature dependence by a semi-classical approach. J Quant Spectrosc Radiat Transf 2013;130:315-20. doi:10.1016/j.jqsrt.2013.04.003.

[388] Guinet M, Rohart F, Buldyreva J, Gupta V, Eliet S, Motiyenko RA, et al. Experimental studies by complementary terahertz techniques and semi-classical calculations of $\mathrm{N}_{2}$ - broadening coefficients of $\mathrm{CH}_{3}{ }^{35} \mathrm{Cl}$. J Quant Spectrosc Radiat Transf 2012;113:1113-26. doi:10.1016/j.jqsrt.2012.01.022.

[389] Buldyreva J, Margulès L, Motiyenko RA, Rohart F. Speed dependence of $\mathrm{CH}_{3}{ }^{35} \mathrm{Cl}-\mathrm{O} 2$ line-broadening parameters probed on rotational transitions: measurements and semi-classical calculations. J Quant Spectrosc Radiat Transf 2013;130:304-14. doi:10.1016/j.jqsrt.2013.05.023.

[390] Sato TO, Mizoguchi A, Mendrok J, Kanamori H, Kasai Y. Measurement of the pressure broadening coefficient of the $625 \mathrm{GHz}$ transition of $\mathrm{H}_{2} \mathrm{O}_{2}$ in the sub-millimeter-wave region. J Quant Spectrosc Radiat Transf 2010;111:821-5. doi:10.1016/j.jqsrt.2009.11.022.

[391] Goyette TM, Ebenstein WL, Shostak SL, De Lucia FC, Helminger P. Pressure broadening of $\mathrm{NO}_{2}, \mathrm{CF}_{2} \mathrm{Cl}_{2}, \mathrm{HDO}$ and $\mathrm{HOOH}$ by $\mathrm{O}_{2}$ and $\mathrm{N}_{2}$ in the millimeter wave region. J Quant Spectrosc Radiat Transf 1988;40:129-34. doi:10.1016/ 0022-4073(88)90150-1.
[392] Devi VM, Rinsland CP, Smith Ma, Benner DC, Fridovich B. Tunable diode laser measurements of air-broadened linewidths in the $v_{6}$ band of $\mathrm{H}_{2} \mathrm{O}_{2}$. Appl Opt 1986;25:1844-7. doi:10.1364/A0.25.001844.

[393] Al-Refaie AF, Polyansky OL, Ovsyannikov RI, Tennyson J, Yurchenko SN. ExoMol line lists-XV. A new hot line list for hydrogen peroxide. Mon Not $\mathrm{R}$ Astron Soc 2016;461:1012-22. doi:10.1093/mnras/stw1295.

[394] Al-Refaie AF, Ovsyannikov RI, Polyansky OL, Yurchenko SN, Tennyson J. A variationally calculated room temperature line-list for $\mathrm{H}_{2} \mathrm{O}_{2}$. J Mol Spectrosc 2015;318:84-90. doi:10.1016/j.jms.2015.10.004.

[395] Lyulin OM, Perevalov VI. Global modeling of vibration-rotation spectra of the acetylene molecule. J Quant Spectrosc Radiat Transf 2016;177:59-74. doi:10. 1016/j.jqsit.2015.12.021.

[396] Drouin BJ, Yu S. Acetylene spectra near $2.6 \mathrm{THz}$. J Mol Spectrosc 2011;269:254-6. doi:10.1016/j.jms.2011.06.004.

[397] Jacquemart D, Lyulin OM, Perevalov VI. Recommended acetylene line list in the $20-240 \mathrm{~cm}^{-1}$ and $400-630 \mathrm{~cm}^{-1}$ regions: new measurements and global modeling. J Quant Spectrosc Radiat Transf 2017 this issue. doi:10.1016/ j.jqsrt.2017.03.008

[398] Jacquemart D, Mandin J-Y, Dana V, Régalia-Jarlot L, Plateaux J-J, Décatoire D, et al. The spectrum of acetylene in the 5- $\mu \mathrm{m}$ region from new line-parameter measurements. J Quant Spectrosc Radiat Transf 2003;76:237-67. doi:10.1016/ S0022-4073(02)00055-9.

[399] Lambot D, Blanquet G, Walrand J, Bouanich J-P. Diode-laser measurements of $\mathrm{H}_{2}$-broadening coefficients in the $\nu_{5}$ band of $\mathrm{C}_{2} \mathrm{H}_{2}$. J Mol Spectrosc 1991;150:164-72. doi:10.1016/0022-2852(91)90199-K.

[400] Thibault F, Corretja B, Viel A, Bermejo D, Martínez RZ, Bussery-Honvault B. Linewidths of $\mathrm{C}_{2} \mathrm{H}_{2}$ perturbed by $\mathrm{H}_{2}$ : experiments and calculations from an ab initio potential. Phys Chem Chem Phys 2008;10:5419. doi:10.1039/ b804306j.

[401] Thibault F, Ivanov SV, Buzykin OG, Gomez L, Dhyne M, Joubert P, et al. Comparison of classical, semiclassical and quantum methods in hydrogen broadening of acetylene lines. J Quant Spectrosc Radiat Transf 2011;112:1429-37. doi:10.1016/j.jqsrt.2011.02.011.

[402] Babay A, Ibrahimi M, Lemaire V, Lemoine B, Rohart F, Bouanich JP. Line frequency shifting in the $\nu_{5}$ band of $\mathrm{C}_{2} \mathrm{H}_{2}$. J Quant Spectrosc Radiat Transf 1998;59:195-202. doi:10.1016/S0022-4073(97)00122-2.

[403] Thibault F. Theoretical He-broadening coefficients of infrared and Raman $\mathrm{C}_{2} \mathrm{H}_{2}$ lines and their temperature dependence. J Mol Spectrosc 2005;234:286-8. doi:10.1016/j.jms.2005.09.008.

[404] Bond KS, Collett ND, Fuller EP, Hardwick JL, Hinds EE, Keiber TW, et al. Temperature dependence of pressure broadening and shifts of acetylene at $1550 \mathrm{~nm}$ by He, Ne, and Ar. Appl Phys B 2008;90:255-62. doi:10.1007| s00340-007-2842-0.

[405] Martin B, Walrand J, Blanquet G, Bouanich J-P, Lepère $\mathrm{M} . \mathrm{CO}_{2}$-broadening coefficients in the $v_{4}+v_{5}$ band of acetylene. J Mol Spectrosc 2006;236:52-7. doi:10.1016/j.jms.2005.12.011.

[406] Minutolo P, Corsi C, D'Amato F, De Rosa M. Self- and foreign-broadening and shift coefficients for $\mathrm{C}_{2} \mathrm{H}_{2}$ lines at $1.54 \mu \mathrm{m}$. Eur Phys J D 2001;17:175-9. doi:10.1007/s100530170020.

[407] Lyulin OM, Campargue A. An empirical spectroscopic database for acetylene in the regions of 5850-6341 $\mathrm{cm}^{-1}$ and $7000-9415 \mathrm{~cm}^{-1}$. J Quant Spectrosc Radiat Transf 2017. doi:10.1016/j.jqsrt.2017.01.036.

[408] Sada PV, Mccabe GH, Bjoraker GL, Jennings DE, Reuter DC. ${ }^{13} \mathrm{C}$-Ethane in the atmospheres of Jupiter and Saturn. Astrophys J 1996;472:903-7. doi:10.1086/ 178120.

[409] Flasar FM. Titan's atmospheric temperatures, winds, and composition. Science (80-) 2005;308:975-8. doi:10.1126/science.1111150.

[410] Jennings D.E. Private communication 2015.

[411] Malathy Devi V, Chris Benner D, Rinsland CP, Smith MAH, Sams RL, Blake TA et al. Multispectrum measurements of spectral line parameters including temperature dependences of $\mathrm{N}_{2}$ - and self-broadened half-width coefficients in the region of the $v_{9}$ band of ${ }^{12} \mathrm{C}_{2} \mathrm{H}_{6}$. J Quant Spectrosc Radiat Transf 2010;111:2481-504. doi:10.1016/j.jqsit.2010.07.010.

[412] Malathy Devi V, Rinsland CP, Chris Benner D, Sams RL, Blake TA. Multispectrum analysis of the $v_{9}$ band of ${ }^{12} \mathrm{C}_{2} \mathrm{H}_{6}$ : positions, intensities, selfand $\mathrm{N}_{2}$-broadened half-width coefficients. J Quant Spectrosc Radiat Transf 2010;111:1234-51. doi:10.1016/j.jqsrt.2009.10.017

[413] Di Lauro C, Lattanzi F, Brown LR, Sung K, Vander Auwera J, Mantz AW, et al. High resolution investigation of the $7 \mu \mathrm{m}$ region of the ethane spectrum. Planet Space Sci 2012;60:93-101. doi:10.1016/j.pss.2011.01.008.

[414] Moazzen-Ahmadi N, Norooz Oliaee J, Ozier I, Wishnow EH, Sung K, Crawford TJ, et al. An intensity study of the torsional bands of ethane at $35 \mu \mathrm{m}$. J Quant Spectrosc Radiat Transf 2015;151:123-32. doi:10.1016/j.jqsrt.2014.09. 016.

[415] Moazzen-Ahmadi N. A combined frequency analysis of the $v_{3}, v_{9}, 3 v_{4}$ and the far-infrared bands of ethane: a reassessment of the torsional parameters for the ground vibrational state. J Mol Spectrosc 2002;214:144-51. doi:10.1006/jmsp.2002.8599.

[416] Devi VM, Benner DC, Sung K, Crawford TJ, Mantz AW, Smith MAH. Line positions and intensities for the $v_{12}$ band of ${ }^{13} \mathrm{C}^{12} \mathrm{CH}_{6}$. J Mol Spectrosc 2014;301:28-38. doi:10.1016/j.jms.2014.05.005.

[417] Daly AM, Drouin BJ, Pearson JC, Sung K, Brown LR, Mantz A, et al. The $v_{17}$ band of $\mathrm{C}_{2} \mathrm{H}_{5} \mathrm{D}$ from 770 to $880 \mathrm{~cm}^{-1}$. J Mol Spectrosc 2015;316:1-10. doi:10. 1016/j.jms.2015.06.006. 
[418] di Lauro C, Lattanzi F, Brown LR, Sung K, Mantz AW, Smith MAH. The $v_{4}, v_{9}$, $v_{10}$ and $v_{6}+v_{11}$ bands of ${ }^{12} \mathrm{CH}_{3}{ }^{13} \mathrm{CH}_{3}$ between 1345 and $1557 \mathrm{~cm}^{-1}$. J Mol Spectrosc 2014;302:36-49. doi:10.1016/j.jms.2014.06.001.

[419] Kunde V, Hanel R, Maguire W, Gautier D, Baluteau JP, Marten A, et al. The tropospheric gas composition of Jupiter's north equatorial belt $/ \mathrm{NH}_{3}, \mathrm{PH}_{3}, \mathrm{CH}_{3} \mathrm{D}$, $\mathrm{GeH}_{4}, \mathrm{H}_{2} \mathrm{O} /$ and the Jovian $\mathrm{D} / \mathrm{H}$ isotopic ratio. Astrophys J 1982;263:443. doi:10.1086/160516.

[420] Noll K. The spectrum of Saturn from 1990 to $2230 \mathrm{~cm}^{-1}$ : abundances of $\mathrm{AsH}_{3}, \mathrm{CH}_{3} \mathrm{D}, \mathrm{CO}, \mathrm{GeH}_{4}, \mathrm{NH}_{3}$, and $\mathrm{PH}_{3}$. Icarus 1991;89:168-89. doi:10.1016/ 0019-1035(91)90096-C.

[421] Ridgway ST, Wallace L, Smith GR. The 800-1200 inverse centimeter absorption spectrum of Jupiter. Astrophys J 1976;207:1002. doi:10.1086/154570.

[422] Larson HP, Fink U, Smith HA, Davis DS. The middle-infrared spectrum of Saturn-evidence for phosphine and upper limits to other trace atmospheric constituents. Astrophys J 1980;240:327. doi:10.1086/158236.

[423] Sousa-Silva C, Yurchenko SN, Tennyson J. A computed room temperature line list for phosphine. J Mol Spectrosc 2013;288:28-37. doi:10.1016/j.jms.2013.04. 002.

[424] Sousa-Silva C, Al-Refaie AF, Tennyson J, Yurchenko SN. ExoMol line lists-VII. The rotation-vibration spectrum of phosphine up to $1500 \mathrm{~K}$. Mon Not R Astron Soc 2014:446:2337-47. doi:10.1093/mnras/stu2246.

[425] Malathy Devi V, Benner DC, Kleiner I, Sams RL, Fletcher LN. Line shape parameters of $\mathrm{PH}_{3}$ transitions in the Pentad near 4-5 $\mu \mathrm{m}$ : selfbroadened widths, shifts, line mixing and speed dependence. J Mol Spectrosc 2014;302:17-33. doi:10.1016/j.jms.2014.06.003.

[426] Malathy Devi V, Kleiner I, Sams RL, Brown LR, Benner DC, Fletcher LN. Line positions and intensities of the phosphine $\left(\mathrm{PH}_{3}\right)$ Pentad near $4.5 \mu \mathrm{m}$. J Mol Spectrosc 2014;298:11-23. doi:10.1016/j.jms.2014.01.013.

[427] Nikitin AV, Ivanova YA, Rey M, Tashkun SA, Toon GC, Sung K, et al. Analysis of $\mathrm{PH}_{3}$ spectra in the octad range $2733-3660 \mathrm{~cm}^{-1}$. J Quant Spectrosc Radiat Transf 2017:1-8 this issue. doi:10.1016/j.jqsrt.2017.04.032

[428] Nikitin AV, Holka F, Tyuterev VG, Fremont J. Vibration energy levels of the $\mathrm{PH}_{3}, \mathrm{PH}_{2} \mathrm{D}$, and $\mathrm{PHD}_{2}$ molecules calculated from high order potential energy surface. J Chem Phys 2009;130:244312. doi:10.1063/1.3156311.

[429] Nikitin AV, Rey M, Tyuterev VG. High order dipole moment surfaces of $\mathrm{PH}_{3}$ and $\mathrm{ab}$ initio intensity predictions in the Octad range. J Mol Spectrosc 2014;305:40-7. doi:10.1016/j.jms.2014.09.010.

[430] Rey M, Nikitin AV, Tyuterev VG. Ab initio ro-vibrational Hamiltonian in irreducible tensor formalism: a method for computing energy levels from potential energy surfaces for symmetric-top molecules. Mol Phys 2010;108:212135. doi:10.1080/00268976.2010.506892.

[431] Ulenikov ON, Malikova AB, Koivusaari M, Alanko S, Anttila R. High resolution vibrational-rotational spectrum of $\mathrm{H}_{2} \mathrm{~S}$ in the region of the $v_{2}$ fundamental band. J Mol Spectrosc 1996;176:229-35. doi:10.1006/jmsp.1996.0082.

[432] Flaud J-M, Camy-Peyret C, Johns JWC. The far-infrared spectrum of hydrogen sulfide. The (000) rotational constants of $\mathrm{H}_{2}{ }^{32} \mathrm{~S}, \mathrm{H}_{2}{ }^{33} \mathrm{~S}$, and $\mathrm{H}_{2}{ }^{34} \mathrm{~S}$. Can J Phys 1983;61:1462-73. doi:10.1139/p83-188.

[433] Brown LR, Crisp JA, Crisp D, Naumenko OV, Smirnov MA, Sinitsa LN, et al. The absorption spectrum of $\mathrm{H}_{2} \mathrm{~S}$ between 2150 and $4260 \mathrm{~cm}^{-1}$ : analysis of the positions and intensities in the first $\left(2 v_{2}, v_{1}\right.$, and $\left.v_{3}\right)$ and second $\left(3 v_{2}\right.$, $v_{1}+v_{2}$, and $\left.v_{2}+v_{3}\right)$. J Mol Spectrosc 1998;188:148-74. doi:10.1006/jmsp.1997. 7501.

[434] Rothman LS, Barbe A, Chris Benner D, Brown LR, Camy-Peyret C, Carleer MR, et al. The HITRAN molecular spectroscopic database: edition of 2000 including updates through 2001. J Quant Spectrosc Radiat Transf 2003;82:5-44. doi:10.1016/S0022-4073(03)00146-8.

[435] Waschull J, Kuhnemann F, Sumpf B. Self-, air, and helium broadening in the $v_{2}$ band of $\mathrm{H}_{2} \mathrm{~S}$. J Mol Spectrosc 1994;165:150-8. doi:10.1006/jmsp. 1994.1117

[436] Sumpf B, Meusel I, Kronfeldt H-D. Self- and air-broadening in the $v_{1}$ and $v_{3}$ bands of $\mathrm{H}_{2} \mathrm{~S}$. J Mol Spectrosc 1996;177:143-5. doi:10.1006/jmsp.1996.0126.

[437] Kissel A, Sumpf B, Kronfeldt H-D, Tikhomirov B, Ponomarev Y. Molecular-GasPressure-induced line-shift and line-broadening in the $v_{2}$-band of $\mathrm{H}_{2} \mathrm{~S}$. J Mol Spectrosc 2002;216:345-54. doi:10.1006/jmsp.2002.8630.

[438] Sumpf B. Experimental investigation of the self-broadening coefficients in the $v_{1}+v_{3}$ band of $\mathrm{SO}_{2}$ and the $2 v_{2}$ band of $\mathrm{H}_{2} \mathrm{~S}$. J Mol Spectrosc 1997;181:1607. doi:10.1006/jmsp.1996.7168.

[439] Tan Y, Kochanov RV, Rothman LS, Gordon IE. The broadening coefficients of $\mathrm{H}_{2} \mathrm{~S}$ 2016. doi: $10.5281 /$ zenodo.345381

[440] Ball CD, Dutta JM, Beaky MM, Goyette TM, De Lucia FC. Variable-temperature pressure broadening of $\mathrm{H}_{2} \mathrm{~S}$ by $\mathrm{O}_{2}$ and $\mathrm{N}_{2}$. J Quant Spectrosc Radiat Transf 1999;61:775-80. doi:10.1016/S0022-4073(98)00065-X.

[441] Ciaffoni L, Cummings BL, Denzer W, Peverall R, Procter SR, Ritchie GAD. Line strength and collisional broadening studies of hydrogen sulphide in the $1.58 \mu \mathrm{m}$ region using diode laser spectroscopy. Appl Phys B 2008;92:627-33. doi:10.1007/s00340-008-3119-y.

[442] Nelson DD, Zahniser MS. Air-broadened linewidth measurements in the $v_{2}$ vibrational band of the hydroperoxyl radical. J Mol Spectrosc 1994;166:2739. doi:10.1006/jmsp.1994.1193.

[443] Minamida M, Tonokura K. Air broadening coefficients for the $v_{3}$ band of hydroperoxyl radicals. J Quant Spectrosc Radiat Transf 2014;148:65-9. doi:10. 1016/j.jqsrt.2014.06.020.

[444] Mizoguchi A, Yagi T, Kondo K, Sato TO, Kanamori H. Submillimeter-wave measurements of $\mathrm{N}_{2}$ and $\mathrm{O}_{2}$ pressure broadening for $\mathrm{HO}_{2}$ radical generated by Hg-photosensitized reaction. J Quant Spectrosc Radiat Transf 2012;113:27985. doi:10.1016/j.jqsrt.2011.11.009.
[445] Miyano S, Tonokura K. Measurements of nitrogen-broadening coefficients in the $v_{3}$ band of the hydroperoxyl radical using a continuous wave quantum cascade laser. J Mol Spectrosc 2011;265:47-51. doi:10.1016/j.jms. 2010.10.010.

[446] Chance K, Denatale P, Bellini M, Inguscio M, Dilonardo G, Fusina L. Pressure broadening of the $2.4978-\mathrm{THz}$ rotational lines of $\mathrm{HO}_{2}$ by $\mathrm{N}_{2}$ and $\mathrm{O}_{2}$. J Mol Spectrosc 1994;163:67-70. doi:10.1006/jmsp.1994.1007.

[447] Ibrahim N, Thiebaud J, Orphal J, Fittschen C. Air-broadening coefficients of the $\mathrm{HO}_{2}$ radical in the $2 v_{1}$ band measured using cw-CRDS. J Mol Spectrosc 2007;242:64-9. doi:10.1016/j.jms.2007.02.007.

[448] Tan Y, Kochanov RV, Rothman LS, Gordon IE. The air-broadening coefficients of $\mathrm{HO}_{2}$. 2016. doi:10.5281/zenodo.35917.

449] Rothman LS, Rinsland CP, Goldman A, Massie ST, Edwards DP, Flaud J-M, et al. The HITRAN molecular spectroscopic database and HAWKS (HITRAN Atmospheric Workstation): 1996 edition. J Quant Spectrosc Radiat Transf 1998;60:665-710. doi:10.1016/S0022-4073(98)00078-8.

[450] Zink LR, Evenson KM, Matsushima F, Nelis T, Robinson RL. Atomic oxygen fine-structure splittings with tunable far-infrared spectroscopy. Astrophys J 1991;371:L85. doi:10.1086/186008.

[451] Pickett HM, Poynter RL, Cohen EA, Delitsky ML, Pearson JC, Müller HSP. Submillimeter, millimeter, and microwave spectral line catalog. J Quant Spectrosc Radiat Transf 1998;60:883-90. doi:10.1016/S0022-4073(98)00091-0.

[452] López-Puertas M, Flaud J-M, Peralta-Calvillo J, Funke B, Gil-López S. NO+ fundamental and first hot ro-vibrational line frequencies from MIPAS/Envisat atmospheric spectra. J Mol Spectrosc 2006;237:218-24. doi:10.1016/j.jms.2006. 03.015

[453] Cernicharo J, Bailleux S, Alekseev E, Fuente A, Roueff E, Gerin M, et al. Tentative detection of the nitrosylium ion in space. Astrophys J 2014;795:40. doi:10.1088/0004-637X/795/1/40.

[454] Endres CP, Schlemmer S, Schilke P, Stutzki J, Müller HSP. The Cologne Database for Molecular Spectroscopy, CDMS, in the Virtual Atomic and Molecular Data Centre, VAMDC. J Mol Spectrosc 2016;327:95-104. doi:10. 1016/j.jms.2016.03.005

[455] Bowman WC, Herbst E, De Lucia FC. Millimeter and submillimeter spectrum of $\mathrm{NO}^{+}$. J Chem Phys 1982;77:4261-2. doi:10.1063/1.444307.

[456] Ho WC, Ozier I, Cramb DT, Gerry MCL. Diode laser spectroscopy of the vibrational fundamental of $\mathrm{NO}^{+}$. J Mol Spectrosc 1991;149:559-61. doi:10.1016 0022-2852(91)90311-W.

[457] Polák R, Fišer J. A comparative icMRCI study of some $\mathrm{NO}^{+}$, NO and $\mathrm{NO}^{-}$ electronic ground state properties. Chem Phys 2004;303:73-83. doi:10.1016/ j.chemphys.2004.04.027.

[458] Sawada S, Totsuka T. Natural and anthropogenic sources and fate of atmospheric ethylene. Atmos Environ 1986;20:821-32. doi:10.1016/0004-6981(86) 90266-0.

459] Hesman BE, Bjoraker GL, Sada PV, Achterberg RK, Jennings DE, Romani PN, et al. Elusive ethylene detected in Saturn's northern storm region. Astrophys J 2012;760:24. doi:10.1088/0004-637X/760/1/24.

[460] Vander Auwera J, Fayt A, Tudorie M, Rotger M, Boudon V, Franco B, et al. Self-broadening coefficients and improved line intensities for the $\nu_{7}$ band of ethylene near $10.5 \mu \mathrm{m}$, and impact on ethylene retrievals from Jungfraujoch solar spectra. J Quant Spectrosc Radiat Transf 2014;148:177-85. doi:10.1016/j. jqsit.2014.07.003.

[461] Alkadrou A, Bourgeois M-T, Rotger M, Boudon V, Vander Auwera J. Global frequency and intensity analysis of the $v_{10} / v_{7} / v_{4} / v_{12}$ band system of ${ }^{12} \mathrm{C}_{2} \mathrm{H}_{4}$ at $10 \mu \mathrm{m}$ using the $\mathrm{D}_{2 \mathrm{~h}}$ Top Data System. J Quant Spectrosc Radiat Transf 2016;182:158-71. doi:10.1016/j.jqsrt.2016.05.024.

[462] Willaert F, Demaison J, Margules L, Mäder H, Spahn H, Giesen T, et al. The spectrum of ethylene from microwave to submillimetre-wave. Mol Phys 2006;104:273-92. doi:10.1080/00268970500273314.

[463] Cauuet I, Walrand J, Blanquet G, Valentin a, Henry L, Lambeau C, et al. Extension to third-order Coriolis terms of the analysis of $v_{10}, v_{7}$, and $v_{4}$ levels of ethylene on the basis of Fourier transform and diode laser spectra. J Mol Spectrosc 1990;139:191-214. doi:10.1016/0022-2852(90)90251-K.

[464] Legrand J, Azizi M, Herlemont F, Fayt A. Saturation spectroscopy of $\mathrm{C}_{2} \mathrm{H}_{4}$ using a $\mathrm{CO}_{2}$ laser sideband spectrometer. J Mol Spectrosc 1995;171:13-21. doi:10.1006/jmsp.1995.1099.

[465] Rusinek E, Fichoux H, Khelkhal M, Herlemont F, Legrand J, Fayt A. Subdoppler study of the $v_{7}$ band of $\mathrm{C}_{2} \mathrm{H}_{4}$ with a $\mathrm{CO}_{2}$ laser sideband spectrometer. $\mathrm{J} \mathrm{Mol}$ Spectrosc 1998;189:64-73. doi:10.1006/jmsp.1998.7539.

[466] Blass WE, Jennings L, Ewing AC, Daunt SJ, Weber MC, Senesac L, et al. Absolute intensities in the $v_{7}$ band of ethylene: tunable laser measurements used to calibrate FTS broadband spectra. J Quant Spectrosc Radiat Transf 2001;68:467-72. doi:10.1016/S0022-4073(00)00050-9.

[467] Rotger M, Boudon V, Vander Auwera J. Line positions and intensities in the $v_{12}$ band of ethylene near $1450 \mathrm{~cm}^{-1}$ : an experimental and theoretical study. J Quant Spectrosc Radiat Transf 2008;109:952-62. doi:10.1016/j.jqsrt.2007.12. 005 .

[468] Alkadrou A, Bourgeois M-T, Rotger M, Boudon V, Vander Auwera J. Corrigendum to "Global frequency and intensity analysis of the $v_{10} / v_{7} / v_{4} / v_{12}$ band system of ${ }^{12} \mathrm{C}_{2} \mathrm{H}_{4}$ at $10 \mu \mathrm{m}$ using the D2h top data system. J Quant Spectrosc Radiat Transf 2017;190:88. doi:10.1016/j.jqsrt.2017.01.007.

[469] Rey M, Delahaye T, Nikitin A V, Tyuterev VG. First theoretical global line lists of ethylene $\left({ }^{12} \mathrm{C}_{2} \mathrm{H}_{4}\right)$ spectra for the temperature range $50-700 \mathrm{~K}$ in the far-infrared for quantification of absorption and emission in planetary atmospheres. Astron Astrophys 2016;594:A47. doi:10.1051/0004-6361/ 201629004 
[470] Delahaye T, Nikitin A, Rey M, Szalay PG, Tyuterev VG. A new accurate groundstate potential energy surface of ethylene and predictions for rotational and vibrational energy levels. J Chem Phys 2014;141. doi:10.1063/1.4894419.

[471] Delahaye T, Nikitin AV, Rey M, Szalay PG, Tyuterev VG. Accurate 12D dipole moment surfaces of ethylene. Chem Phys Lett 2015;639:275-82. doi:10.1016/ j.cplett.2015.09.042.

[472] Flaud J-M, Lafferty WJ, Malathy Devi V, Sams RL, Chris Benner D. Absolute line intensities and self-broadened half-width coefficients in the ethylene$1-^{13} \mathrm{C}$ bands in the $700-1190 \mathrm{~cm}^{-1}$ region. J Mol Spectrosc 2011;267:3-12. doi:10.1016/j.jms.2011.01.002.

[473] Flaud J-M, Lafferty WJ, Sams R, Malathy Devi V. High resolution analysis of the ethylene-1-13 $\mathrm{C}$ spectrum in the $8.4-14.3-\mu \mathrm{m}$ region. J Mol Spectrosc 2010;259:39-45. doi:10.1016/j.jms.2009.10.003.

[474] Kleinböhl A, Toon GC, Sen B, Blavier JFL, Weisenstein DK, Wennberg PO. Infrared measurements of atmospheric $\mathrm{CH}_{3} \mathrm{CN}$. Geophys Res Lett 2005;32:1-5. doi:10.1029/2005GL024283.

[475] Remijan AJ, Milam SN, Womack M, Apponi AJ, Ziurys LM, Wyckoff S, et al. The distribution, excitation, and formation of cometary molecules: methanol, methyl cyanide, and ethylene glycol. Astrophys J 2008;689:613-21. doi:10. $1086 / 592242$.

[476] Marten A. New millimeter heterodyne observations of Titan: vertical distributions of nitriles $\mathrm{HCN}, \mathrm{HC}_{3} \mathrm{~N}, \mathrm{CH}_{3} \mathrm{CN}$, and the isotopic ratio ${ }^{15} \mathrm{~N} /{ }^{14} \mathrm{~N}$ in its atmosphere. Icarus 2002;158:532-44. doi:10.1006/icar.2002.6897.

[477] Rinsland CP, Malathy Devi V, Chris Benner D, Blake TA, Sams RL, Brown LR, et al. Multispectrum analysis of the $v_{4}$ band of $\mathrm{CH}_{3} \mathrm{CN}$ : Positions, intensities, self- and $\mathrm{N}_{2}$-broadening, and pressure-induced shifts. J Quant Spectrosc Radiat Transf 2008;109:974-94. doi:10.1016/j.jqsrt.2007.11.013.

[478] Müller HSP. https://molspect.chemistry.ohio-state.edu/symposium_65/ symposium/Abstracts/p003.pdf.

[479] Müller HSP, Brown LR, Drouin BJ, Pearson JC, Kleiner I, Sams RL, et al. Rotational spectroscopy as a tool to investigate interactions between vibrational polyads in symmetric top molecules: Low-lying states $\mathrm{v}_{8}<3$ of methyl cyanide $\mathrm{CH}_{3} \mathrm{CN}$. J Mol Spectrosc 2015;312:22-37. doi:10.1016/j.jms.2015.02. 009.

[480] Koivusaari M, Horneman V-M, Anttila R. High-resolution study of the infrared band $v_{8}$ of $\mathrm{CH}_{3}$ CN. J Mol Spectrosc 1992;152:377-88. doi:10.1016/ 0022-2852(92)90076-Z.

[481] Cazzoli G, Puzzarini C. The Lamb-dip spectrum of methylcyanide: precise rotational transition frequencies and improved ground-state rotational parameters. J Mol Spectrosc 2006;240:153-63. doi:10.1016/j.jms.2006.09.013.

[482] Müller HSP, Drouin BJ, Pearson JC. Rotational spectra of isotopic species of methyl cyanide, $\mathrm{CH}_{3} \mathrm{CN}$, in their ground vibrational states up to terahertz frequencies. Astron Astrophys 2009;506:1487-99. doi:10.1051/0004-6361/ 200912932.

[483] Anttila R, Horneman VM, Koivusaari M, Paso R. Ground State Constants $A_{0}$, $\mathrm{D}_{\mathrm{K} 0}$ and $\mathrm{H}_{\mathrm{K} 0}$ of $\mathrm{CH}_{3} \mathrm{CN}$. J Mol Spectrosc 1993;157:198-207. doi:10.1006/jmsp. 1993.1016.

[484] Bauer A, Tarrago G, Remy A. Analysis of the rotational spectrum of $C_{3 \mathrm{v}}$ molecules by using factorization and diagonalization of the energy matrix. J Mol Spectrosc 1975;58:111-24. doi:10.1016/0022-2852(75)90160-5.

[485] Tolonen AM, Koivusaari M, Paso R, Schroderus J, Alanko S, Anttila R. The infrared spectrum of methyl cyanide between 850 and $1150 \mathrm{~cm}^{-1}$ : analysis of the $v_{4}, v_{7}$, and $3 v_{18}$ bands with resonances. J Mol Spectrosc 1993;160:55465. doi:10.1006/jmsp.1993.1201

[486] Carlos M., Gruson O., Rotger M., Thomas X., Loëte M., Boudon V. Highresolution spectroscopy and analysis of various bands of $\mathrm{CF}_{4}$ to elucidate its hot band structure. 2017.

[487] Georges R. Private communication. 2016

[488] Jolly A, Fayt A, Benilan Y, Jacquemart D, Nixon CA. The $v_{8}$ bending mode of diacetylene: from laboratory spectroscopy to the detection of ${ }^{13} \mathrm{C}$ isotopologues in Titan's atmosphere. Astrophys J 2010;714:852-9. doi:10.1088/ 0004-637X/714/1/852.

[489] Jolly A, Manceron L, Kwabia-Tchana F, Benilan Y, Gazeau M-C. Revised infrared bending mode intensities for diacetylene $\left(\mathrm{C}_{4} \mathrm{H}_{2}\right)$ : Application to Titan. Planet Space Sci 2014;97:60-4. doi:10.1016/j.pss.2014.03.020

[490] Malek SE, Cami J, Bernard-Salas J. The rich circumstellar chemistry of Smp Lmc 11. Astrophys J 2012;744:16. doi:10.1088/0004-637X/744/1/16

[491] Pachucki K, Komasa J. Electric dipole rovibrational transitions in the HD molecule. Phys Rev A 2008;78:52503. doi:10.1103/PhysRevA.78.052503.

[492] Wolniewicz L, Simbotin I, Dalgarno A. Quadrupole transition probabilities for the excited rovibrational states of $\mathrm{H}_{2}$. Astrophys J Suppl Ser 1998;293. doi:10. $1086 / 313091$

[493] Thibault F, Wcisło P, Ciuryło R. A test of $\mathrm{H}_{2}$-He potential energy surfaces. Eur Phys J D 2016;70:236. doi:10.1140/epjd/e2016-70114-9.

[494] Michaud J-P, Krupitsky D, Grove JS, Anderson BS. Volcano related atmospheric toxicants in Hilo and Hawaii volcanoes national park: implications for human health. Neurotoxicology 2005;26:555-63. doi:10.1016/j.neuro.2004.12.004.

[495] Hieta T, Merimaa M. Simultaneous detection of $\mathrm{SO}_{2}, \mathrm{SO}_{3}$ and $\mathrm{H}_{2} \mathrm{O}$ using QCL spectrometer for combustion applications. Appl Phys B 2014;117:847-54. doi:10.1007/s00340-014-5896-9.

[496] Zhang X, Liang M-C, Montmessin F, Bertaux J-L, Parkinson C, Yung YL. Photolysis of sulphuric acid as the source of sulphur oxides in the mesosphere of Venus. Nat Geosci 2010;3:834-7. doi:10.1038/ngeo989.

[497] Zhang X, Liang MC, Mills FP, Belyaev DA, Yung YL. Sulfur chemistry in the middle atmosphere of Venus. Icarus 2012;217:714-39. doi:10.1016/j.icarus. 2011.06.016.
[498] Whitehill AR, Xie C, Hu X, Xie D, Guo H, Ono S. Vibronic origin of sulfur mass-independent isotope effect in photoexcitation of $\mathrm{SO}_{2}$ and the implications to the early earth's atmosphere. Proc Natl Acad Sci 2013;110:17697-702. doi:10.1073/pnas.1306979110.

[499] Ortigoso J, Escribano R, Maki AG. The $v_{2}$ and $v_{4}$ IR bands of $\mathrm{SO}_{3}$. J Mol Spectrosc 1989;138:602-13. doi:10.1016/0022-2852(89)90021-0.

[500] Kaldor A, Maki AG, Dorney AJ, Mills IM. The assignment of $v_{2}$ and $v_{4}$ of $\mathrm{SO}_{3}$ J Mol Spectrosc 1973;45:247-52. doi:10.1016/0022-2852(73)90155-0.

[501] Maki A, Blake Ta, Sams RL, Frieh J, Barber J, Masiello T, et al. Analysis of some combination-overtone infrared bands of ${ }^{32} \mathrm{~S}^{16} \mathrm{O}_{3}$. J Mol Spectrosc 2004;225:109-22. doi:10.1016/j.jms.2004.02.008.

[502] Maki A, Blake TA, Sams RL, Vulpanovici N, Barber J, Chrysostom ETH, et al. High-Resolution Infrared Spectra of the $v_{2}, v_{3}, v_{4}$, and $2 v_{3}$ Bands of ${ }^{32} \mathrm{~S}^{16} \mathrm{O}_{3}$. J Mol Spectrosc 2001;210:240-9. doi:10.1006/jmsp.2001.8454.

[503] Underwood DS, Tennyson J, Yurchenko SN. An ab initio variationally computed room-temperature line list for ${ }^{32} \mathrm{~S}^{16} \mathrm{O}_{3}$. Phys Chem Chem Phys 2013;15:10118, doi:10.1039/c3cp50303h.

[504] Meyer V, Sutter DH, Dreizler H. The centrifugally induced pure rotational spectrum and the structure of sulfur trioxide. A microwave Fourier transform study of a nonpolar molecule. Zeitschrift Für Naturforsch A 1991;46. doi:10.1515/zna-1991-0811.

[505] Underwood DS, Yurchenko SN, Tennyson J, Jensen P. Rotational spectrum of $\mathrm{SO}_{3}$ and theoretical evidence for the formation of sixfold rotational energylevel clusters in its vibrational ground state. J Chem Phys 2014;140. doi:10. 1063/1.4882865.

[506] Underwood DS, Yurchenko SN, Tennyson J, Al-Refaie AF, Clausen S, Fateev A. ExoMol molecular line lists-XVII. The rotation-vibration spectrum of hot $\mathrm{SO}_{3}$. Mon Not R Astron Soc 2016;462:4300-13. doi:10.1093/mnras/ stw1828.

[507] Teanby NA, Irwin PGJ, de Kok R, Jolly A, Bezard B, Nixon CA, et al. Titan's stratospheric $\mathrm{C}_{2} \mathrm{~N}_{2}, \mathrm{C}_{3} \mathrm{H}_{4}$, and $\mathrm{C}_{4} \mathrm{H}_{2}$ abundances from Cassini/CIRS far-infrared spectra. Icarus 2009;202:620-31. doi:10.1016/j.icarus.2009.03.022.

[508] Fayt A, Jolly A, Benilan Y, Manceron L, Kwabia-Tchana F, Guillemin J-C. Frequency and intensity analyses of the far infrared $v_{5}$ band system of cyanogen $\left(\mathrm{C}_{2} \mathrm{~N}_{2}\right)$ and applications to Titan. J Quant Spectrosc Radiat Transf 2012;113:1195-219. doi:10.1016/j.jqsrt.2012.02.003.

[509] Kiester E. An incomplete history of World War I. Pier 9, Murdoch Books: 2007.

[510] Fu D, Boone CD, Bernath PF, Walker KA, Nassar R, Manney GL, et al. Global phosgene observations from the atmospheric chemistry experiment (ACE) mission. Geophys Res Lett 2007;34:L17815. doi:10.1029/2007GL029942.

[511] Toon GC, Blavier J-F, Sen B, Drouin BJ. Atmospheric $\mathrm{COCl}_{2}$ measured by solar occultation spectrometry. Geophys Res Lett 2001;28:2835-8. doi:10.1029/ 2000GL012156.

[512] Valeri M, Carlotti M, Flaud J-M, Raspollini P, Ridolfi M, Dinelli BM. Phosgene in the UTLS: seasonal and latitudinal variations from MIPAS observations. Atmos Meas Tech Discuss 2016:1-19. doi:10.5194/amt-2016-62.

[513] Tchana FK, Lafferty WJ, Flaud J-M, Manceron L, Ndao M. High-resolution analysis of the $\nu_{1}$ and $\nu_{5}$ bands of phosgene ${ }^{35} \mathrm{Cl}_{2} \mathrm{CO}$ and ${ }^{35} \mathrm{Cl}^{37} \mathrm{ClCO}$. Mol Phys 2015;113:3241-6. doi:10.1080/00268976.2015.1015638.

[514] Clough SA, Shephard MW, Mlawer EJ, Delamere JS, Iacono MJ, Cady-Pereira K, et al. Atmospheric radiative transfer modeling: a summary of the AER codes. J Quant Spectrosc Radiat Transf 2005;91:233-44. doi:10.1016/j.jqsrt.2004.05. 058 .

[515] Chetwynd JH, Wang J, Anderson GP, Fast Atmospheric Signature CODE. (FASCODE): an update and applications in atmospheric remote sensing. In: Wang J, Hays PB, editors. SPIE's 1994 Int. Symp. Opt. Imaging, Instrum., International Society for Optics and Photonics; 1994. p. 613. doi:10.1117/12. 187599.

[516] Anderson GP, Kneizys FX, Chetwynd JH Jr, Rothman LS, Hoke ML, Berk A, et al. Reviewing atmospheric radiative transfer modeling: new developments in high- and moderate-resolution FASCODE/FASE and MODTRAN SPIE's 1996 Int Symp Opt Sci Eng Instrum, 82. editors. International Society for Optics and Photonics; 1996. doi:101117/12256105.

[517] Killinger DK, Wilcox WE, Pliutau D. HITRAN-PC: 25 years of academic development and commercialization of laser atmospheric transmission software for environmental remote sensing. Technol Innov 2012;14:303-27. doi:10. 3727/194982412X13500042169054

[518] Edwards P. GENLN2: A General Line-by-line Atmospheric Transmittance and Radiance Model. Version 3.0 Description and Users Guide 1992. doi:10.5065 D6W37T86.

[519] Johnson TJ, Profeta LTM, Sams RL, Griffith DWT, Yokelson RL. An infrared spectral database for detection of gases emitted by biomass burning. Vib Spectrosc 2010;53:97-102. doi:10.1016/j.vibspec.2010.02.010.

[520] Hodnebrog Ø, Etminan M, Fuglestvedt JS, Marston G, Myhre G, Nielsen CJ, et al. Global warming potentials and radiative efficiencies of halocarbons and related compounds: A comprehensive review. Rev Geophys 2013;51:300-78. doi:10.1002/rog.20013.

[521] Highwood E, Shine K. Radiative forcing and global warming potentials of 11 halogenated compounds. J Quant Spectrosc Radiat Transf 2000;66:169-83. doi:10.1016/S0022-4073(99)00215-0.

[522] Wagner G, Birk M. New infrared spectroscopic database for bromine nitrate. J Mol Spectrosc 2016;326:95-105. doi:10.1016/j.jms.2016.03.007.

[523] Harrison JJ. New and improved infrared absorption cross sections for dichlorodifluoromethane (CFC-12). Atmos Meas Tech 2015;8:3197-207. doi:10.5194/amt-8-3197-2015. 
[524] Harrison JJ. New and improved infrared absorption cross sections for chlorodifluoromethane (HCFC-22). Atmos Meas Tech 2016;9:2593-601. doi:10.5194/ amt-9-2593-2016.

[525] Harrison JJ, Boone CD, Bernath PF. New and improved infra-red absorption cross sections and ACE-FTS retrievals of carbon tetrachloride (CCl4). J Quant Spectrosc Radiat Transf 2017;186:139-49. doi:10.1016/j.jqsrt.2016.04.025.

[526] Harrison JJ. Infrared absorption cross sections for trifluoromethane. J Quant Spectrosc Radiat Transf 2013;130:359-64. doi:10.1016/j.jqsrt.2013.05.026.

[527] Harrison JJ. Infrared absorption cross sections for 1,1,1,2-tetrafluoroethane. J Quant Spectrosc Radiat Transf 2015;151:210-16. doi:10.1016/j.jqsrt.2014.09. 023.

[528] Reed ZD, Hodges JT. Self- and air-broadened cross sections of ethane $\left(\mathrm{C}_{2} \mathrm{H}_{6}\right)$ determined by frequency-stabilized cavity ring-down spectroscopy near $1.68 \mu \mathrm{m}$. J Quant Spectrosc Radiat Transf 2015;159:87-93. doi:10.1016/ j.jqsit.2015.03.010.

[529] Davis ME, Bernard F, McGillen MR, Fleming EL, Burkholder JB. UV and infrared absorption spectra, atmospheric lifetimes, and ozone depletion and global warming potentials for $\mathrm{CCl}_{2} \mathrm{FCCl}_{2} \mathrm{~F}$ (CFC-112), $\mathrm{CCl}_{3} \mathrm{CClF}_{2}$ (CFC112a), $\mathrm{CCl}_{3} \mathrm{CF}_{3}$ (CFC-113a), and $\mathrm{CCl}_{2} \mathrm{FCF}_{3}$ (CFC-114a). Atmos Chem Phys 2016;16:8043-52. doi:10.5194/acp-16-8043-2016.

[530] Etminan M, Highwood EJ, Laube JC, McPheat R, Marston G, Shine KP, et al. Infrared absorption spectra, radiative efficiencies, and global warming potentials of newly-detected halogenated compounds: CFC-113a, CFC-112 and HCFC-133a. Atmosphere (Basel) 2014:5:473-83. doi:10.3390/atmos5030473.

[531] Godin PJ, Cabaj A, Conway S, Hong AC, Le Bris K, Mabury SA, et al. Temperature-dependent absorption cross-sections of perfluorotributylamine. J Mol Spectrosc 2016;323:53-8. doi:10.1016/j.jms.2015.11.004.

[532] Godin PJ, Cabaj A, Xu L-H, Le Bris K, Strong K. A study of the temperature dependence of the infrared absorption cross-sections of 2,2,3,3,3pentafluoropropanol in the range of 298-362 K. J Quant Spectrosc Radiat Transf 2017:186:150-7, doi:10.1016/j.jqsrt.2016.05.031.

[533] Sellevag SR, D'Anna B, Nielsen CJ. Infrared absorption cross-sections and estimated global warming potentials of $\mathrm{CF}_{3} \mathrm{CH}_{2} \mathrm{CH}_{2} \mathrm{OH}, \mathrm{CHF}_{2} \mathrm{CF}_{2} \mathrm{CH}_{2} \mathrm{OH}$, $\mathrm{CF}_{3} \mathrm{CF}_{2} \mathrm{CH}_{2} \mathrm{OH}, \mathrm{CF}_{3} \mathrm{CHFCF}_{2} \mathrm{CH}_{2} \mathrm{OH}$, and $\mathrm{CF}_{3} \mathrm{CF}_{2} \mathrm{CF}_{2} \mathrm{CH}_{2} \mathrm{OH}$. Asian Chem Lett 2007:11:33-40.

[534] González S, Jiménez E, Ballesteros B, Martínez E, Albaladejo J. Hydroxyl radical reaction rate coefficients as a function of temperature and IR absorption cross sections for $\mathrm{CF}_{3} \mathrm{CH}=\mathrm{CH}_{2}$ (HFO-1243zf), potential replacement of $\mathrm{CF}_{3} \mathrm{CH}_{2} \mathrm{~F}$ (HFC-134a). Environ Sci Pollut Res 2014:4793-805. doi:10.1007/ s11356-014-3426-2.

[535] Le Bris K, Graham L. Quantitative comparisons of absorption cross-section spectra and integrated intensities of HFC-143a. J Quant Spectrosc Radiat Transf 2015;151:13-17. doi:10.1016/j.jqsrt.2014.09.005.

[536] McGillen MR, Bernard F, Fleming EL, Burkholder JB. HCFC-133a $\left(\mathrm{CF}_{3} \mathrm{CH}_{2} \mathrm{Cl}\right)$ : $\mathrm{OH}$ rate coefficient, UV and infrared absorption spectra, and atmospheric implications. Geophys Res Lett 2015;42:6098-105. doi:10.1002/2015GL064939.

[537] Papadimitriou VC, Burkholder JB. OH radical reaction rate coefficients, infrared spectrum, and global warming potential of $\left(\mathrm{CF}_{3}\right)_{2} \mathrm{CFCH}=\mathrm{CHF}$ (HFO1438ezy $(E))$. J Phys Chem A 2016;120:6618-28. doi:10.1021/acs.jpca.6b06096.

[538] Rodríguez A, Bravo I, Rodríguez D, Tajuelo M, Diaz-de-Mera Y, Aranda A. The environmental impact of unsaturated fluoroesters: atmospheric chemistry towards $\mathrm{OH}$ radicals and $\mathrm{Cl}$ atoms, radiative behavior and cumulative ozone creation. RSC Adv 2016;6:21833-43. doi:10.1039/C6RA00630B.

[539] Rodríguez A, Rodríguez D, Moraleda A, Bravo I, Moreno E, Notario A. Atmospheric chemistry of HFE-7300 and HFE-7500: temperature dependent kinetics, atmospheric lifetimes, infrared spectra and global warming potentials. Atmos Environ 2014;96:145-53. doi:10.1016/j.atmosenv.2014.07.033.

[540] Wallington TJ, Pivesso BP, Lira AM, Anderson JE, Nielsen CJ, Andersen NH, et al. $\mathrm{CH}_{3} \mathrm{Cl}, \mathrm{CH}_{2} \mathrm{Cl}_{2}, \mathrm{CHCl}_{3}$, and $\mathrm{CCl}_{4}$ : infrared spectra, radiative efficiencies, and global warming potentials. J Quant Spectrosc Radiat Transf 2016;174:5664. doi:10.1016/j.jqsrt.2016.01.029.

[541] Tereszchuk KA, Bernath PF. Infrared absorption cross-sections for acetaldehyde $\left(\mathrm{CH}_{3} \mathrm{CHO}\right)$ in the $3 \mu \mathrm{m}$ region. J Quant Spectrosc Radiat Transf 2011;112:990-3. doi:10.1016/j.jqsrt.2010.12.003.

[542] Sihra K, Hurley MD, Shine KP, Wallington TJ. Updated radiative forcing estimates of 65 halocarbons and nonmethane hydrocarbons. J Geophys Res 2001;106:20493-505. doi:10.1029/2000JD900716.

[543] Bravo I, Aranda A, Hurley MD, Marston G, Nutt DR, Shine KP, et al. Infrared absorption spectra, radiative efficiencies, and global warming potentials of perfluorocarbons: comparison between experiment and theory. J Geophys Res Atmos 2010b;115:1-12. doi:10.1029/2010JD014771.

[544] Young CJ, Hurley MD, Wallington TJ, Mabury SA. Atmospheric chemistry of $\mathrm{CF}_{3} \mathrm{CF}_{2} \mathrm{H}$ and $\mathrm{CF}_{3} \mathrm{CF}_{2} \mathrm{CF}_{2} \mathrm{CF}_{2} \mathrm{H}$ : Kinetics and products of gas-phase reactions with $\mathrm{Cl}$ atoms and $\mathrm{OH}$ radicals, infrared spectra, and formation of perfluorocarboxylic acids. Chem Phys Lett 2009b;473:251-6. doi:10.1016/j.cplett.2009. 04.001 .

[545] Gohar LK, Myhre G, Shine KP. Updated radiative forcing estimates of four halocarbons. J Geophys Res 2004;109:D01107. doi:10.1029/ 2003JD004320.

[546] Hurley MD, Wallington T], Buchanan GA, Gohar LK, Marston G, Shine KP. IR spectrum and radiative forcing of $\mathrm{CF}_{4}$ revisited. J Geophys Res D Atmos 2005;110:1-8. doi:10.1029/2004JD005201.

[547] Nielsen OJ, Nicolaisen FM, Bacher C, Hurley MD, Wallington TJ, Shine KP. Infrared spectrum and global warming potential of $\mathrm{SF}_{5} \mathrm{CF}_{3}$. Atmos Environ 2002;36 1237-40. doi:10.1016/S1352-2310(01)00551-9.

[548] Sung K, Toon GC, Mantz AW, Smith MAH. FT-IR measurements of cold $\mathrm{C}_{3} \mathrm{H}_{8}$ cross sections at 7-15 $\mu \mathrm{m}$ for Titan atmosphere. Icarus 2013;226:1499-513. doi:10.1016/j.icarus.2013.07.028.

[549] Buzan EM, Hargreaves RJ, Bernath PF. High resolution absorption cross sections for propylene in the $3 \mu \mathrm{m}$ region at high temperatures. Mol Astrophys 2016;3-4:16-20. doi:10.1016/j.molap.2016.06.001.

[550] Hargreaves RJ, Buzan E, Dulick M, Bernath PF. High-resolution absorption cross sections of $\mathrm{C}_{2} \mathrm{H}_{6}$ at elevated temperatures. Mol Astrophys 2015;1:205. doi:10.1016/j.molap.2015.09.001.

[551] Sung K, Toon GC, Crawford TJ. $\mathrm{N}_{2}$ - and $\left(\mathrm{H}_{2}+\mathrm{He}\right)$-broadened cross sections of benzene $\left(\mathrm{C}_{6} \mathrm{H}_{6}\right)$ in the $7-15 \mu \mathrm{m}$ region for the Titan and jovian atmospheres. Icarus 2016;271:438-52. doi:10.1016/j.icarus.2016.01.012.

[552] Beale CA, Hargreaves RJ, Bernath PF. Temperature-dependent high resolution absorption cross sections of propane. J Quant Spectrosc Radiat Transf 2016;182:219-24. doi:10.1016/j.jqsrt.2016.06.006.

[553] Andersen MPS, Nilsson EJK, Nielsen OJ, Johnson MS, Hurley MD, Wallington TJ. Atmospheric chemistry of trans- $\mathrm{CF}_{3} \mathrm{CHCHCl}$ : Kinetics of the gas-phase reactions with $\mathrm{Cl}$ atoms, $\mathrm{OH}$ radicals, and 03. J Photochem Photobiol A Chem 2008;199:92-7. doi:10.1016/j.jphotochem.2008.05.013.

[554] Hurley MD, Ball JC, Wallington TJ. Atmospheric chemistry of the Z and E isomers of $\mathrm{CF}_{3} \mathrm{CFCHF}$; kinetics, mechanisms, and products of gas-phase reactions with $\mathrm{Cl}$ atoms, $\mathrm{OH}$ radicals, and $\mathrm{O}_{3}$. J Phys Chem A 2007;111:9789-95. doi:10.1021/jp0753530

[555] Sulbaek Andersen MP, Waterland RL, Sander SP, Nielsen OJ, Wallington TJ. Atmospheric chemistry of $\mathrm{CxF}_{2} \mathrm{x}+1 \mathrm{CHCH}_{2} \quad(\mathrm{x}=1,2,4,6$ and 8): radiative efficiencies and global warming potentials. J Photochem Photobiol A Chem 2012b;233:50-2. doi:10.1016/j.jphotochem.2012.02.020.

[556] Nilsson EJK, Nielsen OJ, Johnson MS, Hurley MD, Wallington TJ. Atmospheric chemistry of cis- $\mathrm{CF}_{3} \mathrm{CH}$ \{double bond, long $\mathrm{CHF}$ : Kinetics of reactions with $\mathrm{OH}$ radicals and $\mathrm{O}_{3}$ and products of $\mathrm{OH}$ radical initiated oxidation. Chem Phys Lett 2009;473:233-7. doi:10.1016/j.cplett.2009.03.076.

[557] Sulbaek Andersen MP, Hurley MD, Wallington TJ, Blandini F, Jensen NR, Librando $\mathrm{V}$, et al. Atmospheric chemistry of $\mathrm{CH}_{3} \mathrm{O}\left(\mathrm{CF}_{2} \mathrm{CF}_{2} \mathrm{O}\right) \mathrm{nCH}_{3}(\mathrm{n}=1-3)$ : Kinetics and mechanism of oxidation initiated by $\mathrm{Cl}$ atoms and $\mathrm{OH}$ radicals, IR spectra, and global warming potentials. J Phys Chem A 2004;108:1964-72. doi:10.1021/jp036615a.

[558] Oyaro N, Sellevåg SR, Nielsen CJ. Study of the $\mathrm{OH}$ and $\mathrm{Cl}$-initiated oxidation, IR absorption cross-section, radiative forcing, and global warming potential of four C 4 -hydrofluoroethers. Environ Sci Technol 2004;38:5567-76. doi:10. 1021/es0497330.

[559] Oyaro N, Sellevåg SR, Nielsen CJ. Atmospheric chemistry of hydrofluoroethers: reaction of a series of hydrofluoroethers with $\mathrm{OH}$ radicals and $\mathrm{Cl}$ atoms, atmospheric lifetimes, and global warming potentials. J Phys Chem A 2005;109:337-46. doi:10.1021/jp047860c.

[560] Waterland RL, Hurley MD, Misner Ja, Wallington TJ, Melo SML, Strong K, et al. Gas phase UV and IR absorption spectra of $\mathrm{CF}_{3} \mathrm{CH}_{2} \mathrm{CH}_{2} \mathrm{OH}$ and $\mathrm{F}\left(\mathrm{CF}_{2} \mathrm{CF}_{2}\right) \mathrm{xCH}_{2} \mathrm{CH}_{2} \mathrm{OH}(\mathrm{x}=2,3$, 4). J Fluor Chem 2005;126:1288-96. doi:10. 1016/j.jfluchem.2005.06.010

[561] Sellevag SR, Kelly T, Sidebottom H, Nielsen CJ. A study of the IR and UV-Vis absorption cross-sections, photolysis and $\mathrm{OH}$-initiated oxidation of $\mathrm{CF}_{3} \mathrm{CHO}$ and $\mathrm{CF}_{3} \mathrm{CH}_{2} \mathrm{CHO}$. Phys Chem Chem Phys 2004a;6:1243-52. doi:10. 1039/b315941h.

[562] Hashikawa Y, Kawasaki M, Waterland RL, Hurley MD, Ball JC, Wallington TJ, et al. Gas phase UV and IR absorption spectra of $\mathrm{CxF}_{2} \mathrm{x}+1 \mathrm{CHO}(\mathrm{x}=1-4)$. Fluor Chem 2004;125:1925-32. doi:10.1016/j.jfluchem.2004.07.006.

[563] Sellevåg SR, Nielsen CJ, Søvde OA, Myhre G, Sundet JK, Stordal F, et al. Atmospheric gas-phase degradation and global warming potentials of 2fluoroethanol, 2,2-difluoroethanol, and 2,2,2-trifluoroethanol. Atmos Environ 2004b;38:6725-35. doi:10.1016/j.atmosenv.2004.09.023.

[564] Bravo I, Díaz-de-Mera Y, Aranda A, Smith K, Shine KP, Marston C. Atmospheric chemistry of $\mathrm{C}_{4} \mathrm{~F}_{9} \mathrm{OC}_{2} \mathrm{H}_{5}$ (HFE-7200), $\mathrm{C}_{4} \mathrm{~F}_{9} \mathrm{OCH}_{3}$ (HFE-7100), $\mathrm{C}_{3} \mathrm{~F}_{7} \mathrm{OCH}_{3}$ (HFE-7000) and $\mathrm{C}_{3} \mathrm{~F}_{7} \mathrm{CH}_{2} \mathrm{OH}$ : temperature dependence of the kinetics of their reactions with $\mathrm{OH}$ radicals, atmospheric lifetimes and global warming potentials. Phys Chem Chem Phys 2010a;12:5115-25. doi:10.1039| b923092k.

[565] Wallington TJ, Schneider WF, Barnes I, Becker KH, Sehested J, Nielsen OJ. Stability and infrared spectra of mono-, di-, and trichloromethanol. Chem Phys Lett 2000;322:97-102. doi:10.1016/S0009-2614(00)00384-5.

[566] Shine KP, Gohar LK, Hurley MD, Marston G, Martin D, Simmonds PG, et al Perfluorodecalin: global warming potential and first detection in the atmosphere. Atmos Environ 2005:39:1759-63. doi:10.1016/j.atmosenv.2005.01.001.

[567] Andersen MPS, Nielsen OJ, Wallington TJ, Karpichev B, Sander SP. Assessing the impact on global climate from general anesthetic gases. Anesth Analg 2012a;114:1081-5. doi:10.1213/ANE.0b013e31824d6150.

[568] Sulbaek Andersen MP, Sander SP, Nielsen OJ, Wagner DS, Sanford TJ, Wallington TJ. Inhalation anaesthetics and climate change. $\mathrm{Br} \mathrm{J}$ Anaesth 2010;105:760-6. doi:10.1093/bja/aeq259.

[569] Ryan SM, Nielsen CJ. Global warming potential of inhaled anesthetics: application to clinical use. Anesth Analg 2010;111:92-8. doi:10.1213/ANE. Ob013e3181e058d7

[570] Acerboni G, Beukes JA, Jensen NR, Hjorth J, Myhre G, Nielsen CJ, et al. Atmospheric degradation and global warming potentials of three perfluoroalkenes. Atmos Environ 2001;35:4113-23. doi:10.1016/S1352-2310(01)00209-6.

[571] Nielsen OJ, Javadi MS, Sulbaek Andersen MP, Hurley MD, Wallington TJ Singh R. Atmospheric chemistry of $\mathrm{CF}_{3} \mathrm{CFCH}_{2}$ : Kinetics and mechanisms of gas-phase reactions with $\mathrm{Cl}$ atoms, $\mathrm{OH}$ radicals, and $\mathrm{O}_{3}$. Chem Phys Lett 2007;439:18-22. doi:10.1016/j.cplett.2007.03.053. 
[572] Wallington TJ, Hurley MD, Ball JC, Ellermann T, Nielsen OJ, Sehested J. Atmospheric chemistry of HFC-152: UV absorption spectrum of $\mathrm{CH}_{2} \mathrm{FCFHO}_{2}$ radicals, kinetics of the reaction $\mathrm{CH}_{2} \mathrm{FCFHO}_{2}+\mathrm{NO}$.fwdarw. $\mathrm{CH}_{2} \mathrm{FCHFO}+\mathrm{NO}_{2}$, and fate of the alkoxy radical $\mathrm{CH}_{2}$ FCFHO. J Phys Chem 1994;98:5435-40. doi: $10.1021 / \mathrm{j} 100072 \mathrm{a} 008$

[573] Inoue $\mathrm{Y}$, Kawasaki $\mathrm{M}$, Wallington TJ, Hurley MD. Atmospheric chemistry of $\mathrm{CF}_{3} \mathrm{CH}_{2} \mathrm{CF}_{2} \mathrm{CH}_{3}$ (HFC-365mfc): kinetics and mechanism of chlorine atom initiated oxidation, infrared spectrum, and global warming potential. Chem Phys Lett 2008;462:164-8. doi:10.1016/j.cplett.2008.07.054.

[574] Mashino M, Ninomiya Y, Kawasaki M, Wallington TJ, Hurley MD. Atmospheric chemistry of $\mathrm{CF}_{3} \mathrm{CF}=\mathrm{CF}_{2}$ : kinetics and mechanism of its reactions with $\mathrm{OH}$ radicals, $\mathrm{Cl}$ atoms, and ozone. J Phys Chem A 2000;104:7255-60. doi:10.1021/ jp000498r.

[575] Østerstrøm FF, Nielsen OJ, Sulbaek Andersen MP, Wallington TJ. Atmospheric chemistry of $\mathrm{CF}_{3} \mathrm{CH}_{2} \mathrm{OCH}_{3}$ : reaction with chlorine atoms and $\mathrm{OH}$ radicals, kinetics, degradation mechanism and global warming potential. Chem Phys Lett 2012;524:32-7. doi:10.1016/j.cplett.2011.12.047.

[576] Wallington TJ, Hurley MD, Nielsen OJ, Sulbaek Andersen MP. Atmospheric chemistry of $\mathrm{CF}_{3} \mathrm{CFHCF}_{2} \mathrm{OCF}_{3}$ and $\mathrm{CF}_{3} \mathrm{CFHCF}_{2} \mathrm{OCF}_{2} \mathrm{H}$ : reaction with $\mathrm{Cl}$ atoms and $\mathrm{OH}$ radicals, degradation mechanism, and global warming potentials. $\mathrm{J}$ Phys Chem A 2004;108:11333-8. doi:10.1021/jp046454q.

[577] Søndergaard R, Nielsen OJ, Hurley MD, Wallington TJ, Singh R. Atmospheric chemistry of trans- $\mathrm{CF}_{3} \mathrm{CHCHF}$ : kinetics of the gas-phase reactions with $\mathrm{Cl}$ atoms, $\mathrm{OH}$ radicals, and $\mathrm{O}_{3}$. Chem Phys Lett 2007;443:199-204. doi:10.1016/ j.cplett.2007.06.084.

[578] Myhre G, Nielsen C, Powell D, Stordal F. Infrared absorption cross section, radiative forcing, and GWP of four hydrofluoro(poly)ethers. Atmos Environ 1999;33:4447-58. doi:10.1016/S1352-2310(99)00208-3.

[579] Wallington TJ, Hurley $\mathrm{MD}$, Nielsen OJ. The radiative efficiency of $\mathrm{HCF}_{2} \mathrm{OCF}_{2} \mathrm{OCF}_{2} \mathrm{CF}_{2} \mathrm{OCF}_{2} \mathrm{H}$ (H-Galden 1040x) revisited. Atmos Environ 2009;43:4247-9. doi:10.1016/j.atmosenv.2009.05.046.

[580] Ninomiya Y, Kawasaki M, Guschin A, Molina LT, Molina MJ, Wallington TJ Atmospheric chemistry of $\mathrm{n}-\mathrm{C}_{3} \mathrm{~F}_{7} \mathrm{OCH}_{3}$ : reaction with $\mathrm{OH}$ radicals and $\mathrm{Cl}$ atoms and atmospheric fate of $\mathrm{n}^{-} \mathrm{C}_{3} \mathrm{~F}_{7} \mathrm{OCH}_{2} \mathrm{O}(\bullet)$ radicals. Environ Sci Technol 2000;34:2973-8. doi:10.1021/es991449z.

[581] Robson JI, Gohar LK, Hurley MD, Shine KP, Wallington TJ. Revised IR spectrum, radiative efficiency and global warming potential of nitrogen trifluoride. Geophys Res Lett 2006;33:2-4. doi:10.1029/2006GL026210.

[582] D’Anna B, Sellevåg SR, Wirtz K, Nielsen CJ. Photolysis study of perfluoro-2methyl-3-pentanone under natural sunlight conditions. Environ Sci Technol 2005;39:8708-11. doi:10.1021/es048088u.

[583] Young CJ, Hurley MD, Wallington TJ, Mabury SA. Atmospheric chemistry of perfluorobutenes $\left(\mathrm{CF}_{3} \mathrm{CFCFCF}_{3}\right.$ and $\left.\mathrm{CF}_{3} \mathrm{CF}_{2} \mathrm{CFCF}_{2}\right)$ : Kinetics and mechanisms of reactions with $\mathrm{OH}$ radicals and chlorine atoms, IR spectra, global warming potentials, and oxidation to perfluorocarboxylic acids. Atmos Environ 2009a;43:3717-24. doi:10.1016/j.atmosenv.2009.04.025

[584] Young CJ, Hurley MD, Wallington TJ, Mabury SA. Atmospheric lifetime and global warming potential of a perfluoropolyether. Environ Sci Technol 2006:40:2242-6. doi:10.1021/es052077z.

[585] Sulbaek Andersen MP, Blake DR, Rowland FS, Hurley MD, Wallington TJ. Atmospheric chemistry of sulfuryl fluoride: reaction with $\mathrm{OH}$ radicals, $\mathrm{Cl}$ atoms and $\mathrm{O}_{3}$, atmospheric lifetime, IR spectrum, and global warming potential. Environ Sci Technol 2009;43:1067-70. doi:10.1021/es802439f.

[586] Richard C, Gordon IE, Rothman LS, Abel M, Frommhold L, Gustafsson M, et al. New section of the HITRAN database: collision-induced absorption (CIA). J Quant Spectrosc Radiat Transf 2012;113:1276-85. doi:10.1016/j.jqsrt.2011.11. 004.

[587] Maté B, Lugez C, Fraser GT, Lafferty WJ. Absolute intensities for the $\mathrm{O}_{2}$ $1.27 \mu \mathrm{m}$ continuum absorption. J Geophys Res Atmos 1999;104:30585-90. doi:10.1029/1999JD900824

[588] Smith KM, Newnham DA. Near-infrared absorption cross sections and integrated absorption intensities of molecular oxygen $\left(\mathrm{O}_{2}, \mathrm{O}_{2}-\mathrm{O}_{2}\right.$, and $\left.\mathrm{O}_{2}-\mathrm{N}_{2}\right)$. J Geophys Res Atmos 2000;105:7383-96. doi:10.1029/1999JD901171.

[589] Eldering A, Boland S, Solish B, Crisp D, Kahn P, Gunson M. High precision atmospheric $\mathrm{CO}_{2}$ measurements from space: The design and implementation of OCO-2. In: 2012 IEEE Aerosp. Conf.. IEEE; 2012. p. 1-10. doi:10.1109/AERO. 2012.6187176

[590] Thomas GE, Stamnes K. Radiative transfer in the atmosphere and ocean. Cambridge: Cambridge University Press; 1999.

[591] Brasseur GP, Solomon S. Aeronomy of the middle atmosphere. Dordrecht: Springer; 2005

[592] Downing HD, Williams D. Optical constants of water in the infrared. J Geophys Res 1975;80:1656-61. doi:10.1029/JC080i012p01656

[593] Kou L, Labrie D, Chylek P. Refractive indices of water and ice in the 0.65- to 2.5- $\mathrm{mm}$ spectral range. Appl Opt 1993;32:3531. doi:10.1364/AO.32. 003531.

[594] Warren SG, Brandt RE. Optical constants of ice from the ultraviolet to the microwave: a revised compilation. J Geophys Res 2008;113:D14220. doi:10. 1029/2007JD009744

[595] Fenn RW, Clough SA, Gallery WO, Good RE, Kneizys FX, Mill JD, et al.Jursa AS, editor Optical and infrared properties of the atmosphere. Handb. Geophys. Sp. Environ. 1985

[596] Palmer KF, Williams D. Optical constants of sulfuric acid; application to the clouds of Venus? Appl Opt 1975;14:208. doi:10.1364/AO.14.000208.

[597] Remsberg EE, Lavery D, Crawford B. Optical constants for sulfuric and nitric acids. J Chem Eng Data 1974;19:263-5. doi:10.1021/je60062a003.
[598] Tisdale RT, Glandorf DL, Tolbert MA, Toon OB. Infrared optical constants of low-temperature $\mathrm{H}_{2} \mathrm{SO}_{4}$ solutions representative of stratospheric sulfate aerosols. J Geophys Res Atmos 1998;103:25353-70. doi:10.1029/98JD02457.

[599] Niedziela RF, Norman ML, DeForest CL, Miller RE, Worsnop DR. A temperature- and composition-dependent study of $\mathrm{H}_{2} \mathrm{SO}_{4}$ aerosol optical constants using Fourier transform and tunable diode laser infrared spectroscopy. J Phys Chem A 1999;103:8030-40. doi:10.1021/jp991323o.

[600] Biermann UM, Luo BP, Peter T. Absorption spectra and optical constants of binary and ternary solutions of $\mathrm{H}_{2} \mathrm{SO}_{4}, \mathrm{HNO}_{3}$, and $\mathrm{H}_{2} \mathrm{O}$ in the mid infrared at atmospheric temperatures. J Phys Chem A 2000;104:783-93. doi:10.1021/ jp992349i.

[601] Querry MR, Tyler IL. Reflectance and complex refractive indices in the infrared for aqueous solutions of nitric acid. J Chem Phys 1980;72:2495-9. doi:10.1063/1.439445.

[602] Norman ML, Qian J, Miller RE, Worsnop DR. Infrared complex refractive indices of supercooled liquid $\mathrm{HNO}_{3} / \mathrm{H}_{2} \mathrm{O}$ aerosols. J Geophys Res Atmos 1999;104:30571-84. doi:10.1029/1999JD900902.

[603] Toon OB, Tolbert MA, Koehler BG, Middlebrook AM, Jordan J. Infrared optical constants of $\mathrm{H}_{2} \mathrm{O}$ ice, amorphous nitric acid solutions, and nitric acid hydrates. J Geophys Res 1994;99:25631. doi:10.1029/94JD02388.

[604] Niedziela RF, Miller RE, Worsnop DR. Temperature- and frequency-dependent optical constants for nitric acid dihydrate from aerosol spectroscopy. J Phys Chem A 1998;102:6477-84. doi:10.1021/jp981299z.

[605] Richwine LJ, Clapp ML, Miller RE, Worsnop DR. Complex refractive indices in the infrared of nitric acid trihydrate aerosols. Geophys Res Lett 1995;22:2625-8. doi:10.1029/95GL02650.

[606] Sutherland RA, Khanna RK. Optical properties of organic-based aerosols produced by burning vegetation. Aerosol Sci Technol 1991;14:331-42. doi:10. 1080/02786829108959495.

[607] Magi BI, Fu Q, Redemann J. A methodology to retrieve self-consistent aerosol optical properties using common aircraft measurements. J Geophys Res 2007 112:D24S12. doi:10.1029/2006JD008312.

[608] Stagg BJ, Charalampopoulos TT. Refractive indices of pyrolytic graphite, amorphous carbon, and flame soot in the temperature range $25^{\circ}$ to $600{ }^{\circ} \mathrm{C}$. Combust Flame 1993;94:381-96. doi:10.1016/0010-2180(93)90121-I.

[609] Chang H, Charalampopoulos TT. Determination of the wavelength dependence of refractive indices of flame soot. Proc R Soc A Math Phys Eng Sci 1990;430:577-91. doi:10.1098/rspa.1990.0107.

[610] Querry MR. Optical constants of minerals and other materials from the millimeter to the ultraviolet. Aberdeen: Chemical Research, Development Engineering Center, CRDEC-CR-88009:; 1987.

[611] Alexander DTL, Crozier PA, Anderson JR. Brown carbon spheres in East Asian outflow and their optical properties. Science (80-) 2008;321:833-6. doi:10. 1126/science.1155296.

[612] Lund Myhre CE, Nielsen CJ. Optical properties in the UV and visible spectral region of organic acids relevant to tropospheric aerosols. Atmos Chem Phys 2004;4:1759-69. doi:10.5194/acp-4-1759-2004.

[613] Hasenkopf CA, Beaver MR, Trainer MG, Langley Dewitt $H$, Freedman MA Toon OB, et al. Optical properties of Titan and early Earth haze laboratory analogs in the mid-visible. Icarus 2010;207:903-13. doi:10.1016/j.icarus.2009. 12.015 .

[614] Zarzana KJ, De Haan DO, Freedman MA, Hasenkopf CA, Tolbert MA. Optical properties of the products of $\alpha$-dicarbonyl and amine reactions in simulated cloud droplets. Environ Sci Technol 2012;46:4845-51. doi:10.1021/es2040152.

[615] Toon OB, B Pollack J, Sagan C. Physical properties of the particles composing the Martian dust storm of 1971-1972. Icarus 1977;30:663-96. doi:10.1016/ 0019-1035(77)90088-4

[616] Wagner R, Ajtai T, Kandler K, Lieke K, Linke C, Müller T, et al. Complex refractive indices of Saharan dust samples at visible and near UV wavelengths: a laboratory study. Atmos Chem Phys 2012;12:2491-512. doi:10. 5194/acp-12-2491-2012.

[617] Sinyuk A, Torres O, Dubovik O. Combined use of satellite and surface observations to infer the imaginary part of refractive index of Saharan dust. Geophys Res Lett 2003;30. doi:10.1029/2002GL016189.

[618] Grainger RG, Peters DM, Thomas GE, Smith AJA, Siddans R, Carboni E, et al. Measuring volcanic plume and ash properties from space Remote sensing of volcanoes and volcanic Processes: integrating observation and modeling. Pyle D, Mather T, editors. Spec Publ Geo Soc; 2013. doi:10.1144/SP380.14.

[619] Henning T, Mutschke $\mathrm{H}$. Low temperature infrared properties of cosmic dust analogues. Astron Astrophys 1997;327:743-54 http://aa.springer.de/papers/ 7327002/2300743/small.htm.

[620] Zeidler S, Posch T, Mutschke H. Optical constants of refractory oxides at high temperatures. Astron Astrophys 2013;553:A81. doi:10.1051/0004-6361/ 201220459.

[621] Begemann B, Dorschner J, Henning T, Mutschke H, Gurtler J, Kompe C, et al. Aluminum Oxide and the Opacity of Oxygen-rich Circumstellar Dust in the 12-17 $\mu$ range. Astrophys J 1997:476:199-208, doi:10.1086/303597.

[622] Henning T, Begemann B, Mutschke H, Dorschner J. Optical properties of oxide dust grains. Astron Astrophys Suppl Ser 1995;112:143-9.

[623] Posch T, Kerschbaum F, Fabian D, Mutschke H, Dorschner J, Tamanai A, et al. Infrared properties of solid titanium oxides: exploring potential primary dust condensates. Astrophys J Suppl Ser 2003;149:437-45. doi:10.1086/379167.

[624] Unpublished data n.d. http://www.astro.uni-jena.de/Laboratory/OCDB/ mgfeoxides.html\#C.

[625] Unpublished data n.d. http://www.astro.uni-jena.de/Laboratory/OCDB/ crsilicates.html\#C. 
ID: JQSRT

[m5G ;August 21, 2017;14:18]

66

I.E. Gordon et al./Journal of Quantitative Spectroscopy \& Radiative Transfer 000 (2017) 1-66

[626] Fabian D, Kenning T, Jäger C, Mutschke H, Dorschner J, Wehrhan O. Steps toward interstellar silicate mineralogy. Astron Astrophys 2001;378:228-38. doi:10.1051/0004-6361:20011196.

[627] Fabian D, Posch T, Mutschke H, Kerschbaum F, Dorschner J. Infrared optical properties of spinels, A study of the carrier of the 13,17 and $32 \mu$ emission features observed in ISO-SWS spectra of oxygen-rich AGB stars. Astron Astrophys 2001;373:1125-38. doi:10.1051/0004-6361:20010657.

[628] Jäger C, Dorschner J, Mutschke H, Posch T, Henning T. Steps toward interstellar silicate mineralogy VII. Spectral properties and crystallization behaviour of magnesium silicates produced by the sol-gel method. Astron Astrophys 2003;408:193-204. doi:10.1051/0004-6361:20030916.

[629] Zeidler S, Pooch T, Mutschke H, Richter H, Wehrhan O. Near-infrared absorption properties of oxygen-rich stardust analogs: The influence of colring metal ions. Astron Astrophys 2011;526:A68. doi:10.1051/0004-6361| 201015219.

[630] Posch T, Kerschbaum F, Mutschke HD, Fabian D, Clément D, Dorschner J. Fatyres of oxide dust particles in circumstellar shells of AGB stars. In: Exploit. ISO Data Arch. Infrared Astron. Internet Age, Siguenza, Spain, June 24-27; 2002. p. 14.

[631] Khare BN, Sagan C, Arakawa ET, Suits F, Callcott TA, Williams MW. Optical constants of organic tholins produced in a simulated Titania atmosphere: From soft x-ray to microwave frequencies. Icarus 1984;60:127-37. doi:10.1016/0019-1035(84)90142-8.

[632] Ramirez S, Coll IP, da Silva A, Navarro-Gonzalez R, Lafait J, Raulin F. Comflex refractive index of Titan's aerosol analogues in the $200-900 \mathrm{~nm}$ Domain. Icarus 2002;156:515-29. doi:10.1006/icar.2001.6783.

[633] Bohren CF, Huffman DR. Absorption and scattering of light by small particles. New York: John Wiley and Sons; 1983. doi:10.1002/9783527618156.
[634] Wakeford HR, Sing DK. Transmission spectral properties of clouds for hot Jupiter exoplanets. Astron Astrophys 2015;573:A122. doi:10.1051/0004-6361/ 201424207.

[635] Massie ST, Hervig M. HITRAN 2012 refractive indices. J Quant Spectrosc Radiant Transf 2013;130:373-80. doi:10.1016/j.jqsrt.2013.06.022.

[636] Kochanov R V, Gordon IE, Rothman LS, Sharpe SW, Johnson TJ, Sam RL. Comment on "Radiative forcing for 28 potential Archean greenhouse gases" by Byrne and Goldblatt (2014. Crim Past 2015;11:1097-105. doi:10.5194/ cp-11-1097-2015.

[637] Fischer J, Gamache RR, Goldman a, Rothman LS, Perrin a. Total internal partiion sums for molecular species in the 2000 edition of the HITRAN database. J Quant Spectrosc Radial Transf 2003;82:401-12. doi:10.1016/S0022-4073(03) 00166-3.

[638] Fischer J, Gamache RR. Total internal partition sums for molecules of astrophysical interest. J Quant Spectrosc Radial Transf 2002;74:263-72. doi:10. 1016/S0022-4073(01)00234-5.

[639] Goldman A, Gamache RR, Perrin A, Fraud J-M, Rinsland CP, Rothman LS. HITRAN partition functions and weighted transition-moments squared. J Quant Spectrosc Radial Transf 2000;66:455-86. doi:10.1016/S0022-4073(99) 00176-4.

[640] Herzberg G. Infrared and ramen spectra of polyatomic molecules. New Jersey: D. Van Nostrand Company, Inc.; 1960.

[641] Gamache RR, Roller C, Lopes E, Gordon IE, Rothman LS, Polyansky OL, et al. Total internal partition sums for 166 isotopologues of 51 molecules important in planetary atmospheres: application to HITRAN2016 and beyond. J Quant Spectrosc Radial Transf 2017 this issue. doi:10.1016/j.jqsrt.2017.03.045.

Please cite this article as: I.E. Gordon et al., The HITRAN2016 molecular spectroscopic database, Journal of Quantitative Spectroscopy \&

Radiative Transfer (2017), http://dx.doi.org/10.1016/j.jqsrt.2017.06.038 